UC-62

\title{
1980 Annual Report of the Coolidge Solar Irrigation Project
}

Leroy Torkelson, Sandia National Laboratories

Dennis L. Larson, The University of Arizona

Prepared by Sandia National Laboratories, Albuquerque, New Mexico 87185

and Livermore, California 94550 for the United States Department

of Energy under Contract DE-ACO4-76DPO0789

Printed February 1981

\section{[i] Sandia National Laboratories}




\section{DISCLAIMER}

This report was prepared as an account of work sponsored by an agency of the United States Government. Neither the United States Government nor any agency Thereof, nor any of their employees, makes any warranty, express or implied, or assumes any legal liability or responsibility for the accuracy, completeness, or usefulness of any information, apparatus, product, or process disclosed, or represents that its use would not infringe privately owned rights. Reference herein to any specific commercial product, process, or service by trade name, trademark, manufacturer, or otherwise does not necessarily constitute or imply its endorsement, recommendation, or favoring by the United States Government or any agency thereof. The views and opinions of authors expressed herein do not necessarily state or reflect those of the United States Government or any agency thereof. 


\section{DISCLAIMER}

Portions of this document may be illegible in electronic image products. Images are produced from the best available original document. 
Issued by Sandia National Laboratories, operated for the United States Department of Energy by Sandia Corporation.

NOTICE: This report was prepared as an account of work sponsored by an agency of the United States Government. Neither the United States Government nor any agency thereof, nor any of their employees, nor any of their contractors, subcontractors, or their employees, makes any warranty, express or implied, or assumes any legal liability or responsibility fro the accuracy, completeness, or use fuiness of any information, apparatus, product, or process disclosed, or represents that its use would not infringe privately uw ned rights. Refercnce herein to any specific commercial product, prness, or service imply its endorsement, recommendation, or favoring by the United States Government, any agency thereof or any of their contractors or subcontractors. The views and opinions expressed herein do not necessarily state or reflect those of the United States Government, any agency thereof or any of their contractors or subcontractors.

Printed in the United States of America

Available from

National Technical Information Service

U. S. Department of Commerce

5285 Port Royal Road

Springfield, VA 22161

Price: Printed Copy $\$ 11.00$; Microfiche: A0l 


\section{PAGES 1 to 2 WERE INTENTIONALLY LEFT BLANK}


SAND80-2378

Unlimited Release

Printed February 1981

1980

ANNUAL REPORT

OF THE

COOLIDGE SOLAR, IRRIGATION PROJECT

Leroy Torkelson

9511100

Sandia National Laboratories

Experimental Systems Operations Division

Albuquerque, New Mexico 87185

and

- Dennis L. Larson

$950^{4881}$

The University of Arizona.

Soils, Water and Engineering Department

Tucson, Arizona 85721

\begin{abstract}
The Coolidge Solar Irrigation Facility at Coolidge, Arizona, consists of a 2136.8-m2 $\left(23,000-\mathrm{ft}^{2}\right)$ linefocus collector subsystem, a $113.55-\mathrm{m}^{3}$ (30,000-gallon) thermal storage subsystem, and a $150-\mathrm{kWe}(142.2-\mathrm{Btu} / \mathrm{s})$ power generation unit. The purpose of this document is to report the performance of the facility and its operational and maintenance requirements. This document covers the period of time from the facility's initial operation in October 1979 to 31 August 1980 .
\end{abstract}

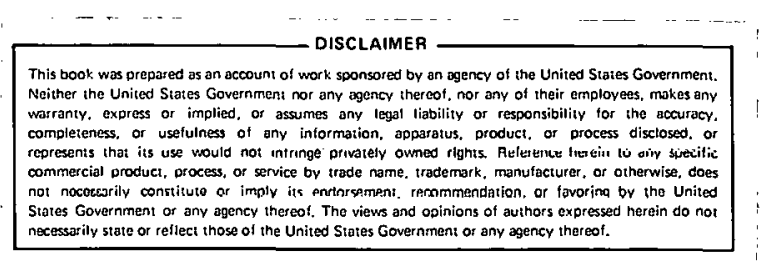




\section{ACKNOWLEDGMENT}

The authors acknowledge the following contributions:

The section "Solar Collector Subsystem Performance Predictions" was contributed by L. L. Lukens.

The scction "Examples of Thermosiphoning at Llie Cülidye, Arizona, Solar Irrigation Facility" was contributed by R. W. Harrigan.

The section "Construction Costs for the Coolidge, Arizona, Solar Irrigation Facility" was contributed by Marx Matteo, Acurex Corporation.

Jack Hoopes, Lee Ballard, and Ruben Wood were responsible for operating and maintaining the Coolidge Solar Irrigation Facility. The facility performance data were processed by Tim Clark of the University of Arizona. 
CONTENTS

Section

Page

INTRODUCTION

Figure 1. 150-kW Solar-Powered Irrigation Facility Flow Diagram

Table 1. Subsystem Description

OVERALL SUMMARY

Figure 1. Electrical Energy Generated by the Plant from January through August 1980

ENERGY COLLECTION AND PRODUCTION OF THE COOLIDGE, ARIZONA, SOLAR IRRIGATION FACILITY FOR THE PERIOD 1 JANUARY 1980 THROUGH 31 AUGUST 1980

Figure 1. Solar Energy Available and Thermal Energy Collected by the Plant. from January through August 1980

Figure 2. Electrical Energy Generated by the Plant from January through August 1980

PERFORMANCE OF THE SOLAR COLLECTOR SUBSYSTEM AT THE COOLIDGE SOLAR IRRIGATION FACILITY ON 17, 18, AND 24 DECEMBER 1979

Figure 1. Insolation, 351-1979

Figure 2. Flow Rate, 35l-1979

Figure 3. Inlet and Outlet Temperatures, 351-1979

Figure 4, Efficiency, 351-1979

Figure 5. Collected Power, 351-1979

Figure 6. Insolation, 352-1979

Figure 7. Flow Rate, 352-1979

Figure 8. Inlet and Outlet Temperatures, 352-1979 45

Figure 9. Efficiency, 352-1979 , * 46

Figure 10, Collected Power, 352-1979 47

Figure 11. Insolation, 358-1979 48

Figure 12. Flow Rate, 358-1979 49

Figure 13. Inlet and Outlet Temperatures, 358-1979 50

Figure 14.." Efficiency, 358-1979.

Figure 15. Collected Power, 358-1979 
SOLAR COLLECTOR SYSTEM VERNAL EQUINOX PERFORMANCE,

20 AND 23 MARCH AND 3. APRIL 1980

Table 1. Times Recorded on Days of Spring Equinox Collector Tests

Figure 1: Collected Power, 80-1980 56

Figure 2. Efficiency, 80-1980. 56

Figure 3. Insolation, 80-1980 57

Figure 4. Wind Speed and Ambient Temperature, 80-1980 57

Pigure j: Inlet and outlet Temperatures, 80-1980 . 58

Figure 6. Flow Rate, 80-1980 58

Figure 7. Collected power, 83-1980 60

Figure 8. Efficiency, 83-1980 60

Figure 9. Insolation, 83-1980 61

Figure 10. Wind Speed and Ambient Temperature, 83-1980.61

Figure 11. Inlet and outlet Temperatures, 83-1980 62

Figure 12. Flow Rate, 83-1980 62

Figure 13. Collected Power; 94-1980 64

Figure 14. Efficiency, 94-1980 64

Figure 15. Insolation, 94-1980 65

Figure 16. Wind Speed and Ambicnt Tempcraturc, 94-1980 65

Figure 17. Inlet and outlet Temperatures, 94-1980 66

Figure 18. Flow Rate, 94-1980 66

PERFORMANCE OF THE SUBSYSTEM AT COOLIDGE; ARIZONA, 25 JUNE AND 4 AND 5 JULY 1980

Table 1. Times Recorded for Performance Test Events 68

Figure 1. Collected Power, 177-1980 70

Figure 2. Efficiency, 177-1980 70

F'igure 3. Inlet and Outlet Temperatures, 177-1980

Figure 4. Flow Rate, 177-1980 71

Figure 5. Insolation, 177-1980 72

Figure 6. Wind Speed and Ambient Temperature, 177-1980 72

Figure 7. Collected Power, 186-1980 74

Figure 8. Efficiency, 186-1980 74

Figure 9. Inlet and outlet Temperatures, 186-1980 75 


\section{CONTENTS (Continued)}

$\underline{\text { Section }}$

Page

Figure 10. Flow Rate, 186-1980

Figure 11. Insolation, 186-1980

Figure 12. Wind Speed and Ambient Temperature, 186-1980

Figure 13. Collected Power, 187-1980

Figure 14. Efficiency, 187-1980

Figure 15. Inlet and Outlet Temperatures, 187-1980 80

Figure 16. Flow Rate, 187-1980 80

Figure 17. Insolation, 187-1980

Figure 18. Wind Speed and Ambient Temperature, 187-1980

Figure 1. Calculated Collector Field Subsystem Thermal output: Dirty Collectors

Figure 2. Calculated Collector Field Subsystem Efficiency: Dirty.Collectors

Figure 3. Calculated Collector Field Subsystem Thermal Output: Continuously Cleaned Collectors

Figure 4. Calculated Collector Field Subsystem Efficiency: Continuously Cleaned Collectors

STEADY-STATE THERMAL LOSS TEST OF THE COLLECTOR SUBSYSTEM AT THE COOLIDGE, ARIZONA, SOLAR IRRIGATION FACILITY, 15 APRIL 1980

Figure 1. Fluid Loop Schematic

Table 1. Collected Temperature Data, ${ }^{\circ} \mathrm{F}$

Figure 2. Thermal Losses vs. Midpoint Receiver Temperature above Ambient

SUNDSTRAND ORGANIC RANKINE CYCLE SUBSYSTEM PERFORMANCE TESTS AT THE COOLIDGE, ARIZONA, SOLAR IRRIGATION FACILITY, 29, 30, AND 31 JANUARY 1980

Figurs 1. Schematic Diagram of the 150-kW. Solar. Irrigation Facility

Figure 2. Vaporizer Assembly

Figure 3. Power Conversion Module

Table 1: Test Data

Figure 4. Generator Output, $\mathrm{kW}_{\mathrm{e}}$ 105

Table 2. Design Point Parameters 106

Table 3. Parasitic Power Consumption 107 
AN ESTIMATE OF THE PARASITIC ENERGY REQUIREMENT OF THE COOLIDGE SOLAR IRRIGATION FACILITY

STATIC TEST OF THERMOCLINE STORAGE TANK AT COOLIDGE; ARIZONA, 14 THROUGH 16 NOVEMBER 1979

Figure 1. Thermal Storage Tank Temperature Profile

Figure 2. Upper Bulk Caloria Temperature vs. Time

Figure 3. Extra Thermocouple Readings on Thermal Storage Tank

Figure 4. Ambient Air Temperature and Wind Velocity vs. 'l'ime

COOLIDGE, ARIZONA, THERMAL STORAGE SUBSYSTEM THERMOCLINE GROWTH TEST, 17 THROUGH 21 APRIL 1980

Figure 1. Tank Configuration

Figure 2. Thermocline Thickness vs. Time

Figure 3. Thermal Storage Tank Temperature Profile, 17 April 1980

Figure 4. Thermal Storage Tank Temperature. Profile, 18 April 1980

Figure 5. Thermal Storage Tank Temperature Profile, 19 Apri1 1980

Figure 6. Thermal Storage Tank Temperature Profile, 20 Äpri. 1980

Pigure 7. Thcrmal storage Tank Temperature Profile, 21 April 1980

Figure 1. Mixing. Tank and Thermocline. Tank Piping

EQUIPMENT PROBLEMS AND SOLUTIONS FOR THE COOLIDGE', ARIZONA, SOLAR IRRIGATION FACILITY

OPERATING COSTS FOR THE COOLIDGE, ARIZONA, SOLAR IRR'MÁTION FACILITY

RECURRING MAINTENANCE REQUIREMENTS FOR THE COOLIDGE, ARIZONA, SOIAR IRRIGATION FACILITY

Table 1 .

An Estimate of Recurring Maintenance Requirements for Collector, Fluid Transfer and Storage, and Power Conversion Systems

CONSTRUCTION COSTS FOR THE COOLIDGE, ARIZONA, SOLAR IRRIGATION FACILITY

Table 1. Breakdown of Construction Costs 


\section{INTRODUCTION}

This document is composed of a collection of reports written on the performance of the Coolidge, Arizona, Solar Irrigation Facility during its first year of operation.

The facility is the world's largest solar thermal power plant. The site, which is the Dalton Cole farm south of Coolidge; Arizona, was selected in February 1977. A preliminary design study of the facility was undertaken early in 1977 by three contractors and completed in August 1977. On the basis of the conceptual design competition, Acurex Corporation was selected as the prime contractor for this project as well as the supplier of the solar collectors. The major subcontractors to Acurex are Sundstrand Corporation and Sullivan and Masson Consulting Engineers. Sundstrand is the supplier of the Organic Rankine Cycle (ORC) power generation unit. The team of Sullivan and Masson and Acurex was responsible for the detailed design task.

The collector field is made up of $2140.49 \mathrm{~m}^{2}\left(23,040 \mathrm{ft}^{2}\right)$ of Acurex-supplied line-focusing parabolic trough collectors arranged in eight loops having a north-south orientation. The system is designed around three heat transfer loops. One loop extracts warm heattransfer oil from the bottom of a thermal storage tank, circulates the oil through the collector field, and returns it hot to the top of the thermal storage tank. The second loop extracts hot oil from the top of the storage tank, circulates the oil through a vaporizer heat exchange unit, and returns it to the bottom of the storage tank or directly to the collector field inlet. The third loop circulates liquid toluene through the vaporizer heat exchange unit to vaporize it and then expands the vapor through the turbine in the power conversion module to extract the energy for electrical power generation. The 
cycle is completed by condensing the expanded low-enthalpy vapor and pumping the condensate back to the vaporizer. The system flow diagram is shown in Figure 1.

The solar energy is converted to electrical energy by means of an ORC power conversion module, using toluene as the working fluid. The unit is complete with gear reduction and a 440-volt ac, 60-hertz, high-efficiency generator. Supporting equipment includes a vapor condenser for condensing the toluene and a vaporizer assembly consisting. of a preheater, an evaporator, and a superheater for vaporizing the toluene.

Enelgy ir ritored 1n a $113.55 \mathrm{~m}^{3}(30,000-g a l l o n)$ insulated tank 4.1666 metres ( 13.67 feet) in diameter and 14.9 metres (49 feet) $\mathrm{high}$. Varinus pumps, valves, and auxiliary tanks are included, and an underground tank is provided for the makeup heat transfer oil.

The control subsystem monitors and controls the collection and storage of solar energy and the generation and supply of electric power. In addition, the subsystem protects against system-related anomalies such as high temperatures in the collector field as weil as natural events such as high gusty winds.

The main control functions are

- Cullector tracking

- Field flow

- Collector loop flow

- Thermal storage

- ORC system

- Overtemperature protection

- High-wind protection

These control functions are largely independent, i.e., not cascaded, have built-in fail-safe action or directly acting limiting devices, and are based primarily on closed loop control and analog signal. transmission.

The data acquisition subsystem monitors the performance of the system and measures the auxiliary power consumed by the system. 


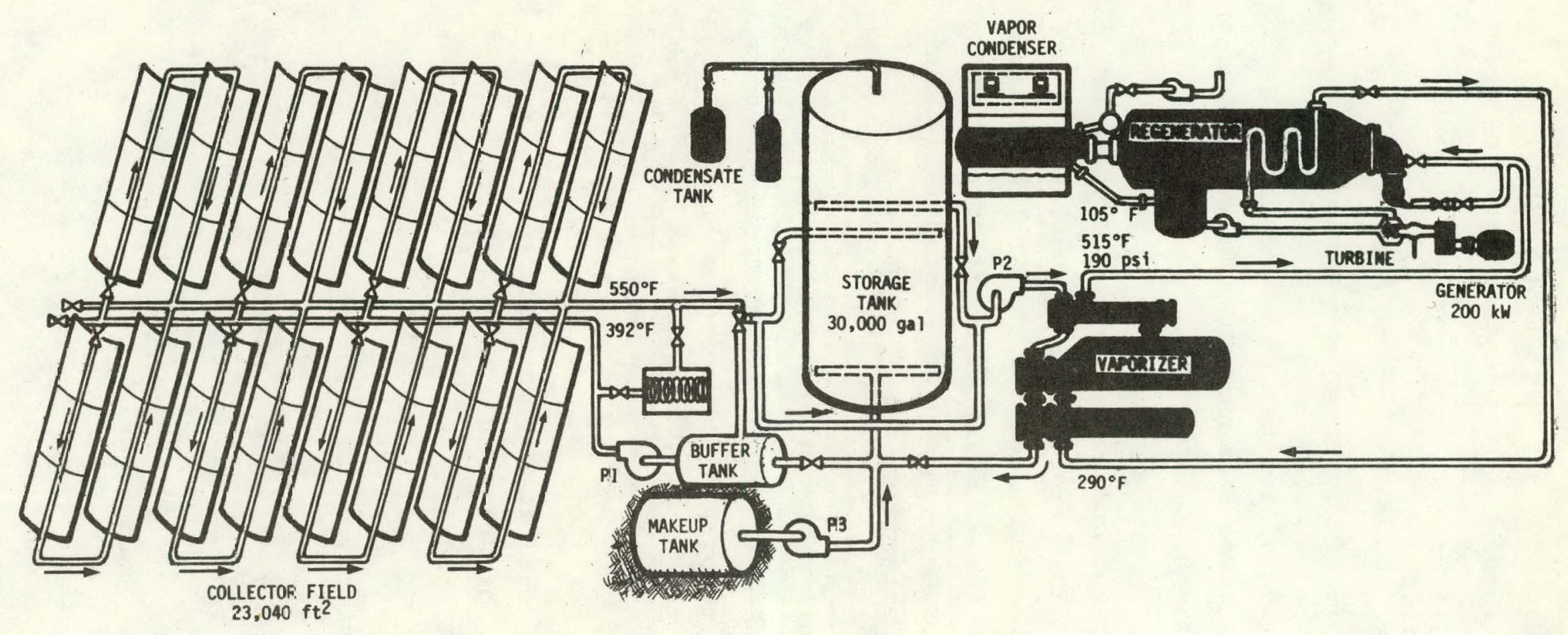

Figure 1. 150-kW Solar-Powered Irrigation Facility Flow Diagram 
The data acquired are used for plant control and for the performance analysis of main plant components. Data are derived from

- Weather conditions

- Collector fields

- Storage tank

- ORC unit

- Plant electrical output

Most of the data collected consist of conventional temperature, pressure, flow rate, and power measurements. A summary of the major system elements is given in Table 1.

Table 1 indicates a collector field subsystem efficiency of $38.6 \%$ at summer solstice. This tield of Acurex solar culleclors utilizes rnil zak for its reflective material. The reflectivity of the Coilzak has been found to be $60 \%$ as measured by a portable reflectometer. The performance of this subsystem can be substantially improved by the use of better reflector materials.

For a more complete description of the facility, refer to the following report:

D. Duffy, M. Matteo, and D. Ratinejad, Design, Construclion, and Operation of a $150 \mathrm{~kW}$ Solar-Powered Irrigation Facility,

ALO/4159-1.

Available from

National Technical Information Service

U.S. Department of Commerce

5285 Port Royal Road

Springfield, VA 22161

Price: printed copy $\$ 7.25$; microfiche $\$ 3.00$

During 1980, the facility was operated, tested, and evaluated in accordance with the following report:

L. E. Torkelson, $150 \mathrm{kWe}$ Solar Irrigation Project Test and

Evaluation Plan, SAND80-1568. 
Table 1

Subsystem Description

\section{Collector Field}

Size:

Fluid:

Temperatures:

Design conditions:
48 Acurex collector groups with N-S axis orientation $=23,040 \mathrm{ft}^{2}$

Caloria HT-43

Inlet $392^{\circ} \mathrm{F}$, outlet $550^{\circ} \mathrm{F}$

$\mathrm{q}_{\mathrm{i}}=190 \mathrm{Btu} / \mathrm{ft}^{2} \cdot \mathrm{h}$

$\dot{\mathrm{m}}=15,8001 \mathrm{~b} / \mathrm{h}$

Subsystem efficiency at summer solstice $=38.68$

\section{Thermal storage}

Type :

Tank size:

Storage temperature:

Storage medium:

Insulation:
Stratified liquid (thermocline)

50,000 gal - $13.67-\mathrm{ft}$ diameter by 49-ft length $(30,000$ gal usable storage)

$392^{\circ} \mathrm{F}$ to $550^{\circ} \mathrm{F}$

Caloria HT-43

12-in.-thick fiberglass

\section{Cooling system}

Type :

Water (makeup):

Condensing temperature:

\section{Power Generation}

Type: :

Working fluid:

Gross efficiency:
Vapor condenser

$10 \mathrm{gal} / \mathrm{min}$

$105^{\circ} \mathrm{F}$

Organic Rankine Cycle

Toluene

208 
Available from

National Technical Information Service

U.S. Department of Commerce

5285 Port Royal Road

Springfield, VA 22161

Price: printed copy $\$ 4.50 ;$ microfiche $\$ 3.00$ 
OVERALL SUMMARY

\section{System Performance}

The final product of the facility is the electrical power it feeds into the grid network of the local utility. The monthly electrical energy generation for January through August 1980 is shown in Figure 1 .

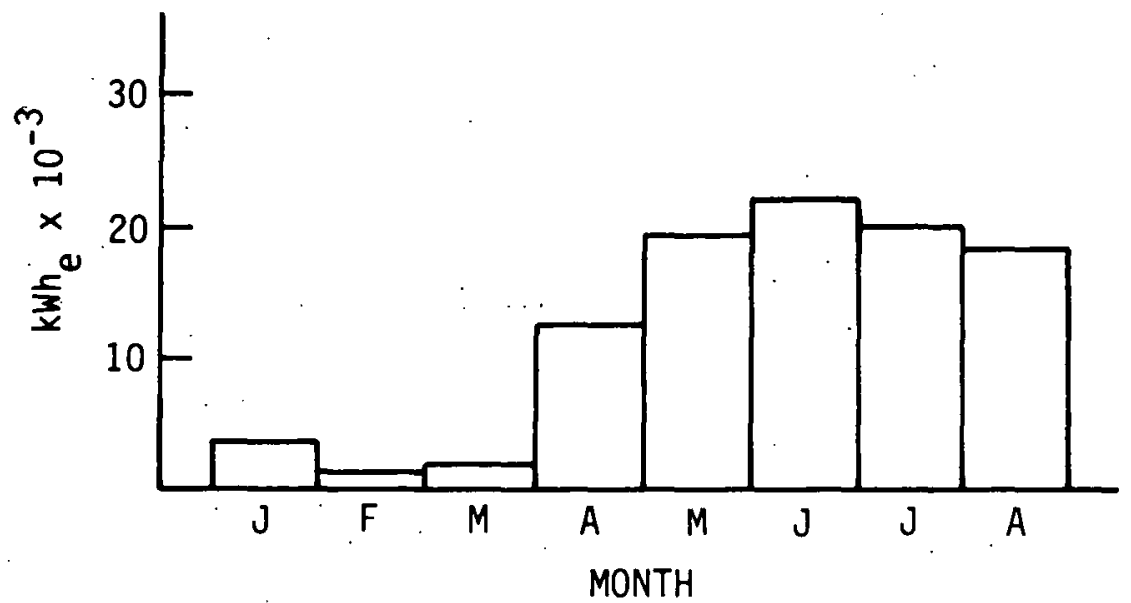

Figure 1. Electrical Energy Generated by the Plant from January through August 1980

The line-focus solar collectors were oriented in the north-south direction to maximize the amount of energy collected in the summer when irrigation dcmands are the highest. This orientation results in reduced energy collection in the winter. The present use of coilzak as the reflector material for the solar collectors contributes to the poor winter performance due to the material's low reflectivity. Yearround system performance can be improved by the use of better reflector materials. Most of the electrical energy generated in Tanuary and 
February resulted from operation of the gas-fired heater. Energy production in February and March was abnormally low due to problems with the pump seals in the collector field pump and turbine pump.

\section{Operation and Recurring Maintenance Costs}

The operation and recurring maintenance costs have been broken down into weekly costs for summer and winter. The costs assume the facility is operated a full 7 days a week.

The operational costs include the man-hours required to initiate. facility operation daily and to monitor the operation in order to assure all is normal. The power conversion system has required operator attention during startup and some manual control. Direct operational costs have included cooling water for the turbine's condenser, nitrogen for the expansion volume on top of the thermal storage tank, $\mathrm{CO}_{2}$ for cooling the pump seals, and electriclty for air conditioning the control room.

The labor for operation amounted to 30 hours per week. The direst rosts are itemized below.

\section{Weekly Costs of Plant Operation}

\begin{tabular}{lrr}
\multicolumn{1}{c}{ Operation Components } & $\frac{\text { Sumer }}{320}$ & Wintar \\
Water (municipal) & 35 & 5 \\
Water treatment & 5 & 10 \\
$\mathrm{~N}_{2}$ & 8 & 5 \\
$\mathrm{CO}_{2}$ & $\frac{10}{578}$ & -- \\
Electricity (air conditioning) & $\$ 26$. \\
Total cost per week
\end{tabular}

The recurring maintenance costs include labor and the cost of supplies and replacement materials for those efforts. Below is a summary of the average weekly costs over the year broken down for the various subsystems. 
Weekly Costs of Recurring Maintenance

\begin{tabular}{lcc}
\multicolumn{1}{c}{ Subsystem } & $\frac{\text { Man-Hours }}{8.0}$ & $\frac{\text { Materials }}{\$ 15.20}$ \\
Solar collector & 4.8 & 1.60 \\
Fluid loops & $\frac{4.8}{17.6}$ & $\frac{15.90}{\$ 32.70}$ \\
Power conversion & Total cost per week &
\end{tabular}

\section{Experiences and Insights}

Summarized below are the lessons learned from the construction and operation of the facility.

Construction:

- Piping joints will tend to leak, with threaded joints being the worst, followed by flanges, then swagelock fittings, then welded joints.

- Conventional arc welding of plumbing joints is satisfactory in most cases. Tungsten inert gas (TIG) welding is necessary for stainless-steel attachments and swagelock thermocouple fittings.

- Thermocouples with swagelock fittings are best for measuring. fluid temperatures.

- Ail valve bodies should be welded: into their pipelines.

- Insulation should be installed in multilayers with lapped joints.

- Valve stems should point downward to prevent leakage from getting into insulation.

- Manholes on the side of a thermal storage tank are undesirable since they will leak fluid and are a source of heat loss.

- Leak tests should be performed on the pipelines with the lines filled with fluid and at temperature prior to insulating them.

- Operating personnel should be onsite during final construction and checkout. 


\section{Operation:}

- Decomposition of the Caloria HT-43 has been very slight.

- The automatic fill system for the storage tank has not been needed since fluid decomposition was slight.

- Eighty percent of nonrecurring maintenance work has been on the power generation subsystem.

- A rain switch has been installed to allow the operators to point the collectors upward during a rainstorm.

- To prevent thermosiphoning, plumb downward away from heat sources.

- Provide an automatic closure valve in the pipeline to the base of the thermal storage tank to prevent a large oil spill.

- Provide easy, year-round access to all subsystems.

- Provide an evacuation route from potential oil spill areas.

- Construct an earth berm around the thermal storage tank.

- Provide a well-marked, accessible "kill button" to deactivate valves, collectors, flow, etc., in event of an emergency.

- Use water--not $\mathrm{CO}_{2}$ or chemicals--on oil fires.

- Repair oil leaks on a priority basis.

- Avoid overheating oil seals on pumps, etc.

- Label all fluid containers carefully and maintain tight control.

- Provide a backup electrical power source to allow the collectors to be defocused in the event of a commercial power outage.

- Forbid the bypassing of safety devices.

- Set up extensive, periodic, preventive inspection and maintenance.

- Maintain a good spare-parts inventory.

- Periodically tighten flanges. 
- Clean receiver tubes weekly.

- A collector field temperature-control system which senses collector outlet oil temperature at only one point works well.

- Collector field startup in cold weather using warm weather techniques has proven to be no problem.

\section{Future Plans}

During the coming year, system performance will continue to be monitored. In addition, the remaining specific tests in the test plan will be conducted. Some changes will be made to the facility to upgrade its performance and eliminate some of its problems. These changes will include the installation of FEK-244 reflective surfaces on the collectors and modifications to the plumbing system. The replacement of the present Coilzak reflector material with FEK-244 will improve the reflectivity of the collector surfaces from $60 \%$ for the Coilzak to 858 for the FEK-244 when the surfaces are clean. A significant improvement in system performance is anticipated with the FEK-244.

The plumbing modifications will include ( 1 ) the elimination of the buffer tank at the inlet to the collector field pump, (2) the replacement of the present three-way butterfly-type diverter valve with a conventional spool-type three-way diverting valve, and (3) the plumbing of the gas-fired heater in series with the collector field. The elimination of the $1.89-\mathrm{m}^{3}$ (500-gallon) buffer tank will el iminate a source of heat loss and should shorten the warmup time of the collector field. The new three-way valve will eliminate the leakage problems experienced with the old valve. The old valve was allowing about $0.6 \mathrm{l} / \mathrm{s}$. ( $10 \mathrm{gal} / \mathrm{min})$ of oil to be diverted to the top of the sturage tank during warmup, when the oil is being circulated through the collector field. With the gas-fired heater operating in series with the collector field, it will be possible to generate electrical power throughout the winter season, and plans are being made to do so. In this mode, the collector field will be used to preheat the Caloria. 
Operation and recurring maintenance costs will be watched during the coming year, with an eye toward minimizing them. With cost minimization in mind, further steps will be taken toward complete automation of the facility.

Grain alcohol production processes and hardware are being studied to determine the feasibility of installing a facility onsite for producing alcohol to fuel farm machinery; the alcohol would be produced utilizing a portion of the energy collected by the the solar collectors. 
ENERGY COLLECTION AND PRODUCTION OF THE COOLIDGE, ARIZONA, SOLAR IRRIGATION FACILITY FOR THE PERIOD 1 JANUARY 1980 THROUGH 31 AUGUST 1980

The amounts of available solar energy, collected thermal energy, and generated electrical energy have been compiled for January through August 1980. Solar energy data for intensities greater than $300 \mathrm{~W} / \mathrm{m}^{2}$ (946 Btu/ft.h) integrated over the whole day have been compiled. That portion of the total direct radiation received during collector system operation is listed as solar energy available during operation. The collected solar energy is the daily thermal energy output of the solar collector subsystem. Electrical energy and natural gas usage for. plant operation and tests have also been recorded. For comparison with plant production, the quantity of electricity used by three irrigation pumps on the Dalton Cole farm has been obtained. The three pumps require approximately $150 \mathrm{~kW}(200 \mathrm{hp})$.

Energy data for the 8 months are attached. When unavailable due to data gathering problems, the information has been estimated and is noted. Footnotes explain the estimation methods: Monthly totals for available solar energy and collected thermal energy are presented graphically in Figure 1. Total electrical energy generated monthly is shown in Figure 2 .

A relatively small amount of thermal energy was collected in January, February, and March. The plant was not operated on 1 January, and 15 other days received little direct solar radiation. Much of the energy collected between the first of January and the middle of February was too low in temperature for power production. The reason for this was the lower solar collector efficiency at this time of the year, which was caused by the low sun angle. This seasonal low efficiency is a characteristic common to all collector arrays oriented in 
the north-south direction. Collector field pump repair caused the February energy collection to be very low and greatly reduced the March operation. The long downtime was caused by heavy rains which resulted in deep mud around the pump. Because of the mud, a boom truck could not be driven in to remove the heavy pump for repair. To avoid long downtimes due to pump failures in the future, a portable hoist is kept onsite along with a spare pump impeller, shaft, bearing, and seal assembly for quick repair. After March, thermal energy collection gradually increased, peaking in May and June. May generally has less cloudy weather than June. Energy collection decreased in July and August; the decline was accentuated by the number of days receiving reduced amounts of direct solar radiation during those months.

The amount of collected thermal energy as a percentage of available direct radiation received during collector system operation increased from 7.58 in January to 20.68 in March to 32.58 in June.

Percent of Available Solar Radiation During Operation Collected as Thermal Energy

$\begin{array}{lrlr}\text { January } & 7.5 & \text { May } & 30.6 \\ \text { February } & 9.7 & \text { June } & 32.5 \\ \text { March } & 20.6 & \text { July } & 32.3 \\ \text { April } & 28.2 & \text { August } & 29.6\end{array}$

Electrical energy production averaged about $815 \mathrm{kWh}\left(2.78^{\circ} \mathrm{x}\right.$ $10^{6} \mathrm{Btu}$ ) per operating day in June. Power plant electrical energy use averaged about $260 \mathrm{kWh}\left(8.87 \times 10^{5} \mathrm{Btu}\right)$ on those same days. The ratio of net to gross energy production increases with increased operating hours. Natural gas was used for winter operation and spec1flc power plant tests. Spring and summer usage occurred during periodic boiler tests.

Irrigation pumps were operated periodically during the early part of the year. However, almost continuous pump operation was recorded during some periods of the spring and summer seasons. 
Plant performance and data gathering efforts improved during the year. Eńergy budgets will be compiled during 1980-81 to provide better data for use in analysis of performance.

\section{Conclusions}

This section has presented the energy production records for the facility to date. These records illustrate how energy production varies from season to season with a parabolic trough collector system displaying the collectors in the north-south orientation. Had the collectors been oriented in the east-west direction, electrical power production would have been possible year-round; however, production in the summer would have been significantly less. The north-south orientation was chosen to maximize electrical power production in the summer when power consumption by the irrigation pumps is at its peak. 
-50 kWe SOlar Project

MONTILY ENERGY BALANCE

\begin{tabular}{|c|c|c|c|c|c|c|c|c|c|c|c|}
\hline \multirow{3}{*}{ Day } & \multicolumn{3}{|c|}{ OPERAT1NG TIME, hr. } & \multirow{2}{*}{\multicolumn{5}{|c|}{$\begin{array}{l}\text { TIIERMAL ENERGY, kw/ } \\
\text { SOLAR ENERGY }\end{array}$}} & & & \\
\hline & \multirow{2}{*}{$\begin{array}{l}\text { Solar } \\
\text { Energy } \\
\text { Avallable } \\
\end{array}$} & \multirow[b]{2}{*}{$\begin{array}{l}\text { C-llector } \\
\text { System }\end{array}$} & \multirow[b]{2}{*}{$\begin{array}{l}\text { Generator } \\
\text { System }\end{array}$} & \multicolumn{2}{|c|}{ SOLAR ENERGY } & & & & \multicolumn{2}{|c|}{ ELECTRICAL ENERGY, KWWI } & \multirow{2}{*}{$\begin{array}{l}\text { Irrtgat lon } \\
\text { Pumip } \\
\text { Energy } \\
\text { Usnge. } \\
\text { kwh } \\
\end{array}$} \\
\hline & & & & $\begin{array}{l}\text { Total } \\
\text { D1 rect }\end{array}$ & $\begin{array}{l}\text { Avallable } \\
\text { Durtng } \\
\text { Operattan b }\end{array}$ & Collected & $\begin{array}{l}\text { Natural } \\
\text { Gaq } \\
\text { Use }\end{array}$ & $\begin{array}{l}\text { Total } \\
\text { Input }\end{array}$ & $\begin{array}{l}\text { Generatur } \\
\text { Output }\end{array}$ & $\begin{array}{l}\text { Plant } \\
\text { Usane }\end{array}$ & \\
\hline 1 & 7.0 & -- & $-\cdots$ & $6500^{c}$ & $6240^{\circ}$ & --- & --- & ---- & -- & 60 & --- \\
\hline 2 & 7.3 & 0.8 & --- & $6600^{c}$ & ---- & ---- & 7028 & 7028 & -- & 90 & ---- \\
\hline 3 & 7.5 & 7.5 & --- & 6675 & 6511 & $456^{e}$ & $--\cdots$ & $\dot{456}$ & -- & 100 & $\cdots$ \\
\hline 4 & 7.5 & 7.5 & --- & 7322 & 6956 & $487^{e}$ & 666 & 1153 & --- & 120 & --- \\
\hline 5 & 7.5 & 5.5 & --- & 5185 & 4906 & $319^{e}$ & --- & 319 & -- & 90 &.-- \\
\hline 6 & 0 & -- & --- & 2697 & ---- & $\cdots$ & 6664 & 6664 & --- & 80 & --- \\
\hline 7 & o. & --- & --- & 1942 & ---- & --- & 10117 & 10117 & -- & 200 & ---- \\
\hline 8 & 0 & --- & --- & 3216 & ----- & --- & 1149 & 7149 & --- & 90 & $-\cdots$ \\
\hline 9 & 0 & -- & -- & 68 & ----- & $\because--$ & 5695 . & 5695 & --- & $90^{\circ}$ & ---- \\
\hline 10 & 0 & --- & --- & 33 & $\cdots$ & --- & -- & ----- & -- & 70. & --- \\
\hline 11 & 0 & -- & 2.3 & 226 & --- & ---- & ---- & ---- & 360 & 1.40 & .--- \\
\hline 12 & 3.0 & 3.0 & --- & 6453 & 5994 & --- & $-\cdots$ & ----- & -- & 80 & --- \\
\hline 13 & 7.0 & 7.0 & $-n$ & $1312 E$ & 12669 & 511 & ----- & 511 & --- & 90 & ---- \\
\hline 14 & 0.8 & 0.8 & 2.2 & $379]$ & 1807 & --- & 4483 & 4483 & 256 & $160^{\circ}$ & --- \\
\hline 15 & 7.0 & 7.0 & -- & $1182:$ & 11110 & 644 & ---- & 644 & -- & 90 & --- \\
\hline 16 & 7.5 & 7.5 & -- & 15279 & 14758 & 1353 & ---- & 1353 & -- & 100. & --- \\
\hline 17 & 3.5 & 3.5 & --- & 8617 & 8157 & 641 & ----- & 641 & -- & 80 & --- \\
\hline 18 & 0 & --- & 1.5 & 6752 & $-\cdots$ & --- & 7149 & 7149 & 224 & 140 & --- \\
\hline 19 & 3.2 & 3.2 & --- & 4063 & 3075 & 128 & $\cdots$ & 128 & -- & 90 & --- \\
\hline 20 & 7.5 & 7.5 & --- & 14374 & 13620 & 1587 & ----- & 1587 & --- & 90 & --- \\
\hline 21 & 0 & --- & 3.2 & 1252 & $\cdots$ & ---- & 5271 & 5271 & 576 & 180 & --- \\
\hline 22 & 7.5 & 7.5 & -- & 8691 & 5824 & 338 & ---- & 338 & --- & 100 & $-\cdots$ \\
\hline 23 & 7.5 & 7.5 & --- & $8700^{c}$ & $8352^{d}$ & $668^{e}$ & --- & 668 & --- & 90 & -- \\
\hline 24 & 7.7 & 7.7 & -- & $12500^{c}$ & $12000^{d}$ & $1080^{e}$ & $--\cdots$ & 1080 & --- & 100 & -- \\
\hline 25. & 8.1 & 8.1 & 0.7 & $15000^{c}$ & $14400^{d}$ & $1440^{e}$ & ----- & 1440 & 96 & 120 & $-\cdots$ \\
\hline 26 & 8.0 & 8.0 & -- & $14000^{c}$ & $13440^{d}$ & $1344^{e}$ & ----- & 1344 & -- & 90 & --- \\
\hline 27 & 7.8 & 7.8 & --- & $13500^{c}$ & $12960^{d}$ & $1231^{e}$ & ----- & 1231 & $\therefore-$ & 90 & $\because-$ \\
\hline 28 & 0 & --- & --- & $2000^{c}$ & ----- & ---- & 8210 & 8210 & $-\cdots$ & 90 & 1140 \\
\hline 29 & 0 & -- & 3.8 & $3000^{C}$ & ----- & --- & 8906 & 8906 & 736 & 210 & 11.40 \\
\hline 30 & 2.5 & 2.5 & 4.4 & $5000^{\circ}$ & $4800^{d}$ & $360^{e}$ & 9754 & 10114 & 640 & 230 & 1140 \\
\hline 31 & 6.0 & 3.5 & 2.3 & $10000^{c}$ & $9600^{d}$ & $768^{e}$ & 2484 & 3252 & 384 & 160 & 1140 \\
\hline TOTAL & 124.4 & 113.4 & 20.4 & 217989 & 177179 & 13355 & 83576 & 96931 & 3272 & $3410^{1}$ & $4560 \mathrm{~B}$ \\
\hline
\end{tabular}

Note: See page 32 for fjotnotes.

. 
150 k the SOLAR PROJECT

MONTHLY ENERGY BALANCE

\begin{tabular}{|c|c|c|c|c|c|c|c|c|c|c|c|}
\hline \multirow{3}{*}{ Day } & \multicolumn{3}{|c|}{ OPERATING THE, hr. } & \multirow{2}{*}{\multicolumn{5}{|c|}{$\begin{array}{l}\text { THERMAL ENERGY, KWh } \\
\text { SOLAR EHERGY }\end{array}$}} & & & \\
\hline & \multirow{2}{*}{ 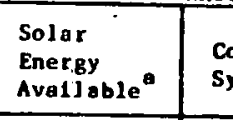 } & \multirow[b]{2}{*}{$\begin{array}{l}\text { Collector } \\
\text { Systen }\end{array}$} & \multirow[b]{2}{*}{$\begin{array}{l}\text { Generat or } \\
\text { System }\end{array}$} & & & & & & \multicolumn{2}{|c|}{ ELECTRICAL ENERGY, KWh- } & \multirow{2}{*}{$\begin{array}{l}\text { Jrrigat Ion } \\
\text { Pump } \\
\text { Energy } \\
\text { Usnge. } \\
\text { KWWl }\end{array}$} \\
\hline & & & & $\begin{array}{l}\text { Total } \\
\text { D1 rect }\end{array}$ & $\begin{array}{l}\text { Avallable } \\
\text { During } \\
\text { Operaklan }\end{array}$ & Collected & $\begin{array}{l}\text { Natural } \\
\text { Gas } \\
\text { Use }\end{array}$ & $\begin{array}{l}\text { Total } \\
\text { Input } 1\end{array}$ & $\begin{array}{l}\text { Generator } \\
\text { Output }\end{array}$ & $\begin{array}{l}\text { Plent } \\
\text { Usege: }\end{array}$ & \\
\hline 1 & 7.8 & & -- & $14000^{c}$ & $13440^{d}$ & $1344^{e}$ & $\cdots$ & 1344 & -- & 80 & $114 \theta:$ \\
\hline 2 & 3.6 & 7.6 & -- & $13500^{c}$ & $12960^{d}$ & $1296^{e}$ & $-\cdots$ & .1296 & $=-$ & 80 & 1160 \\
\hline 3 & 6.8 & 6.8 & $\cdots$ & $12500^{c}$ & $12000^{d}$ & $1200^{e}$ & $-\cdots$ & 1200 & -.- & $80^{\circ}$ & 1140 \\
\hline 4 & 7.8 & 7.8 & - & $7500^{\circ}$ & $7200^{d}$ & $684^{e}$ & $\cdots$ & 684 & -- & 80 & 1140 \\
\hline 5 & 8.2 & 8.2 & $\because$ & $9000^{c}$ & $8640^{d}$ & $820^{e}$ & ---- & 820 & --- & 80 & --- \\
\hline 6 & 7.2 & 7.2 & $\cdots$ & $6500^{c}$ & $6240^{d}$ & $562^{e}$ & $-\cdots$ & 562 & --- & 80 & $-\cdots$ \\
\hline 7 & 8.2 & 8.2 & -- & $13500^{c}$. & $12960^{d}$ & $1296^{\mathrm{e}}$ & --- & 1296 & -- & 90. & $-\cdots$ \\
\hline 8 & 0. & $\rightarrow-$ & -- & $2500^{c}$ & $\therefore-$ & --- & - - & --.-- & -- & 60 & $\therefore$ \\
\hline 9 & 8.3 & 8.3 & - & $14000^{c}$ & $13440^{d}$ & $1344^{\mathrm{e}}$ & $-\cdots$ & 1344 & -- & 90 & $-\cdots$ \\
\hline 10 & 8.5 & 8.5 & - & $12500^{c}$ & $12000^{d}$ & $1200^{\mathrm{e}}$ & $-\cdots$ & 1200 & --- & 90 & --- \\
\hline 11 & 8.1 & 8.1 & 4.9 & $10000^{c}$ & $.9600^{d}$ & $768^{e}$ & - & 768 & 530 & 240 & --- \\
\hline 12 & 8.1 & $\cdots$ & -- & 8419 & $\cdots$ & -- & 11753 & 11753 & --- & 100 & $-\cdots$ \\
\hline 13 & 0 & -- & 2.4 & 33 & ---- & --- & $-\cdots$ & --- & 220 & 130 & $\therefore-$ \\
\hline 14 & 0 & $\mapsto-$ & 2.3 & 1437 & $-\cdots$ & $\cdots$ & $\cdots$ & $\cdots$ & 270 & 130 & --- \\
\hline 15 & 0 & $\cdots$ & $\therefore$ & 799 & $\cdots$ & --- & $--\div-\therefore$ & $-\cdots$ & -- & 60. & ---- \\
\hline 16 & 0 & -- & -- & 1919 & $\cdots$ & --- & ---- & -...- & $-\cdots$ & 60 & ---- \\
\hline 17 & 0 & $-\cdots$ & --- & 1085 & $\cdots$ & $\cdots$ & $\cdots-$ & :---- & -- & 60 & $\cdots$ \\
\hline 18 & 0 & -- & --- & 40 & ---- & --- & $-\cdots$ & $\cdots$ & --- & 50 & --- \\
\hline 19 & 0 & -- & -- & SO7 & $-\cdots$ & $-\cdots-$ & $\cdots$ & --- & $--\because$ & 60 & --- \\
\hline 20 & 2.5 & -- & -- & 9831 & $\cdots$ & ---- & ---- & $=-\infty$ & --- & 60 & $\cdots$ \\
\hline 21 & 0 & -- & $\cdots$ & 561 & $-\infty$ & $\cdots$ & ---- & $\cdots$ & --- & 60 & --- \\
\hline 22 & 8.6 & - & -- & 146595 & $\cdots$ & -- & $\cdots$ & $\cdots$ & -- & 60 & $\cdots$ \\
\hline 2.3 & 8.8 & -- & -- & 18394 & $\cdots$ & -- & -.-- & $-\cdots$ & $\cdots$ & 50 & --- \\
\hline 24 & 8.8 & $\cdots$ & $\cdots$ & 12519 & - & --- & $-\cdots$ & --- & --- & 70 & --- \\
\hline 25 & 9.0 & -- & -- & 1.6454 & $\cdots$ & -- & ---- & $-\cdots$ & --- & 60 & $-\cdots$ \\
\hline 26 & 9.0 & $-\therefore$ & --- & 21609 & $\cdots$ & $\cdots$ & $\because--$ & -.-- & -- & 60 & 2182 \\
\hline 27 & 9.0 & $\cdots$ & -- & 18925 & $-\cdots$ & --- & --- & $\cdots$ & $\cdots$ & 60 & 2182 \\
\hline 28 & 9.0 & -- & $\because-$ & 13301 & $\cdots$ & -- & --- & $\cdots$ & --- & 60 & 2182 \\
\hline 29 & 9.0 & -- & --- & 9832 & --.- & $\because$ & $\cdots$ & ---- & -- & 60 & 2182 \\
\hline $\begin{array}{l}30 \\
31\end{array}$ & . & & & & & & & & . & & \\
\hline TOTAL & 160.2 & 78.5 & 9.6 & 319826 & 108480 & 10514 & 11753 & 22267 & & & \\
\hline & & & & & & & & 22267 & 1020 & $2300^{f}$ & $13288^{8}$ \\
\hline
\end{tabular}


15C. Wha SOIJR PROJECT

MONTHLY ENERCY BALANCE

\begin{tabular}{|c|c|c|c|c|c|c|c|c|c|c|c|}
\hline \multirow{3}{*}{ Day } & \multicolumn{3}{|c|}{ OPERATIME TIME, hr. } & \multirow{2}{*}{\multicolumn{5}{|c|}{ THERMAL ENERGY, KWW }} & \multirow{2}{*}{\multicolumn{2}{|c|}{ ELECTRICAI. ENERIYY, KWhI }} & \\
\hline & \multirow{2}{*}{$\begin{array}{l}\text { Solar } \\
\text { Energy } \\
\text { Avallahle }\end{array}$} & \multirow[b]{2}{*}{$\begin{array}{l}\text { Co:lector } \\
\text { System }\end{array}$} & \multirow[b]{2}{*}{$\begin{array}{l}\text { Generator: } \\
\text { System: }\end{array}$} & \multicolumn{2}{|c|}{$\begin{array}{l}\text { SOLAR ENERGY } \\
\text { Avallable }\end{array}$} & & & & & & \multirow{2}{*}{$\begin{array}{l}\text { Irrigeclan } \\
\text { Pump } \\
\text { Energy } \\
\text { Usoge. } \\
\text { LWh }\end{array}$} \\
\hline & & & & $\begin{array}{l}\text { Total } \\
\text { Di rect }\end{array}$ & $\begin{array}{l}\text { Avallable } \\
\text { During } \\
\text { Operation }\end{array}$ & Collected & $\begin{array}{l}\text { Nat ural. } \\
\text { Gas } \\
\text { Use }\end{array}$ & $\begin{array}{l}\text { To: al } \\
\text { Insut } 1\end{array}$ & $\begin{array}{l}\text { Generator } \\
\text { Output }\end{array}$ & $\begin{array}{l}\text { Plant } \\
\text { Uas }\end{array}$ & \\
\hline 1 & $\mathbf{0}$ & -+ & -- & $51 ; 3$ & --- & --- & ---- & ----- & --- & 70 & 2182 \\
\hline 2 & $\mathbf{0}$ & -- & $\cdots$ & $60: 7$ & $--\infty$ & --- & ---- & $-\cdots$ & -- & 70 & 2182 \\
\hline 3 & 0 & -- & --- & 2439 & ---- & --- & ---- & $\ldots-$ & -- & 70 & $2182^{\circ}$ \\
\hline 4 & 4.5 & -- & $\because-$ & 14303 & $-\cdots$ & $-\cdots$ & ----- & -..... & --- & 70 & \\
\hline 5 & 8.3 & -- & -- & $19 \geq 24$ & ---- & -- & $-\cdots$ & ---- & -- & 70 & 480 \\
\hline 6 & 7.0 & $-\cdots$ & $\cdots$ & 14930 & -..-- & --- & ----- & $-\cdots$ & --- & 10 & 480 \\
\hline 1 & 9.0 & 3.2 & -- & $14 \geq 20$ & $-\cdots$ & ---- & ----- & $\ldots$ & --. & 70 & .480 \\
\hline 8 & 9.3 & 8.8 & -- & 16205 & --.-- & --- & - & ----- & -- & 80 & 480 \\
\hline 9 & 8.0 & 8.0 & -- & $11: 16$ & $\cdots$ & ---- & $-\ldots$ & - & $\because$ & 100 & 480 \\
\hline 10 & o & --- & -- & 60 & -..- & -- & --_-- & - & $\cdots$ & 90 & 480 \\
\hline 11 & 8.0 & 2.5 & - & 10341 & 9323 & 611 & $\ldots$ & 611 & -- & 70 & 2419 \\
\hline 12. & 9.6 & 9.6 & -- & $2 \pm=84$ & 15398 & 3724 & - & & $\therefore$ & 80 & 2419 \\
\hline 13 & 9.7 & 2.1 & - & $19: 016$ & 6527 & 1217 & -- & $\begin{array}{l}3: 24 \\
1247\end{array}$ & --- & 100 & 2419 \\
\hline 14 & 9.7 & --- & - & $18: 50$ & --- & ---- & ----- & $\begin{array}{l}1247 \\
-\ldots---\end{array}$ & -- & 80 & 2419 \\
\hline 13 & 0 & -- & -- & 7450 & $-\cdots$ & -..- & -- & $-\cdots$ & $\cdots$ & 70. & 2411 \\
\hline 16 & 9.8 & 9.8 & $\because-$ & 19577 & 12536 & 2152 & ---- & --- & $\cdots$ & 70 & 2411 \\
\hline 17 & 9.7 & 0.7 & -- & 2:D26 & 17929 & 167 & 8603 & 2152 & --- & 100 & .2411 \\
\hline 18 & 9.1 & 7.8 & -- & 15944 & 14858 & 805 & -0003 & 8770 & $\therefore-$ & 100 & 2411 \\
\hline 19 & 2.3 & 2.3 & --- & $1474 \varepsilon$ & 10340 & 2378 & --.- & 805 & $\cdots$ & 100 & 2411 \\
\hline 20 & 9.8 & 9.3 & -- & 2043 & 19645 & 5246 & ----- & 2078 & --- & 80 & 2411 \\
\hline 21 & 9.7 & 3.5 & -- & $1: 69 ?$ & 8470 & 84 & ---- & 5246 & --- & 100 & 2411 \\
\hline 22 & $\mathbf{0}$ & -- & --- & 15618 & -.... & $-\cdots$ & $\cdots$ & 84 & - & 80 & 2411 \\
\hline 23 & 10.1 & 10.1 & -- & 10947 & 17715 & 5525 & ---- & $\rightarrow$ & -- & 70 & 2411 \\
\hline 24 & 5.8 & 5.8 & 3.1 & 10041 & 5195 & 1231 & $\cdots$ & $\leq 925$ & -- & 100 & 2411 \\
\hline 25 & 7.2. & 1.2 & 1.5 & 15530 & 12265 & 2575 & $\cdots$ & 1231 & 220 & 180 & 2411 \\
\hline 26 & 1.2 & 1.2 & 2.4 & $\Xi \pm 57$ & --.-- & $\ldots$ & 5574 & 2149 & 240 & 160 & 5896 \\
\hline 27 & 8.8 & 8.2 & 2.3 & $10503^{e}$ & $15510^{d}$ & $4188^{e}$ & 9148 & $\$ 148$ & 180 & 180 & 5896 \\
\hline 28 & 10.0 & 10.0 & 1.3 & $=1500^{C}$ & $20855^{d .}$ & $6674^{e}$ & ----- & -188 & 290 & 160 & $5896^{\circ}$ \\
\hline 29 & 9.8 & 1.6 & --- & $20500^{c}$. & $2000^{d}$ & $480^{\circ}$ & ---- & 6674 & 180 & 140 & 5892 \\
\hline 30. & 9.9 & 9.9 & $\cdots$ & $7000^{c}$ & $20370^{d}$ & $6111^{e}$ & $\cdots$ & i 80 & -- & 60 & 5892 \\
\hline $3 !$ & 9.7 & 0.5 & 2.7 & 22749 & 480 & 66 & $-\cdots$ & 3111 & -- & 100 & 2591 \\
\hline TOTAI. & 206.0 & 122.7 & 13.3 & \#SE6I & 209416 & 67234 & & 36 & & .150 & .2591 \\
\hline & & & & & & 43234 & 23325 & 66559 & 1440 & $3040^{k}$ & $80373^{6}$ \\
\hline
\end{tabular}


150 KHE SOLAR PROJECT

HOMTHLY ENERGY BALANCE

\begin{tabular}{|c|c|c|c|c|c|c|c|c|c|c|c|}
\hline \multirow{3}{*}{ Day } & \multicolumn{3}{|c|}{ OYERATIRE TUE, hr. } & \multirow{2}{*}{\multicolumn{5}{|c|}{$\begin{array}{l}\text { TUERMAL ENERCY, K/m } \\
\text { SOLAR ENERCY }\end{array}$}} & \multirow{2}{*}{\multicolumn{2}{|c|}{ ELECTRICAL ENERGYY, $\mathrm{kHH}$}} & \multirow{3}{*}{$\begin{array}{l}\text { Irriget Ion } \\
\text { Punp. } \\
\text { Enerigy } \\
\text { Ugege. } \\
\end{array}$} \\
\hline & \multirow{2}{*}{$\begin{array}{l}\text { Solar } \\
\text { Energy } \\
\text { Avolloble }\end{array}$} & \multirow[b]{2}{*}{$\begin{array}{l}\text { Collector } \\
\text { syoten }\end{array}$} & \multirow[b]{2}{*}{$\begin{array}{l}\text { Cenerator } \\
\text { Sybten }\end{array}$} & & & & & \multirow[b]{2}{*}{$\begin{array}{l}\text { Total } \\
\text { Input } 1\end{array}$} & & & \\
\hline & & & & $\begin{array}{l}\text { Total } \\
\text { D1 rect }\end{array}$ & $\begin{array}{l}\text { Avallable } \\
\text { During } \\
\text { Operntion }\end{array}$ & Collected. & $\begin{array}{l}\text { Nat ural } \\
\text { Gas i } \\
\text { Use h } \\
\end{array}$ & & $\begin{array}{l}\text { Generator } \\
\text { Output }\end{array}$ & $\begin{array}{l}\text { Plant } \\
\text { Uange }\end{array}$ & \\
\hline 1 & 7.1 & 7.1 & $\because 1.6$ & 14421 & 11969 & 3380 & $-\infty$ & 3388 & 288 & 140 & 2591 \\
\hline 2 & 4.7 & 4.7 & 1.3 & 12765 & 8201 & 1882 & $\cdots$ & 1882 & 160 & 120 & 2591 \\
\hline $\mathbf{3}$ & 10.4 & 10.4 & 4.0 & 21417 & 18463 & 6324 & $-\infty$ & 6324 & 800 & 100 & 2591 \\
\hline 4 & 7.3 & 7.3 & .-- & 14955 & 12031 & 3294 & $-\cdots$ & 3294 & -- & 110 & 2591 \\
\hline 3 & 10.4 & 10.4 & 3.6 & 19478 & 17891 & 4817 & $\ldots$ & 4817 & 736 & 300 & 3672 \\
\hline 6 & 10.6 & 10.6 & - & 15735 & $14688^{\circ}$ & 3308 & $-\infty$ & 3308 & --- & 90 & 3672 \\
\hline 1 & $\therefore 10.7$ & 10.2 & 5.6 & 17877 & 16895 & 5273 & $\cdots$ & 5273 & 960 & 200 & 3672 \\
\hline 8 & $\ldots 10.6$ & 10.6 & -- & 20273 & 18864 & 6429 & --- & 6429 & $-\div-$ & 330 & 3672 \\
\hline 9 & 10.7 & 10.7 . & 5.6 & 20569 & 19113 & 4048 & $-\cdots$ & 4048 & 1184 & 270 & 3672 \\
\hline 10 & 10.7 & 10.7 & $\because$ & $20500^{\mathrm{C}}$ & $19885^{d}$ & $6761^{e}$ & $\cdots$ & 6761 & -- & 20 & 3672 \\
\hline 11 & 10.8 & 10.8 & 6.3 & $17500^{c}$ & $13125^{d}$ & $4069^{e}$ & $\cdots$ & 4069 & 1216 & 210 & 3672 \\
\hline 12 & 11.0 & 8.5 & 5.7. & $16000^{c}$ & $12000^{d}$ & $3360^{\mathrm{e}}$ & 3817 & 7177 & 1024 & 190 & 3672 \\
\hline 13 & 10.8 & 9.8 & -- & $16500^{c}$ & $15675^{d}$ & $4546^{\mathrm{e}}$ & --- & 4546 & -- & $180^{\circ}$ & 3846 \\
\hline 16 & 10.8 & 10.8 & 5.3 & $19000^{c}$ & $18240^{d}$ & $5837^{e}$ & ---- & 5837 & 1280 & 320 & 3846 \\
\hline 15 & 10.8 & 1.5 & -- & $4000^{c}$ & $3960^{d}$ & $870^{\mathrm{e}}$ & 5937 & 6807 & -- & $180^{\circ}$ & 3846 \\
\hline 16 & 11.0 & 10.3 & 5.0 & 16395 & 16204 & 4481 & 666 & 5147 & 704 & 230 & 3846 \\
\hline 17 & 10.8 & 1.3 & 2.5 & 11748 & 11730 & 3450 & $\therefore--$ & 3450 & 320 & 150 & 3846 \\
\hline 18 & 10.9 & 10.9 & 2.7 & 17018 & 16273 & .5320 & ---- & 5320 & 416 & 160 & 3846 \\
\hline 19 & 10.9 & 10.9 & 2.9 & 16754 & 15814 & 5240 & $-\cdots$ & 5240 & 480 & 190 & 3846 \\
\hline 20 & 4.0 & 4.0 & - & 8531 & 7364 & 1630 & $\cdots$ & 1630 & $\therefore$ & 130 & 3846 \\
\hline 21 & 11.0 & 9.6 & -- & 18233 & 17276 & 4800 & "---- & 4800 & $\because-$ & 130 & 3846. \\
\hline 22 & 9.5 & 9.5 & 5.3 & 15101 & 13970 & 3900 & $\cdots$ & 3900 & 864 & 260 & 3054 \\
\hline 23 & 4.0 & 4.0 & --- & 5616 & 3784 & 20 & ---- & 20 & -- & 70 & 3054 \\
\hline 24 & 6.1 & 6.1 & -- & 11410 & 9341 & 1400 & 8906 & 10306 & -- & 110 & 3054 \\
\hline 25 & 11.1 & 11.1 & 5.3 & 18359 & 17155 & 6440 & $\cdots-$ & 6440 & 800 & 260 & 3054 \\
\hline 26 & 10.7 & 10.7 & 1.7 & 16371 & 14936 & 4560 & $\cdots$ & 4560 & 320 & 240 & 3054 \\
\hline 27 & 6.5 & 6.5 & -- & 7353 & 4812 & 710 & $\cdots$ & 710 & $-\infty$ & 90 & 3054 \\
\hline 28 & 7.5 & 7.5 & 6.3 & 16538 & 15032 & 4710 & $-\cdots$ & 4710 & 928 & 190 & 3054 \\
\hline 29 & 3.8 & 3.8 & -- & 9503 & 9007 & 890 & ---- & 890 & --- & 80 & 3054 \\
\hline $\begin{array}{l}30 \\
31\end{array}$ & 5.2 & 5.2 & --- & 4603 & 3683 & 110 & $\cdots-$ & 110 &.-- & 90 & 3054 \\
\hline TUTAI. & 270.4 & 252.0 & $70 . ?$ & 450529 & 397381 & 111867 & 19326 & 131193 & 12480 & 5030 & $101840^{8}$ \\
\hline
\end{tabular}


1:0 kWe S.JLAR PRO.JECT

MONTHLY ENERGY BALANIE

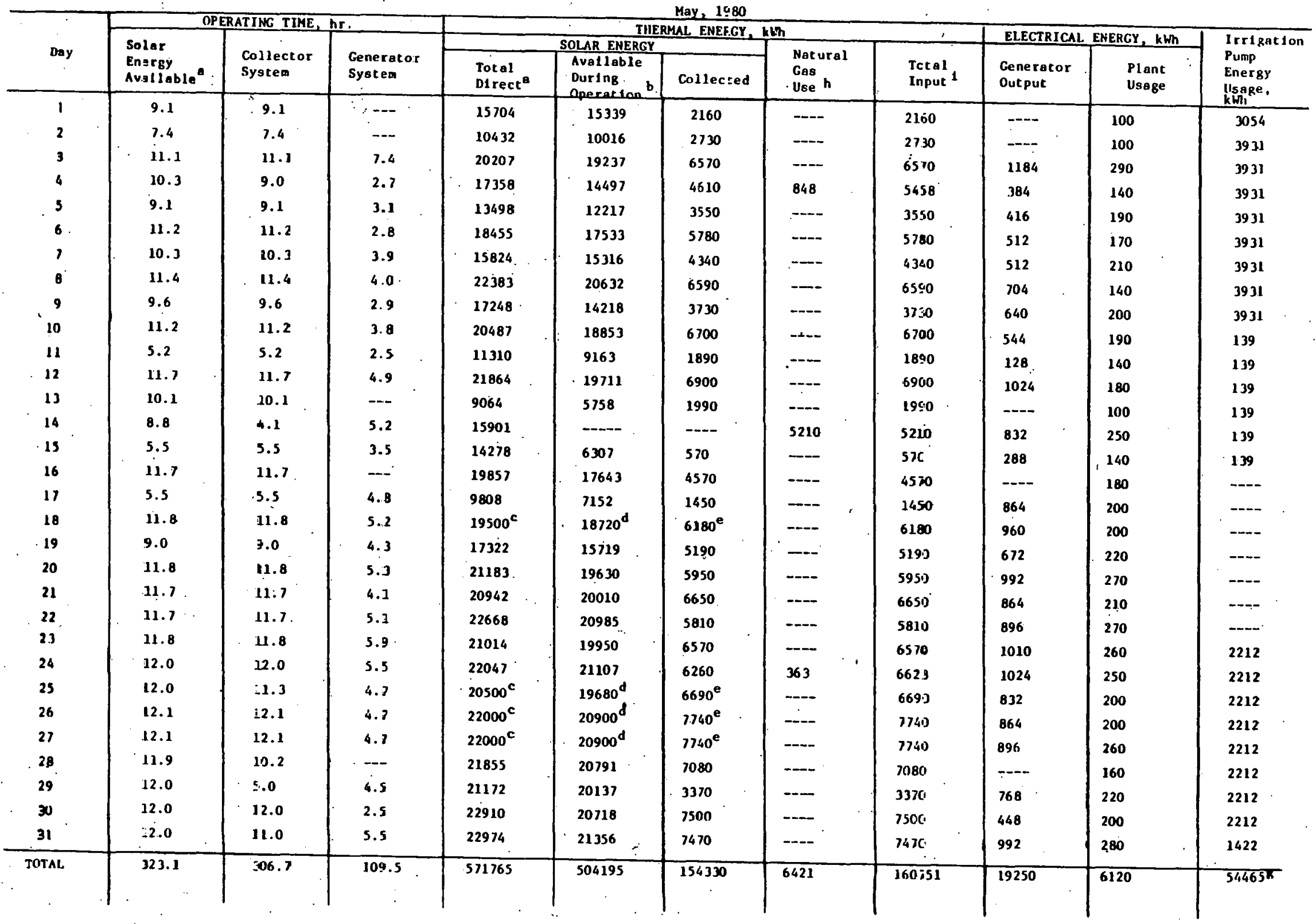

Note: See page 32 for footnotes. 
150 KLIE SOLAR PROJECT

MONTHLY ENERGY BNAANCE

\begin{tabular}{|c|c|c|c|c|c|c|c|c|c|c|c|}
\hline \multirow{3}{*}{ Day } & \multicolumn{3}{|c|}{ OPERATING TIME, hr. } & \multirow{2}{*}{\multicolumn{5}{|c|}{$\begin{array}{l}\text { THERMAL ENERGY, thh } \\
\text { SOLAR ENERGY }\end{array}$}} & & & \\
\hline & \multirow{2}{*}{\begin{tabular}{|l|} 
Solar \\
Encigy \\
Avbl lable
\end{tabular}} & \multirow[b]{2}{*}{$\begin{array}{l}\text { Collector. } \\
\text { System }\end{array}$} & \multirow[b]{2}{*}{$\begin{array}{l}\text { Gene rator } \\
\text { Systen }\end{array}$} & & & & & & \multicolumn{2}{|c|}{ ELECTRICAL ENERCY, $\mathrm{kHh}$} & \multirow{2}{*}{$\begin{array}{l}\text { Irrigeclon } \\
\text { Pump } \\
\text { Energy } \\
\text { Usgase. } \\
\text { kwh }\end{array}$} \\
\hline & & & & $\begin{array}{l}\text { Total } \\
\text { DI rect a }\end{array}$ & $\begin{array}{l}\text { Avallable } \\
\text { During } \\
\text { Operacion b }\end{array}$ & Collected & $\begin{array}{l}\text { Natural } \\
\text { Gas } \\
\text { Use } \\
\end{array}$ & $\begin{array}{l}\text { Total } \\
\text { Input } 1\end{array}$ & $\begin{array}{l}\text { Generator } \\
\text { Output }\end{array}$ & $\begin{array}{l}\text { Plailt } \\
\text { Usage }\end{array}$ & \\
\hline 1 & 12.2 & 11.0 & 7.0 & 22273 & 21101 & 6830 & ---- & 6830 & 1100 & 300 & 1422 \\
\hline 2 & 12.2 & 12.2 & 5.1 & 20900 & 19381 & 7670 & $\cdots-$ & $\begin{array}{c}7670 \\
:=\end{array}$ & 1000 & 210 & 1422 \\
\hline 3 & 12.0 & 11.0 & 4.8 & 22427 & 20896 & 6660 & $\therefore$ & 6660 & 900 & 240 & 1422 \\
\hline 4 & 11.3 & 10.5 & 5.2 & 21684 & 19520 . & 7640 & $\cdots$ & $7640^{\circ}$ & 1010 & 250 & 1422 \\
\hline 5 & 7.0 & 12.0 & 1.0 & 16563 & 15058 & 5320 &.+- & 5320 & 180 & 200 & 1422 \\
\hline 6 & 12.2 & 12.2 & 3.4 & 17280 & 16303 & 7260 & $\cdots$ & 7260 & 630 & 280 & 1422 \\
\hline 7 & 11.0 & 11.0 & 7.0 & 16477 & 15305 & 5850 & --.-- & $5850^{\circ}$ & 1290 & 220 & 1422 \\
\hline B & 5.5 & 5.5 & --- & 9839 & 8163 & 1950 & ----- & 1950 & --- & 90 & 1422 \\
\hline 9 & 11.0 & 11.0 & 3.4 & 16770 & 15078 & 6330 & ---- & 6330 & 640 & 210 & 1422 \\
\hline 10 & 12.3 & 7.0. & 3.? & 21.325 & 16380 & 4400 & ---- & 4400 & 700 & 260 & 1422. \\
\hline 11 & 12.0 & 8.3 & -- & 22020 & 20951 & 5268 & $-\cdots$ & 5268 & ---- & 200 & 808 \\
\hline 12 & $12: 3$ & 8.5 & 4.2 & 21131 & 14911 & 6009 & ---- & 6009 & 830 & 280 & 808 \\
\hline 13 & 12.1 & 11.9 & 8.9 & 21933 & 20206 & 1990 & --.- & 1990. & 1740 & 320 & 808 \\
\hline 14 & 11.2 & 1.1 .2 & 3.6 & 17707 & 16941 & 5130 & ----亡 & 5130 & 530 & 200 & 808 \\
\hline 15 & 12.1 & 12.1 & 4.2 & 22630 & 21694 & 7150 & $-\cdots$ & 7150 & 990 & 270 & 808 \\
\hline 16 & 12.0 & 12.0 & 6.5 & 19622 & 18175 & 7410 & -..- & 7410 & 1240 & 330 & 808 \\
\hline 17 & 12.0 & 12.0 & 5.1 & 18938 & 17742 & 5770 & --.-- & 5770 & 830 & 200 & 808 \\
\hline 18 & 12.0 & 12.0 & 4.6 & 17280 & 16180 & 5935 & $\cdots--$ & 5935 & 660 & 380 & 808 \\
\hline 19 & 9.9 . & 7.5 & 3.6 & 18273 & 15005 & 3393 & 2787 & 6180 & 240 & $240^{\circ}$ & 3673 \\
\hline 20 & 12.1 & 11.8 & 5.9 & 18516 & 17430 & 5718 & ---- & 5718 & 990 & 290 & 3673 \\
\hline 21 & 11.9 & 11.9 & 4.8 & 19927 & 18900 & 5912 & ----- & 5912 & 700 & 270 & 3673 \\
\hline 22 & 12.3 & 10.5 & $\cdots$ & 18417 & $15694^{\circ}$ & 2847 & --.- & 2847 & ---- & 260 & 3673 \\
\hline 2.3 & 12.5 & 12.5 & 10.7 & 21452 & 20604 & 6357 & --- & 6357 & 1140 & 320 & 3673 \\
\hline 24 & 12.3 & $6.4 \therefore$ & 1.9 & 21552 & 20426: & 7106 & - & 7106 & 290 & $320^{\circ}$ & 3673 \\
\hline 25 & 12.0 & 12.0 & 5.6 & 21453 & 20020 & 7510 & --.- & 7510 & 1180 & 280 & 3673 \\
\hline 26 & 11.5 & 11.5 & 4.5 & 19916 & $19151^{\circ}$ & 6534 & ---- & 6534 & 940 & 220 & 2569 \\
\hline 27 & 12.0 & 12.0 & 1.0 & 17398 & 11375 & 2614 & --- & 2614 & $250^{\circ}$ & 250 & 2569 \\
\hline 28 & 11.9 & 11.9 & 3.3 & 14540 & 13255 & 4471 & ---- & 4471 & 520 & 250 & 2569 \\
\hline 29. & 11.2 & .11 .1 & 5.6 & $14000^{c}$ & $13200^{d}$ & $4620^{\mathrm{e}}$ & -.... & 4620 & 1030 & 280 & 2569. \\
\hline $\begin{array}{l}30 \\
31\end{array}$ & 10.0 & 9.8 & 3.5 & 15155 & 14602 & 5370 & ----- & 5370 & 450 & 220 & 2569 \\
\hline TOTAL. & 348.2 & 323.3. & 128.7 & 567198 & 513649 & 167024 & 2787 & 169811 & 22000 & 7640 & 59240 \\
\hline
\end{tabular}

Ncte: See page 32 for footnotes. 
190 kWO SOIAR PROJECT

MONTILT ENERCY BAIANCE

\begin{tabular}{|c|c|c|c|c|c|c|c|c|c|c|c|}
\hline \multirow{3}{*}{ Day } & \multicolumn{3}{|c|}{ DPERATIHG TIME, hr. } & \multirow{2}{*}{\multicolumn{5}{|c|}{$\begin{array}{l}\text { THERMAL ENERCY, } \mathrm{kWh} \\
\text { SOL.AR ENERCY }\end{array}$}} & & & \\
\hline & \multirow{2}{*}{$\begin{array}{l}\text { Selaz } \\
\text { Energy } \\
\text { Avellable }\end{array}$} & \multirow[b]{2}{*}{$\begin{array}{l}\text { Coilector } \\
\text { Syatew }\end{array}$} & \multirow[b]{2}{*}{$\begin{array}{l}\text { Cenerator } \\
\text { Syotem }\end{array}$} & & & & & & \multicolumn{2}{|c|}{ EI.ECTRICAI. ENERGY, kWll } & \multirow{2}{*}{$\begin{array}{l}\text { Irrigotion' } \\
\text { Pump } \\
\text { Energy } \\
\text { Ugagge. } \\
\text { kWh }\end{array}$} \\
\hline & & & & $\begin{array}{l}\text { Jotal } \\
\text { D1 rect a }\end{array}$ & $\begin{array}{l}\text { Avallable } \\
\text { During } \\
\text { Ouferactan }\end{array}$ & collected & $\begin{array}{l}\text { Natural } \\
\text { Gass } \\
\text { Use h }\end{array}$ & $\begin{array}{l}\text { Tctal } \\
\text { Irput }\end{array}$ & $\begin{array}{l}\text { Cenerator } \\
\text { Output }\end{array}$ & $\begin{array}{l}\text { Plant } \\
\text { Usage }\end{array}$ & \\
\hline 1 & 11.0 & 10.3 & 2.0 & $14011^{\circ}$ & 11707 & 1453 & $\cdots-$ & .1453 & 135 & 250 & 2569 \\
\hline 2 & 12.4 & 12.4 & 4.5 & 20223 & 19694 & 6923 & $\cdots$ & 6923 & 900 & 270 & 2569 \\
\hline 3 & 12.4 & 10.7 & 7.9 & 23877 & 21155 & 3060 & ----- & $j 060$ & 1350 & 380 & 2990 \\
\hline 4 & 12.0 & 12.0 & 9.2 & 24543 & 22509 & 8393 & ---- & 8.193 & 1240 & 330 & 2990 . \\
\hline 5 & 11.9 & 11.9 & 5.7 & 20498 & 20131 & 6922. & --- & $6 \$ 22$ & 1050 & 280 & 2990 \\
\hline 6 & 1.5 & $m$ & -- & 4872 & --- & $\cdots$ & $\cdots$ & ---- & --- & 110 & 3853 \\
\hline 7 & 7.5 & 7.5 & 3.8 & 17738 & 15246 & 4980 & ----- & 4980 & 690 & 230 & 3853 \\
\hline B & 12.0 & 12.0 & 6.2 & 22357 & 20748 & 7328 & $\therefore$. & 7328 & 1080 & 310 & 3853 \\
\hline 9 & 5.6 & 5.3 & 2.6 & 17118 & 4564 & 1283 & 1999 & 3982 & 330 & 170 & 3853 \\
\hline 10 & 6.8 & 6.0 & 1.6 & 5994 & 4045 & $\cdot 1371$ & $\therefore--$ & 1371 & 280 & 190 & 3853 \\
\hline 11 & 12.0 & 10.5 & 4.1 & 20908 & 19752 & 5857 & $-\cdots$ & 5.857 & 810 & 270 & 3853 \\
\hline 12 & 6.0 & -6.0 & 3.0 & 10721 & 8858 & 2734 & $\cdots$ & 2734 & 400 & 200 & 3853 \\
\hline 13 & 10.0 & 10.0 & 5.1 & 17575 & 16964 & 5772 & $\cdots$ & 5772 & 820 & 230 & 3853 \\
\hline 14 & 11.0 & 10.0 & 5.0 & 18908 & 17910 & 6002 & ----- & 6002 & 950 & 280 & 3853 \\
\hline 15 & 11.9 & 11.9 & 5.8 & 19860 & 18526 & 5978 & $-\because--$ & 5978 & .910 & $270^{\circ}$. & 3021 \\
\hline 16 & 9.0 & 9.D & 4.7 & 18398 & 16732 & 5089 & ---- & 5089 & $? 30$ & 250 & 3021 \\
\hline 11 & 11.9 & 11.8 & 4.1 & 18879 & 17560 & 4506 & $\cdots$ & 4506 & $700^{\circ}$ & 260 & 3021 \\
\hline 18 & 11.4 & 11.4 & 4.1 & 17398 & 15972 & 4782 & ----- & 7782 & 660 & 250 & 3021 \\
\hline 19 & 3.5 & 3.5 & 1.3 & 5822 & 4475 & 194 & 2423 & 2627 & 160 & 180 & 3021 \\
\hline 20 & 8.8 & 8.7 & 3.7 & 15166 & 13481 & 4316 & $\cdots-$ & .4316 & 620 & 200 & 3021 \\
\hline 21 & 10.7. & 10.7 & 4.6 & 17395 & 16476 & 5418 & $\therefore . .$. & 5418 & 290 & 260 & 3366 \\
\hline 22 & 4.3 & 4.3 & -- & 7501 & 6456: & 2132 & ----- & 1132 & -- & 130 & 3366 \\
\hline 2.1 & 8.2 & 8.2 & 5.3 & 4789 & 3512 & 1169 & $m$ & 1149 & 840 & 270 & 3366 \\
\hline 24 & 5.0 & 5.0 & 1.9 & 9453 & 8757 & 2759 & $\cdots$ & 2759 & 300 & $2 i 0$ & 3366 \\
\hline 25 & 11.5 & 11.5 & 3.6 & 17147 & 16161 & 5451 & $-\cdots$ & 5451 & 670 & 220 & 3366 \\
\hline 26 & 11.5 & 11.5. & 5.1. & 13572 & 12493 & 4562 & $\cdots$ & 4562 & 970 & $320^{\circ}$ & 3366 \\
\hline 27 & 11.1 & 11.1 & 3.6 & 17978 & 16442 & 5899 & $\cdots$ & 5899 & 620 & 280 & 3366 . \\
\hline 28 & 9.2 . & 9.2 & 5.1 & 14604 & 13730 & 4920 & --- & 4920 & 1040. & 330 & 3366 \\
\hline 29 & 5.5 & 5.5 & 1.5 & 9919 & 8161 & 2501 & $-\infty$ & 2501 & 220 & 230 & 3366 \\
\hline 30. & 10.9 & 10.9 & 4.3 & 12007 & 11049 & 4105 & $m$ & .4105 & 700 & 250 & 3366 \\
\hline $3 i$. & 11.2 & 11.2 & 5.1 & 19172 & 18007 & 6160 & $\cdots$ & 6160 & 930 & 310 & 3366. \\
\hline Totai. & $\begin{array}{l}287.7 \\
:\end{array}$ & 279.9 & 124.5 & 478481 & 421273 & 137999 & 4422 & 140421 & 2089.5 & 7720 & $103937^{8}$ \\
\hline
\end{tabular}

Note: See page 32 for fcotnotes. 
150 KW SOIAR PROUECT

MONTHLY ENERCY BALANC:

\begin{tabular}{|c|c|c|c|c|c|c|c|c|c|c|c|}
\hline \multirow{3}{*}{ Day } & \multicolumn{3}{|c|}{ OPERATING TIME, hr. } & \multirow{2}{*}{\multicolumn{5}{|c|}{$\begin{array}{c}\text { THERMAL ENERGY, klh } \\
\text { SOLAR ENERGY }\end{array}$}} & & & \\
\hline & \multirow{2}{*}{$\begin{array}{l}\text { Solar } \\
\text { Energy } \\
\text { Avallable }\end{array}$} & \multirow[b]{2}{*}{$\begin{array}{l}\text { Collector } \\
\text { Systee }\end{array}$} & \multirow[b]{2}{*}{$\begin{array}{l}\text { Cenerator } \\
\text { System }\end{array}$} & & & & & & \multicolumn{2}{|c|}{ ELECTRICAL DHERCY, kLm } & \multirow{2}{*}{$\begin{array}{l}\text { Irritgat Ion } \\
\text { Punp } \\
\text { Energy } \\
\text { Ugege. } \\
\text { kunge. }\end{array}$} \\
\hline & & & & $\begin{array}{l}\text { Total } \\
\text { D1rect }\end{array}$ & $\begin{array}{l}\text { Avallable } \\
\text { Durlng } \\
\text { Operacion b }\end{array}$ & Collected & $\begin{array}{l}\text { Natural } \\
\text { Gas } \\
\text { Useh } \\
\end{array}$ & $\begin{array}{l}\text { Total } \\
\text { Input } 1\end{array}$ & $\begin{array}{l}\text { Tenerator } \\
\text { Output }\end{array}$ & $\begin{array}{l}\text { Plant } \\
\text { Usage }\end{array}$ & \\
\hline 1 & $11 i^{5}$ & 11.5 & 4.5 & 17412 & 16926 & 5761 & --- & 5761 & 700 & 300 & 3366 \\
\hline 2 & 11.0 & 11.0 & 3.4 & 15611 & 14960 & 5144 & $-\cdots$ & 5144 & 590 & 160 & 3366 \\
\hline 3 & 11.6 & 11.6 & 3.0 & 17994 & 17461 & 5657 & $-\cdots$ & 5657 & 500 & 220 & 3366 \\
\hline 4 & 7.3 & 7.3 & 2.9 & 14601. & 113100 & 3904 & --- & 3365 & 580 & 240 & 3366 \\
\hline 5 . & 10.6 & 10.6 & 5.1 & 16686 & 15547 & 4769 & $-\dot{-}$ & 3767 & 980 & 280 & 3366 \\
\hline 6 & 11.1 & 11.1 . & 4.7 & 19234 & 17785 & 5722 & $-\cdots$ & 4392 & 680 & 240 & 3366 \\
\hline 7 & 11.1 & 11.1 & 4.5 & 20133 & 18535 & 5836 & --- & 4683 & 670 & 290 & 336.6 \\
\hline 8 . & 6.0 & 6.0 & 2.8 & 11569 & 10316 & 2713 & $\because$ & 2055 & 460 & $240^{\circ}$ & 3366 \\
\hline 9 & 5.5 & 5.5 & 1.1 & 8378 & 6426 & 1564 & $\cdots$ & 955 & 150 & 170 & 3492 \\
\hline 10 & 5.5 & 5.5 & 1.8 & 8926 & 7567. & 2098 & --- & 1369 & 270 & 200 & 3492 \\
\hline 11 & 9.5 & 9.5 & 3.3 & 11275 & 9219 & 2548 & ---- & 1071 & 500 & 260 & 3492 \\
\hline 12 & 9.2 & 9.2 & 2.8 & 12987 & $1217 ?$ & 3002 & -.- & 2099 & 410 & 230 & 3492 \\
\hline 13 & 7.5 & 7.5 & 3.1 & 7815 & 7033 & 1784 & 1860 & 3644 & 440 & 240 & 3492 \\
\hline 14 & 3.4 & 3.4 & -- & 8707 & 4702 & 973 & ---- & 973 & --- & 100 & 3492 \\
\hline 15 & 10.0 & 10.0 & 4.6 & 18073 & 16301 & 4360 & $\cdots$ & 4360 & 670 & 250 & 3492 \\
\hline 16 & 11.0 & 11.0 & 4.6 & 22792 & 20105 & 6313 & --- & 6313 & 680 & 260 & 3311 \\
\hline 17 & 11.1 & 11.1 & 6.8 & 22418 & 20233 & 6586 & -- & 6586 & 1190 & 310 & 3311 \\
\hline 18 & 6.0 & 6.0 & 2.9 & 13969 & 12220 & 3150 & ---- & 3150 & 430 & 180 & 331.1 \\
\hline 19 & 8.5 & 8.5 & 4.6 & 19080 & 17725 & 4602 & - & 4602 & 680 & 250 & 3311 \\
\hline 20 & 11.1 & 11.1 & 3.8 & 20806 & 19394 & 5373 & ---- & 5373 & 630 & 250 & 3311 \\
\hline 21 & 11.1 & 11.1 & 6.2 & 20645 & 18911 & 5670 &.-- & 5670 & 1000 & 280 & 3311 \\
\hline 22 & 11.0 & 11.0 & 3.2 & 8415 & 5890 & 2866 & $\cdots$ & 2866 & 520 & 250 & 3311 \\
\hline 23 & 0 & 0 & $\cdots$ & 6938 & --- & ---- & $-\cdots$ & - & ---- & 200 & 3311 \\
\hline 24 & 3.9 & 3.9 & -- & 8730 & 4929 & 1120 & ---- & 1120 & $-\cdots$ & 120 & 3311 \\
\hline 25 & 9.5 & 9.5 & 5.1 & 16601 & 16011 & 4732 & $\cdots$ & 4732 & 800 & 190 & 3311 \\
\hline 26 & 10.8 & 10.8 & 4.7 & 17207 & 16661 & 4982 & ---- & 4982 & 790 & 280 & 3500 . \\
\hline 27 & 10.7 & 10.7. & 4.4 & 17220 & 16909 & 5088 & -- & 5088 & 810 & 230 & 3500 \\
\hline 28 & 9.9 & 9.9 & 4.7 & 15910 & 14030 & 3604 & 1674 & 5278 & 720 & 270 & 3500 \\
\hline 29 & 10.2 & 10.2 & 3.7 & .16306 & 15232 & 4419 & $\cdots$ & 4419 & 580 & 210 & 3500 \\
\hline 30 & 10.7 & 10.7 & 3.2 & 19830 & 18427 & 5334 & --- & 5334 & 630 & 220 & 3500 \\
\hline 31 & 10.8 & 10.8 & 5.3 & 19921 & 18511 & 5590 & --- & 5590 & 1000 & 270 & 3500 \\
\hline TотAl. & 277.1 & 277.1 & 110.3 & 476243 & 423243 & 125264 & 3534 & 128798 & 18530 & 7190 . & $105482^{8}$ \\
\hline Note: & page 32 & footno & & & & & & & & & \\
\hline
\end{tabular}


Notations on the monthly energy balance summaries refer to the following definitions and assumptions used in the compilation of energy budget information:

a. Direct normal radiation when $>300 \mathrm{~W} / \mathrm{m}^{2}$ (>945.7 Btu/ft.h).

b. Available solar energy during collector system operation.

c. Data unavailable; estimate based on hours of operation and seasonal data.

d. Data unavailable; estimate based on seasonal ratio and operating time.

e. Data unavailable; estimate based on seasonal efficiency and operating time.

f. Measured periodically; apportionment based on daily, equipment usage.

g. Measured periodically; apportioned equally to each day.

h. Natural gas used to heat Caloria. Boiler efficiency was assumed to be 1008 .

i. Collected solar energy plus natural gas usage. Since boiler

- efficiency was assumed to be 100\%, energy gain by Caloria is overestimated. 


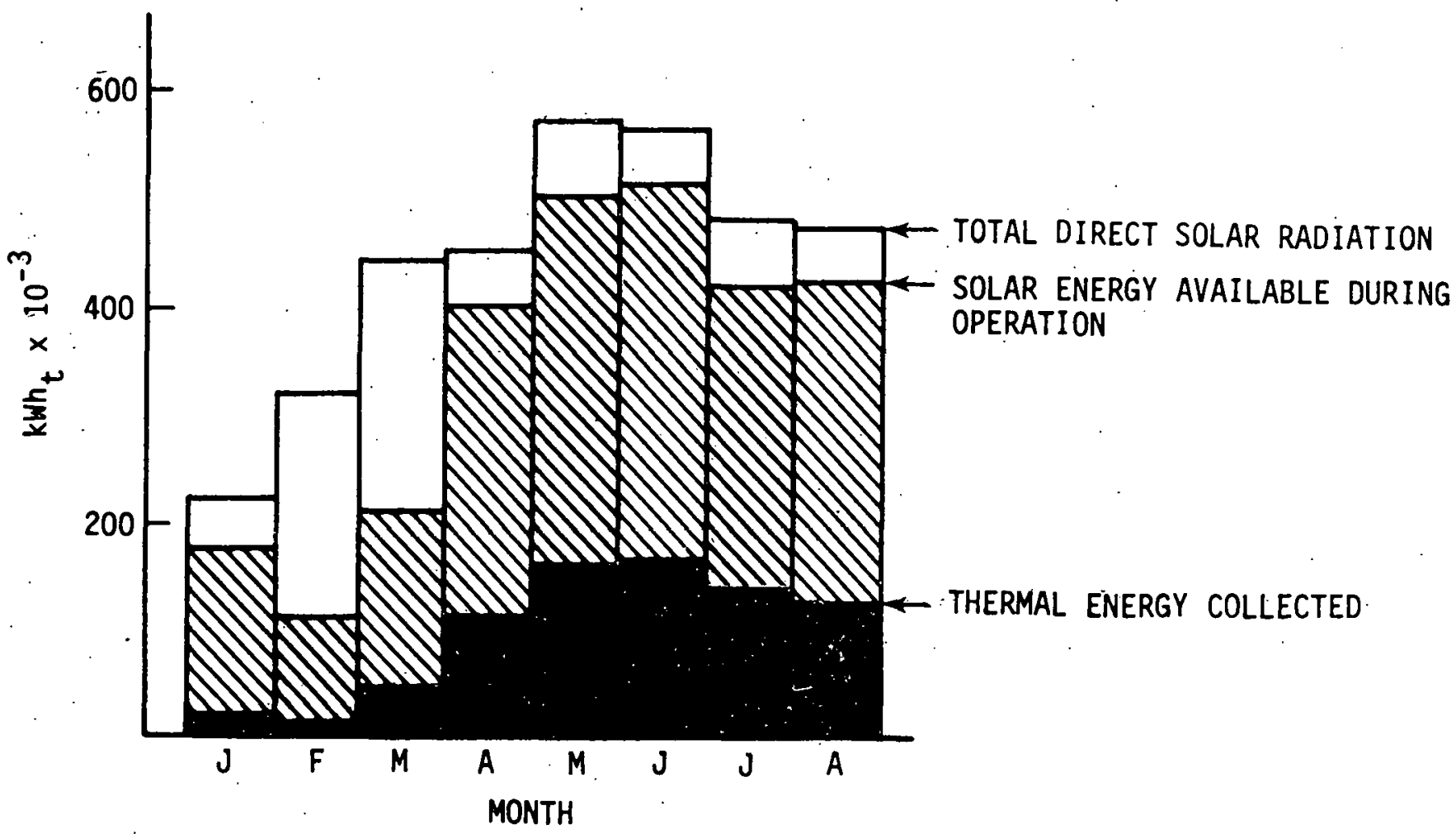

Figure 1. Solar Energy Available and Thermal Energy Collected by the Plant from January through August 1980. Low amounts of available insolation during operation in February and March are due to pump outage for repair.

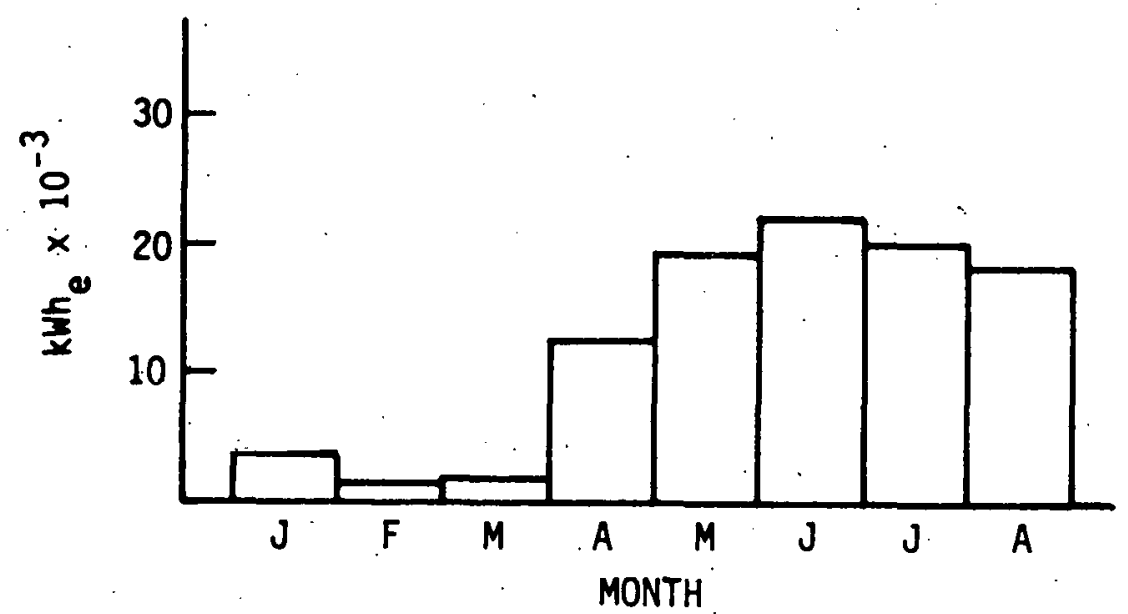

Figure 2. Electrical Energy Generated by the Plant from January through August 1980 


\author{
PERFORMANCE OF THE SOLAR COLLECTOR SUBSYSTEM \\ AT THE \\ COOLIDGE SOLAR IRRIGATION FACILITY \\ ON \\ 17, 18, AND 24 DECEMBER 1979
}

\title{
Introduction
}

Collector system operational data were: obtained on 17,18 , and 24 December 1979 to determine performance near winter solstice. This report describes the test procedures and presents the test data.

\section{Procedures}

Collectors were rinsed with untreated water and air dried prior to the 17 December test. Some water spots remained on the reflector surface: Receiver cover tubes also were rinsed externally, but most tube interiors had some dust accumulation.

Oil in storage was preheated to approximately $200^{\circ} \mathrm{C}\left(392^{\circ} \mathrm{F}\right)$ on days prior to the test. Morning test operation began with minimal, 560 to $850 \mathrm{l} / \mathrm{min}(20$ to $30 \mathrm{gal} / \mathrm{min})$, oil flow rates and with oil recirculation from the buffer tank only. The flow rate was adjusted to maintain the desired constant collector system output temperature.

When buffer tank oil temperatures reached a preset value, the primary oil storage tank was included in the flow loop. If primary tank oil temperatures were significantly lower than buffer tank oil temperatures, inclusion resulted in a dramatic change in test conditions and collector performance. In some cases, collector outlet temperatures were reduced sufficiently to cause a return to the buffer tank oil recirculation mode. 
Seventeen and 18 December were mostly cloudless, but 24 December had intermittent high cloudiness. Collector outlet temperature goals were $288^{\circ}, 232^{\circ}$, and $260^{\circ} \mathrm{C}\left(550^{\circ}, 450^{\circ}\right.$, and $\left.500^{\circ} \mathrm{F}\right)$. Sustained operation at the desired outlet temperature was obtained only with the $232^{\circ} \mathrm{C}\left(450^{\circ} \mathrm{F}\right)$ test on 18 December.

\section{$\underline{\text { Results }}$}

On 17 December, the $1.3-\ell / \mathrm{s}(20-\mathrm{gal} / \mathrm{min})$ flow rate and buffer tank oil recirculation were maintained until midafternoon to attain the desired $288^{\circ} \mathrm{C}\left(550^{\circ} \mathrm{F}\right)$ collector system output temperature. 'linis temperature was maintained only briefly. The inclusion of the main storage tank in the flow loop again reduced the outlet temperature. Collector efficiency was less than $10 \%$ most of the day, averaging 4.4\%. The brief spike in the efficiency curve is an erroneous indication due to low inlet temperatures at the moment of tank switching while outlet temperatures were still high.

The 18 December test resulted in almost constant collector output at $232^{\circ} \mathrm{C}\left(450^{\circ} \mathrm{F}\right)$. The efficiency curve is U-shaped. Collector efficiency was $8.0 \%$ to $8.4 \%$ near noon, nearly $20 \%$ for a short periud in midmorning, and about $30 \%$ for a moment in midafternoon. Average daily collector efficiency was 9.98 .

'Ihe data of 24 Vecember show performance on a day of interilltent cloudiness and less direct insolation. Buffer-tank-only flow recirculation occurred, and collector outlet temperatures gradually increased to over $200^{\circ} \mathrm{C}\left(392^{\circ} \mathrm{F}\right)$. Midafternoon insolation improved, and efficiency increased from about $5 \%$ to more than 10\%. Operation on this cloudy day was productive, but collection efficiency averaged only 3.78 .

\section{Conclusions}

Collector system efficiency ranged from about $8 \%$ at noon to nearly $20 \%$ at midmorning and midafternoon with $200^{\circ} \mathrm{C}\left(392^{\circ} \mathrm{F}\right)$ inlet and $232^{\circ} \mathrm{C}\left(450^{\circ} \mathrm{F}\right)$ outlet temperatures on 18 December. The north-south 
orientation of the collectors dictates that the collection efficiency shall peak in midmorning and midafternoon and dip at noon. This pattern occurs because the sun is more normal to the collectors in midmorning and midafternoon than at noon. The desired outlet temperature of $288^{\circ} \mathrm{C}\left(550^{\circ} \mathrm{F}\right)$ was obtained for only a short time on 17 December. Tests on 24 December yielded cloudy-weather performance data.

Collection efficiencies are low near winter solstice. Little high-temperature collection can be attained by the subsystem. The subsystem performance could be improved by changing the collector reflector material from Coilzak to a high-reflectivity material such as FEK-244. Power plant operation with solar energy can be maintained throughout the winter season through the use of a fossil fuel heater plumbed in series with the collector field. In this mode, the collector field would be used to preheat the Caloria for the fossil fuel heater.

Collection efficiency is defined as the ratio of thermal energy. gained by the $o i l$ as it passes through the collector field ( $\dot{m} C_{p} \Delta T$ ) to the direct normal insolation measurement multiplied by the collector aperture area. Oil properties are

$$
\begin{array}{lll}
\text { Density }=55.06-0.02337 \mathrm{~T} & \text { (Density in } \left.1 \mathrm{~b} / \mathrm{ft}^{3} ; \mathrm{T} \text { in }{ }^{\circ} \mathrm{F}\right) \\
C_{p}=0.4458+4.796 \times 10^{-4} \mathrm{~T} & \left(\mathrm{C}_{\mathrm{p}} \text { in } \mathrm{Btu} / 1 \mathrm{~b} \cdot{ }^{\circ} \mathrm{F} ;\right. & \left.\mathrm{T} \text { in }{ }^{\circ} \mathrm{F}\right)
\end{array}
$$




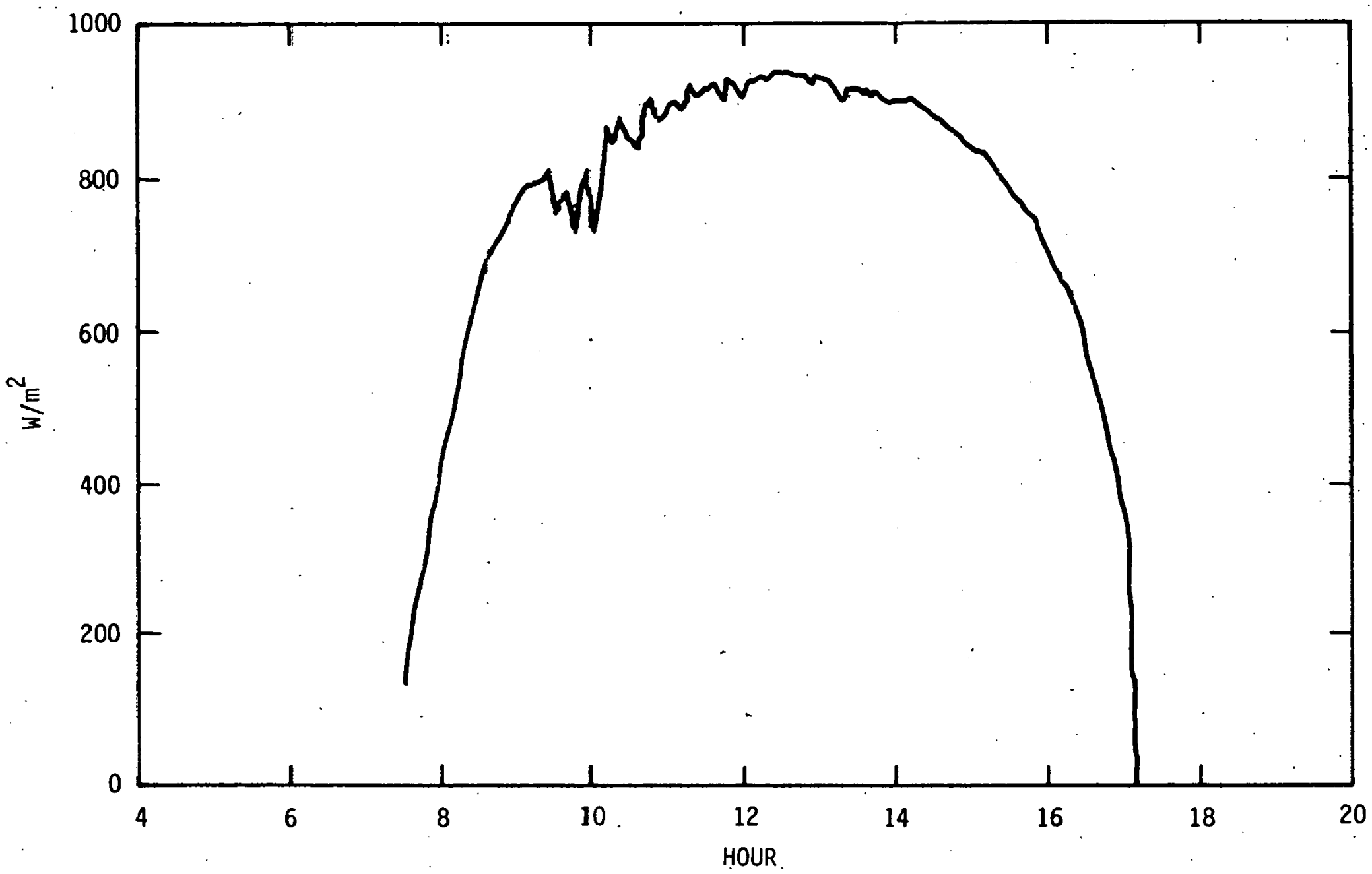

Figure 1. Insolation, 351-1979 


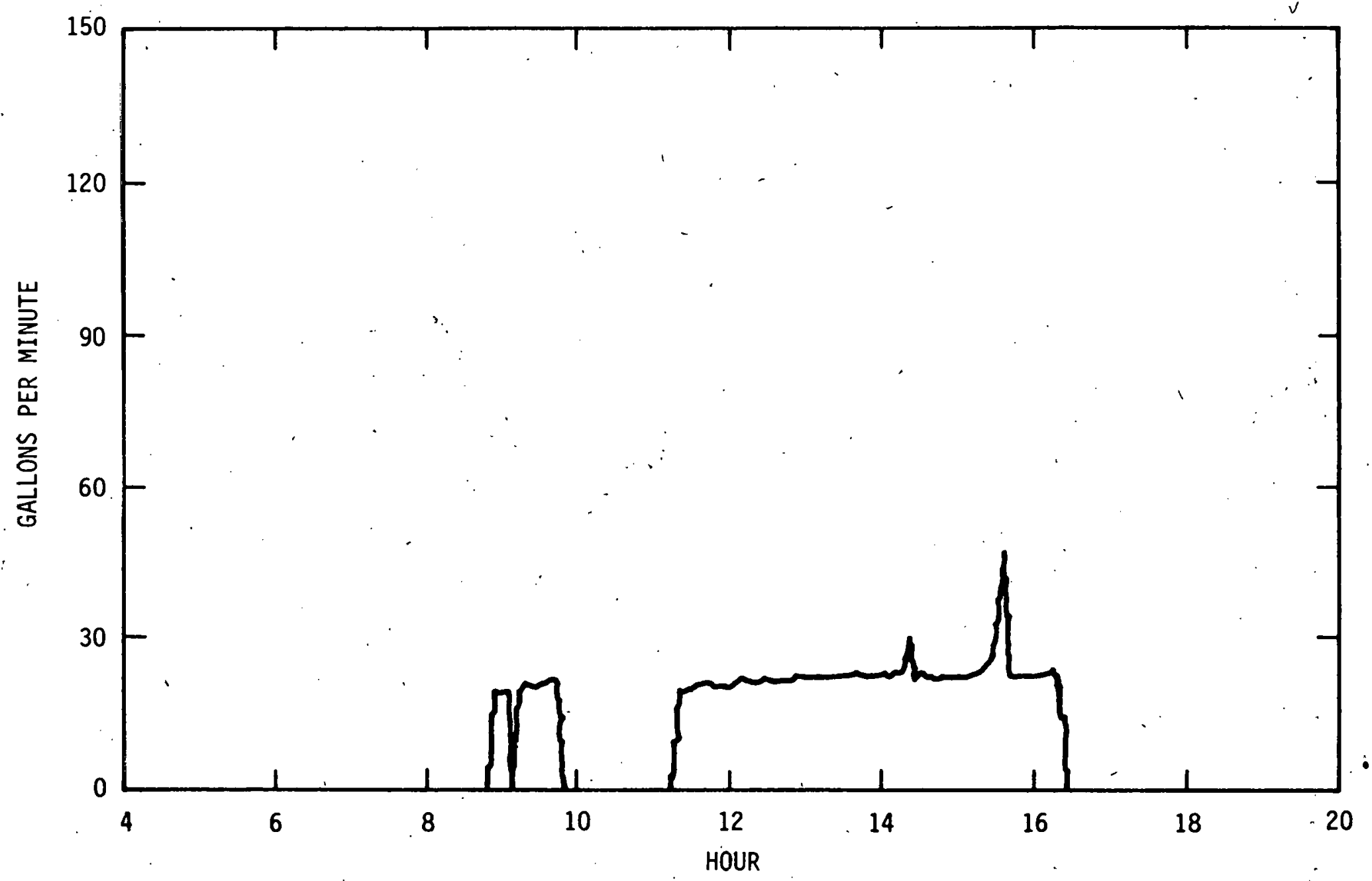

Figure 2. Flow Rate, 351-1979 
D

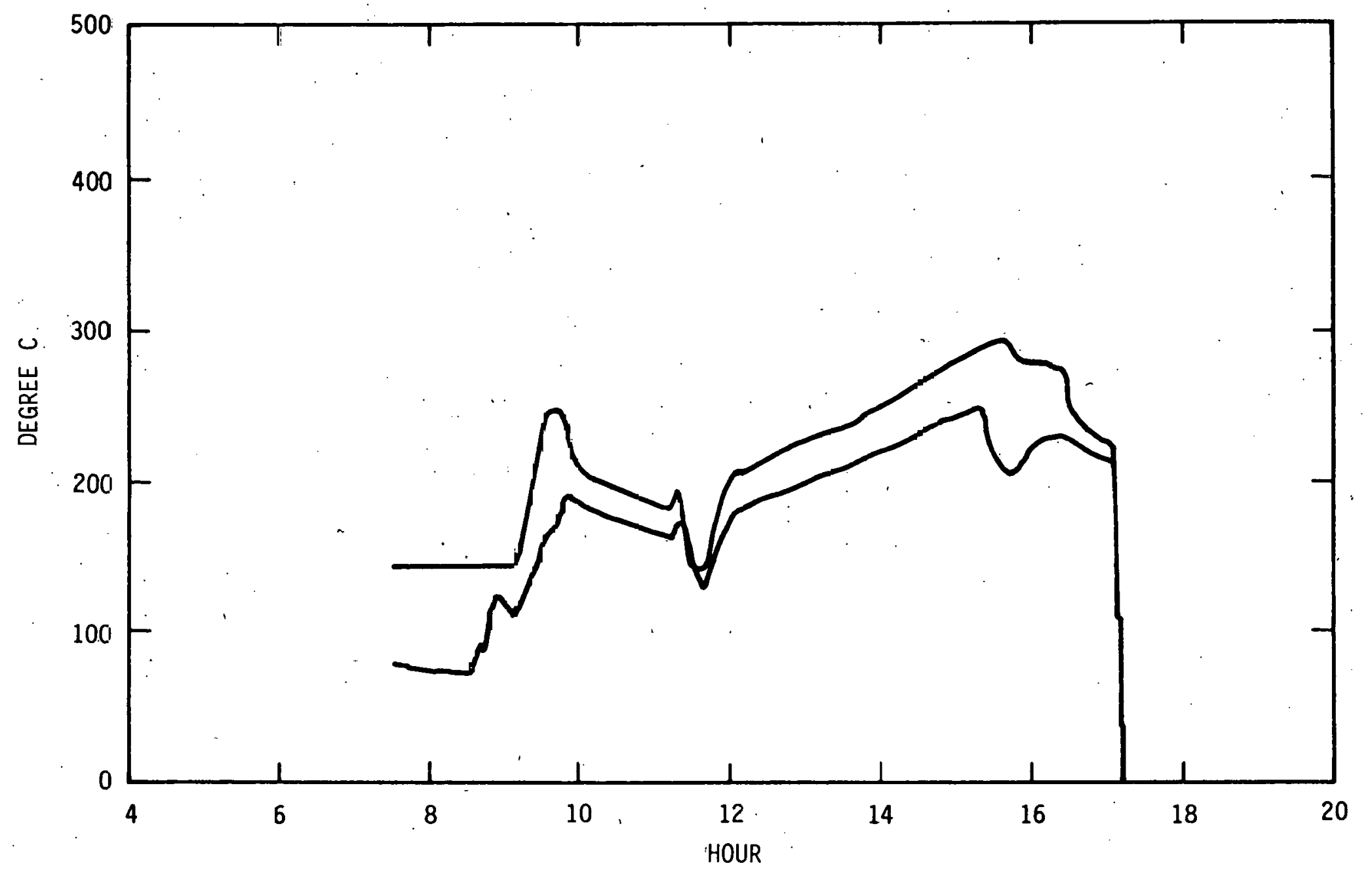

Figure 3. Ir let and Outlet Temperatures, 351-1979 


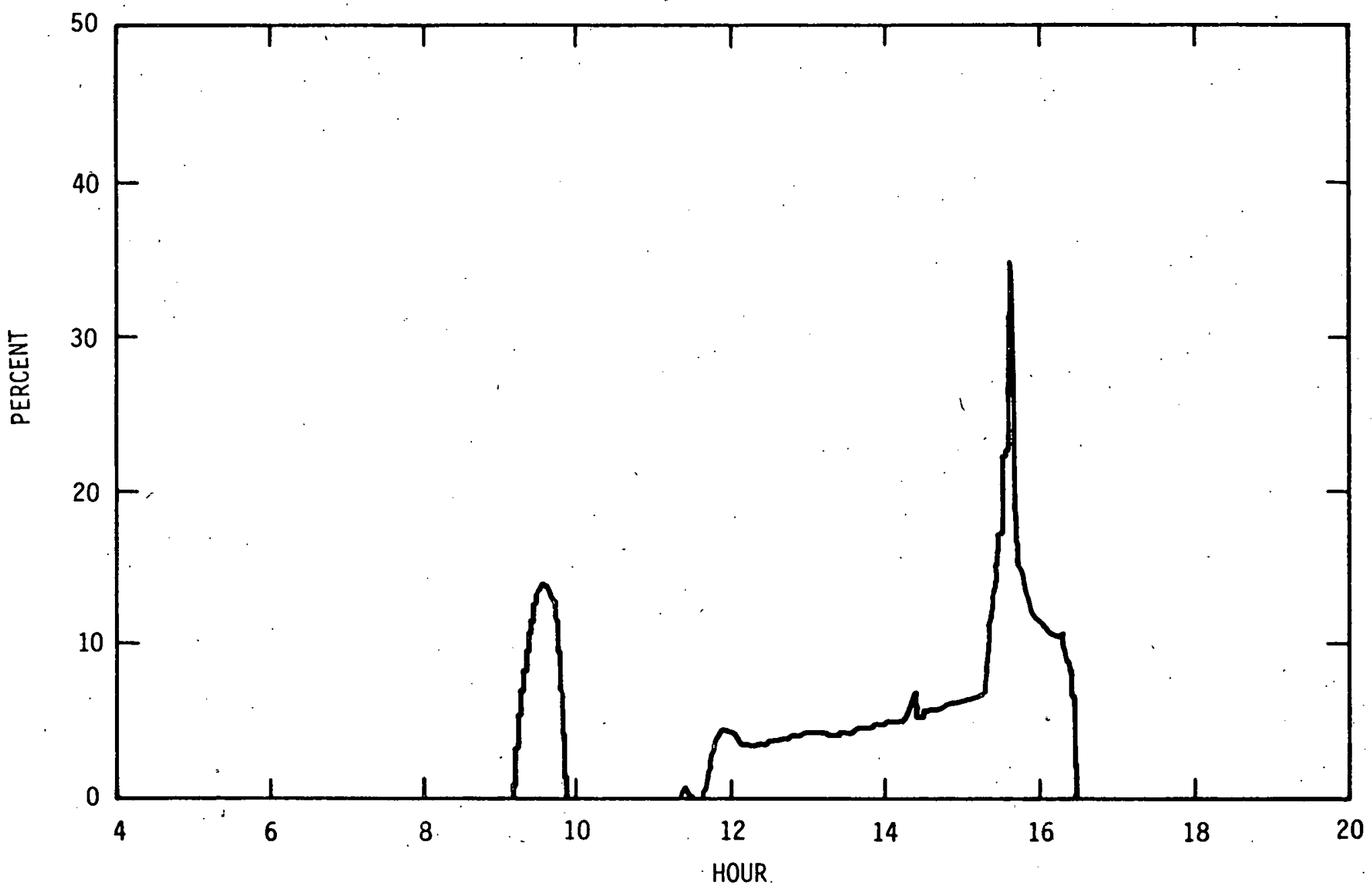

Figure 4. Efficiency, $351=1979$ 


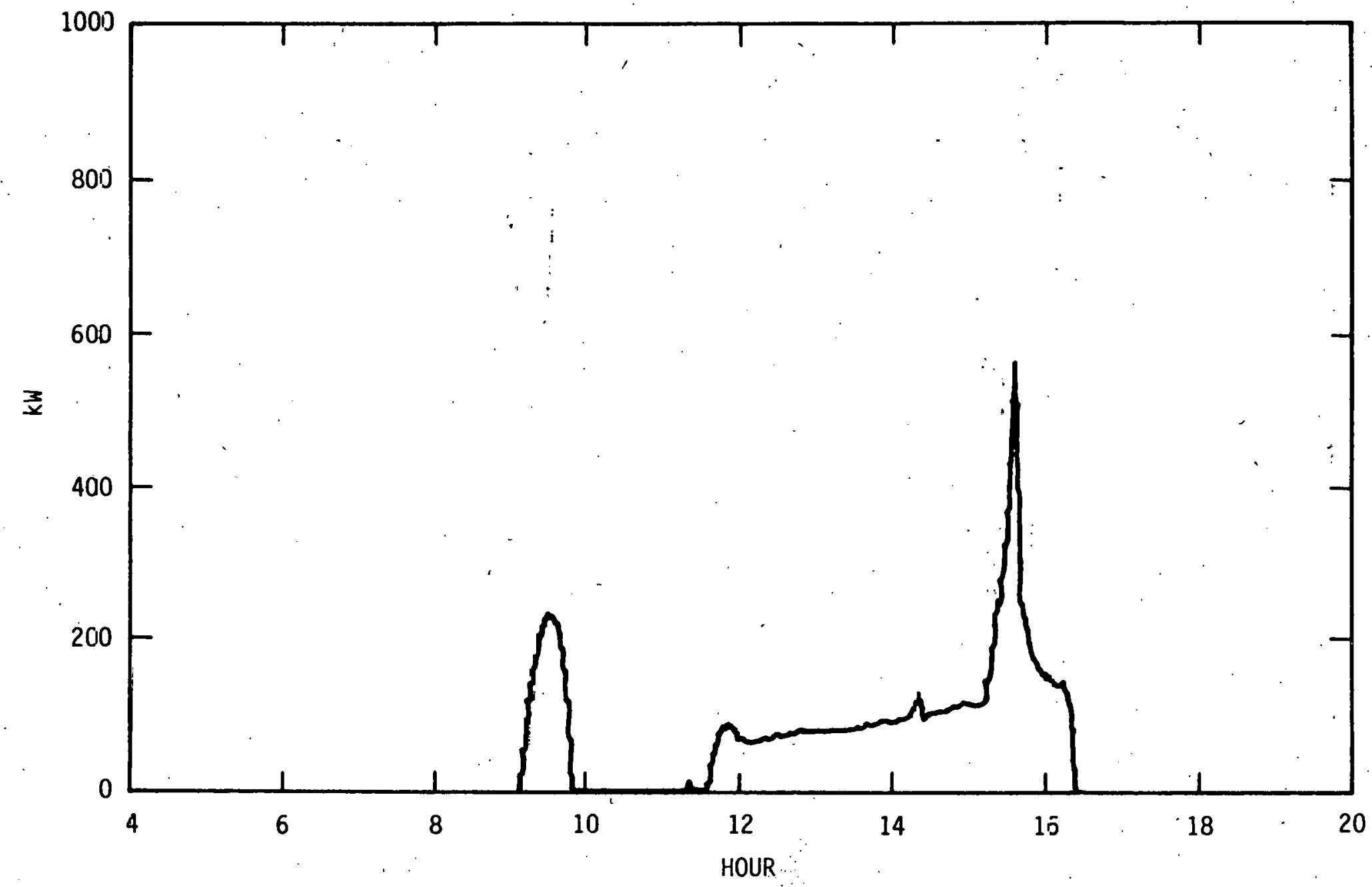

Figure 5. Collected Power, 351-1979 


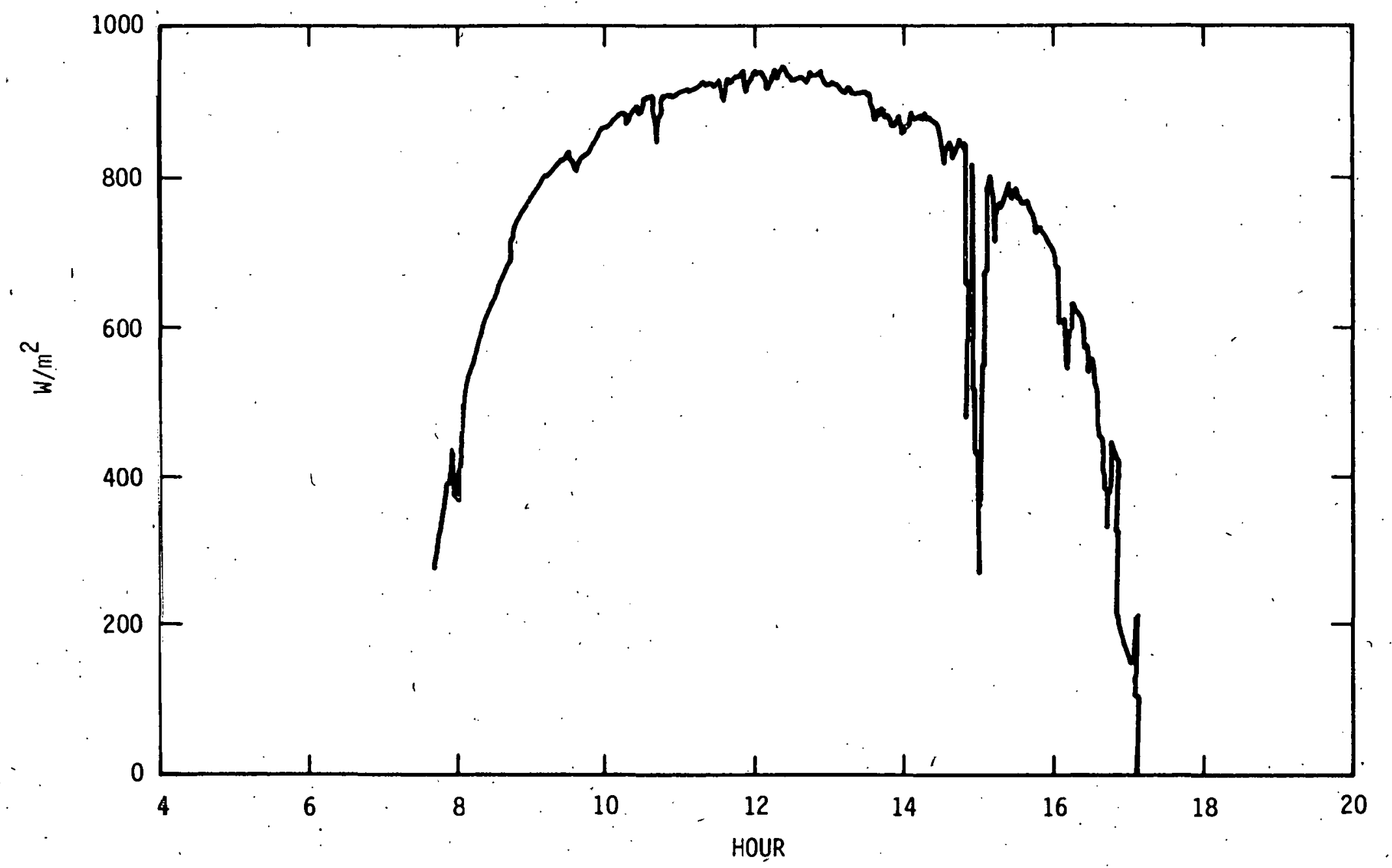

Figure 6. Insolation, 352-1979 


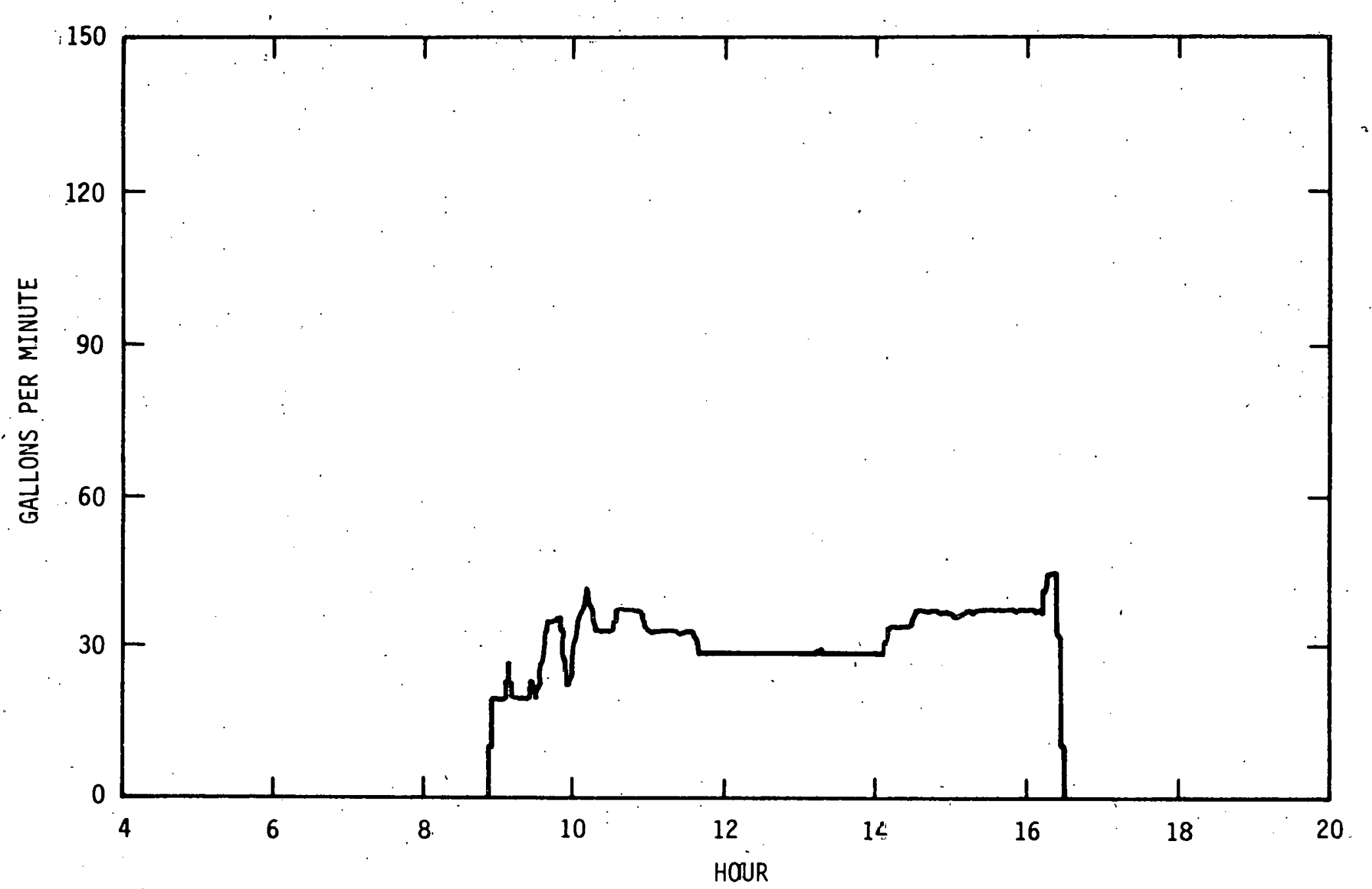

Iigure 7. Flow Rate, 352-1979 


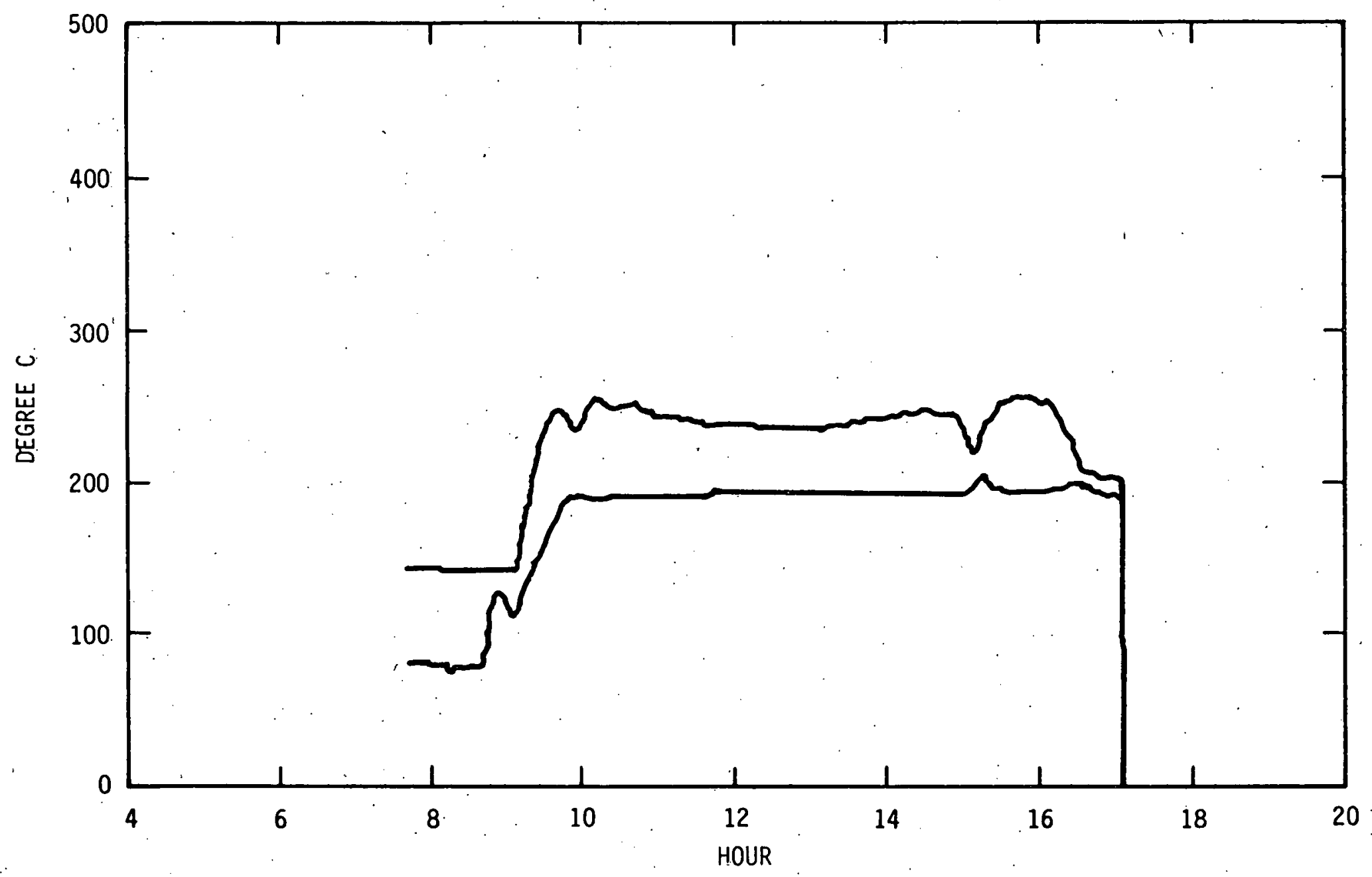

Figure 8. Inlet and Outlet Temperatures, 352-1979. 
a

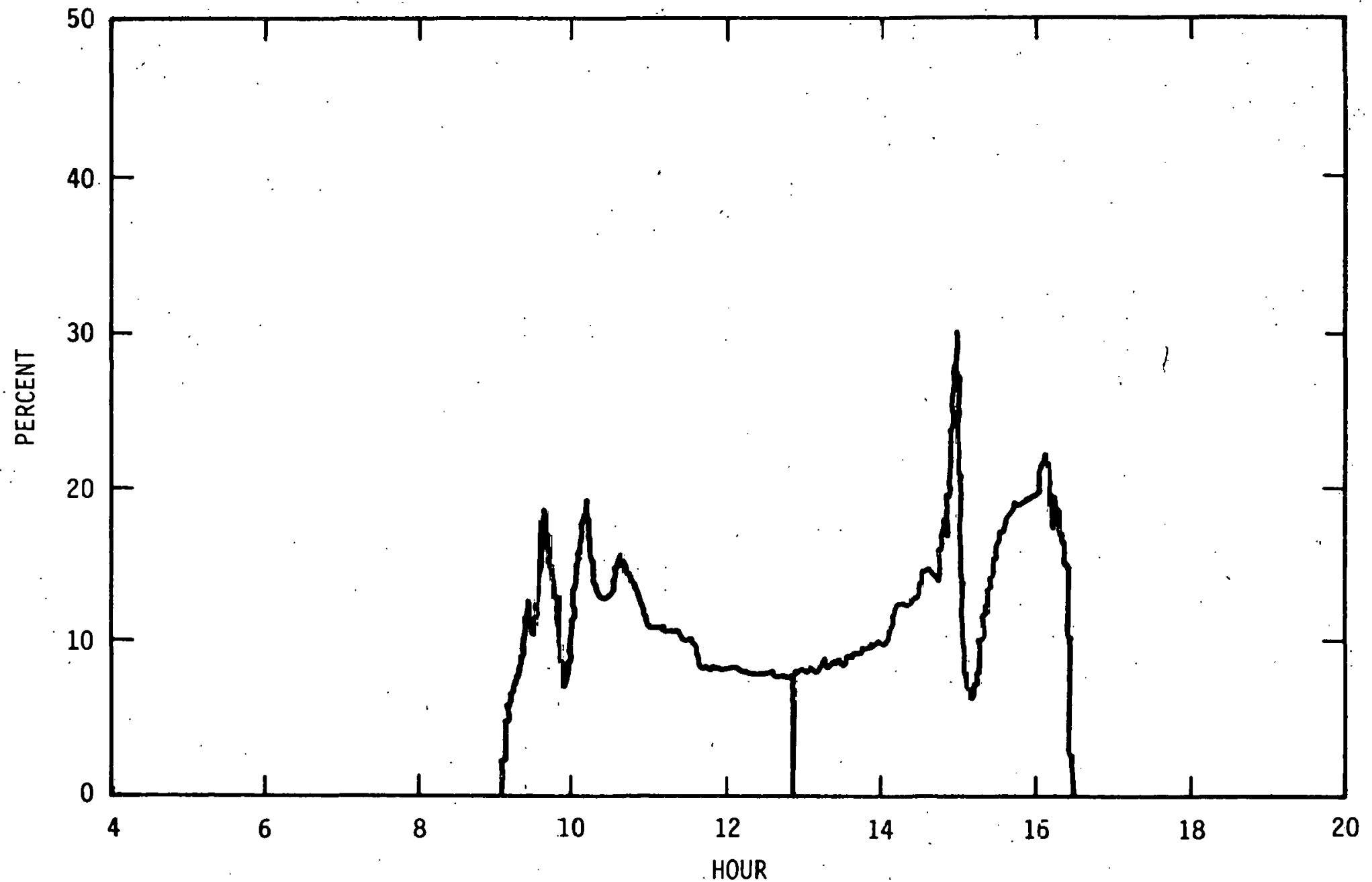

Figure 9. Efficiency, 352-1979 


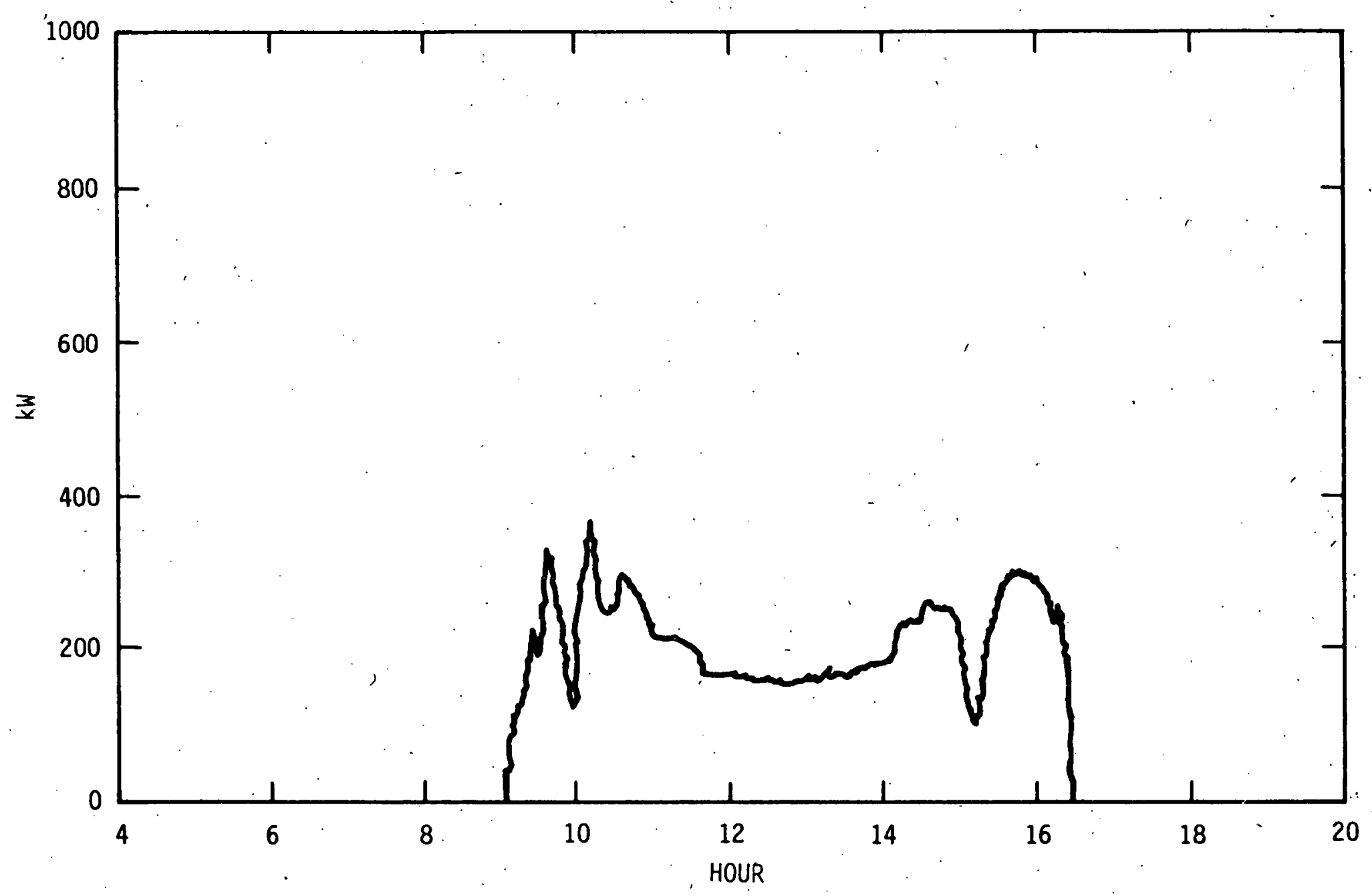

Figure 10. Collected Power, 352-1979 


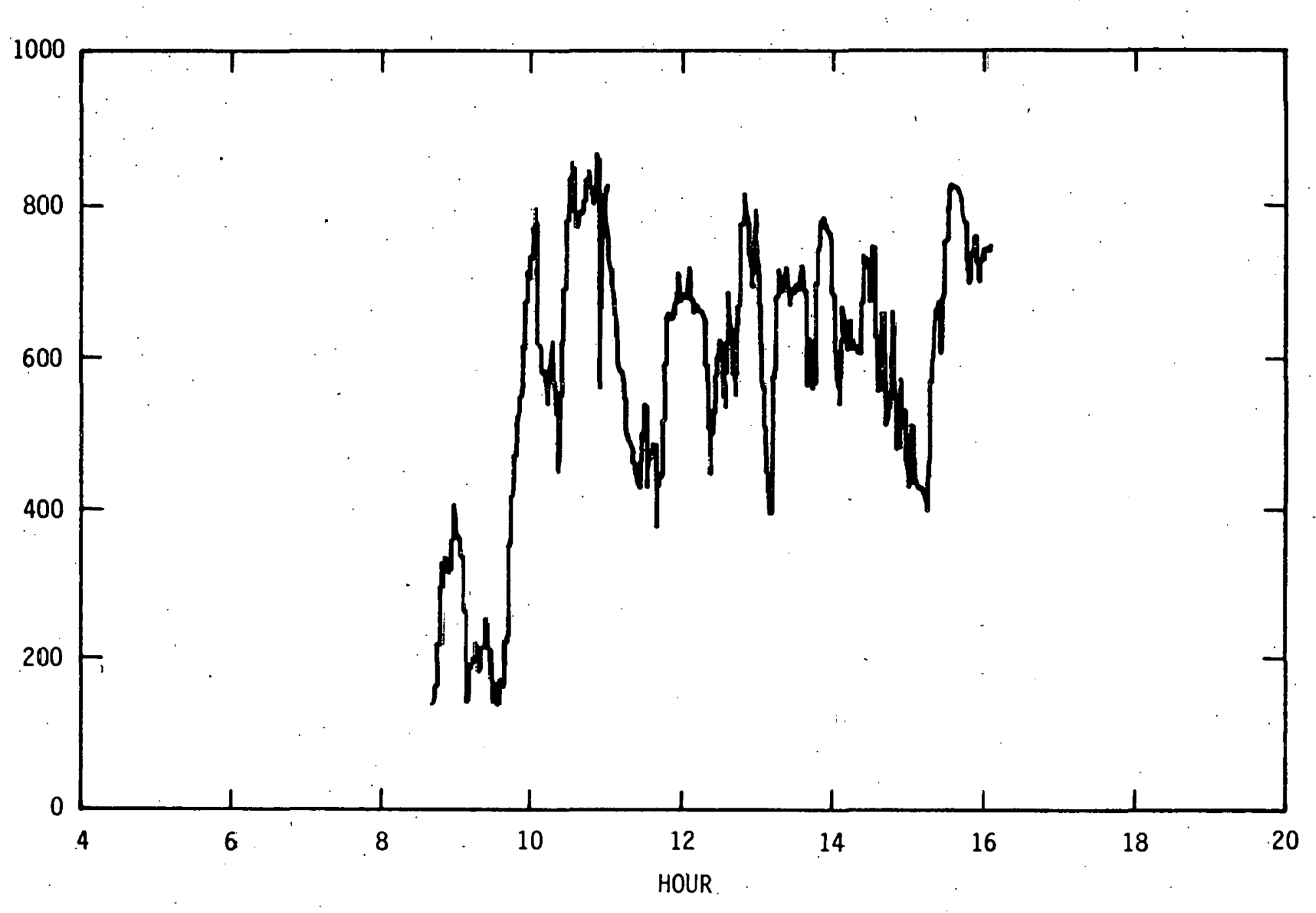




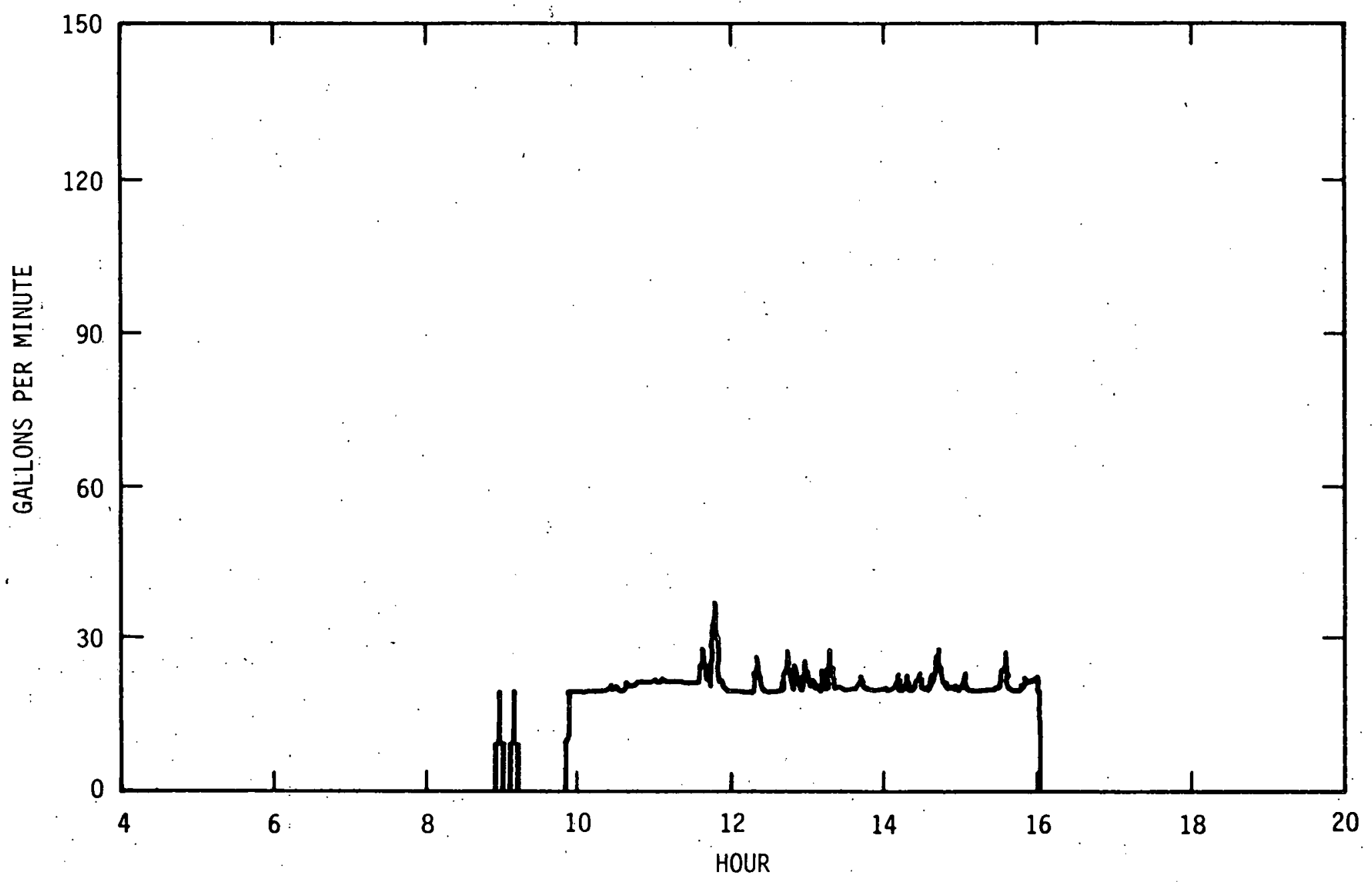

Figure 12. Flow Rate, 358-1979 


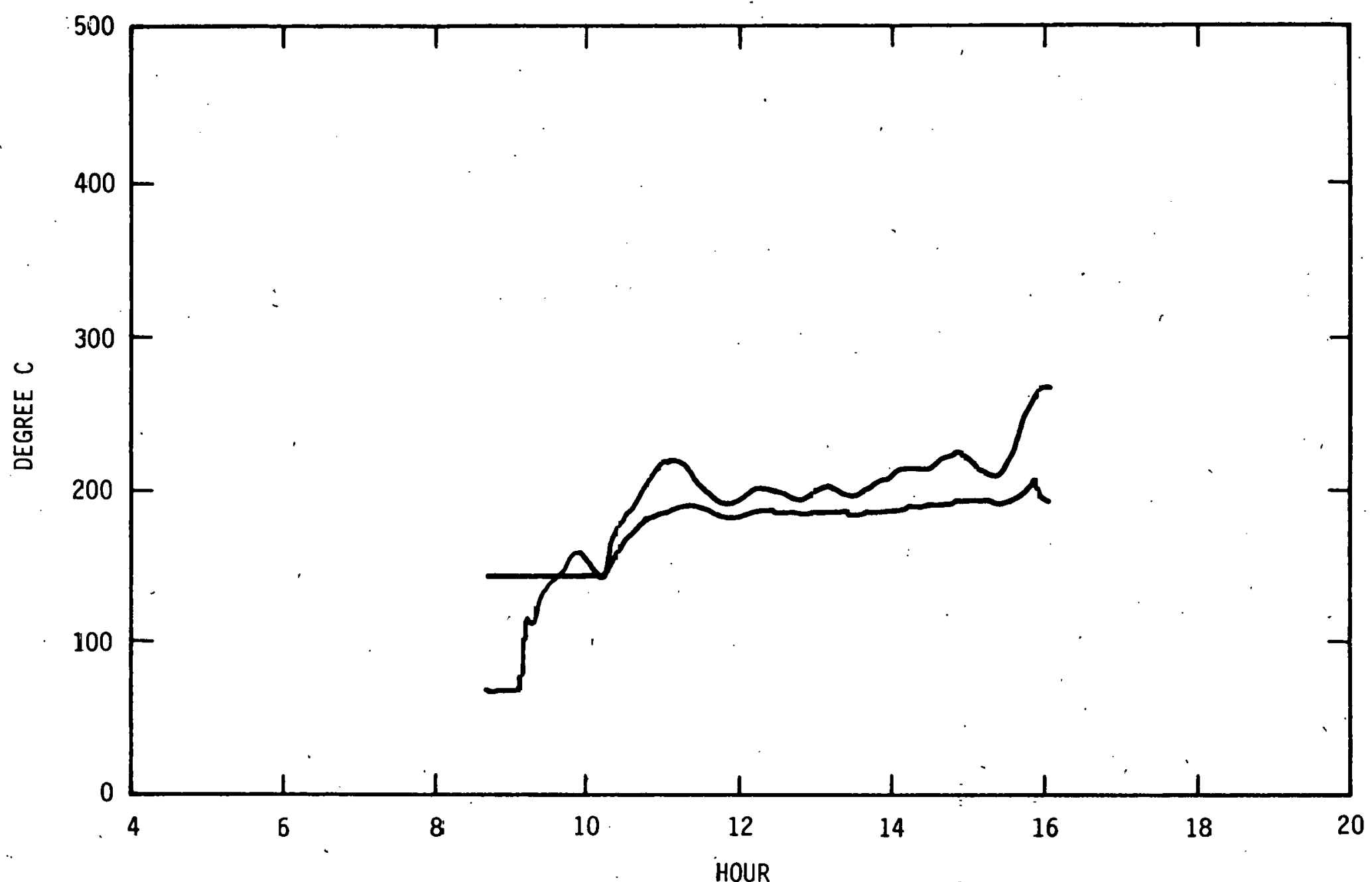

Figure 13. Inlet and outlet Temparatures, 358-1979. 


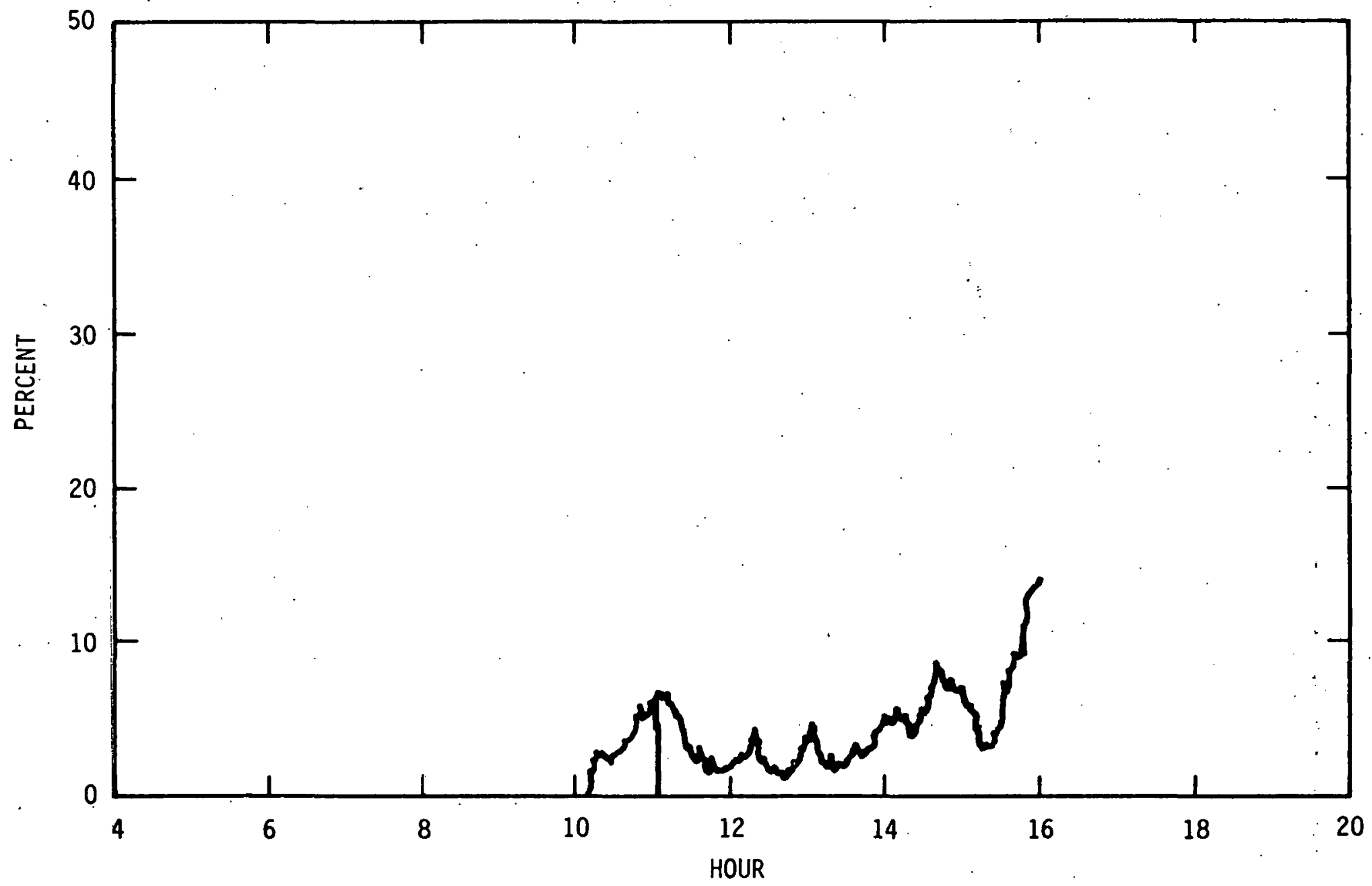

Figure 14. Efficiency, 358-1979. 


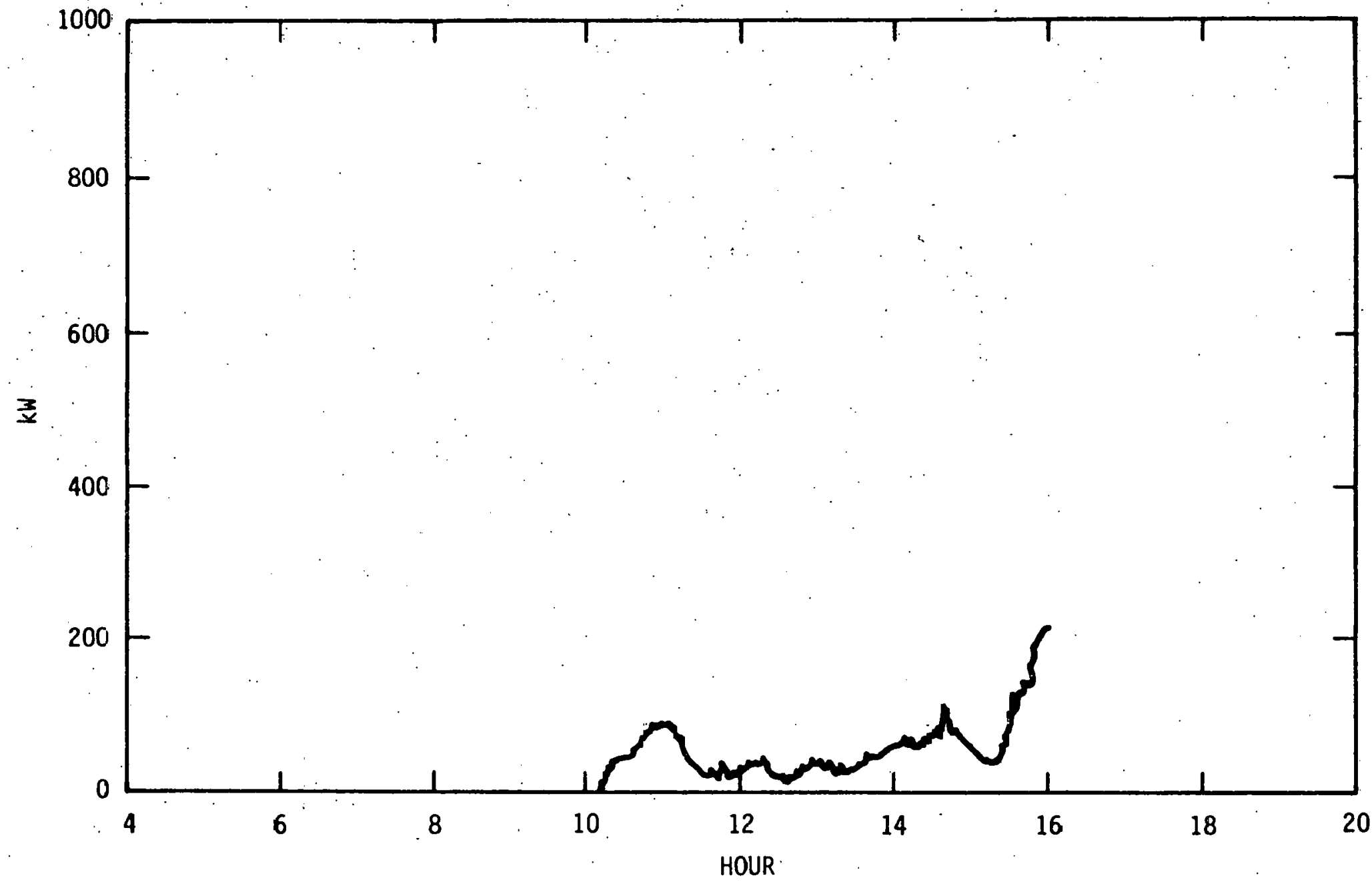

Figure 15. Collected Power, 35E-1979 
SOLAR COLLECTOR SYSTEM VERNAL EQUINOX PERFORMANCE, 20 AND 23 MARCH AND 3 APRIL 1980

Solar collector performance tests were conducted on 20 and 23 March and 3 April 1980 (days 80, 83, and 94) to determine solar energy collection efficiency. during the period near spring equinox. Average collection efficiencies for those days were $22.98,26.98$, and 30.18 , respectively.

\section{Methods}

Collector reflective surfaces and receiver insulating glass tubes were washed, and tracking was adjusted to align reflection onto the receiver tubes prior to commencing the performance tests. Washing and rinsing by a commercial firm was repeated before the 3 April test.

After a lower flow rate startup period to preheat the system, Caloria was circulated from the main storage tank at a flow rate which maintained the desired collector system outlet temperature. Inlet temperature was about $200^{\circ} \mathrm{C}\left(392^{\circ} \mathrm{F}\right)$; outlet temperature was $260^{\circ}$ to $288^{\circ} \mathrm{C}\left(500^{\circ}\right.$ to $\left.550^{\circ} \mathrm{F}\right)$ during the various tasks.

All test periods had good insolation and moderate ambient temperatures and wind velocities. See Table $l$ for the times recorded for the events of each test day.

Collector system flow rate was measured with a vortex-type device, temperatures with resistance temperature detector (RTD) and thermocouple sensors, and insolation with a pyrheliometer. 


\section{Table 1}

Times Recorded on Days of Spring

Equinox Collector Tests

\begin{tabular}{|c|c|c|c|}
\hline Event & March 20 & March 23 & April 3 \\
\hline Sunrise & $6: 28$ a.m. & $6: 20, \mathrm{a} \cdot \mathrm{m}$. & $6: 14 \mathrm{a} \cdot \mathrm{m}$. \\
\hline $\begin{array}{l}\text { Collectors } \\
\text { Focused }\end{array}$ & $7: 38$ a.m. & $7: 34 \mathrm{a} \cdot \mathrm{m}$ & $7: 20$ a.m. \\
\hline $\begin{array}{l}\text { Switch to } \\
\text { Main Tank }\end{array}$ & $8: 58$ a.m. & $8: 29 \mathrm{a} \cdot \mathrm{m}$ & $8: 08$ a.m. \\
\hline $\begin{array}{l}\text { Collectors } \\
\text { Defocused }\end{array}$ & $5: 15 \mathrm{p} \cdot \mathrm{m}$ & $5: 32 \quad p=m$ & $5: 42 \mathrm{p} \cdot \mathrm{m}$, \\
\hline Sunset & $6: 35 \mathrm{p} \cdot \mathrm{m}$. & 6:35 p.m. & $6: 47 \mathrm{p}: \mathrm{m}$. \\
\hline
\end{tabular}

Collector system efficiency was computed as thermal energy gained by Caloria between system inlet and outlet manifold locations divided by direct normal solar radiation times the collector aperture area.

\section{Conclusions}

The collection efficiency at vernal equinox was shown to be significantly higher than at winter solstice. This was as expected, since the incidence angles between the sun and the collector apertures are much. less at vernal equinox than at winter solstice. At equinox, the sun is exactly normal to the collectors at sunrise and sunset and less than normal at noon, which explains the shape of the efficiency curves.

Performance test results for days 80,83, and 94 follow. 
Day 80 Results

Solar collector system efficiency on day 80 ranged from 238 to $29 \%$ for much of the central part of the test period. "The average collection efficiency for the entire day was 22.98 .

Day 80 was clear with modest winds which briefly ranged up to $10 \mathrm{~km} / \mathrm{h}(6.2 \mathrm{mi} / \mathrm{h})$. Ambient temperature was about $20^{\circ} \mathrm{C}\left(68^{\circ} \mathrm{F}\right)$ during much of the test period.

Collector system outlet temperature was maintained at about $280^{\circ} \mathrm{C}$ $\left(535^{\circ} \mathrm{F}\right)$ during most of the test. Inlet temperature was about $225^{\circ} \mathrm{C}$ $\left(437^{\circ} \mathrm{F}\right)$. Collector system Caloria flow rate was quite variable but was about $5 \mathrm{l} / \mathrm{s} .(79 \mathrm{gal} / \mathrm{min})$ for much of the test period. 


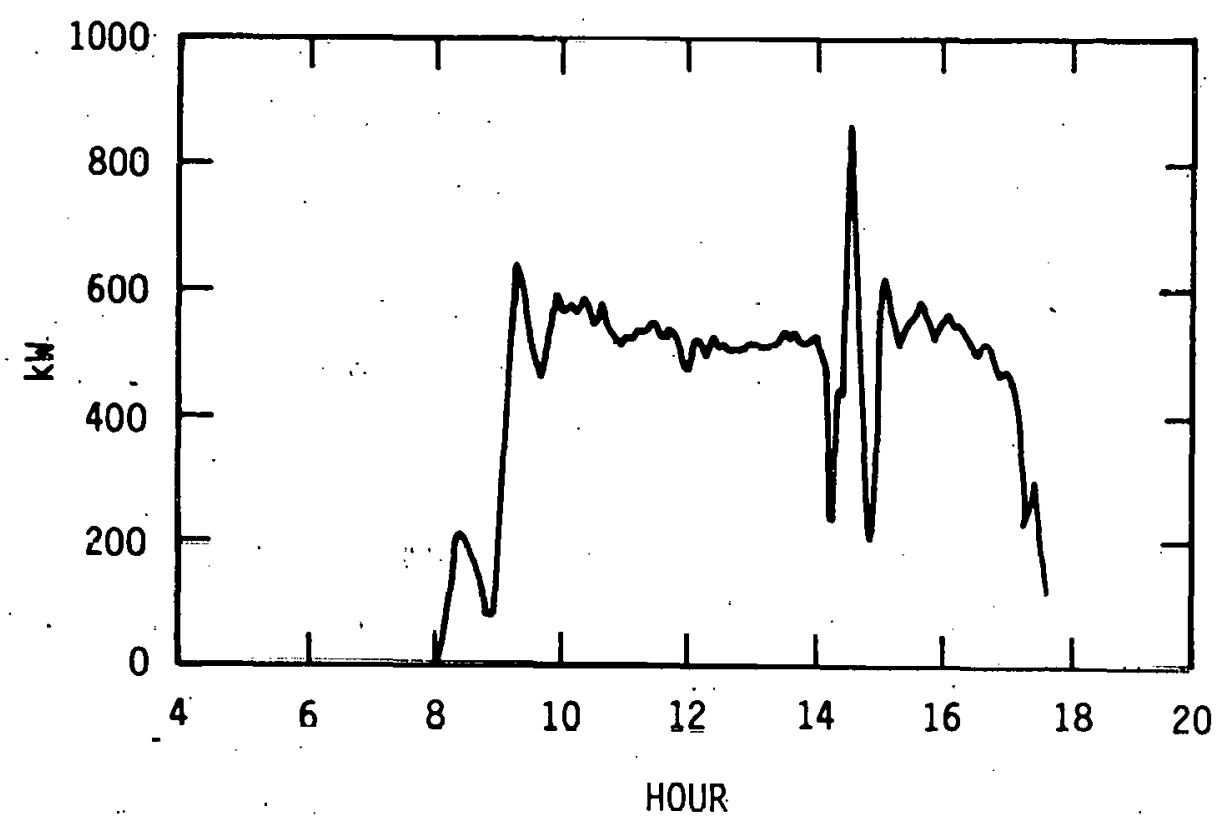

Figure 1. Collected Power, 80-1980

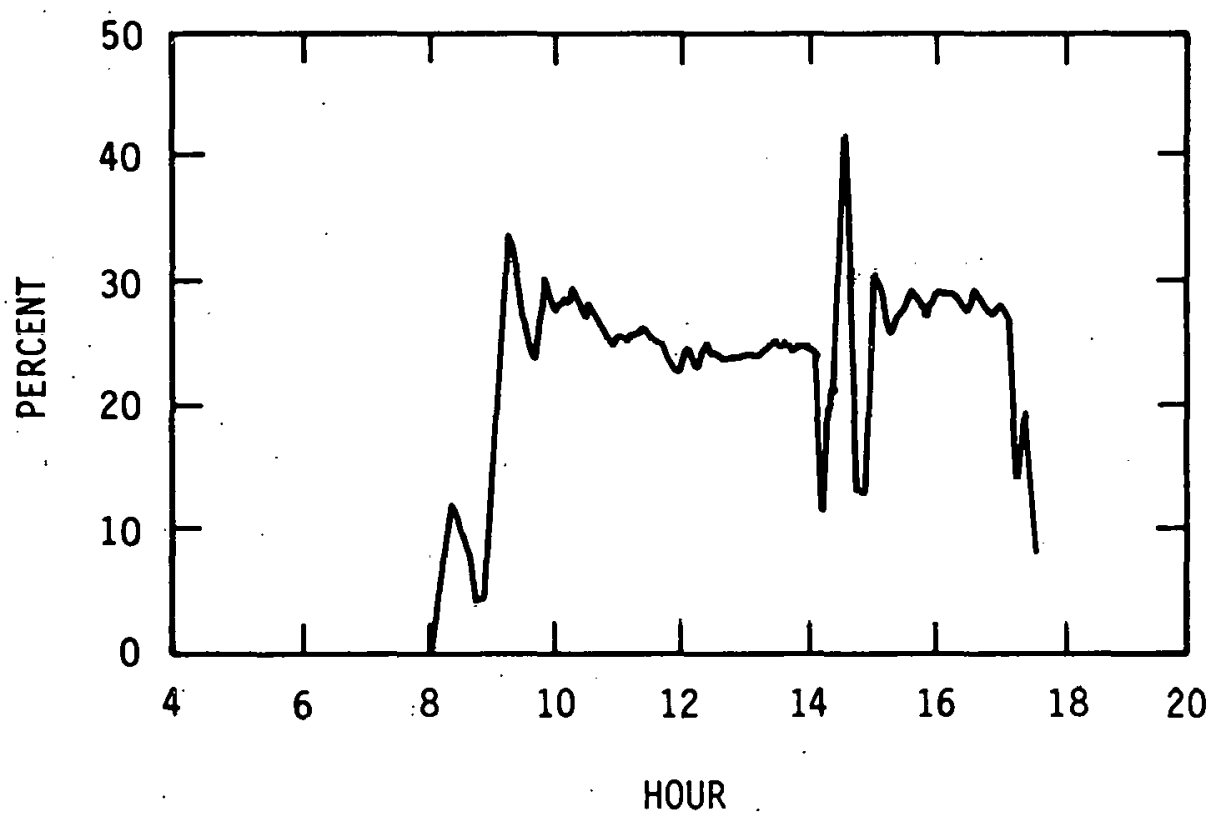

Figure 2. Efficiency, 80-1980 


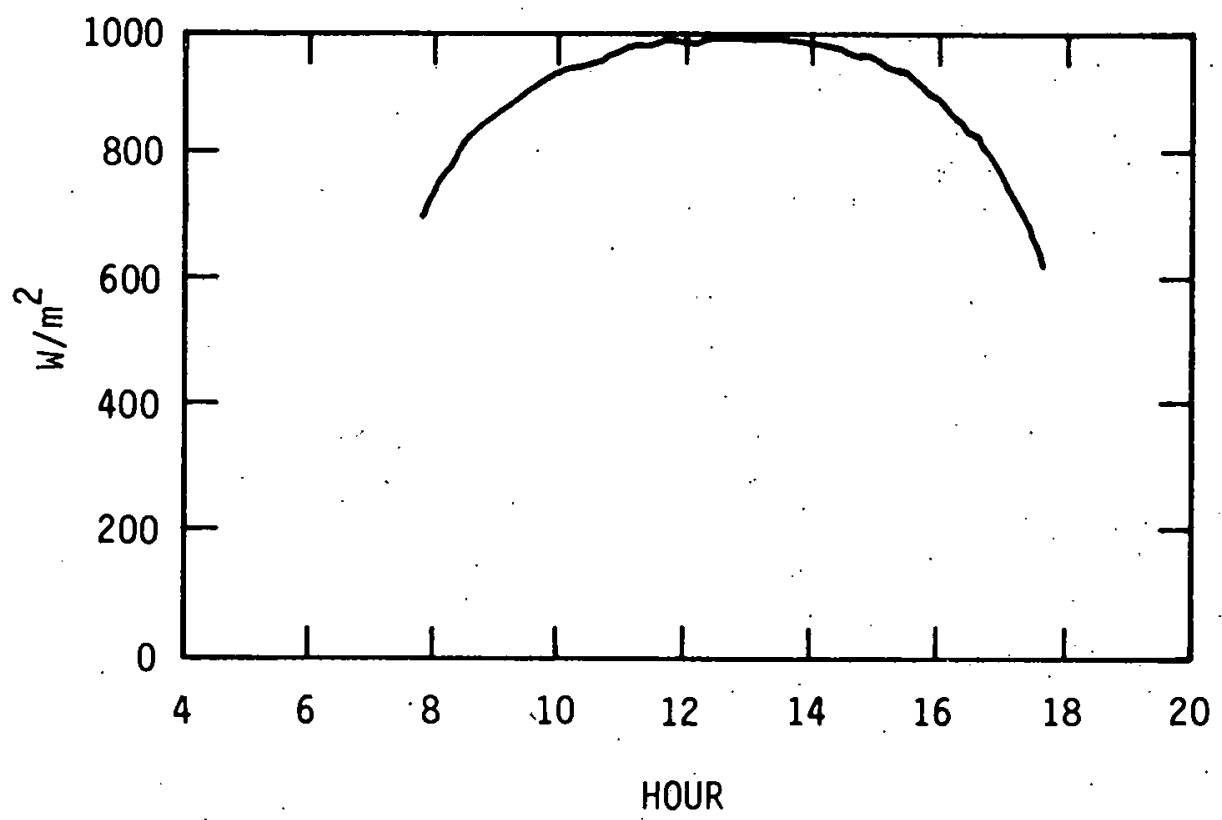

Figure 3. Insolation, 80-1980

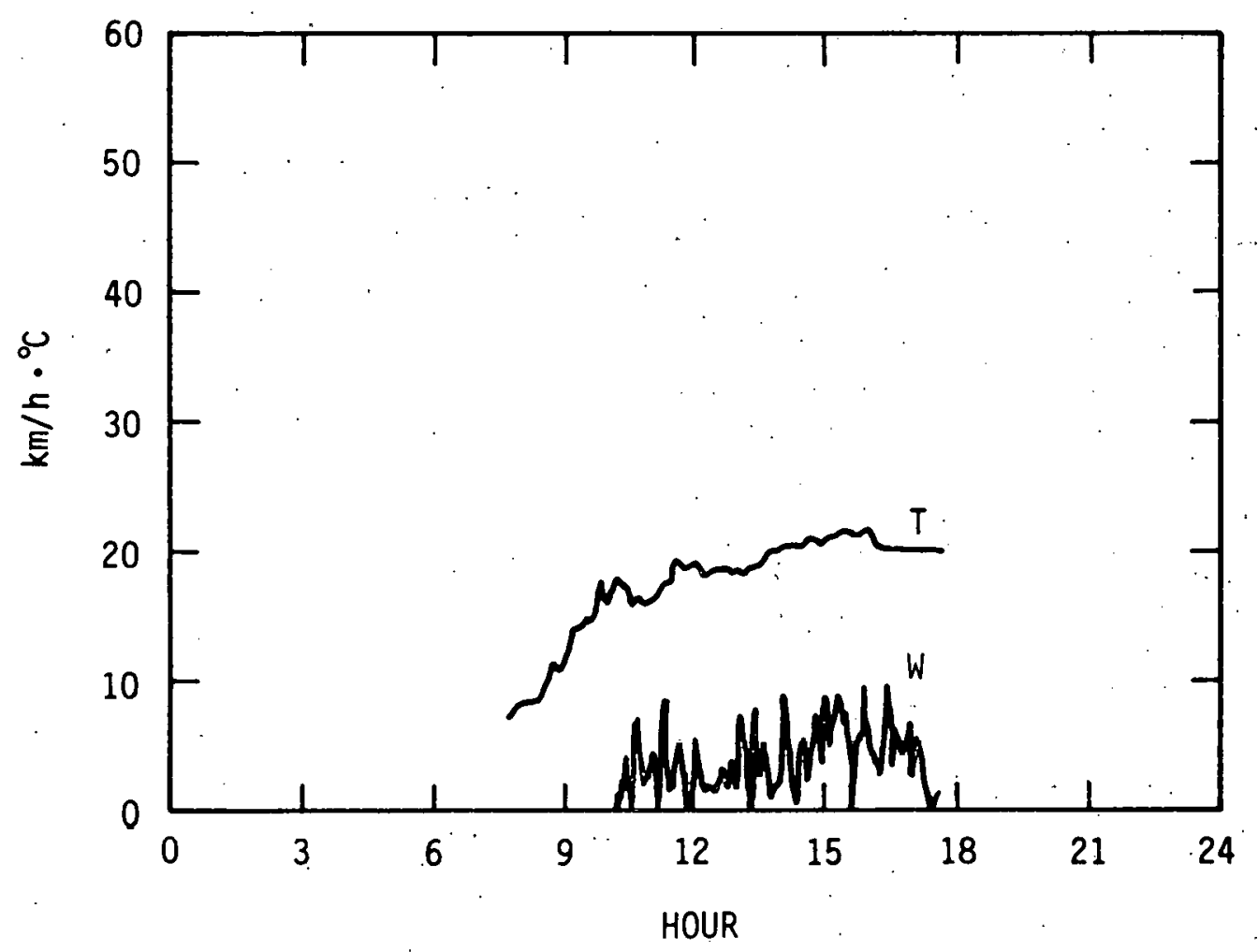

Figure 4. Wind speed and Ambient Temperature, 80-1980 


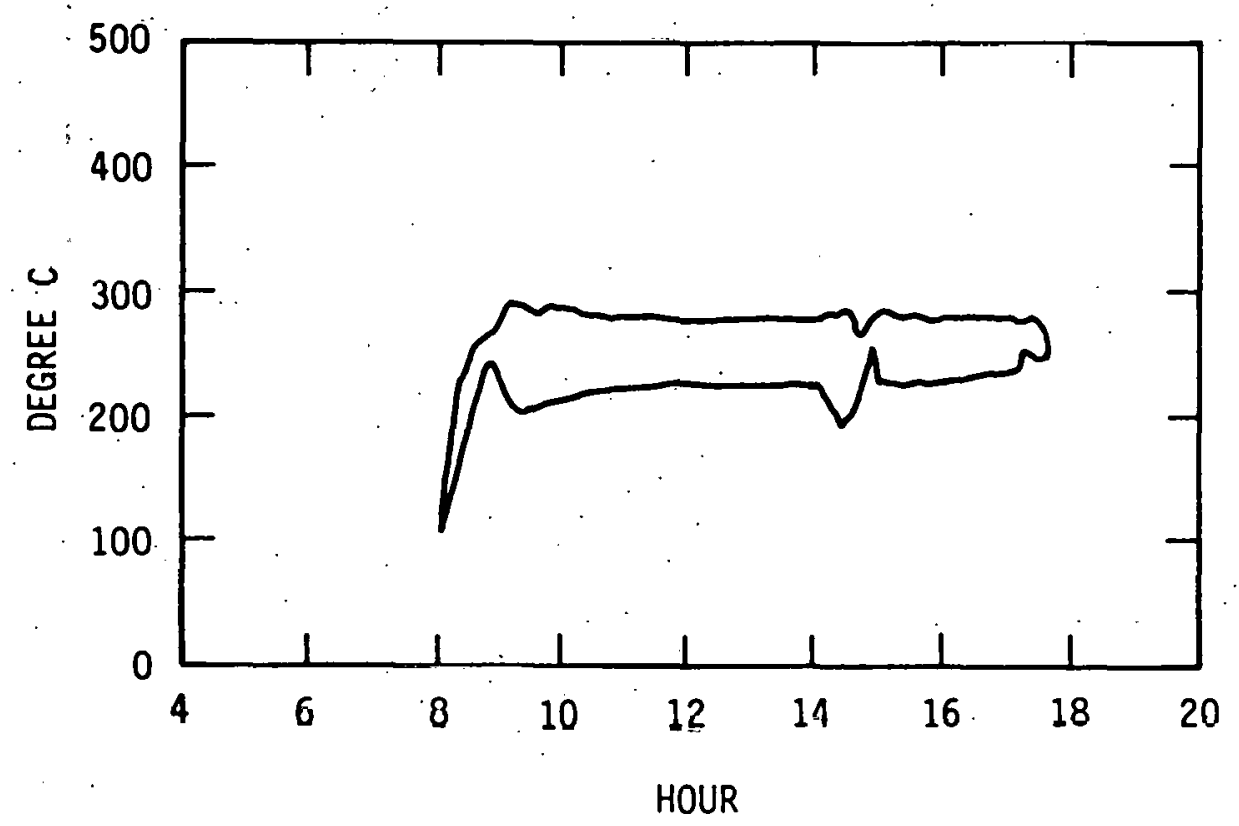

Figure 5. Inlet and outlet Temperatures, 80-1980

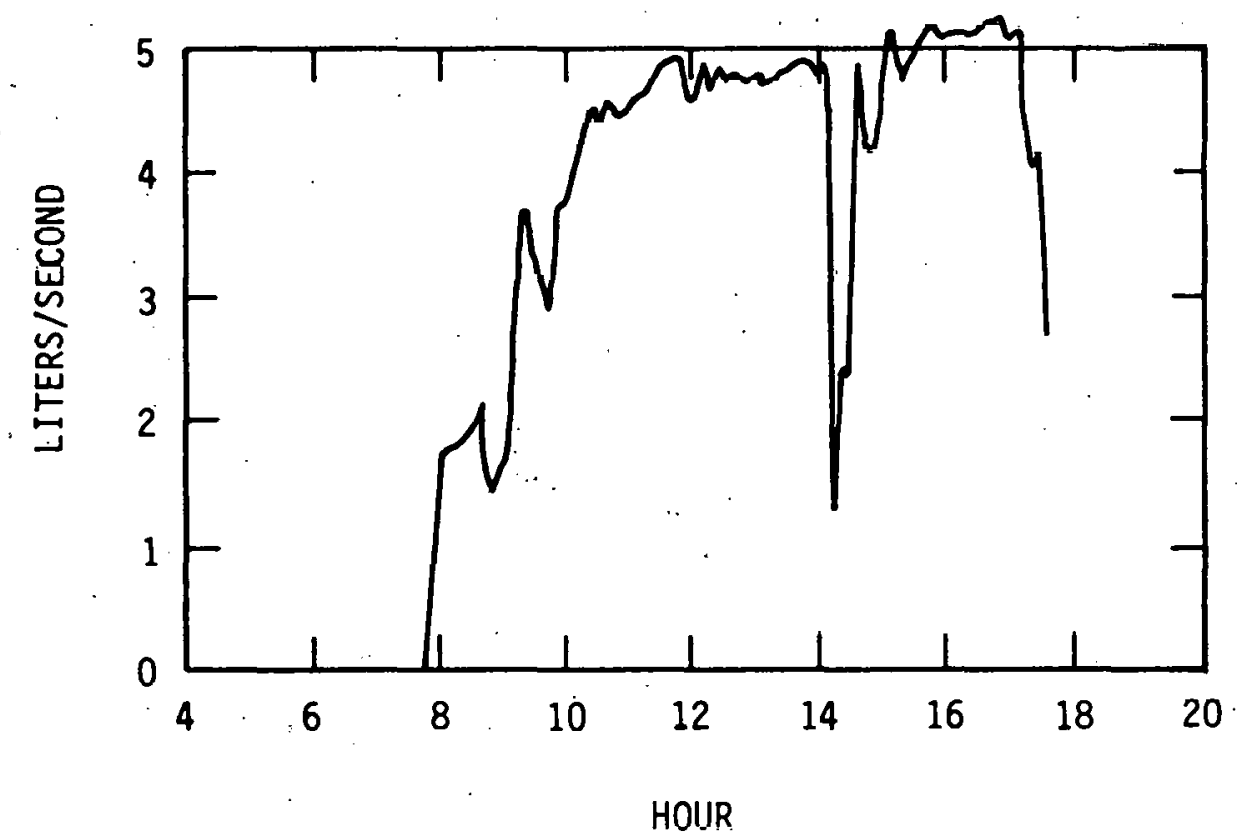

Figure 6. Flow Rate, 80-1980 


\section{Day 83 Results}

On day 83, collector system efficiency ranged from $25 \%$ to $30 \%$ except for the periods immediately after startup. and before shutdown. The average daily solar energy collection efficiency was 26.98 .

Day. 83 was clear with essentially no wind. However, wind velocity was not recorded. Ambient temperature was. $12^{\circ}$ to $20^{\circ} \mathrm{C} .\left(54^{\circ}\right.$ to $68^{\circ} \mathrm{F}$ ).

The collector system outlet temperature was maintained at about $260^{\circ} \mathrm{C}\left(500^{\circ} \mathrm{F}\right)$. System flow rate was about $5 \mathrm{l} / \mathrm{s}(79 \mathrm{gal} / \mathrm{min})$ from $10 \mathrm{a} \cdot \mathrm{m}$. until $2 \mathrm{p} . \mathrm{m}$. and then increased to nearly $6 \mathrm{l} / \mathrm{s}(95 \mathrm{gal} / \mathrm{min})$. for the remainder of the day to maintain the desired collector system outlet temperature. 


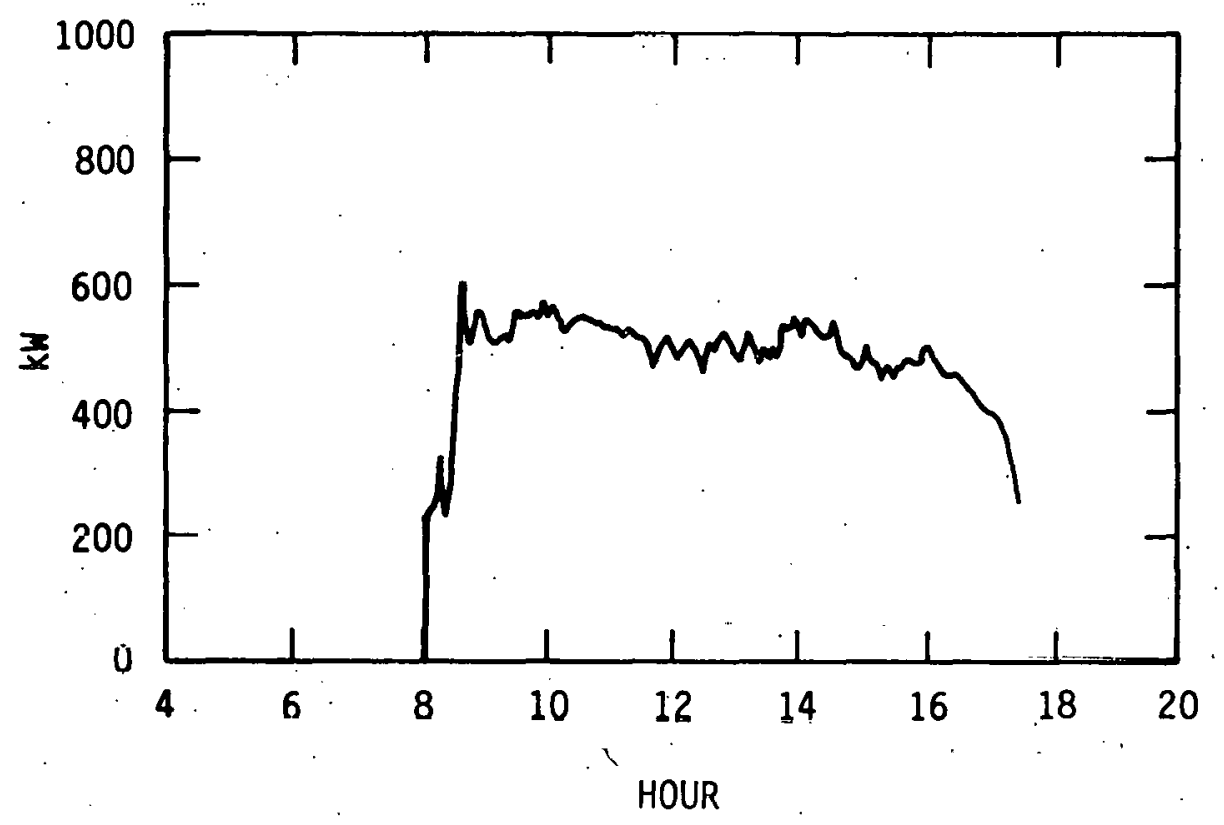

Figure 7. Collected Power, 83-1980

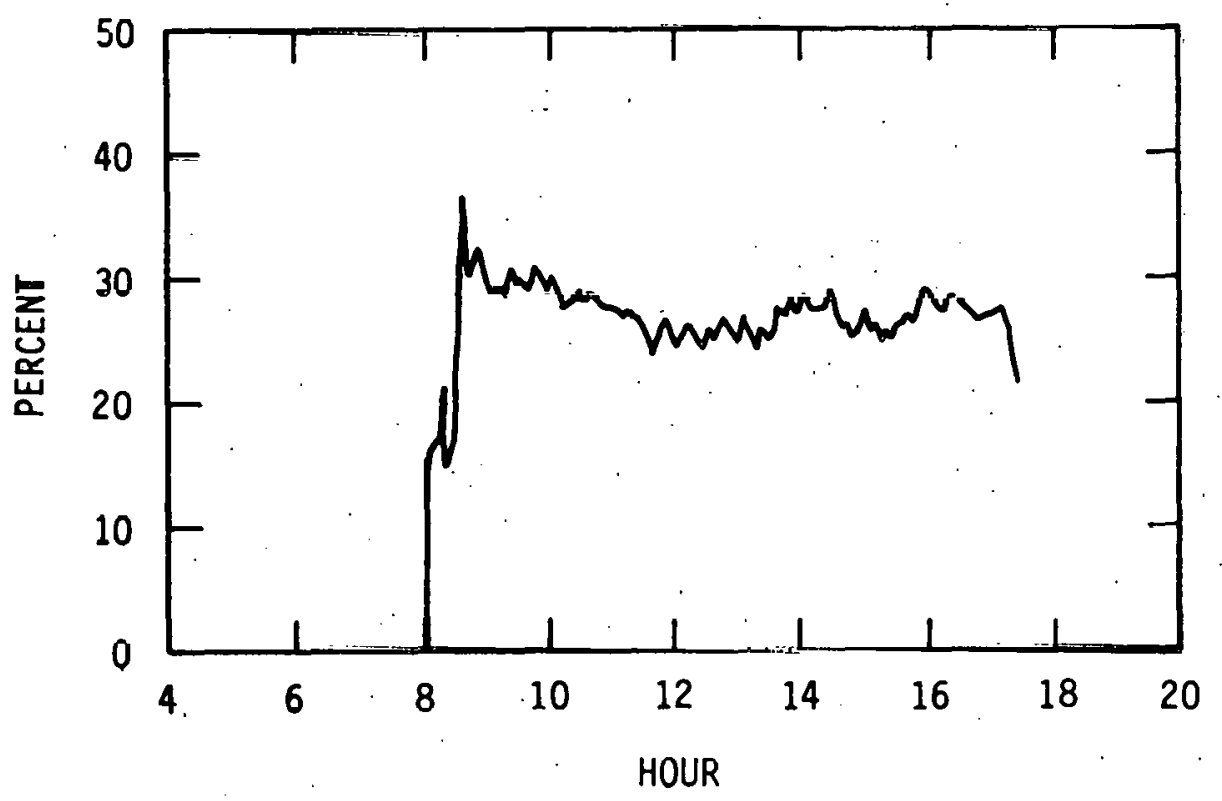

Figure 8. Efficiency, 83-1980. 


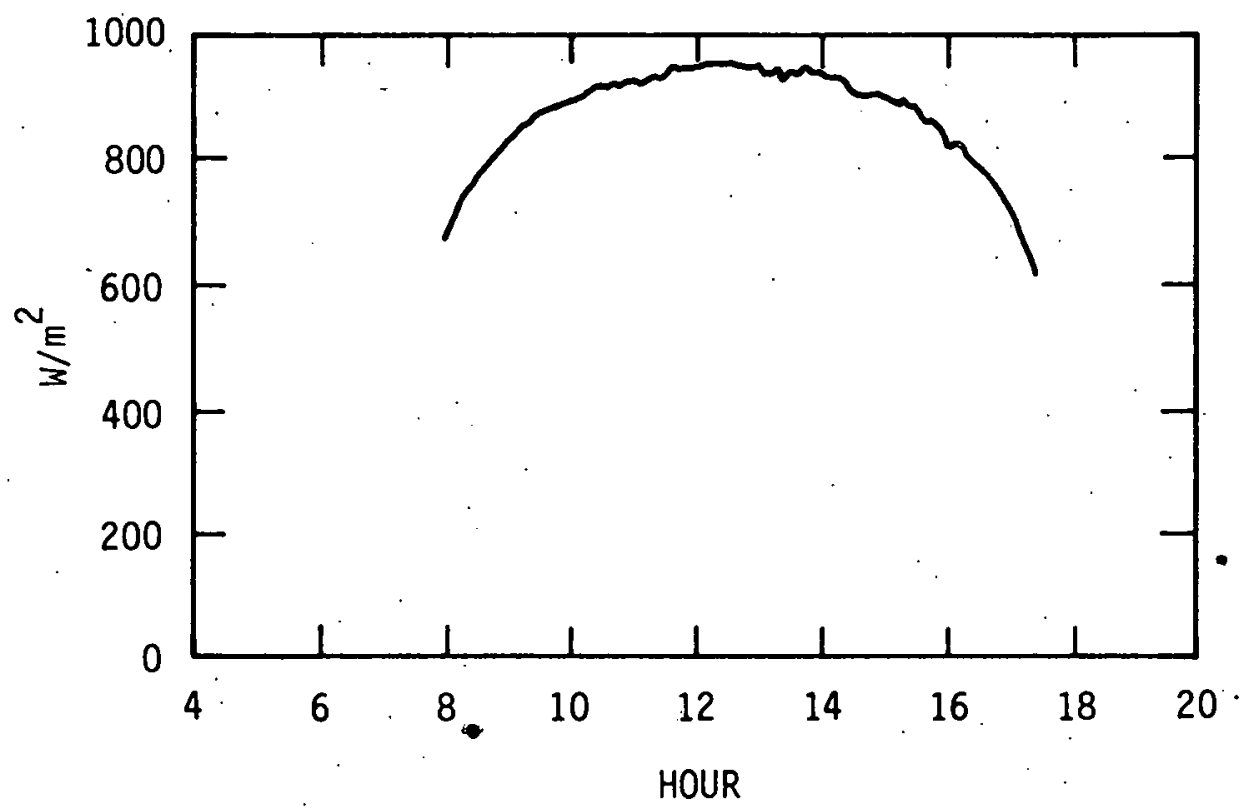

Figure 9. Insolation, 83-1980.

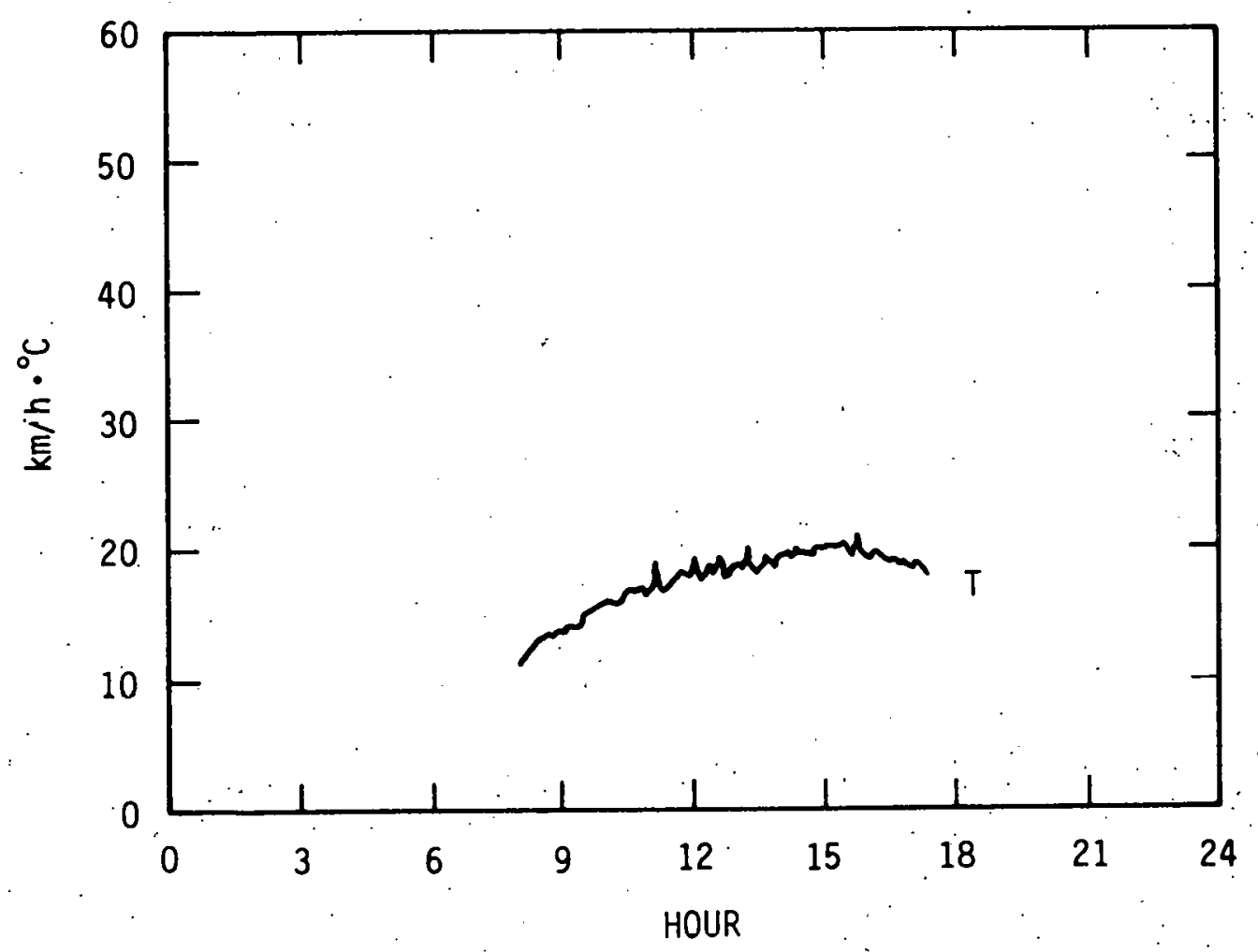

Figure 10. Wind Speed and Ambient Temperature, 83-1980. 


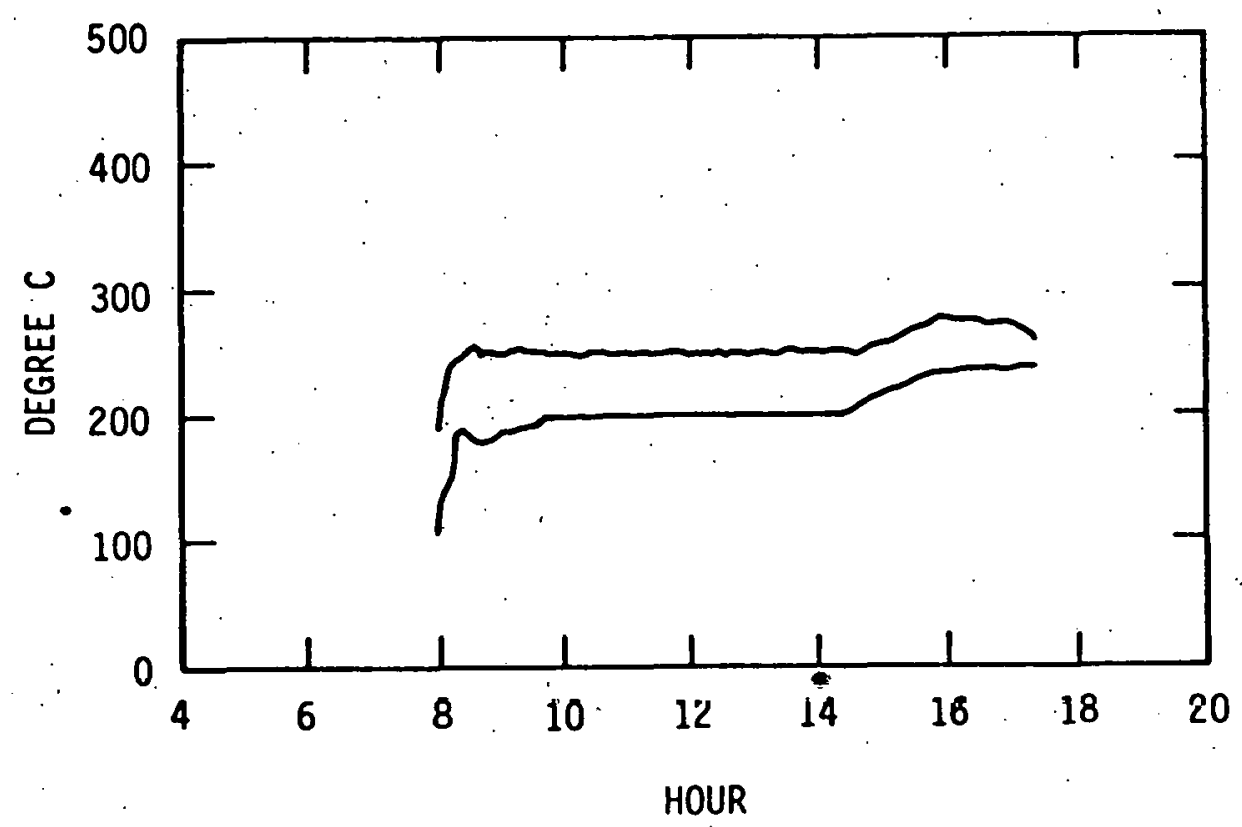

Figure 11. Inlet and Outlet Temperatures, 83-1980

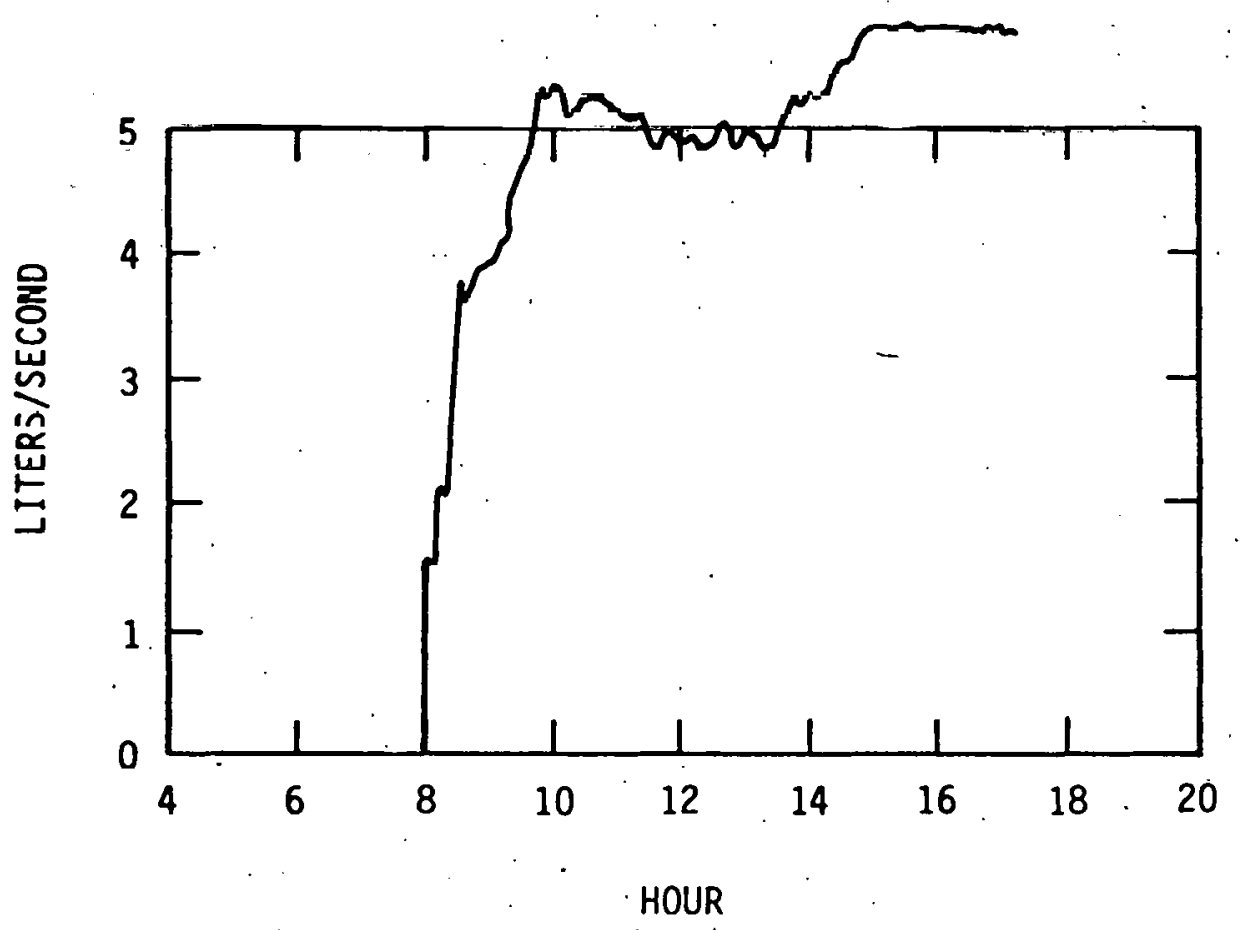

Figure 12. Flow Rate, 83-1980 
Day 94 Results

Solar collector surfaces were washed and rinsed thoroughly by a commercial firm prior to the day 94 collector system performance test.

Collector system solar energy gathering efficiency ranged from $30 \%$ to $37 \%$ during the period from 9 . a.m. to $3 \mathrm{p} . \mathrm{m}$. The average collection efficiency during the day was $30.1 \%$.

Day 94 was clear until after $4 \mathrm{p} . \mathrm{m}$. Ambient temperature was only about $15^{\circ} \mathrm{C}\left(61 .^{\circ} \mathrm{F}\right)$ at $10 \mathrm{a} \cdot \mathrm{m}$. but increased to between $20^{\circ}$ and $23^{\circ} \mathrm{C}$ $\left(68^{\circ}\right.$ and $73^{\circ} \mathrm{F}$ ) later in the test period. Wind velocities were highly variable, averaging about $5 \mathrm{~km} / \mathrm{h}(3 \mathrm{mi} / \mathrm{h})$. with brief wind bursts of over $10 \mathrm{~km} / \mathrm{h}(6 \mathrm{mi} / \mathrm{h})$ on four occasions.

The collector system outlet temperature was maintained at $285^{\circ} \mathrm{C}$ $\left(545^{\circ} \mathrm{F}\right)$ throughout the test. Inlet temperature was about $190^{\circ}$ to $200^{\circ} \mathrm{C}\left(374^{\circ}\right.$ to $\left.392^{\circ} \mathrm{F}\right)$. System flow rate was about $3.4 \mathrm{l} / \mathrm{s}$ (45 $\mathrm{gal} / \mathrm{min})$, but varied somewhat to maintain the desired outlet temperature. 


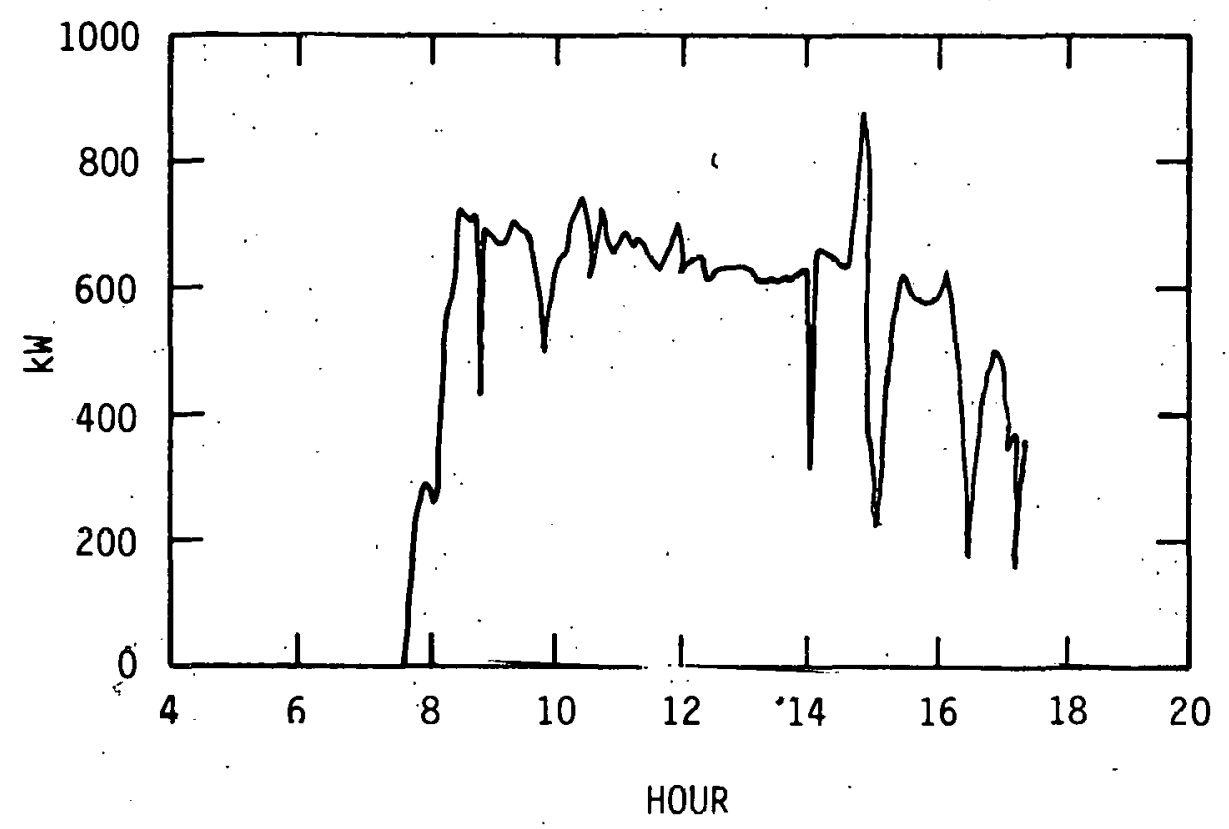

Figure 13. Collected Power, 94-1980

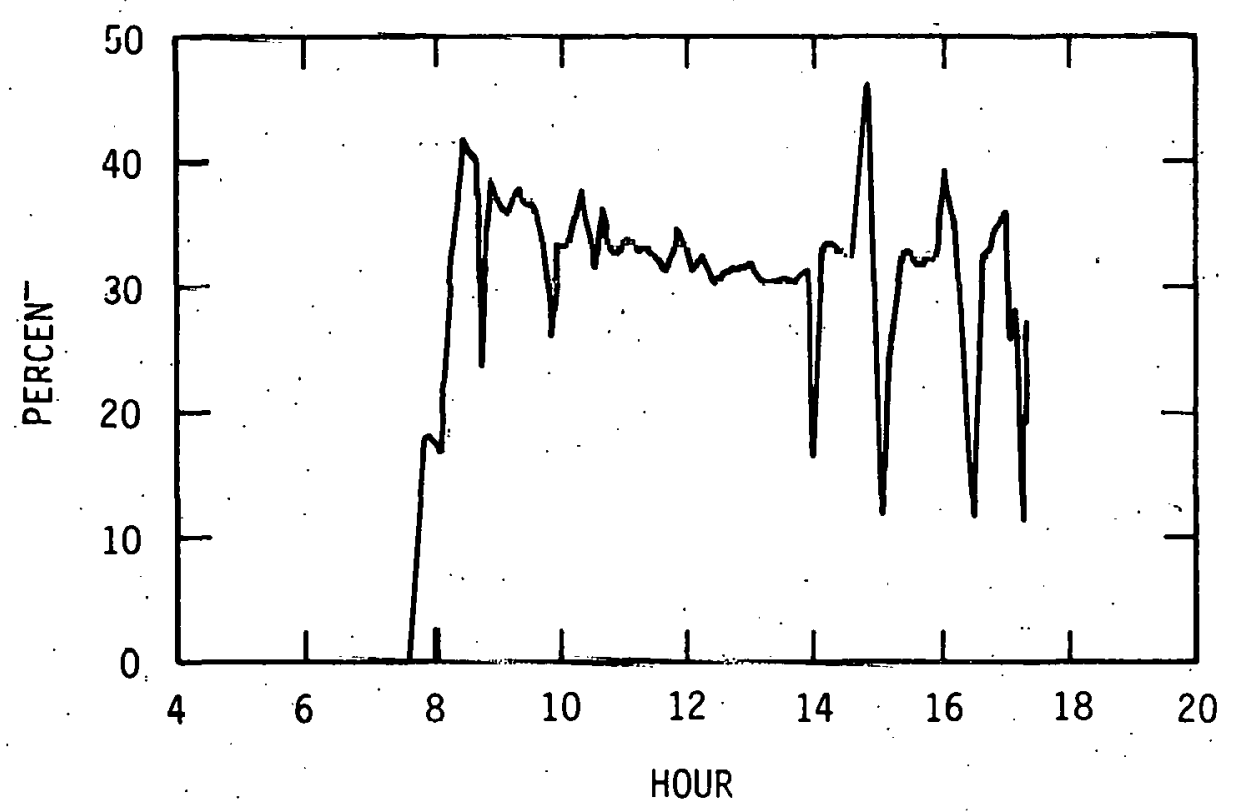

Figure 14. Efficiency, 94-1980 


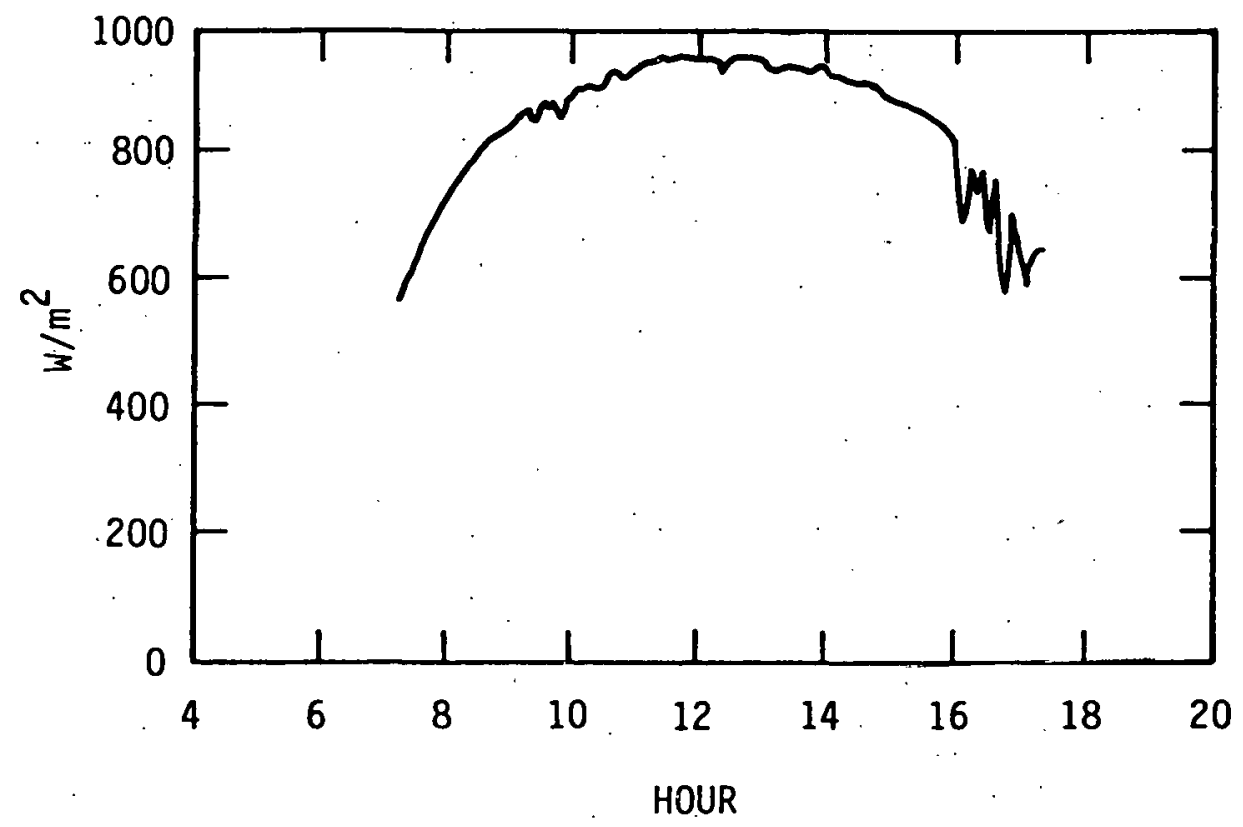

Figure 15. Insolation, 94-1980.

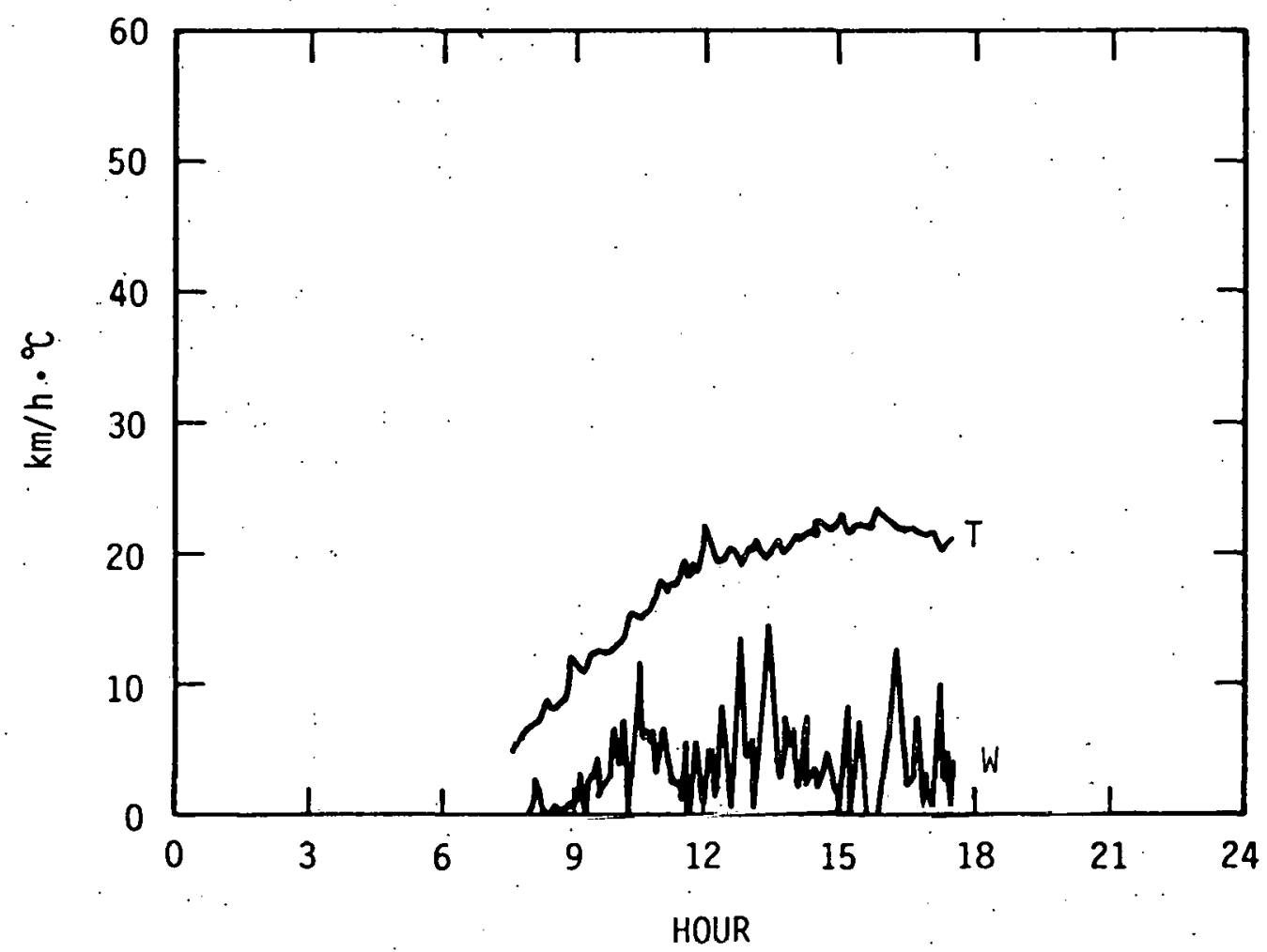

Figure 16. wind speed and Ambient Temperature, 94-1980 


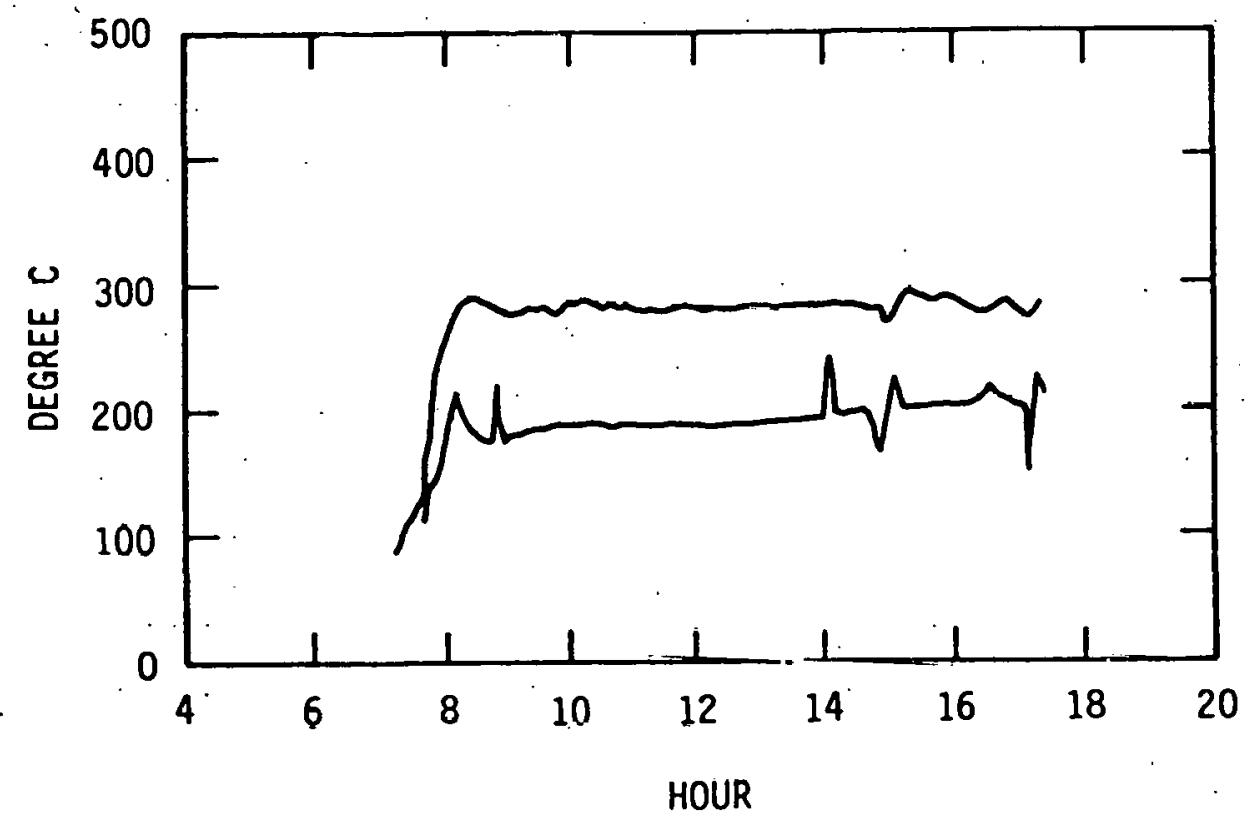

Figure 17. Inlet and outlet Temperatures, 94-1980

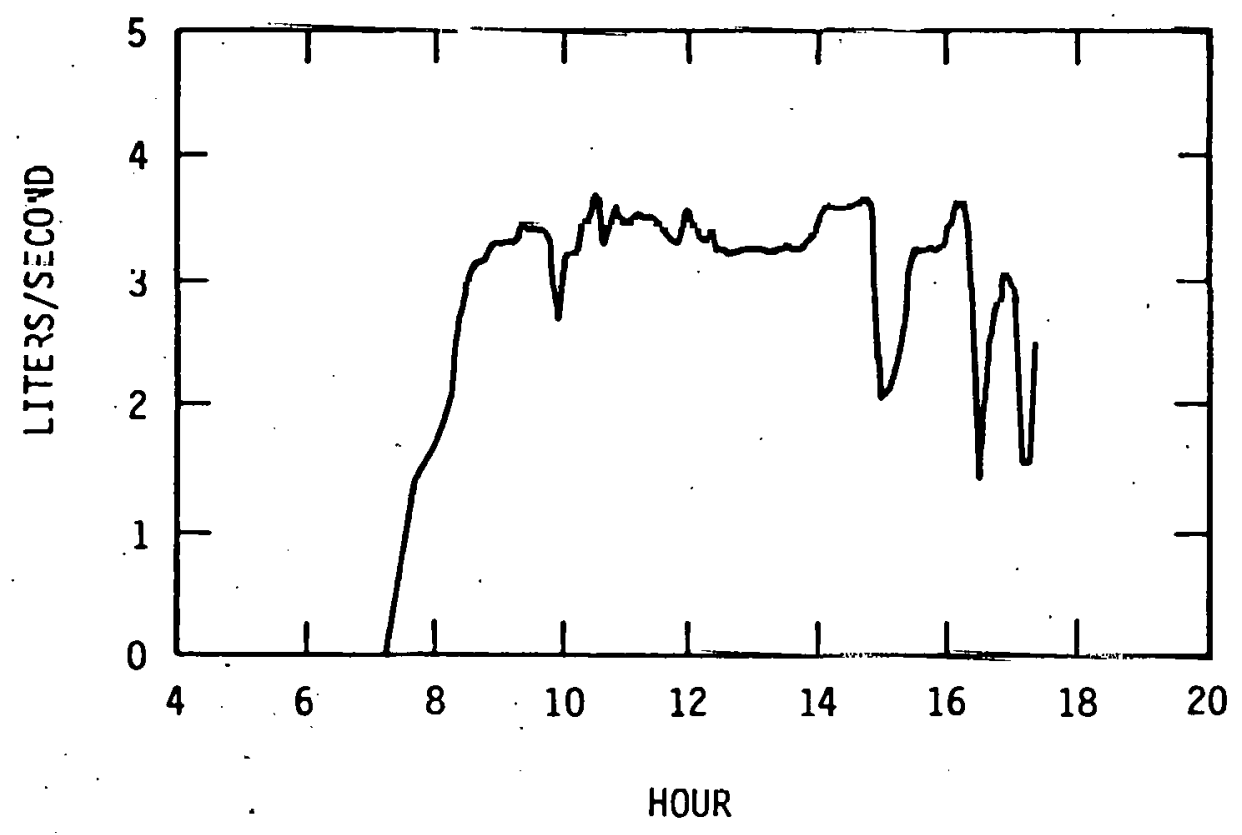

Figure 18. Flow Rate, 94-1980 
PERFORMANCE OF THE SUBSYSTEM AT COOLIDGE, ARIZONA,

25 JUNE AND 4 AND 5 JULY 1980

Solar collector subsystem performance was evaluated on 25 June and 4 and 5 July 1980 (days 177, 186, and 187) to determine solar energy collection efficiency during the period near summer solstice. Average daily subsystem solar energy collection efficiencies for those days were $36.98,36.78$, and 34.48 , respectively. The sustained midday efficiency on the day following collector washing, 25 June, was 408 to 428. Performance was degraded on 4 and 5 July due to intervening dust storms.

\section{Methods}

Collector reflective surfaces and receiver glass covers were washed by a commercial firm prior to commencing the tests. Collector reflector-receiver alignment was checked and adjusted as required to assure optimum performance.

After warmup, Caloria was circulated through the collector subsystem at a flow rate which was controlled to maintain the desired, constant, collertor subsystem outlet temperaturc.

Collector system flow rate was measured with a vortex-sheddingtype meter, temperatures with resistance temperature detector (RTD) sensors, and insolation with a pyrheliometer. Data were recorded at 2-minute intervals.

Collector subsystem efficiency.was computed as the thermal energy gained by Caloria during passage from subsystem inlet to subsystem outlet manifold location divided by the direct normal solar radiation 
impinging on the collector aperture area. Total daily direct normal insolation received during operation was used in the computation of the daily average subsystem efficiency.

Test information is summarized in Table 1 and in the daily performance test presentations.

Table 1

Times Recorded for Performance Test Events

\begin{tabular}{|c|c|c|c|c|}
\hline Event & June 25 & July 4 & Juls & 25 \\
\hline Sunrise & $5: 20 \mathrm{a} \cdot \mathrm{m}$ & $5: 23 \mathrm{a} \cdot \mathrm{m}$ & $5: 23$ & $\mathrm{a} \cdot \mathrm{m}$. \\
\hline $\begin{array}{l}\text { Collectors } \\
\text { Focused }\end{array}$ & $6: 34$ a.m. & $6: 37$ a.m. & $6: 4 b$ & $a \cdot m$. \\
\hline $\begin{array}{l}\text { Flow to } \\
\text { Main } \\
\text { Storage }\end{array}$ & $7: 26$ a.m. & $7: 23$ a.Ill. & $7: 45$ & $a: m$. \\
\hline $\begin{array}{l}\text { Collectors } \\
\text { Defocused }\end{array}$ & $6: 36 \mathrm{p} . \mathrm{m}$ & $6: 36 \mathrm{p} . \mathrm{m}$. & $6: 36$ & $\mathrm{p} \cdot \mathrm{m}$. \\
\hline Sunset & $7: 43$ p.m. & $7: 42$ p.m. & $7: 41$ & $\mathrm{p} \cdot \mathrm{m}$. \\
\hline
\end{tabular}

Conclusions

Days 177 and 187 can be compared to determine the effect of collector washing on collector performance since the solar input was nearly identical for the 2 days. The collectors were very clean on day 177, since they had been washed the day befure. On day 187, the collectors were very dirty again due to dust storms on the preceding days. The suslained midaay collcction efficiency was about $41 \%$ on day 177 and 378 on day 187. The 4-percentage-points difference in collection efficiency represents the improvement that is realized by cleaning. 


\section{$\underline{\text { Day } 177 \text { Results }}$}

Solar collector receiver glass tubes and reflector surfaces were washed and rinsed by a commercial firm on the day prior to this performance test.

The solar collector subsystem gathered energy at a rate of 600 to $800 \mathrm{~kW}(2049$ to $2732 \mathrm{Btu} / \mathrm{h})$ from $8 \mathrm{a} \cdot \mathrm{m}$. to $5 \mathrm{p} \cdot \mathrm{m}$. Solar collector subsystem efficiency was about $40 \%$ during this period, with a computed efficiency of about 428 for an extended time during the period. The average collection efficiency for the entire day was $36.9 \%$.

Day 177 was mostly clear. Winds were 5 to $15 \mathrm{~km} / \mathrm{h}(3$ to $9 \mathrm{mi} / \mathrm{h}$ ) with brief gusts to $20 \mathrm{~km} / \mathrm{h}(12.4 \mathrm{mi} / \mathrm{h})$. The ambient temperature was over $40^{\circ} \mathrm{C}\left(104^{\circ} \mathrm{F}\right)$ during much of the test period.

The collector subsystem outlet temperature was maintained at about $284^{\circ} \mathrm{C}\left(543^{\circ} \mathrm{F}\right)$ during the test period. Inlet oil temperature was $184^{\circ}$ to $200^{\circ} \mathrm{C}\left(363^{\circ}\right.$ to $\left.392^{\circ} \mathrm{F}\right)$. Collector subsystem Caloria flow rate was 3 to $4 \mathrm{l} / \mathrm{s}$ ( 47 to $63 \mathrm{gal} / \mathrm{min}$ ) during most of the test but varied considerably, becoming about $4.5 \mathrm{l} / \mathrm{s}(71 \mathrm{gal} / \mathrm{min})$ late in the test period. 


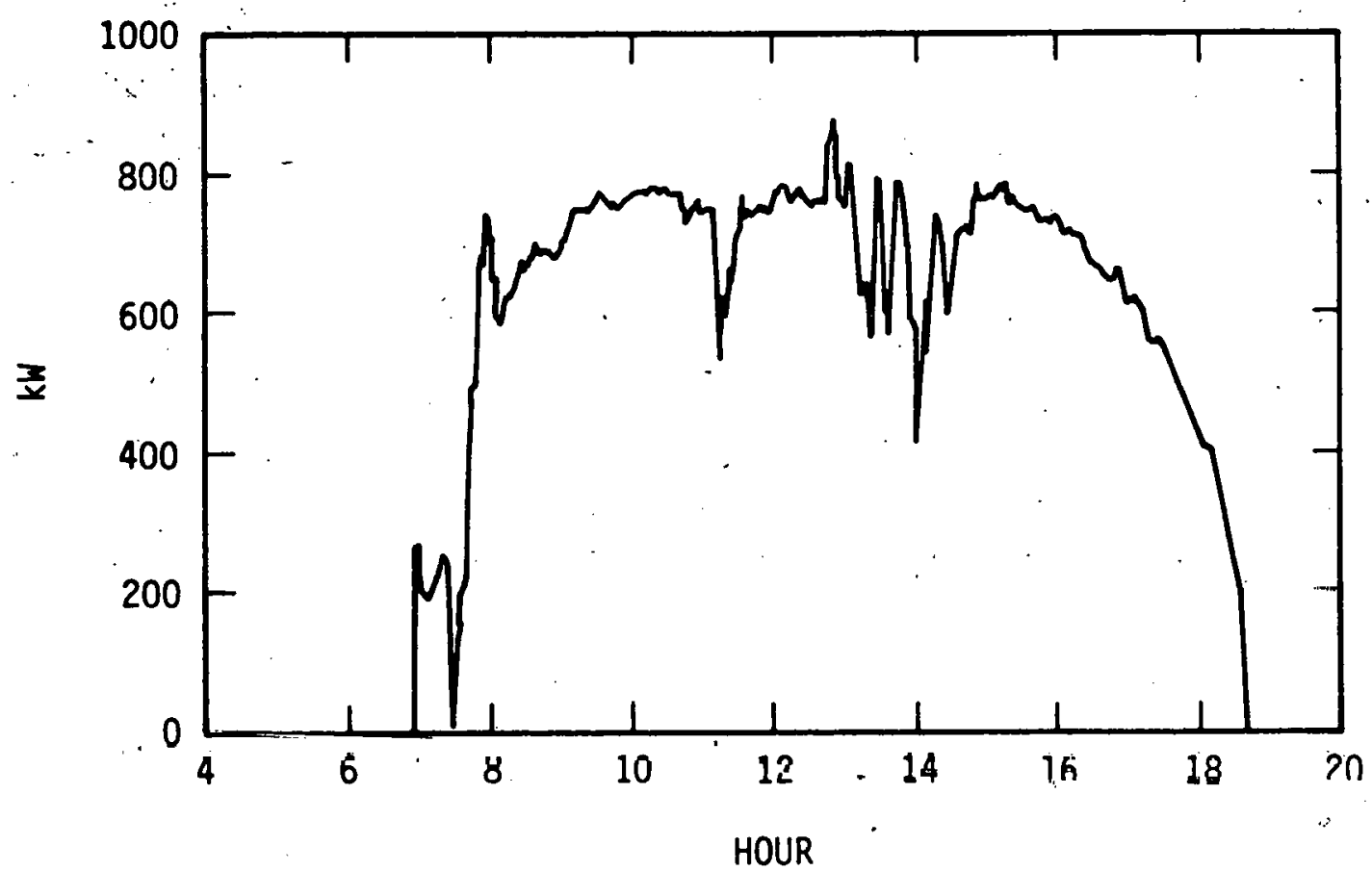

Figure 1. Collected Power, 177-1980,

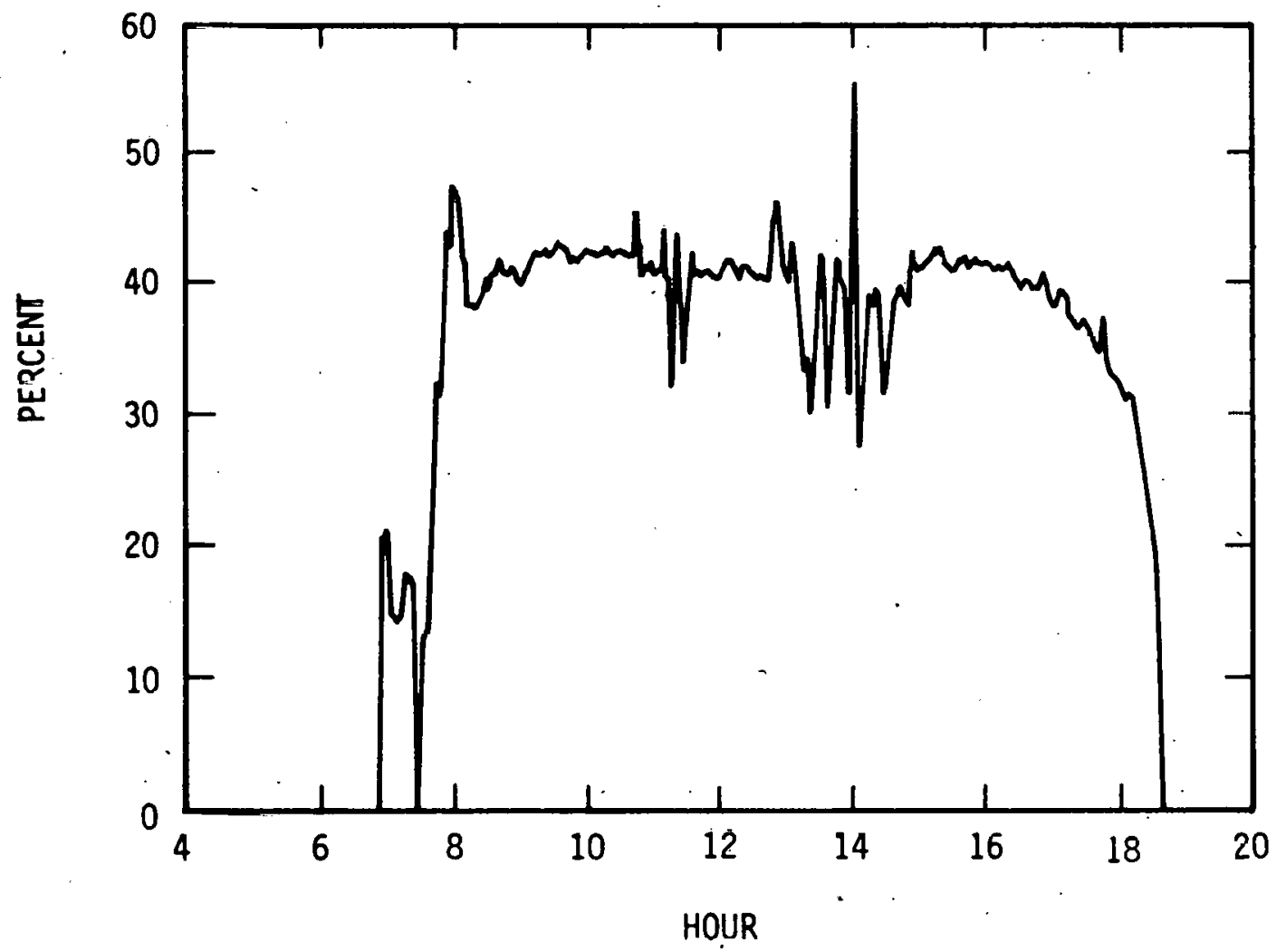

Figure 2. Efficiency, 177-1980 


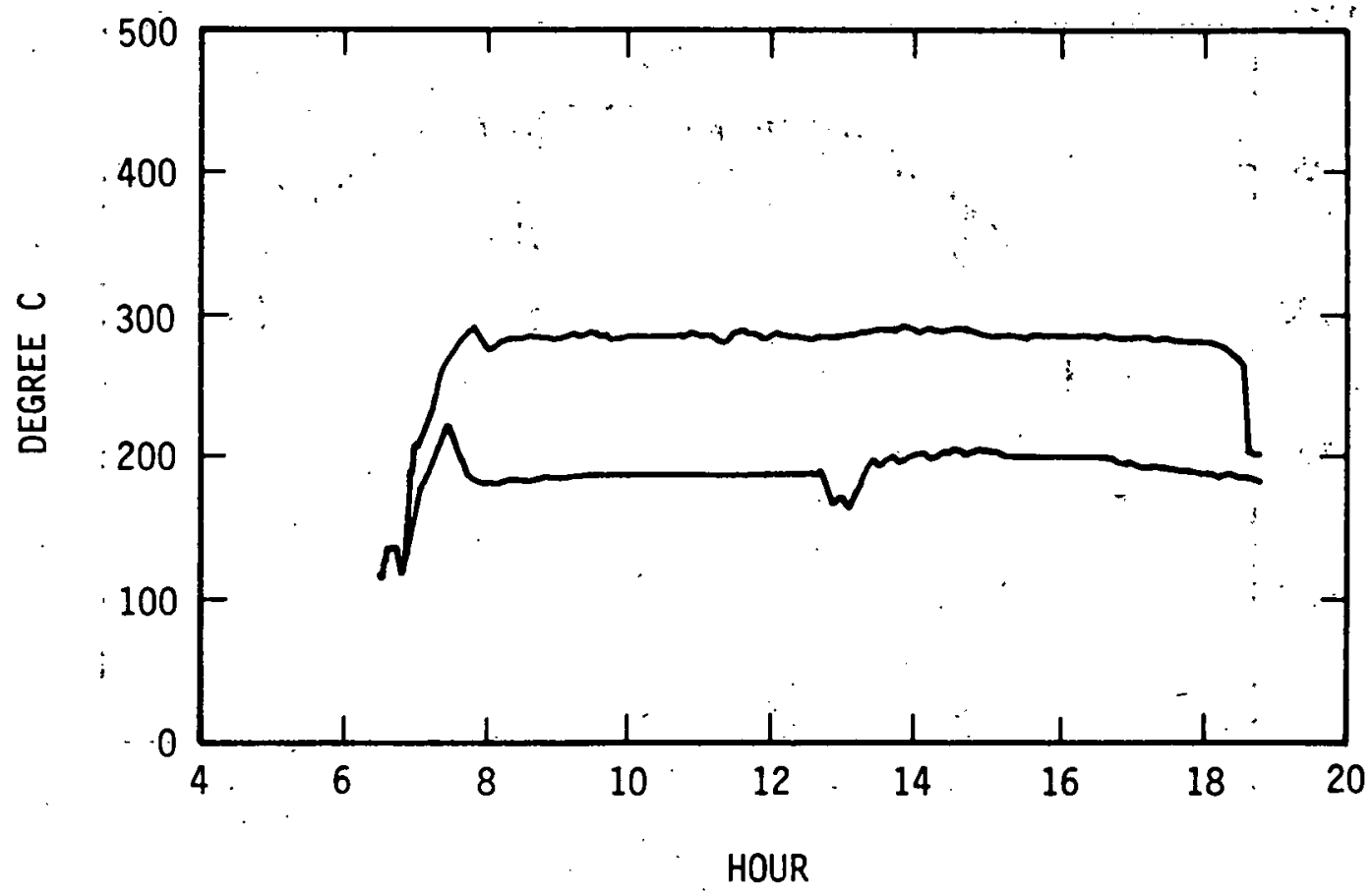

Figure 3. Inlet and outlet. Temperatures, $177-1980$

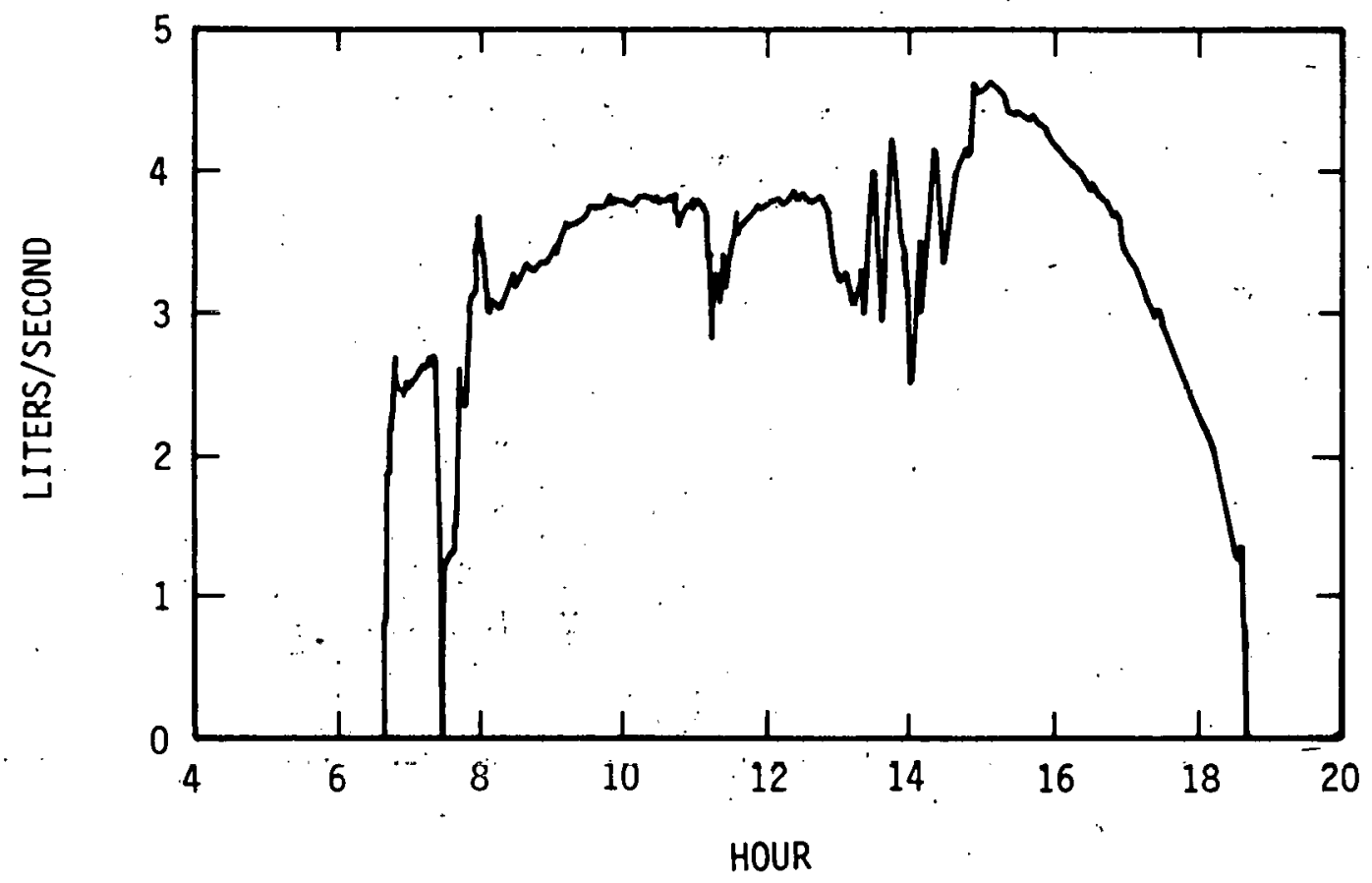

Figure 4. Flow Rate, 177-1980 


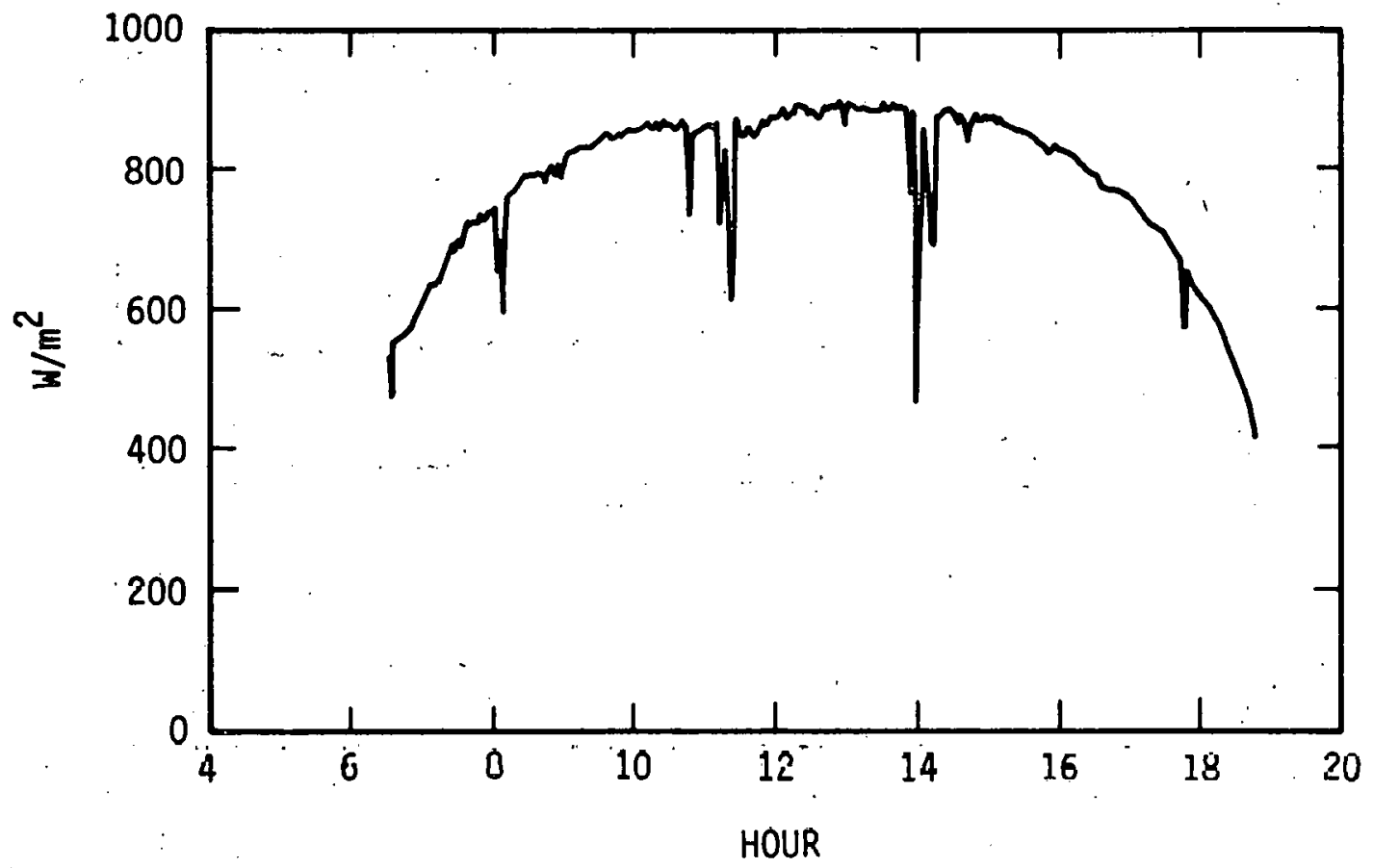

Figure 5. Insolation, 177-1980

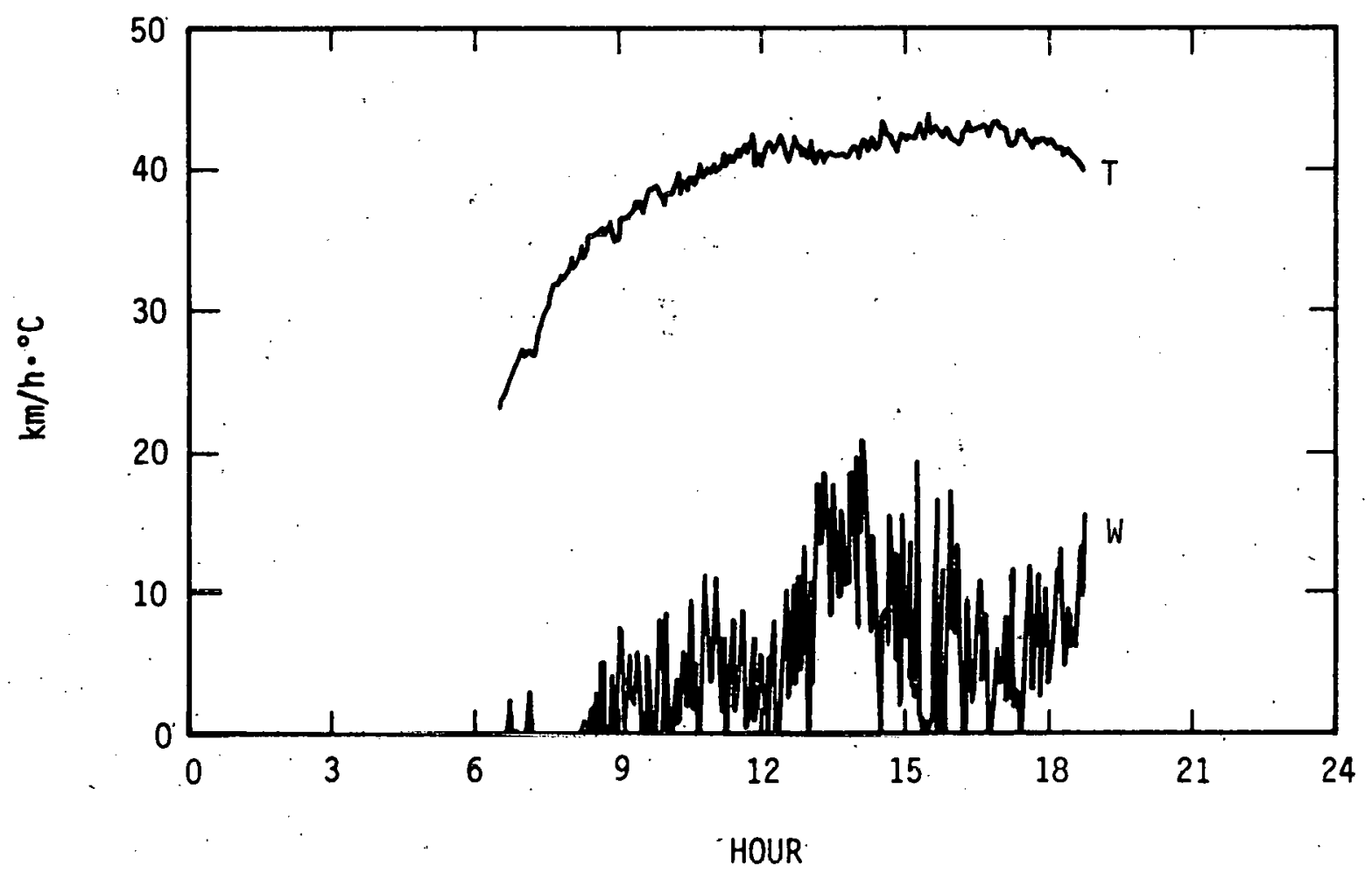

Figure 6. Wind Speed and Ambient Temperature, $177-1980$ 


\section{Day 186 Results}

Stormy weather, including high winds and blowing dust, occurred on the intervening days between 25 June and 4 July collector subsystem performance evaluation tests. Collectors were stowed when blowing dust was most pronounced, but reflector surfaces were dirtied somewhat. The receiver cover tubes were wiped prior to day 186 tests.

Thermal energy was collected at a rate of about $800 \mathrm{~kW}$ $(1,000 \mathrm{hp})$. The collector subsystem efficiency varied from about $38 \%$ to over $42 \%$ during the test period with brief periods of higher efficiency. The average solar collector subsystem efficiency throughout the day was 36.78 .

Day 186, 5 July, was clear with good insolation, about $960 \mathrm{~W} / \mathrm{m}^{2}$ $\left(304 \mathrm{Btu} / \mathrm{ft}^{2} \cdot \mathrm{h}\right)$ at noon. The ambient temperature was about $35^{\circ} \mathrm{C}$ $\left(95^{\circ} \mathrm{F}\right)$ at $9 \mathrm{a} . \mathrm{m} . ;$ the high temperature was about $44^{\circ} \mathrm{C}\left(111^{\circ} \mathrm{F}\right)$ : Wind speeds varied around $10 \mathrm{~km} / \mathrm{h}(6 \mathrm{mi} / \mathrm{h})$ in the morning, becoming somewhat higher in the afternoon.

Collector subsystem outlet oil temperature was maintained at about $264^{\circ} \mathrm{C}\left(507^{\circ} \mathrm{F}\right)$ early in the morning but dropped gradually to about $253^{\circ} \mathrm{C}\left(487^{\circ} \mathrm{F}\right)$ by $3 \mathrm{p} . \mathrm{m}$. The inlet temperature varied from about $283^{\circ}$ to $289^{\circ} \mathrm{C}\left(541^{\circ}\right.$ to $\left.552^{\circ} \mathrm{F}\right)$ during the test. The collector subsystem Caloria flow rate varied greatly during the day as the control system attempted to maintain system outlet temperature at the desired, constanl level. 'l'he flow rate climbed from about $3.5 \mathrm{l} / \mathrm{s}$ ( $46 \mathrm{gal} / \mathrm{min})$ at $8 \mathrm{a} . \mathrm{m}$. to $5.5 \mathrm{l} / \mathrm{s}(73 \mathrm{gal} / \mathrm{min})$ at $10 \mathrm{a} . \mathrm{m}$. and then varied from about $5.3 \mathrm{l} / \mathrm{s}(70 \mathrm{gal} / \mathrm{min})$ to $6.3 \mathrm{l} / \mathrm{s}(83 \mathrm{gal} / \mathrm{min})$ during the rest of the test period. 


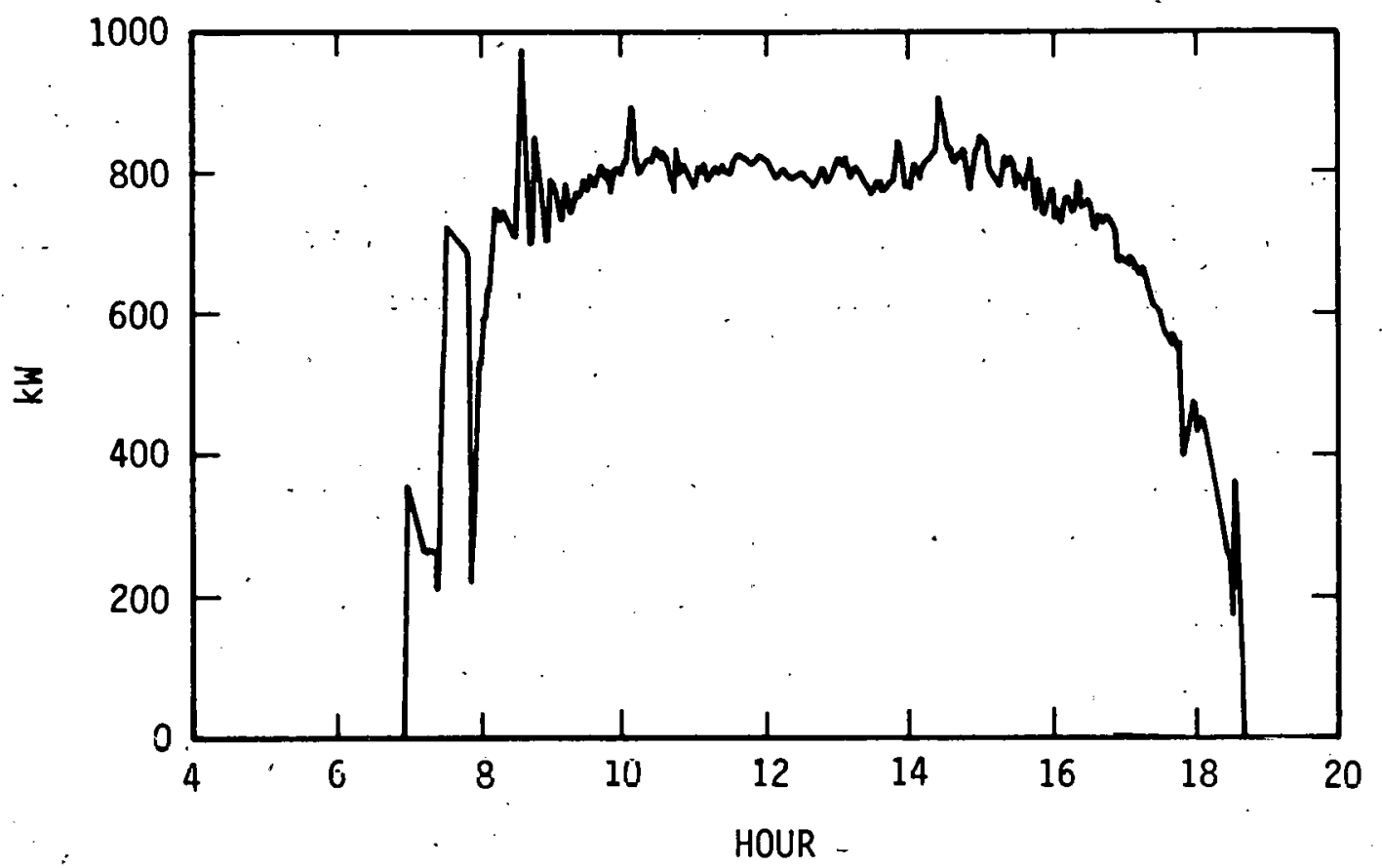

Figure 7. Collected Power, 186-1980

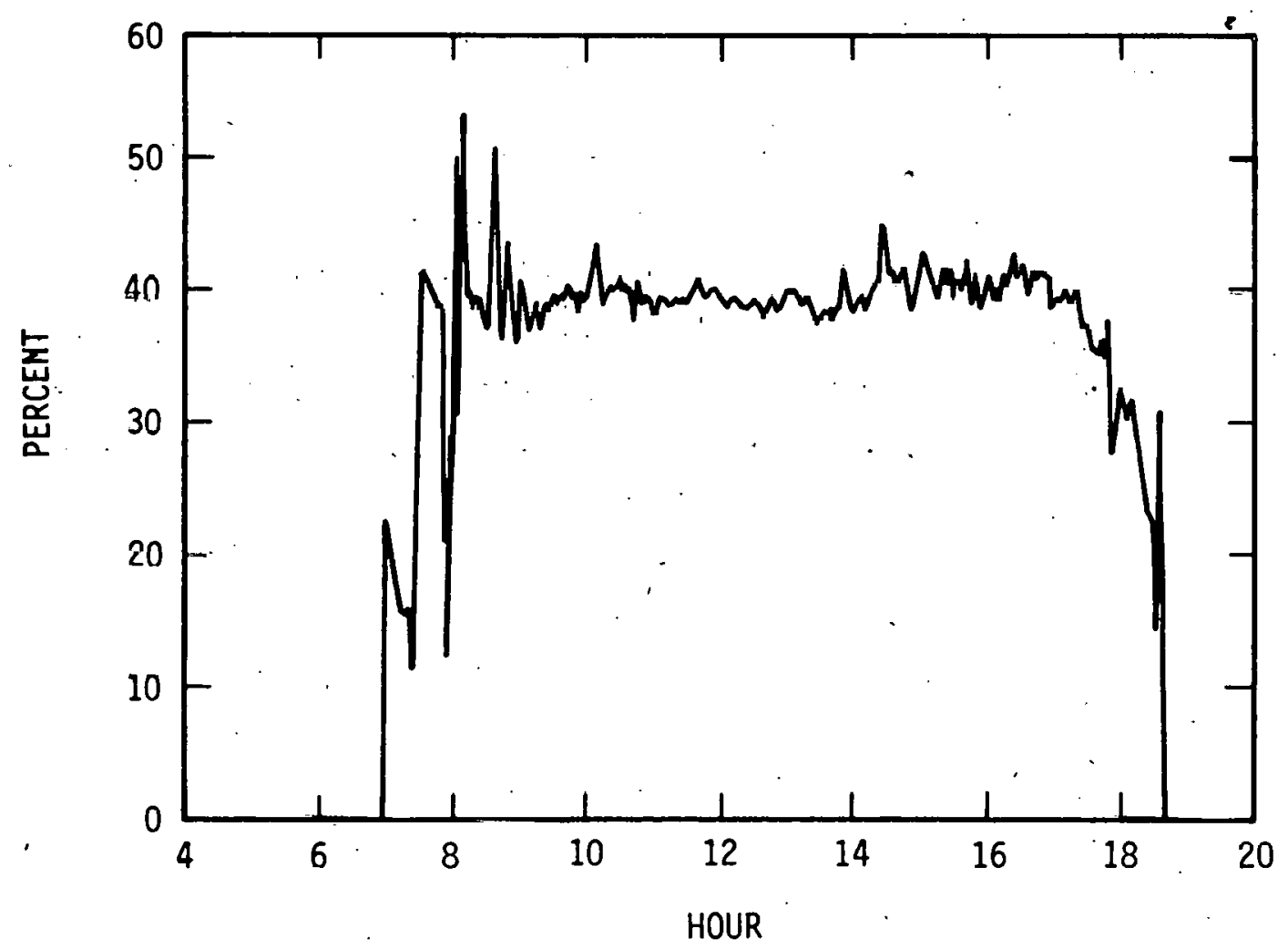

Figure 8. Efficiency, 186-1980. 


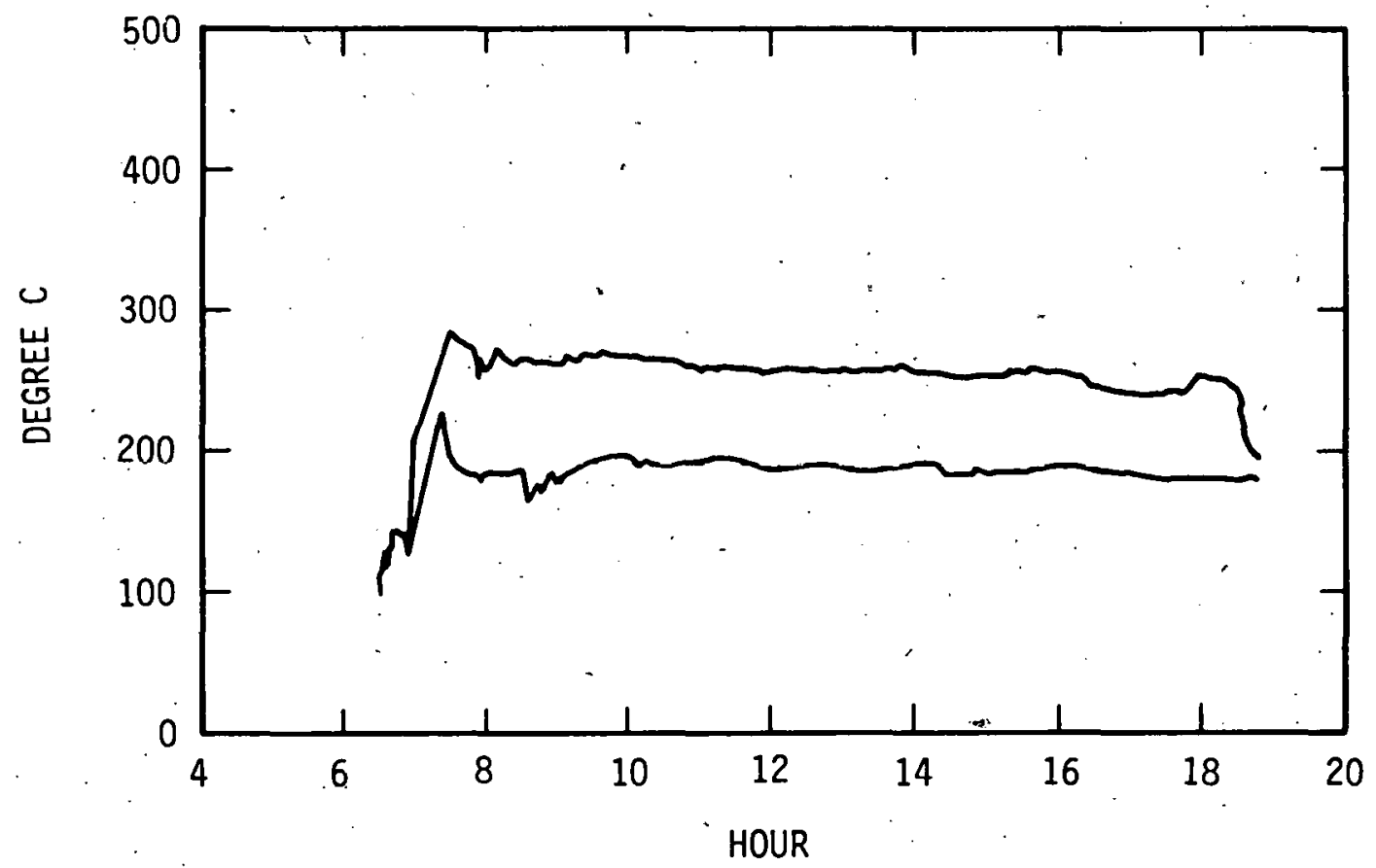

Figure 9. Inlet and Outlet Temperatures; $186-1980$ 


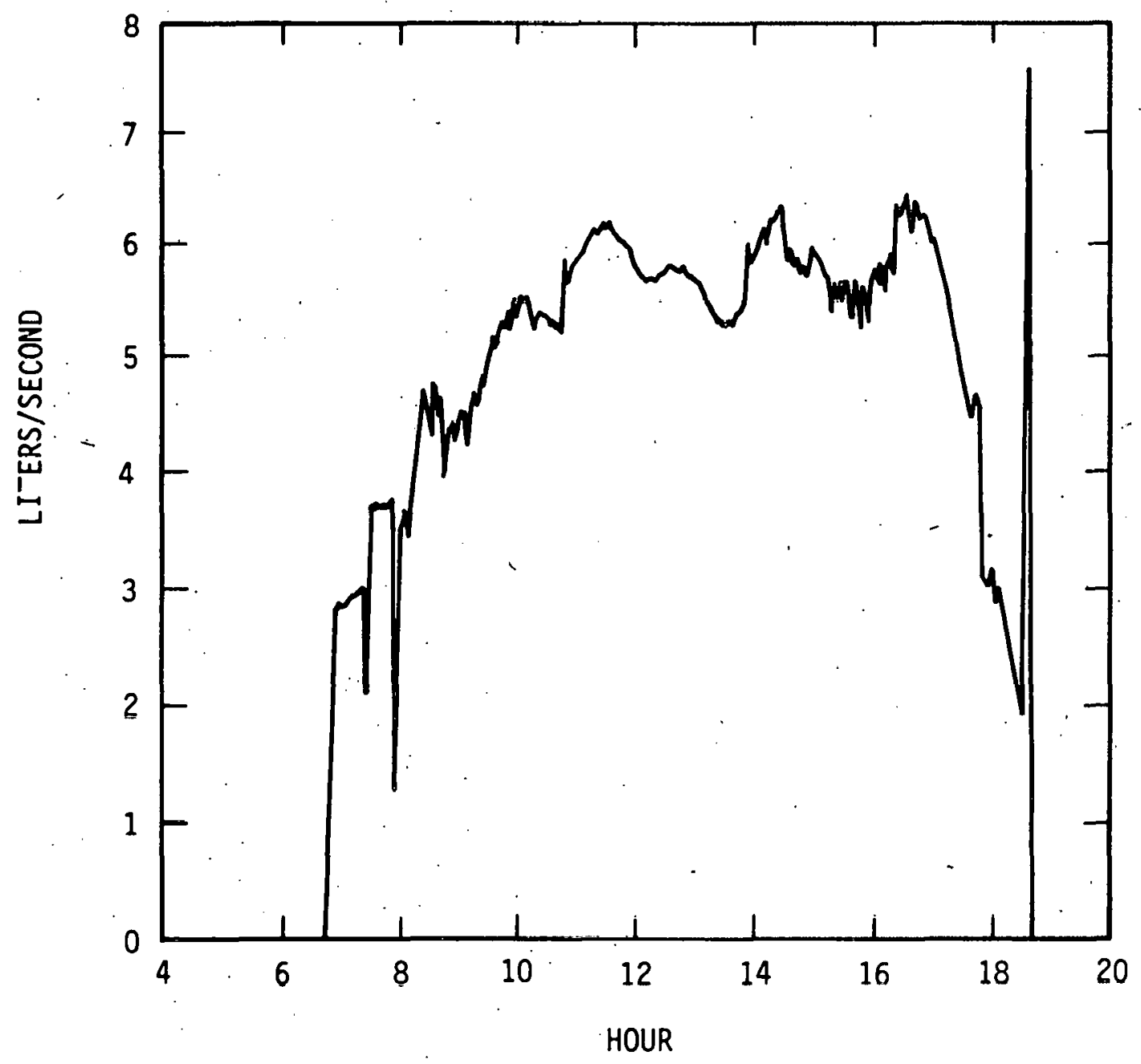

Figure 10. Flow Rate, 186-1980 


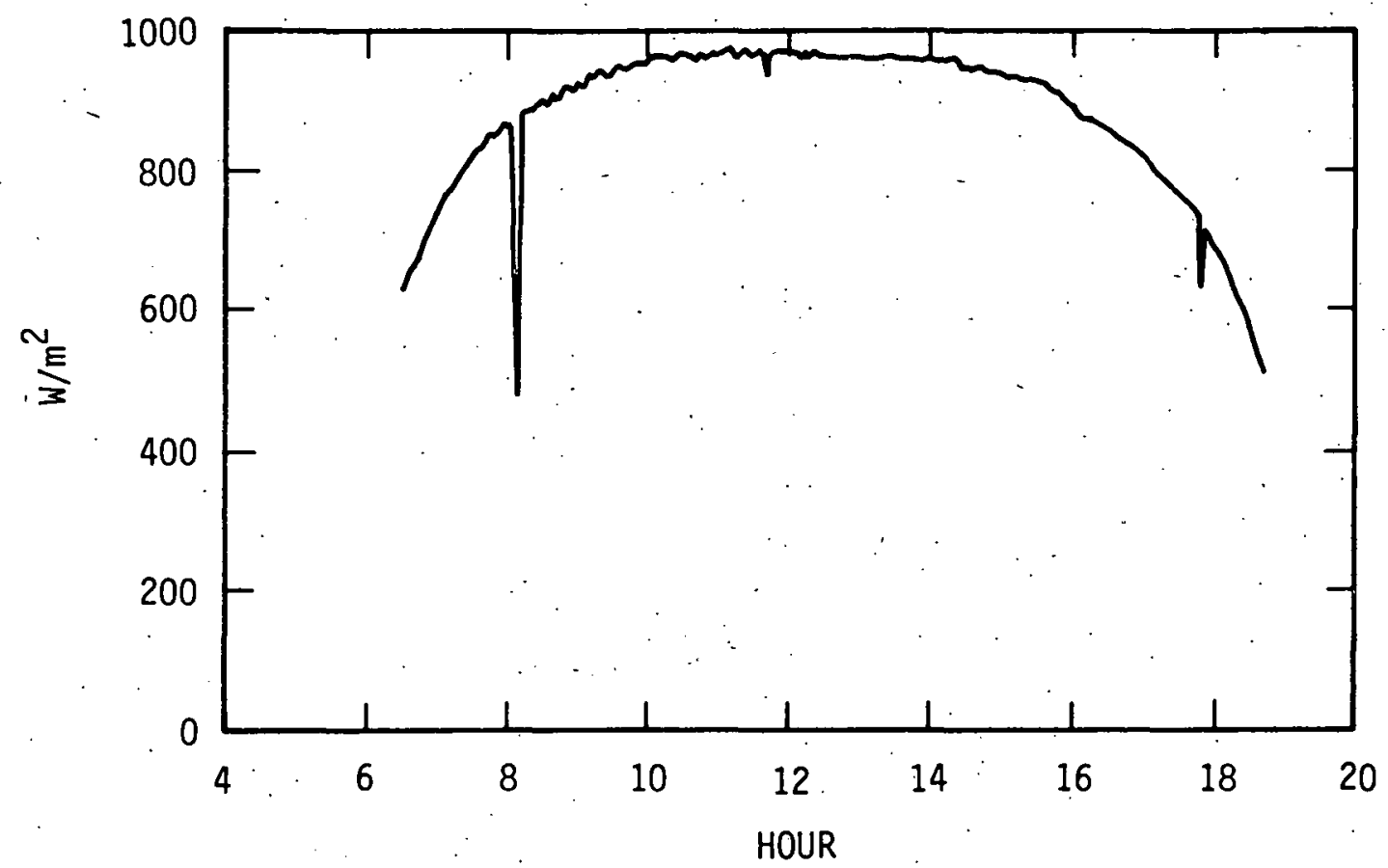

Figure 11. Insolation, 186-1980

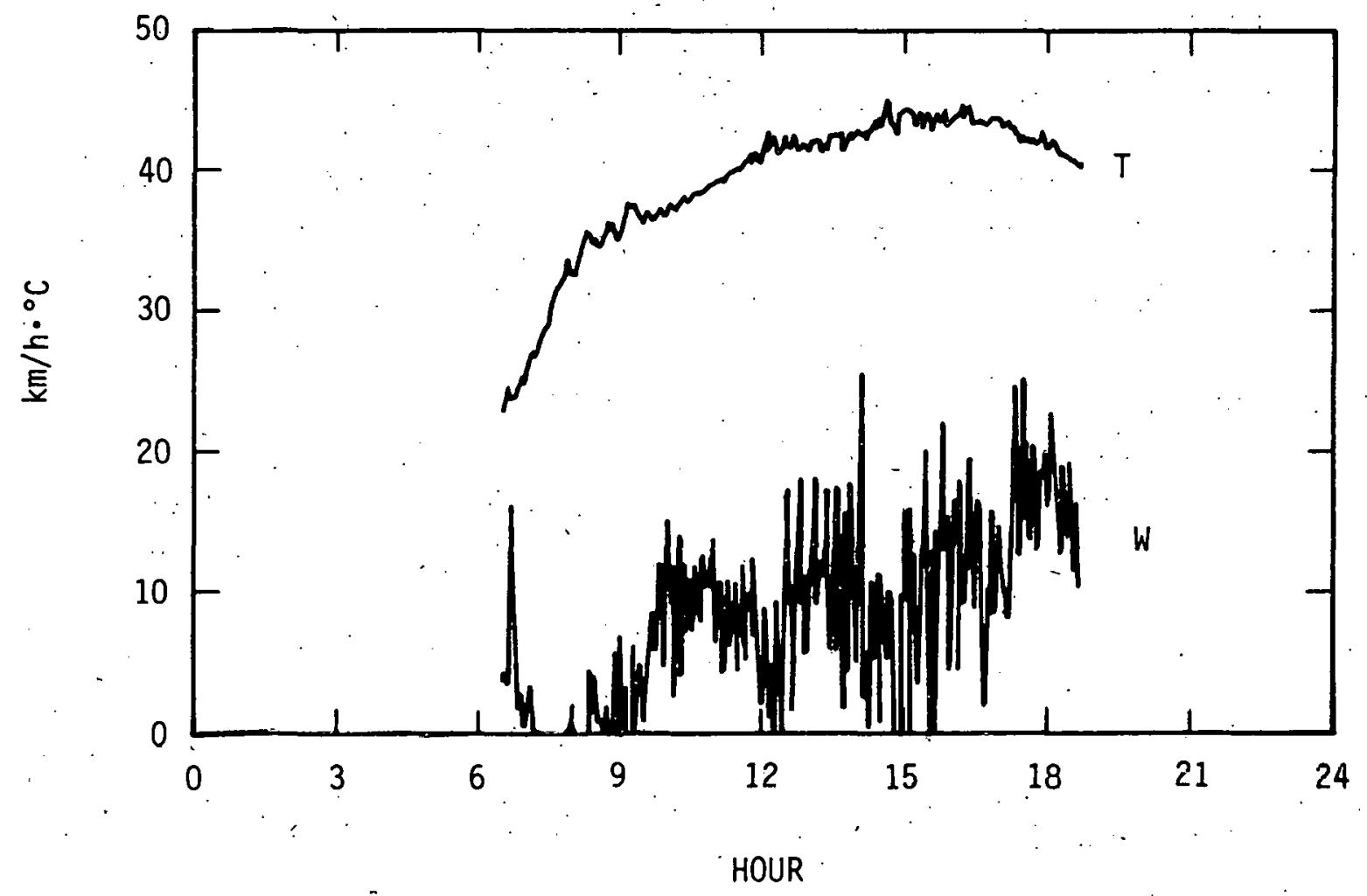

Figure 12. Wind speed and Ambient Temperature, 186-1980 


\section{Day 187 Results}

Thermal energy was collected at a rate of about 650 to $700 \mathrm{~kW}$ ( 87.2 to $939 \mathrm{hp}$ ) on day 187 ( $5 \mathrm{July})$. Collector subsystem efficiency was computed to be from about 368 to $38 \%$ during the $8 \mathrm{a} . \mathrm{m}$. to $5 \mathrm{p} . \mathrm{m}$. period. The average collector subsystem efficiency for the entire day was. 34.48 .

Day 187 was mostly clear. However, morning insolation was somewhat reduced, and a brief cloudy period occurred at about 12:30 p.m. Ambient daytime temperatures were about $34^{\circ} \mathrm{C}\left(93^{\circ} \mathrm{F}\right)$ at 9 a.m. and near $40^{\circ} \mathrm{C}\left(104^{\circ} \mathrm{F}\right)$ in the afternoon. Wind speeds were highly variable with a representativè measuiement of $10 \mathrm{~km} / \mathrm{h}(6 \mathrm{mi} / \mathrm{h})$.

Collector subsystem outlet temperature was maintained at about $276^{\circ} \mathrm{C}\left(529^{\circ} \mathrm{F}\right)$ during the performance test. Inlet Caloria temperature was $185^{\circ}$ to $200^{\circ} \mathrm{C}\left(365^{\circ}\right.$ to $\left.392^{\circ} \mathrm{F}\right)$. Collector subsystem flow rate ranged from about $3.4 \mathrm{l} / \mathrm{s}(45 \mathrm{gal} / \mathrm{min}$ ) early in the test day to nearly $5 \mathrm{l} / \mathrm{s}(66 \mathrm{gal} / \mathrm{min})$ in the afternoon in order to control system fluid outlet temperature. 


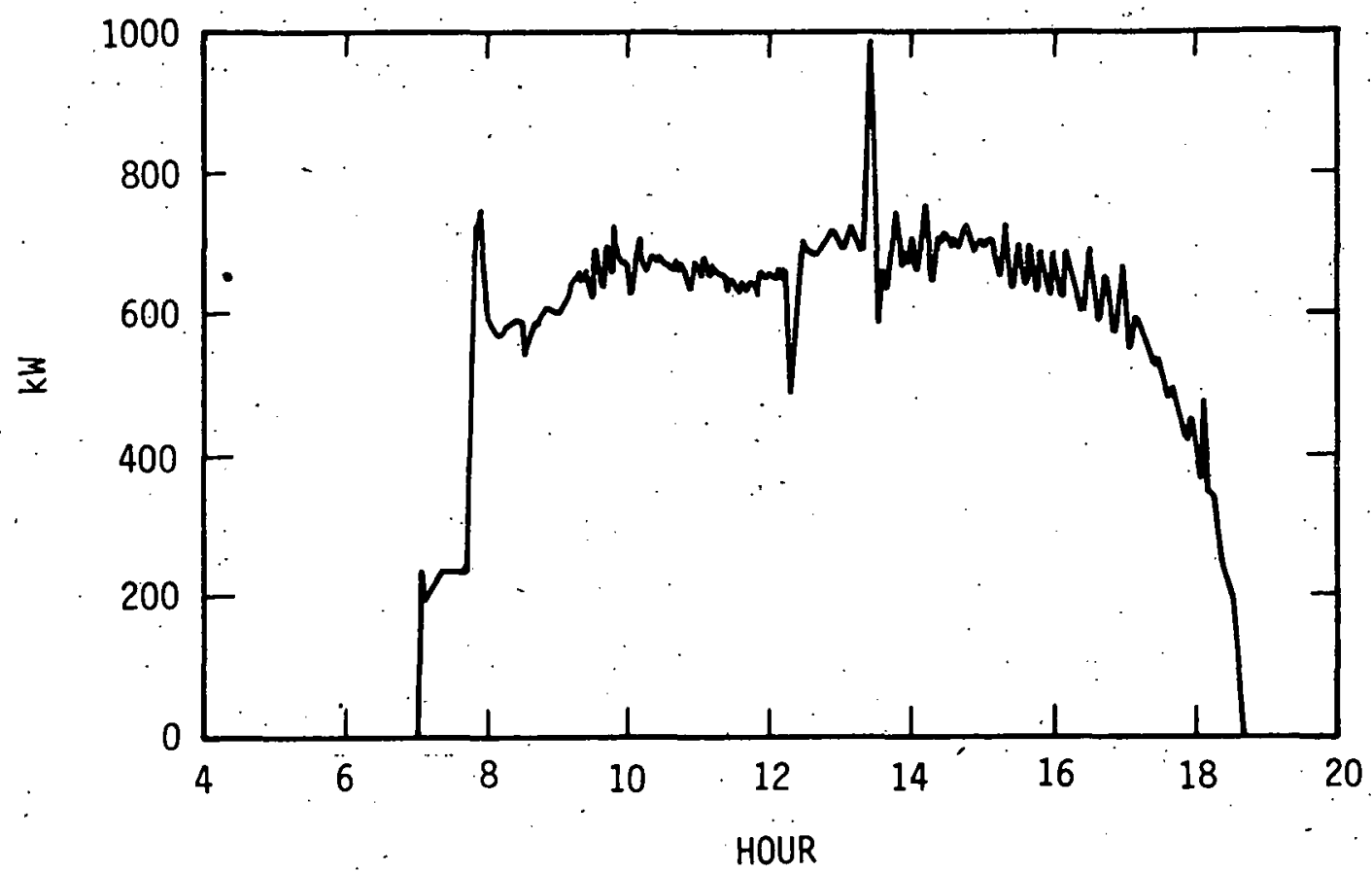

Figure 13. Collected Power, 187-1980

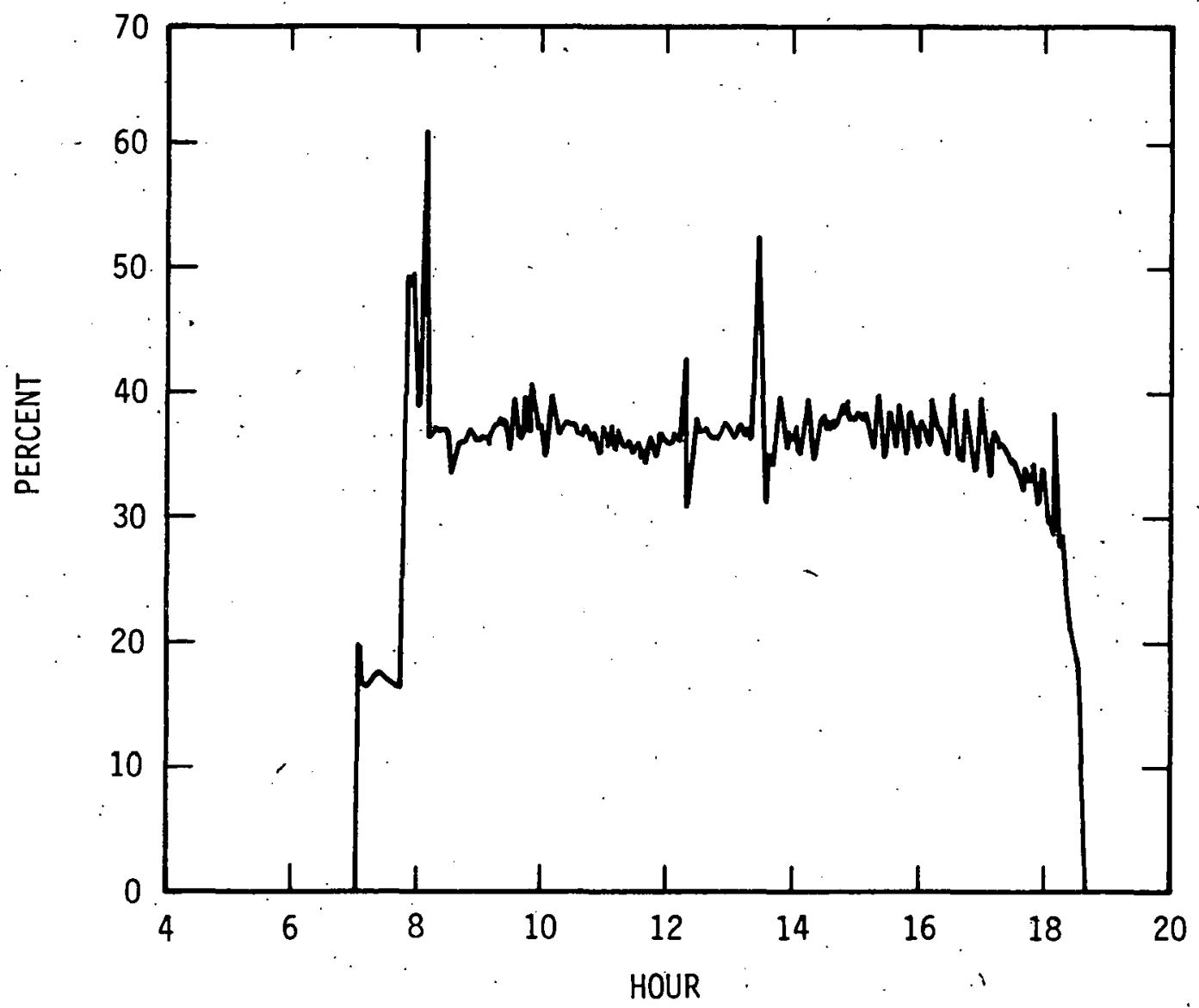

Figure 14. Efficiency, 187-1980 


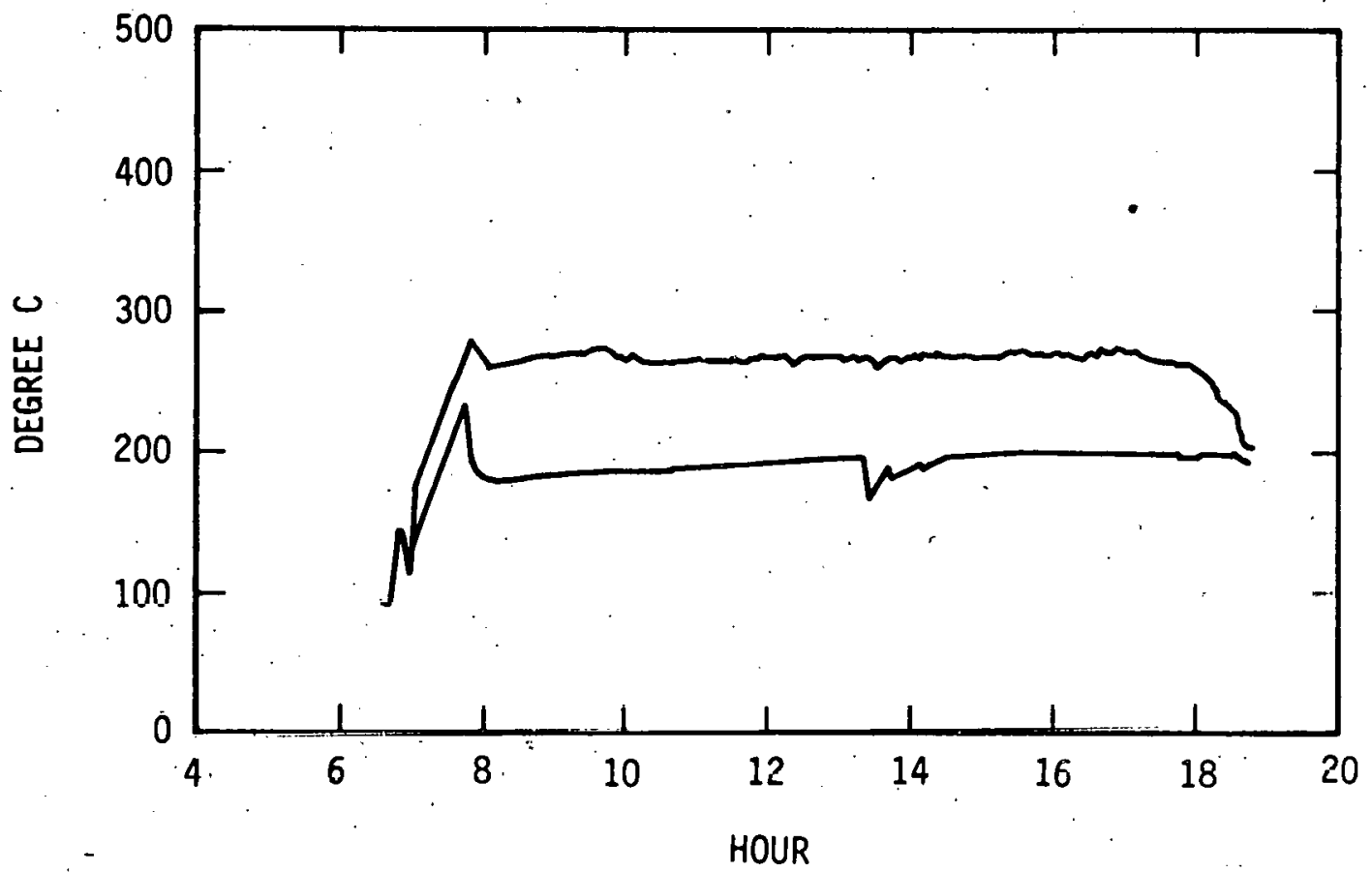

Figure 15. Inlet and Outlet Temperatures, 187-1980

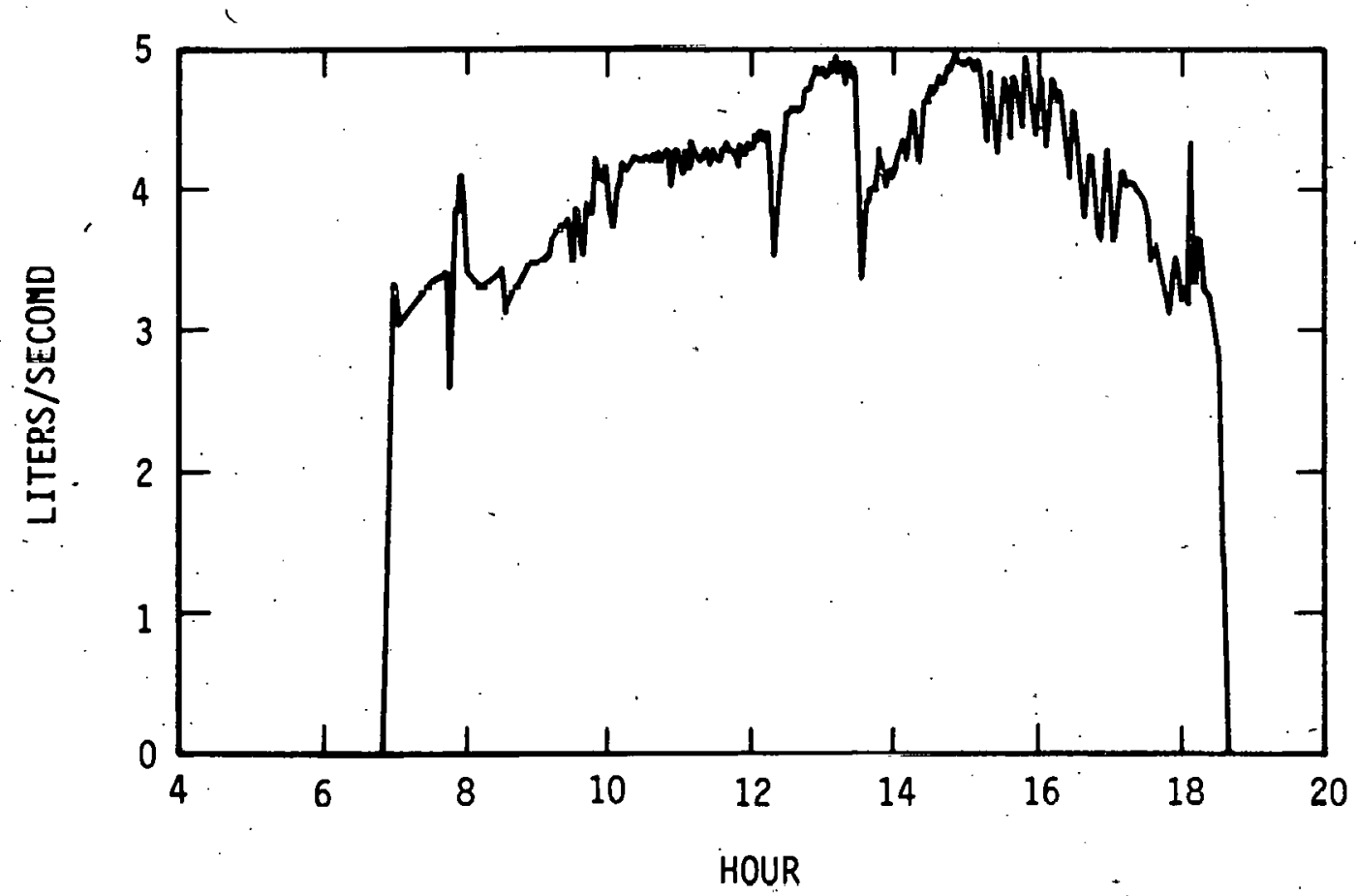

Figure 16. Flow Rate, 187-1980 


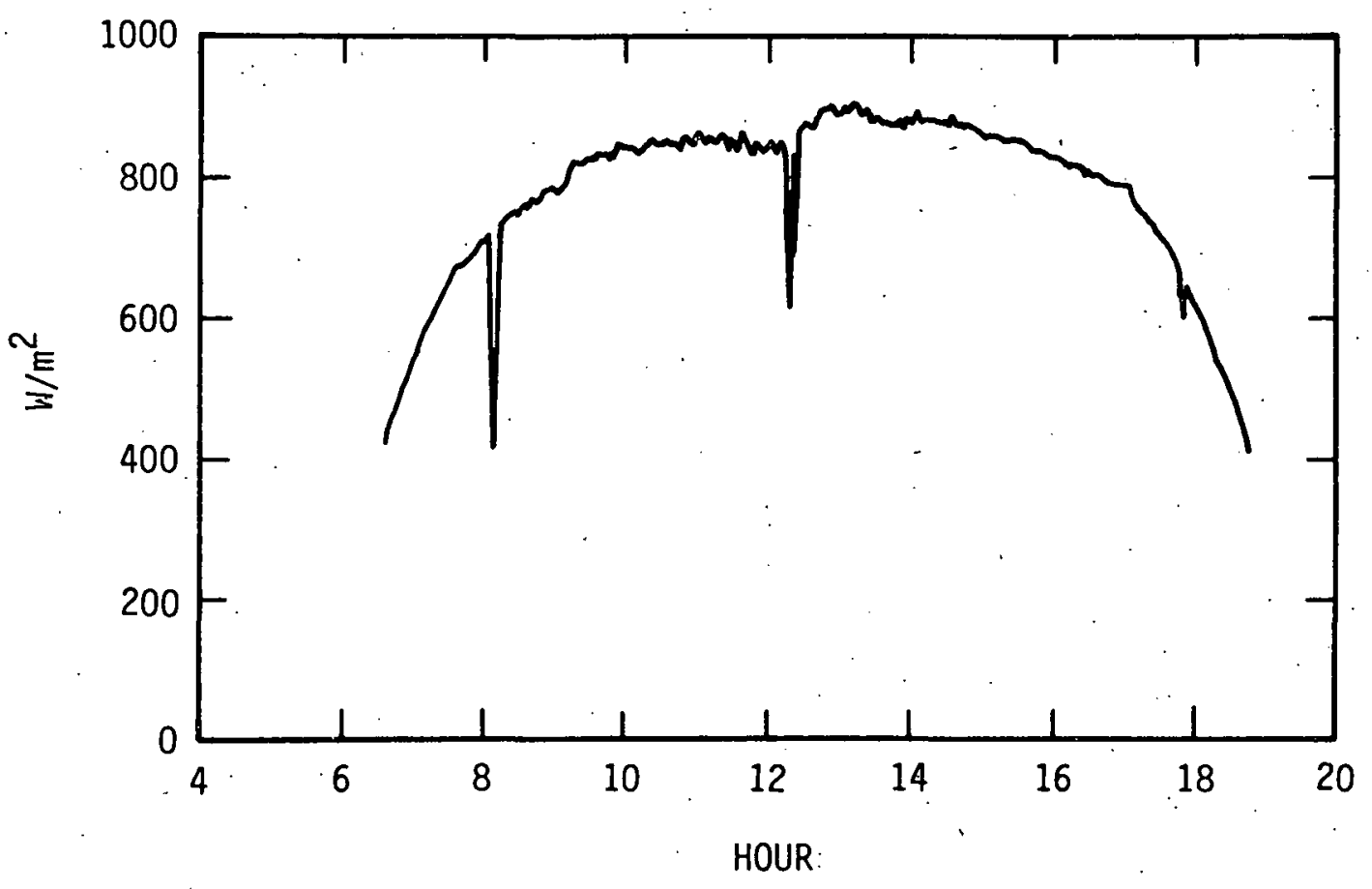

Figure 17. Insolation, 187-1980

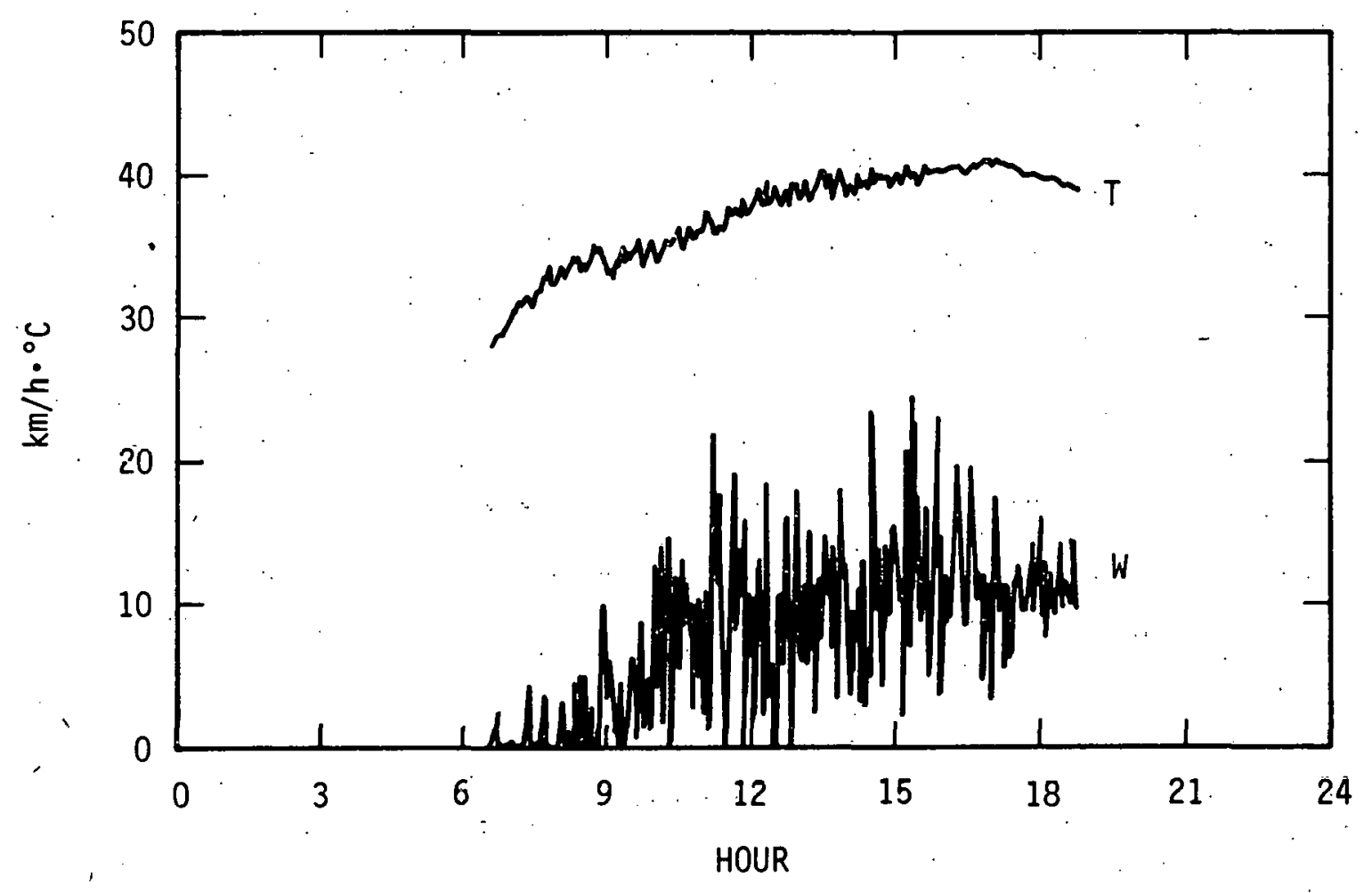

Figure 18. Wind Speed and Ambient Temperature, 187-1980 
SOLAR COLLECTOR SUBSYSTEM PERFORMANCE PREDICTIONS

A comparison was made between the collector field subsystem performance at the Coolidge Solar Irrigation Facility and the predicted collector field performance, using a systems analysis model and Phoenix, Aṛizona, typical meteorological year (TMY) weather data. The systems analysis model calculates field performance on an hourly basis for an entire year and takes into account such effects as field thermal losses, end losses, shadowing, incidence angle effects, and reduced reflectance of dirty collectors. The equations for collector performance (normal. incidence) and incidence angle modifier were derived from data taken at the Collector Module Test Facility in Albuquerque, New Mexico. The equation for field thermal losses was derived from tests performed at the Coolidge facility. The results from the model indicate an annual average collector field efficiency of about 208 at design temperature with daily average efficiencies reaching $30 \%$ to $32 \%$ around the summer solstice. If the collectors could be continuously cleaned, the model indicates an annual average efficiency of about $24 \%$ with daily average efficiencies of 358 to $37 \%$ around the summer solstice. Actual collector field efficiencies measured at the Coolidge facility with recently cleaned collectors on 25 June and 4 and 5 July 1980 were $36.98,36.78$, and 34.48 , respectively. These daily average efficiencies agree very closely with the 358 to 378 predicted for a clean field near the summer solstice.

Figurcs 1 and 2 are plots of calculated collector field subsystem thermal output and efficiency, respectively, for dirty collectors. Figures 3 and 4 are plots of calculated collector field subsystem thermal output and efficiency, respectively, for continuously cleaned collectors. 
EN ERGY AVAILABLE PHX

ONE AXIS TRACKING

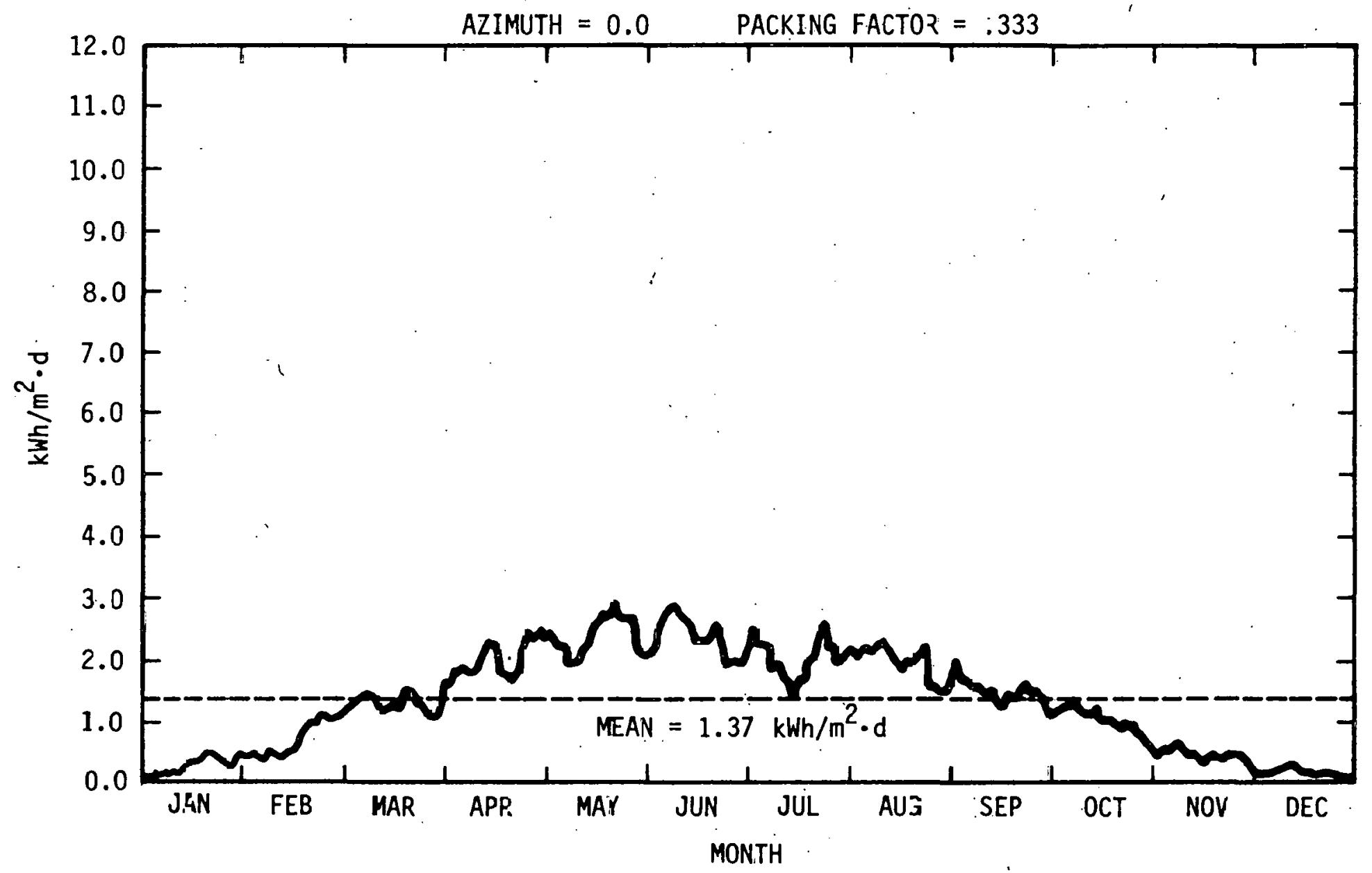

Figure 1: Calculated Collector Field Subsystem Thermal output: Dirty Collectors 


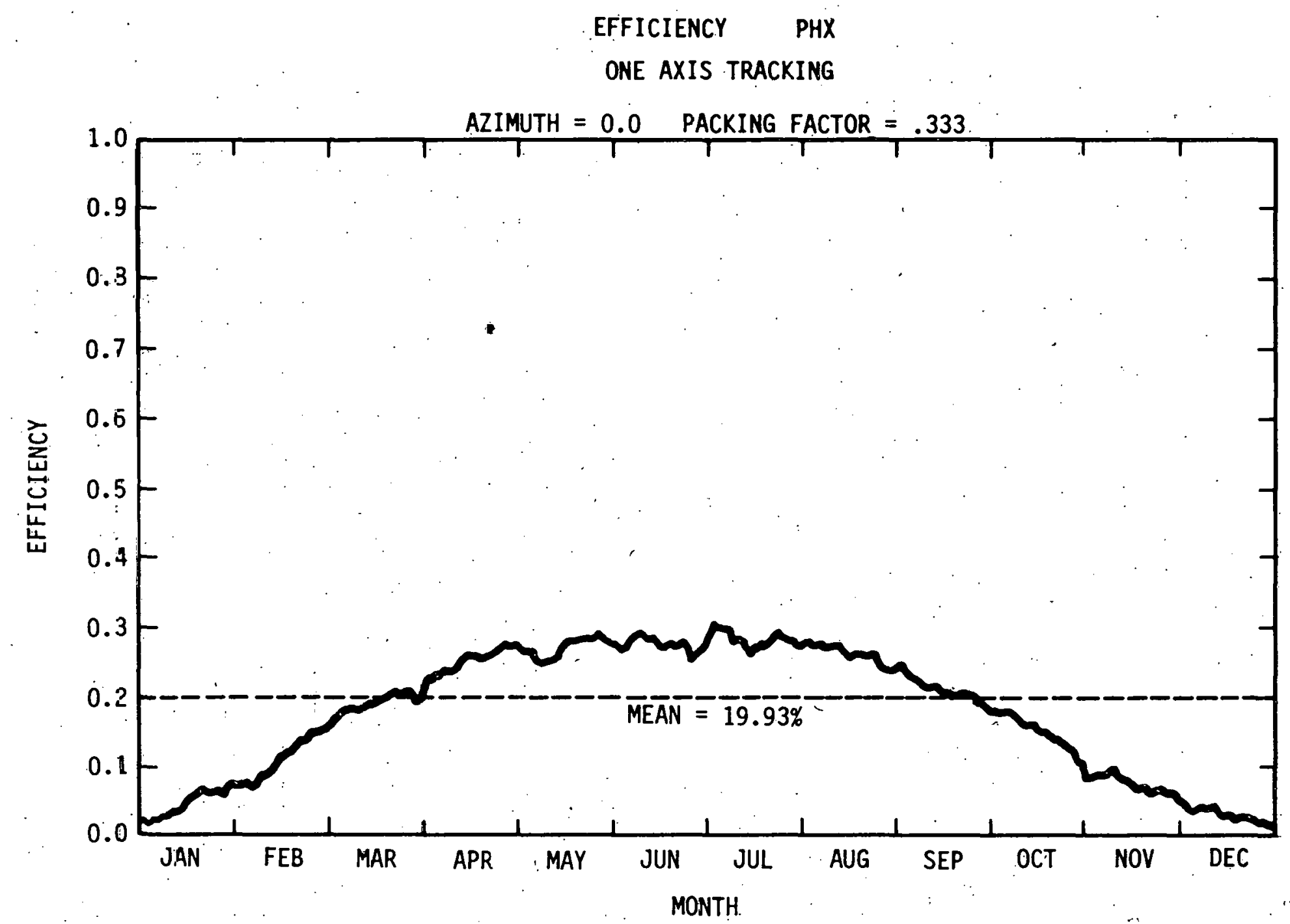

Figure 2. Calculated Collector Field Subsystem Efficiency: Dirty Collectors 


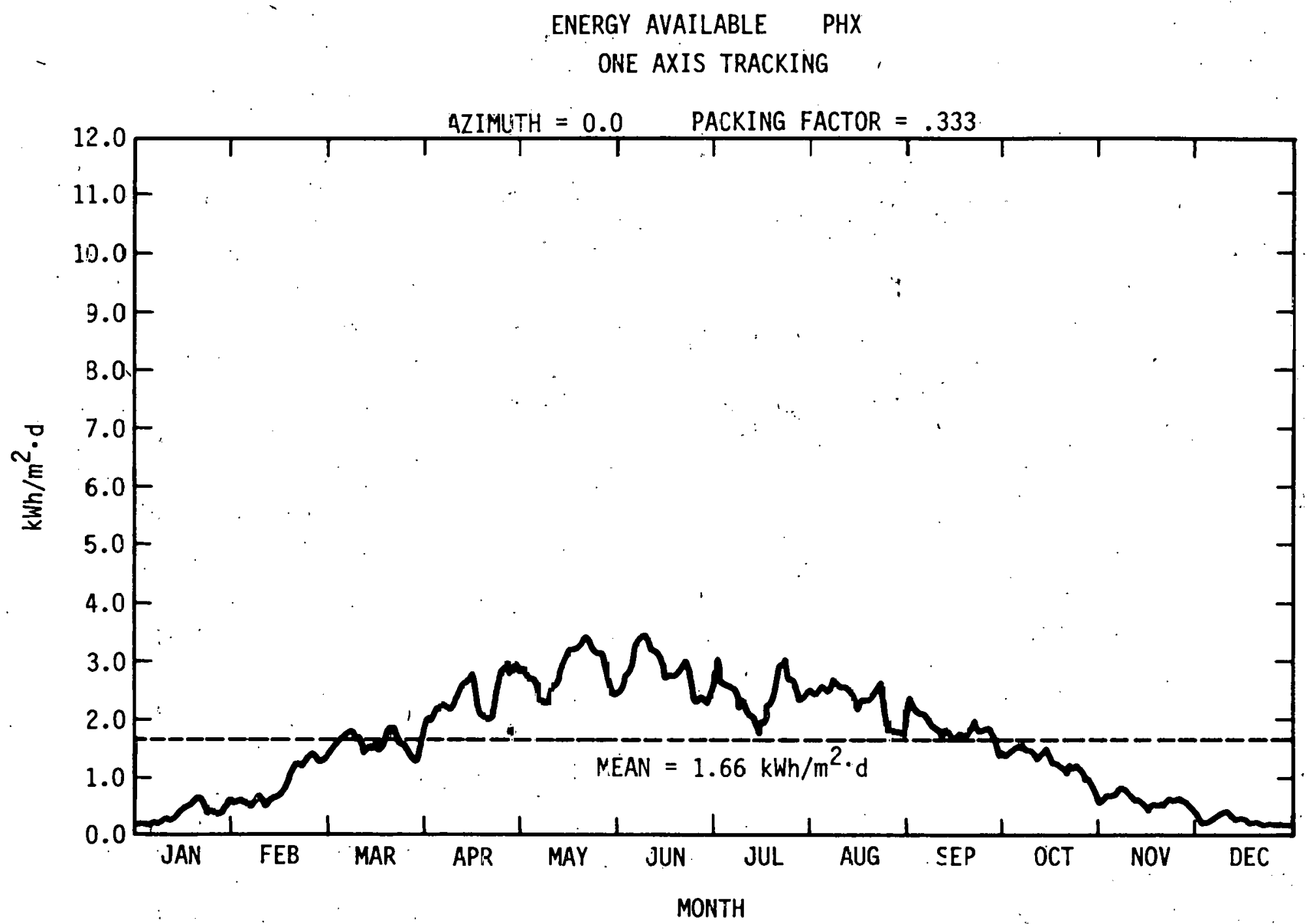

Figure 3. Calculated collector Field Subsystem Thermal output: Continuausly cleaned Collectors 


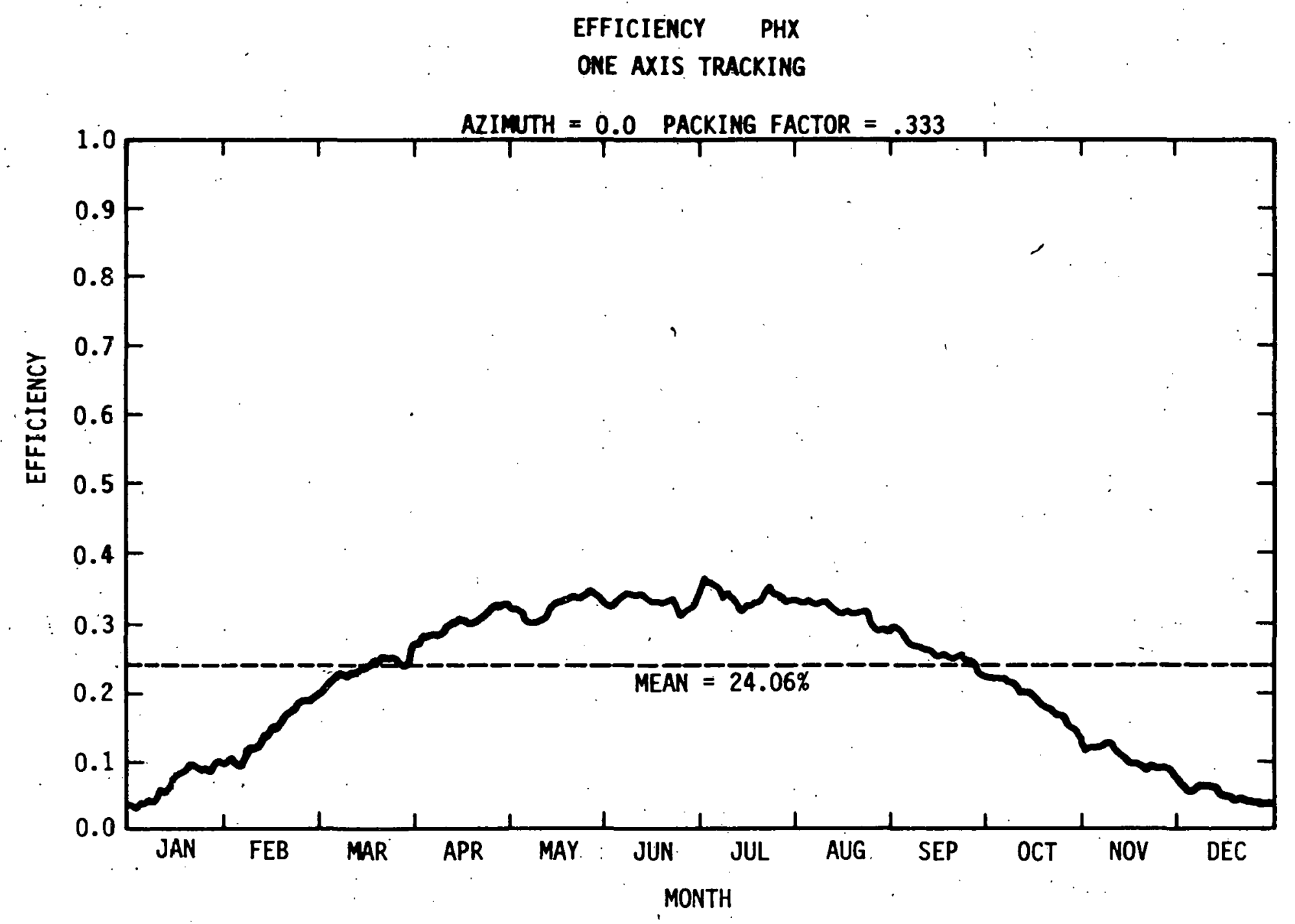

Figure 4. Calculated Collector Field Subsystem Efficiency: Continuously Cleaned Collectors 
Blank Page

88 
STEADY-STATE THERMAL LOSS TEST OF - THE COLLECTOR SUBSYSTEM AT THE COOLIDGE, ARIZONA, SOLAR IRRIGATION FACILITY, 15 APRIL 1980

\section{Purpose}

The purpose of this test was to study the steady-state thermal losses in the collector subsystem fluid loop at Coolidge, Arizona.

\section{Test Procedure}

On 15 April 1980, the collector subsystem was prepared by pointing the collectors toward the west in the morning and toward the east in the afternoon. The directions were chosen in order to allow the receiver to radiate to the sky while being shaded from the sun by the edge of its trough. The fluid loop was then put into operation by circulating the Caloria through the loop and the gas-fired heater. The heater was used to supply the heat required to bring the fluid loop up to temperature.

Thermal loss data were collected for six different combinations of flow rates and inlet Caloria temperatures. For each data point, the flow rate was fixed by fixing the speed of the collector subsystem pump. The inlet Caloria temperature was held at the desired level by the gas-fired heater. These conditions were maintained for approximately 2 . hours to achieve temperature stability throughout the subsystem prior to gathering the data at each test point.

The fluid loop schematic is shown in Figure 1 . As shown, the collector subsystem consists of eight individual collector loops. 


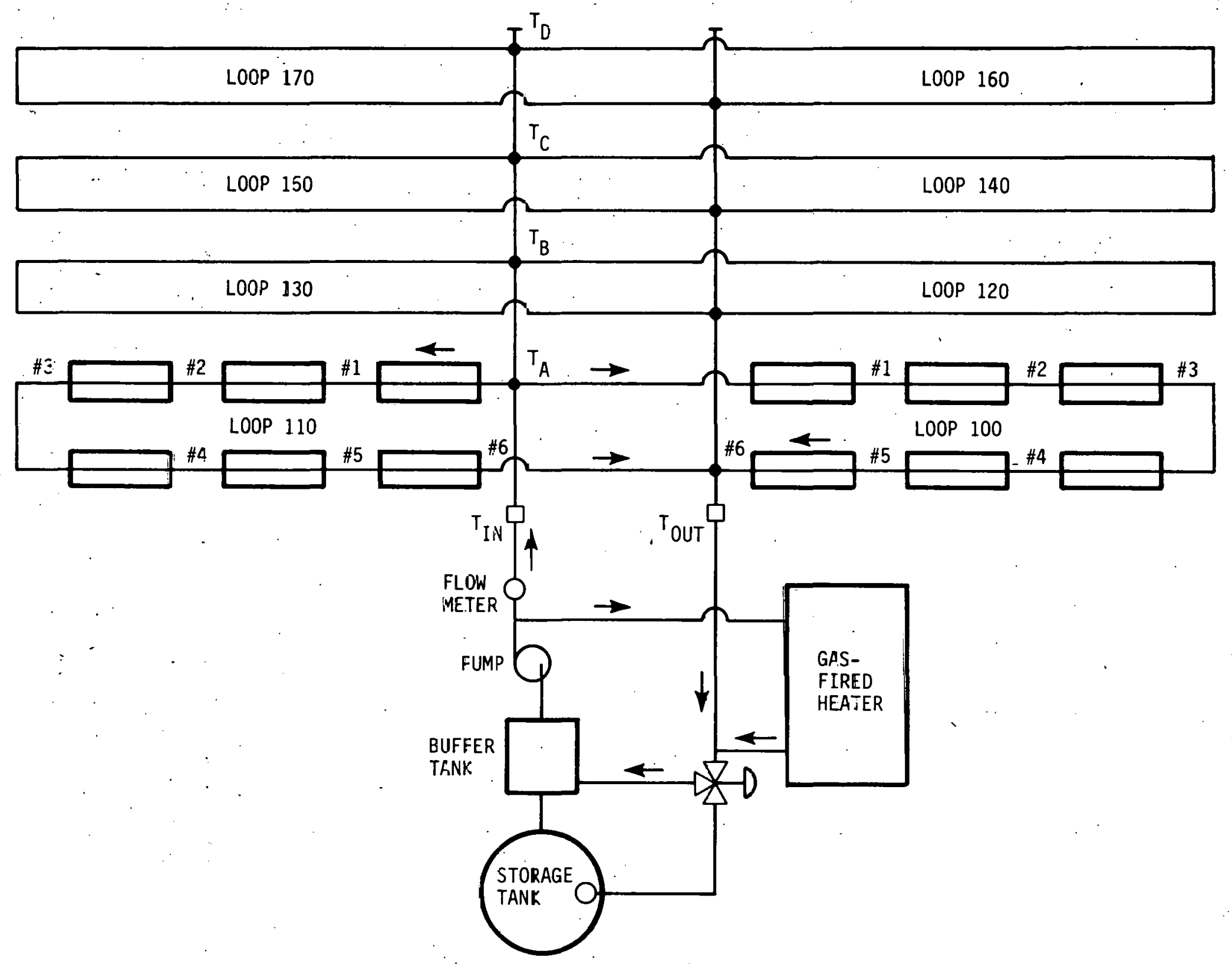

Figure 1. Fluid Loop Schematic 
Each loop consists of 48 collector modules plumbed in series." At the outlet of each string of eight collectors in each loop is a resistance temperature detector (RTD). They are numbered 1 through 6 in each loop as shown. At each test point, temperature readings were taken at each of these RTDs. In addition, the temperatures at the inlet to each loop, the inlet to the collector subsystem, and the outlet from the collector subsystem were determined by thermocouples, as shown.

\section{Test Results}

The test results are presented in Table 1 and Figure 2. Table 1 lists the data as collected at each test point. Figure 2 is a plot of the total thermal losses from the collector subsystem versus the midpoint receiver temperature above ambient. Midpoint receiver temperature is defined as the fluid temperature at the midpoint of the individual collector loops averaged over the field. In addition, included is a plot of the receiver losses alone as determined at the Collector Module Test Facility in Albuquerque, New Mexico, for the same collector modules. The thermal losses from the pipelines alone were then calculated by subtracting the receiver losses from the total losses. The results were plotted as the calculated pipe loss on Figure 2 :

\section{Discussion}

The calculated pipeline steady-state thermal losses in the collector subsystem will represent 88 of the energy collected on a good summer day. This rather high loss substantiates an increasingly strong feeling. at Sandia National Laboratories that "standard practice" in fluid transport system design needs to be reevaluated for solar applications.

The major known contributors to these heat losses are the numerous pipe anchors and valves in the collector subsystem pipelines. In addition, the insulation at the top of the flexhoses has slipped downward, exposing the top few inches to the atmosphere. 
Table 1

Coliected Temperature Data, ${ }^{\circ} \dot{F}$

\begin{tabular}{|c|c|c|c|c|c|c|c|c|c|c|c|c|c|c|c|c|c|c|c|c|c|c|c|c|}
\hline Loop No: & . & & & 00 & & & & & & $120^{\circ}$ & & & & & 14 & 40 & & & & & & 160 & & \\
\hline RTO No. & 1 & $\hat{E}$ & 3 & 4 & 5 & 6 & $i$ & 2 & 3 & 4 & 5 & 6 & 1 & 2 & 3 & 4 & 5. & 6 & 1 & 2 & 3 & 4 & 5 & 6 \\
\hline $\begin{array}{c}\text { Test Point } \\
\text { No. } \\
1\end{array}$ & 522 & 505 & 488 & 464 & 443 & 426 & 512 & 134 & 471 & 456 & 440 & 418 & 506 & 488 & 472 & 452 & 433 & 416 & 499 & 9. 486 & 6464 & 4448 & 8426 & 6409 \\
\hline 2 & 538 & $: 27$ & 512 & 501 & 4.87 & 488 & $5 \therefore 9$ & $51 ?$ & 500 & 492 & 481 & 468 & 523 & 511 & 501 & 4388 & 176 & 155 & 514 & 4508 & 8494 & 4486 & 6469 & 9459 \\
\hline 3 & 489 & 432 & $470^{\circ}$ & 762 & 451 & .443 & 431 & 472 & 458 & 452 & 444. & .434 & 476 & 465 & 459 & 449 & 4.39 & 130 & 469 & 9466 & 6454 & 4447 & 7434 & 4427 \\
\hline 4 & 471 & $\angle 58$ & 438 & 425 & 408 & 395 & 453 & 447 & 425 & 416 & 402 & 352 & 458 & 442 & 429 & 412 & 397 & 381 & 453 & 3443 & 3424 & 4409 & 9388 & 8373 \\
\hline 5 & 432 & 421 & 398 & 385 & 369 & 358 & 424 & 409 & 337 & 377 & 364 & 348 & 418 & 403 & 389 & $3 \bar{i} 3$ & 359 & 347 & 409 & 9401 & 1385 & 5.374 & 4357 & 7345 \\
\hline 6 & 442 & 439 & 424 & 417 & 408 & 401 & 434 & 427 & 415 & 411 & 403 & 393 & 430 & 423 & 416 & 407 & 399 & 392 & 425 & 5422 & 2413 & 3407 & 7397 & 7389 \\
\hline LOOE Mo. & & & & 10 & & & & & & 130 & & & & & & 50 & & & & & & 70: & & \\
\hline RTD No. & 1 & 2 & 3 & 4 & 5 & 6 & 1 & $\varepsilon$ & 3 & 4 & 5 & 6 & 1 & 2 & 3 & 4 & 5 & 6 & 1 & 2 & 3 & 4 & 5 & 6 \\
\hline $\begin{array}{l}\text { Test Point } \\
\text { No. }\end{array}$ & & & & & & & & & & & & & & & & & & & & & & & & \\
\hline 1 & 502 & 481 & 466 & 453 & 435 & 421 & 500 & 468 & 452 & 451 & 427 & 420 & 508 & 486 & 466 & 452 & $427^{\circ}$ & -416 & 506 & 6484 & 464 & 4438 & 8430 & 0410 \\
\hline 2 & 524 & 513 & 507 & 499 & 488 & 479 & 524 & $3 \hat{c} 0$ & 493 & 496 & 476 & 478 & 531 & 518 & 504 & 494 & 475 & 470 & 526 & 6511 & 1499 & 9480 & 0477 & 7469 \\
\hline 3 & 4.32 & 470 & 462 & 454 & 444 & 438 & 477 & 773 & 449 & 453 & 435 & 436 & 481 & 470 & 459 & 451 & 437 & 433 & 479 & 9467 & 457 & 7442 & 2440 & 0430 \\
\hline 4 & 465 & 441 & 425 & 410 & 394 & 383 & $: 58$ & 746 & 411 & 409 & 386 & 382 & 463 & 442 & 425 & $41 \bar{c}$ & 389 & 381 & 462 & 2443 & 3425 & 5398 & 8392 & 2375 \\
\hline 5 & 421 & 700 & 388 & 376 & 362 & .345 & -16 & 406 & 376 & 374 & 351 & 347 & 422 & 403 & 388 & $37 c$ & 352 & -345 & 419 & 9. 400 & 385 & 5362 & 2358 & 8345 \\
\hline 6 & 432 & 722 & 417 & 409 & 401 & 394 & $=30$ & 426 & 405 & 409 & 394 & 385 & 435 & 425 & 416 & 410 & 397 & 393 & 433 & 3423 & 3415 & 5401 & 1400 & 0391 \\
\hline
\end{tabular}


Table 1 (Continued)

Coliected Temperature Data, ${ }^{\circ} \mathrm{F}$

\begin{tabular}{|c|c|c|c|c|c|}
\hline $\begin{array}{c}\text { Test Point } \\
\text { No. }\end{array}$ & $\begin{array}{c}\text { Flow Rate } \\
\text { gpm }\end{array}$ & $\begin{array}{l}\text { TIn } \\
\left(O_{F}\right)\end{array}$ & $\begin{array}{l}\text { Tout } \\
\left(O_{F}\right)\end{array}$ & $\begin{array}{c}T_{A M I B} \\
\left(\dot{O}_{F}\right)\end{array}$ & $\begin{array}{l}\text { Hind } \\
\text { mph }\end{array}$ \\
\hline 1 & 30.0 & 540.0 & 416.0 & 85.4 & 0.0 \\
\hline$\ddot{2}$ & 60.5 & 545.4 & 472.0 & 88.4 & 1.3 \\
\hline 3 & 60.9 & 495.0 & 434.0 & 90.1 & 3.0 \\
\hline 4 & 29.0 & 488.4 & 380.0 & 87.4 & 3.6 \\
\hline 5 & 28.8 & 445.5 & 348.5 & 83.2 & 4.7 \\
\hline 6 & 60.3 & 448.0 & 393.0 & 72.4 & 0.0 \\
\hline
\end{tabular}

\begin{tabular}{|c|c|c|c|c|}
\hline $\begin{array}{c}\text { TEST POINT } \\
\text { No. }\end{array}$ & $\begin{array}{c}\mathrm{T}_{\mathrm{A}} \\
\left({ }^{\mathrm{O}}\right.\end{array}$ & $\begin{array}{c}\mathrm{T}_{\mathrm{B}} \\
\left(\mathrm{O}_{\mathrm{F}}\right)\end{array}$ & $\begin{array}{c}\mathrm{T}_{\mathrm{C}} \\
\left(\mathrm{O}_{F}\right)\end{array}$ & $\begin{array}{c}\mathrm{T}_{\mathrm{D}} \\
\left(\mathrm{O}_{\mathrm{F}}\right)\end{array}$ \\
\hline 1 & 538 & 537 & 535 & 533 \\
2 & 547 & 547 & 547 & 541 \\
3 & 494 & 494 & 494 & 491 \\
4 & 488 & 488 & 488 & 487 \\
6 & 445 & 443 & 443 & 440 \\
\hline
\end{tabular}




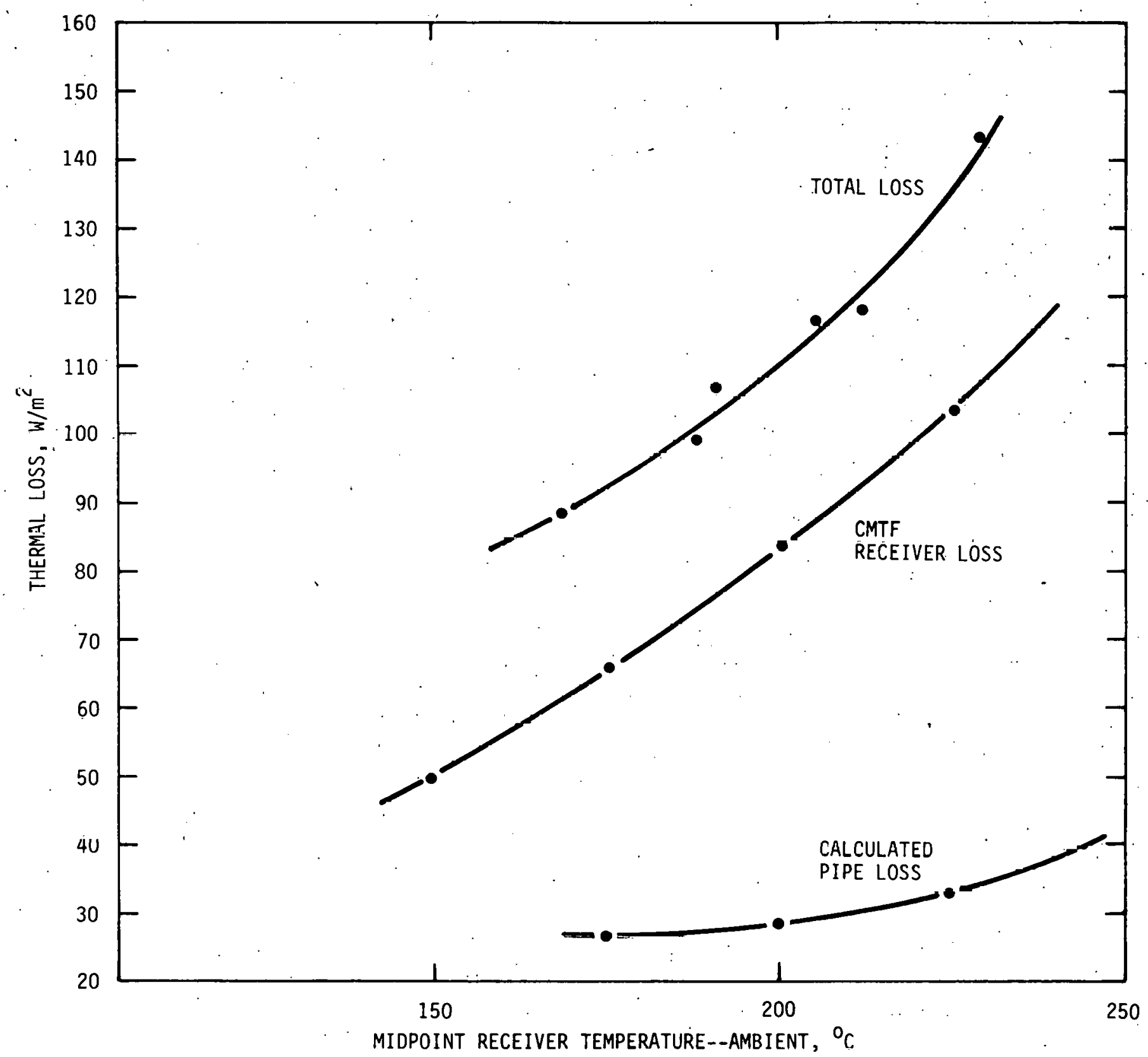

Figure 2. Total Thermal Losses vis. Midpoint Receiver Temperature above Ambient 
SUNDSTRAND ORGANIC RANKINE CYCLE SUBSYSTEM PERFORMANCE TESTS AT THE COOLIDGE, ARIZONA, SOLAR IRRIGATION FACILITY, 29, 30, AND 31 JANUARY`1980

\section{Subsystem Description}

The principal components of the Sundstrand Organic Rankine Cycle (ORC) Subsystem are the vaporizer assembly, power conversion module, and generator. The solar-generated thermal energy of the Caloria HT-43 from the storage tank is transferred to toluene in the vaporizer. The toluene is expanded through the turbine to drive the generator, producing electrical power. Figure 1 is a schematic of the entire 150-kW solar Irrigation Facility illustrating the subsystem components as they were installed.

The vaporizer assembly consists of three sections: the preheater, vaporizer, and superheater. The assembly is a fluid-to-fluid heat exchanger. A sketch of the equipment (Figure 2) identifies major items in the assembly.

Caloria that has been heated by the solar collectors is pumped through the superheater section, the vaporizer section, and the preheater sections and then pumped back through the solar collectors. At the same: time, toluene is pumped into the preheater through the vaporizer and superheater sections, transferring the heat from the Caloria to the toluene. This heat transfer vaporizes the toluene, which is then piped to the turbine on the power conversion module (PCM). A level sensor in the vaporizer section is provided to signal the level control valve on the PCM to open or close as needed to regulate the amount of toluene entering the vaporizer assembly. This mechanism ensures that the amount of toluene needed to meet the vapor requirements of the system is present in the vaporizer assembly. 


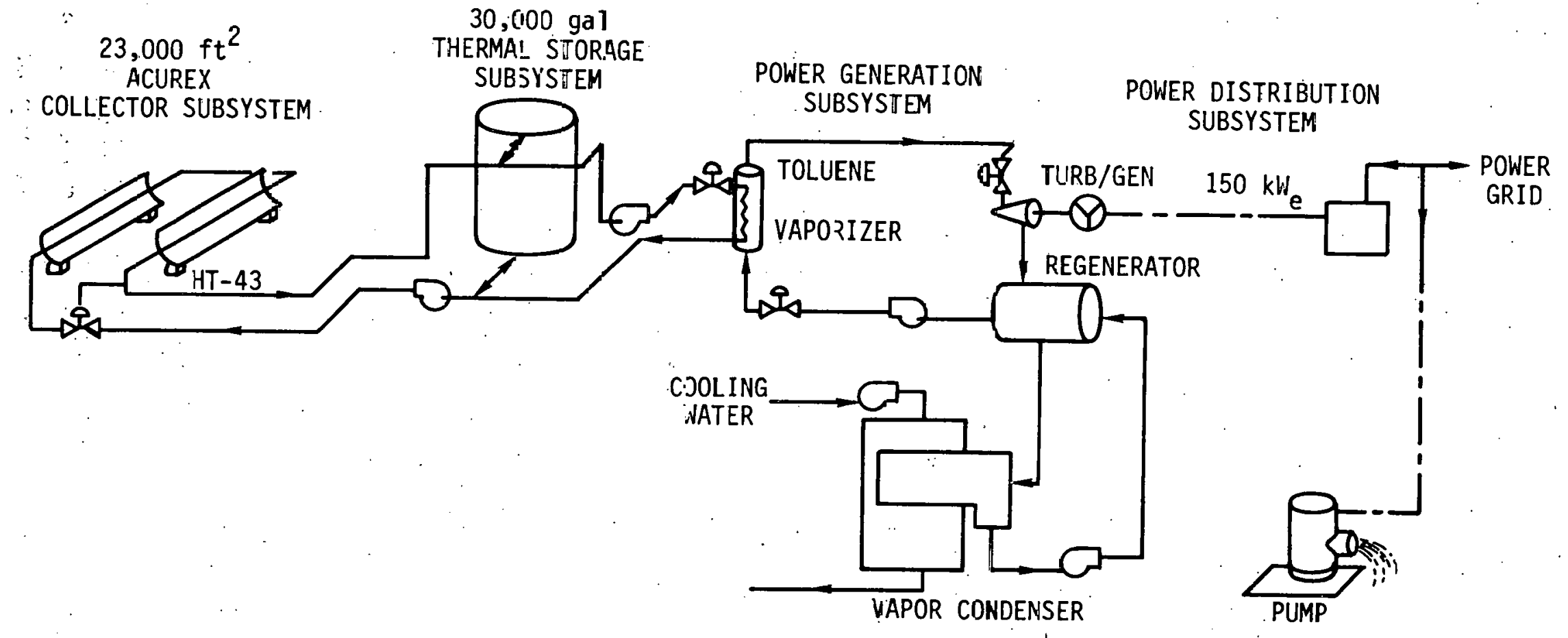

Figure 1. Schematic Diagram of the $150-\mathrm{kW}$ solar Irrigation Facility 


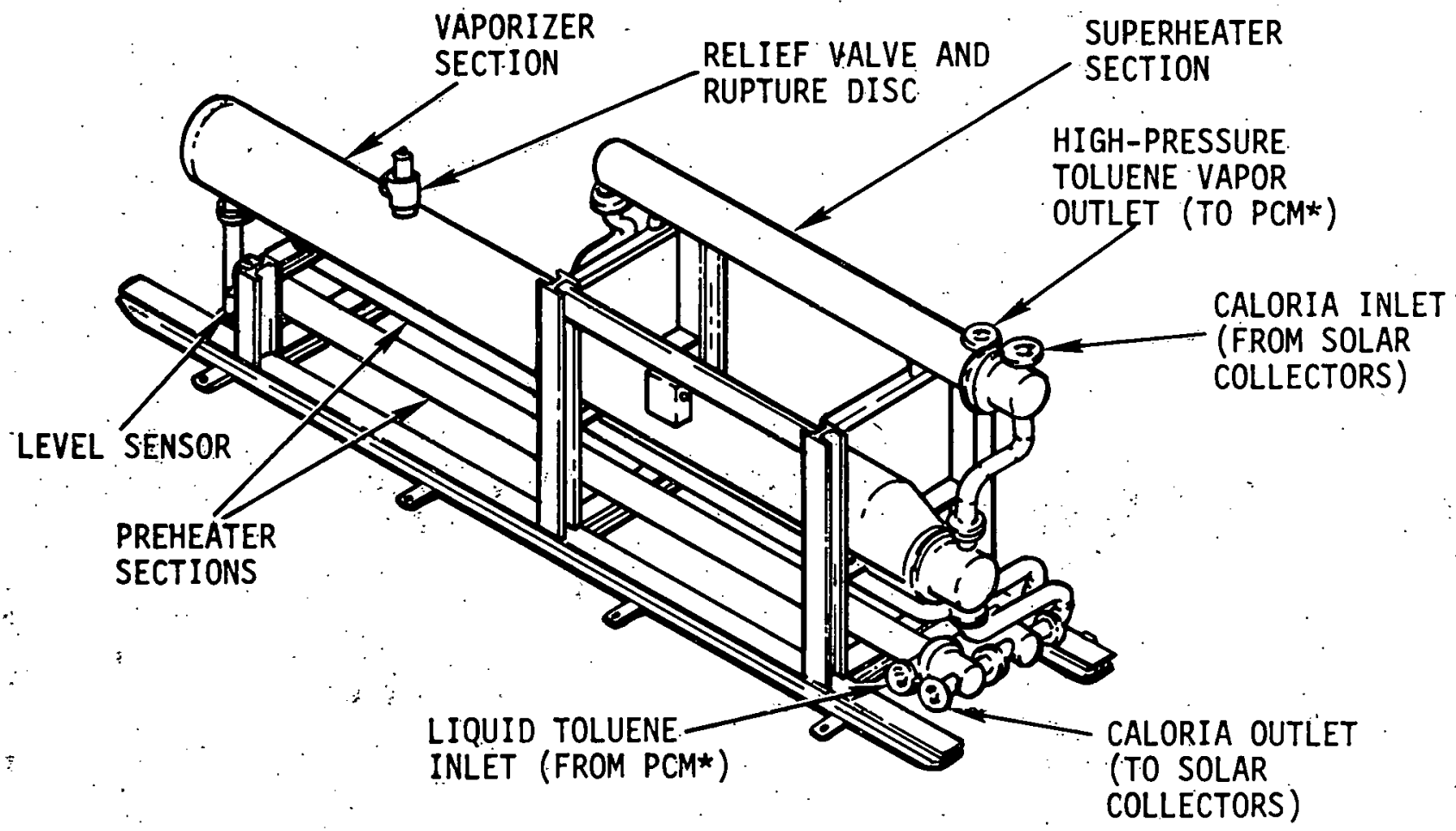

* power conversion module

Figure 2. Vaporizer Assembly 
The PCM components include the turbine/feed pump assembly; gearbox, regenerator assembly, noncondensable removal system, and liquid/ vapor toluene plumbing with related filters and pumps. These components are identified in. Figure 3. A description of the components follows .

1. Turbine/Feed Pump Assembly: The turbine/feed pump assembly is mounted on a welded framework at one end of the. PCM frame. The turbine exhaust outlet is connected to the regenerator by a bellows. The output shaft is connected to the gearbox by a flexible steel coupling. The two-stage main feed pump is mounted on the other end of the turbine shaft. The turbine bearings are lubricated with the working fluid, toluene. . The normal operating. speed of the turbine is $9,300 \mathrm{rpm}$.

2. Gearbox: The gearbox and turbine are mounted on opposite ends of a common support framework. This arrangement eases the shaft alignment and eliminates the relative motion of the shafts due to thermal expansion of the framework. The gearbox has an oil pump, sight glass, drain and fill plugs, and an oil filter. The oil is maintained at $93^{\circ} \mathrm{C}\left(199^{\circ} \mathrm{F}\right)$ by an external finned-tube oil cooler. The gearbox output speed is. maintained at $1,800 \mathrm{rpm}$.

3. Regenerator Assembly: The regenerator assembly houses the regenerator core: As high-temperature toluene vapor enters the houeing from the turbine, some of the vapor's heat is absorbed by the liquid toluene flowing through the regenerator core toward the vaporizer assembly (this preheats the liquid). As a result, less heat is needed at the vaporizer assembly to vaporize the toluene. The cooled vapor in the regenerator housing is then piped lo the vapor condenger assembly, where it is liquifled, and the condensate is returned to the hotwell. The system toluene level can be checked at three sight glasses on the side of the hotwell. 


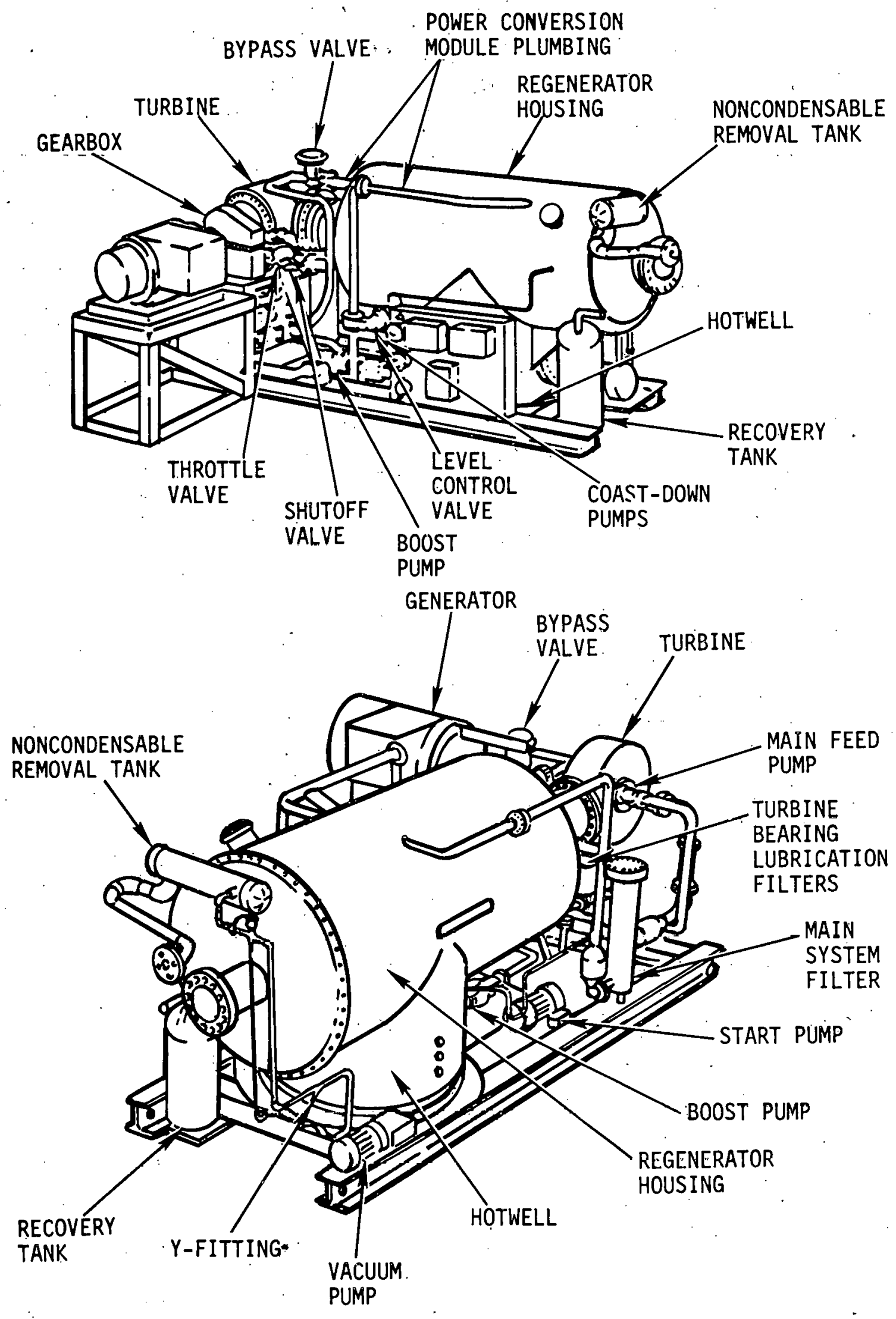

Figure 3. Power Conversion Module 
4. Noncondensable Removal System: The noncondensable removal system draws noncondensable gases (mostly nitrogen) from the vapor condenser core. Before system startup, a vacuum pump in the system lowers the pressure in the regenerator assembly to $20.7 \mathrm{MPa}(3.0 \mathrm{psia})$. (During steady-state operation, the pressure is maintained at $8.136 \mathrm{MPa}$ [1.18 psia].) As the system is running, the noncondensables are drawn from the core through a heat exchanger to condense the remaining toluene vapor. The condensed toluene flows from the heat exchanger to the vapor condenser before flowing to the hotwell. The remaining gases are drawn through a strainer before being pumped to the atmosphere by the vacuum pump. The system is activated as needed when a temperature differential between toluene vapor in the condenser and gas in the removal system indicates a buildup of nitrogen in the vapor condenser.

The primary piece of equipment in the heat rejection subsystem is the vapor condenser. It uses outside air and cascading waterflow to cool and condense toluene vapor passing through tube bundles.

After leaving the regenerator housing, the coluene vapur eirlers the vapor condenser tubes. Two fans force air over the tubes while a spray of water keeps the tubes wet. The resulting evaporation process cools and condenses the toluene vapor. The condensed liquid flows back to the hotwell on the PCM. The cooling water is recirculated, and additional water is automatically added to replace water lost in the evaporation process. The vapor condenser was manufduluied by Niagara Blower Company.

The main piece of equipment in the power distribution subsystem is the electrical generator. It is rated at $250 \mathrm{kVA}$ and 60 hertz and is a three-phase, synchronous, ac unit that operates at $1,800 \mathrm{rpm}$ with an output of 480 volts. The efficiency of the generator is 95.68 at 0.8 power factor. The generator was especially designed and manufactured for this solar system by Kato. 


\section{Purpose}

The purpose of this test series was to determine the performance characteristics of the sundstrand ORC Subsystem under design conditions and under a variety of off-design conditions.

\section{Test Procedure}

On 29, 30, and 31 January 1980, the Sundstrand ORC subsystem was operated utilizing heated Caloria HT-43 supplied from the thermal storage subsystem at a variety of flow rates and temperatures. Prior to each day's testing, the thermal storage tank was conditioned by the gas-fired heater.

\section{Test Results}

A listing of the data collected from the series of 16 tests is presented in Table 1. Figure 4 is a plot of gross cycle efficiency versus generator output. Gross cycle efficiency is defined as the generator electrical power output divided by the thermal power input to the vaporizer by the Caloria.

The design point parameters as provided by Sundstrand Corporation are presented in Table 2 so they may be compared to the test data.

Table 3 is a listing of all the parasitic electrical power consumed by the ORC subsystem over a range of generator output power. The parasitic power includes the power consumed by the Caloria pump at the vaporizer. These data are from an independent test series conducted by J. P. Abbin, Sandia. Laboratories, Albuquerque, New Mexico, on 3, 4, and 5 December 1979.

The test results indicate that the actual design point gross cycle efficiency is 19.7\%, which is very close to the manufacturer's projected value of 20.2\%. Subtracting the subsystem parasitic power, which averaged about $24 \mathrm{~kW}$ e $(22.7 \mathrm{Btu} / \mathrm{s})$, from the $200-\mathrm{kw}_{\mathrm{e}}$ (189.6-Btu/s) design point yields a design point net cycle efficiency of 17.38 . 
Table 1

Test Data

\begin{tabular}{|c|c|c|c|c|c|c|c|c|c|c|c|c|c|c|c|c|}
\hline Run Number & 1 & 2 & 3 & 4 & 5 & 6 & .7 & 8 & 9 & 10 & 11 & 12 & 13 & 14 & 15 & 16 \\
\hline Date & $1-29-80$ & $1-29-80$ & $1-29-80$ & $1-30-80$ & $1-30-80$ & $1-30-80$ & $1-30-80$ & $1-30-80$ & $1-30-30$ & $1-31-80$ & $1-31-80$ & $1-31-80$ & $1-31-80$ & $1-31-80$ & $1-31-80$ & $1-31-80$ \\
\hline Generator Output, $\mathrm{kW}_{\mathrm{e}}$ & 192.9 & 229.9 & 75.3 & 201.6 & 218.2 & 215.8 & 196.9 & 165.6 & 143.6 & 115.6 & 76.0 & 22.5 . & 168.0 & 206.3 & 189.4 & .106 .3 \\
\hline $\begin{array}{l}\text { Ambient Temperature, }{ }^{\circ} \mathrm{F} \\
\text { Toluene }\end{array}$ & 61.1 & 60.3 & 60.2 & 65.5 & 65.3 & 65.3 & 65.3 & 65 & 64.7 & 64.4 & 64.7 & 64.5 & 64.1 & 72.9 & 72.3 & 72.9 \\
\hline $\begin{aligned} \text { Hotwell, } & { }^{\circ} \mathrm{F} \\
\text { psia } & \end{aligned}$ & $\begin{array}{l}107.2 \\
0.634\end{array}$ & $\begin{array}{l}107.8 \\
0.786\end{array}$ & $\begin{array}{r}96.7 \\
0.295\end{array}$ & $\begin{array}{l}96.6 \\
0.53\end{array}$ & $\begin{array}{r}100.0 \\
0.53\end{array}$ & $\begin{array}{r}100.9 \\
0.53\end{array}$ & $\begin{array}{l}.99 .2 \\
0.53\end{array}$ & $\begin{array}{r}105.1 \\
0.77\end{array}$ & $\begin{array}{r}106.1 \\
0.53\end{array}$ & $\begin{array}{r}101.9 \\
0.53\end{array}$ & $\begin{array}{r}101.9 \\
0.28\end{array}$ & $\begin{array}{r}100.1 \\
0.28\end{array}$ & $\begin{array}{l}97.7 \\
0.77\end{array}$ & $\begin{array}{r}113.2 \\
0.33\end{array}$ & $\begin{array}{r}110.8 \\
0.72\end{array}$ & $\begin{array}{r}104.1 \\
0.38\end{array}$ \\
\hline $\begin{array}{l}\text { Regenerator Liquid } \\
\text { in, }{ }^{\circ} \mathrm{F}\end{array}$ & 110.3 & 110.9 & 99.3 & 99.1 & 102.2 & 103.7 & 102.0 & 106.2 & 105.7 & 100.4 & 97.9 & 96.6 & 99.1 & 114.8 & 113.3 & 105.3 \\
\hline Preheater in, ${ }^{\circ} \mathrm{F}$ & 229.1 & 230.1 & 237.2 & 239.5 & 242.4 & 242.3 & 244.5 & 253.4 & 251.3 & 245.3 & 242.1 & 243.1 & 242.0 & 245.7 & 261.1 & 262.1 \\
\hline Boiler, ${ }_{\text {psia }}^{\circ}$ & $\begin{array}{l}430 \\
160\end{array}$ & $\begin{array}{l}448 \\
189\end{array}$ & $\begin{array}{r}364 \\
80\end{array}$ & $\begin{array}{l}436 \\
168\end{array}$ & $\begin{array}{l}444 \\
180.2\end{array}$ & $\begin{array}{l}443 \\
179\end{array}$ & $\begin{array}{l}436 \\
166\end{array}$ & $\begin{array}{l}427 \\
151\end{array}$ & $\begin{array}{l}414 \\
134\end{array}$ & $\begin{array}{l}399 \\
116.2\end{array}$ & $\begin{array}{r}374 \\
89\end{array}$ & $\begin{array}{r}332 \\
55\end{array}$ & $\begin{array}{l}421 \\
151\end{array}$ & $\begin{array}{l}-- \\
184.7\end{array}$ & $\begin{array}{l}424 \\
151.7\end{array}$ & $\begin{array}{l}382 \\
99.7\end{array}$ \\
\hline Superheater out, ${ }^{\circ} \mathrm{F}$ & 491.0 & 480.1 & 472.2 & 499.0 & 493.0 & 490.8 & 492.7 & 495.8 & 488.5 & 477.2 & 462.8 & 434.3 & 485.5 & 507.9 & 523.6 & 511.3 \\
\hline Turbine in, ${ }^{\circ} \mathrm{F}$ & $\begin{array}{l}481.7 \\
139\end{array}$ & $\begin{array}{l}472.2 \\
165\end{array}$ & $\begin{array}{l}461.8 \\
67\end{array}$ & $\begin{array}{l}492.9 \\
147\end{array}$ & $\begin{array}{l}487.5 \\
158\end{array}$ & $\begin{array}{l}485.2 \\
157\end{array}$ & $\begin{array}{l}486.9 \\
146\end{array}$ & $\begin{array}{l}489.7 \\
132.5\end{array}$ & $\begin{array}{l}482.2 \\
116.7\end{array}$ & $\begin{array}{r}470.7 \\
99.7\end{array}$ & $\begin{array}{r}455.4 \\
77.0\end{array}$ & $\begin{array}{r}425.6 \\
47.1\end{array}$ & $\begin{array}{l}478.9 \\
132.7\end{array}$ & $\begin{array}{l}501.9 \\
160.0\end{array}$ & $\begin{array}{l}516.7 \\
131.2\end{array}$ & $\begin{array}{r}503.0 \\
84.7\end{array}$ \\
\hline Turbine out, ${ }^{\circ} \mathrm{F}$ & 313.6 & 307.7 & 311.4 & 334.9 & 332.6 & 330.9 & 334.9 & 347.2 & 341.6 & 337.3 & 331.8 & 324.9 & 334.6 & 340.3 & 360.0 & 354.9 \\
\hline Condenser Liquid out, ${ }_{{ }_{F}}$ & 110 & 113 & 83 & 96 & 99 & 99 & 96 & 109 & 96 & 91 & 85 & 77 & 98 & -- & 109 & 92 \\
\hline $\begin{array}{l}\text { Flow Rate, } 1 \mathrm{bm} / \mathrm{hr} \\
\text { Thermal Power Input, }\end{array}$ & 14,282 & 17,315 & 6,682 & 14,668 & 16,070 & 15,979 & 15,356 & 13,385 & 11,588 & 9,748 & 8,016 & 4,675 & 13,445 & 15,730 & 12,674 & 8049 \\
\hline $\mathrm{kW}$ & 1067 & 125.5 & 492.1 & 1.089 & 1.169 & 1.1 .58 & 11.1 .6 & 966 & 831.7 & 695.8 & 565.0 & 313.7 & 972.2 & 1169.1 & 950.0 & 595.6 \\
\hline Efficiency, $\frac{\circ}{\circ}$ & 18.07 & 18.32 & 15.30 & 18.51 & 18.67 & 18.63 & 17.64 & 17.15 & 17.26 & 16.61 & 13.45 & 7.17 & 17.28 & .17 .65 & 19.93 & 17.85 \\
\hline Caloria нт- 43 & & & & & & & & & & & & & & & & \\
\hline Superheater in, ${ }^{\circ} \mathrm{F}$ & 526.1 & 529.9 & 500.4 & 526.7 & 524.9 & 523.8 & 519.8 & 514.4 & 508.0 & 497.6 & 585.2 & 460.7 & 498.8 & 551.6 & 557.3 & 553.2 \\
\hline Preheater out, ${ }^{\circ} \mathrm{F}$ & 372.3 & 388.5 & 319.8 & 383.3 & 392.5 & 392.8 & 386.7 & 383.1 & 370.3 & 355.7 & 334.7 & 307.3 & 389.2 & 381.3 & 366.6 & 333.8 . \\
\hline Flow Rate, $1 \mathrm{bm} / \mathrm{hr}$ & 33,510 & 42,001 & 13,524 & 36,611 & 42,053 & 42,139 & 38,540 & 34,519 & 29,974 & 25,127 & 18,631 & 11,036 & 42,720 & 33,105 & 24,964 & 14,195 \\
\hline kw & 999 & 1159 & 460 & 1022 & 1086 & 1077 & 997. & 878 & 794 & 679.6 & 527.9 & 312.5 & 903.8 & 1106.2 & 931.2 & 601.0 \\
\hline Efficiency, : & 19.31 & 19.84 & 16.37 & 19.73 & 20.08 & 20.04 & 19.75 & 18.86 & 18.09 & 17.01 & 14.40 & 7.20 & 18.59 & 18.65 & 20.34 & 17.69 \\
\hline
\end{tabular}




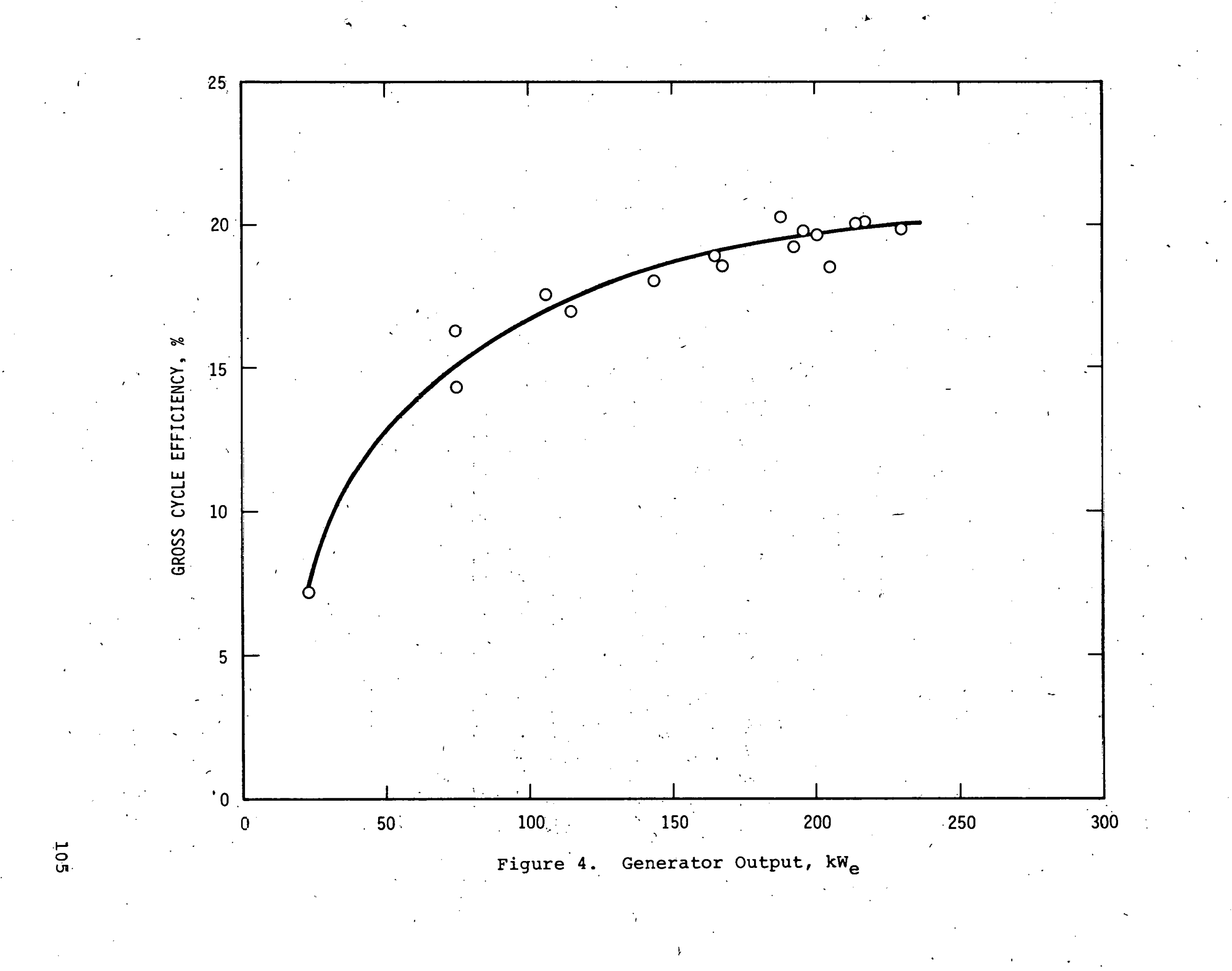


Table 2

Design Point Parameters

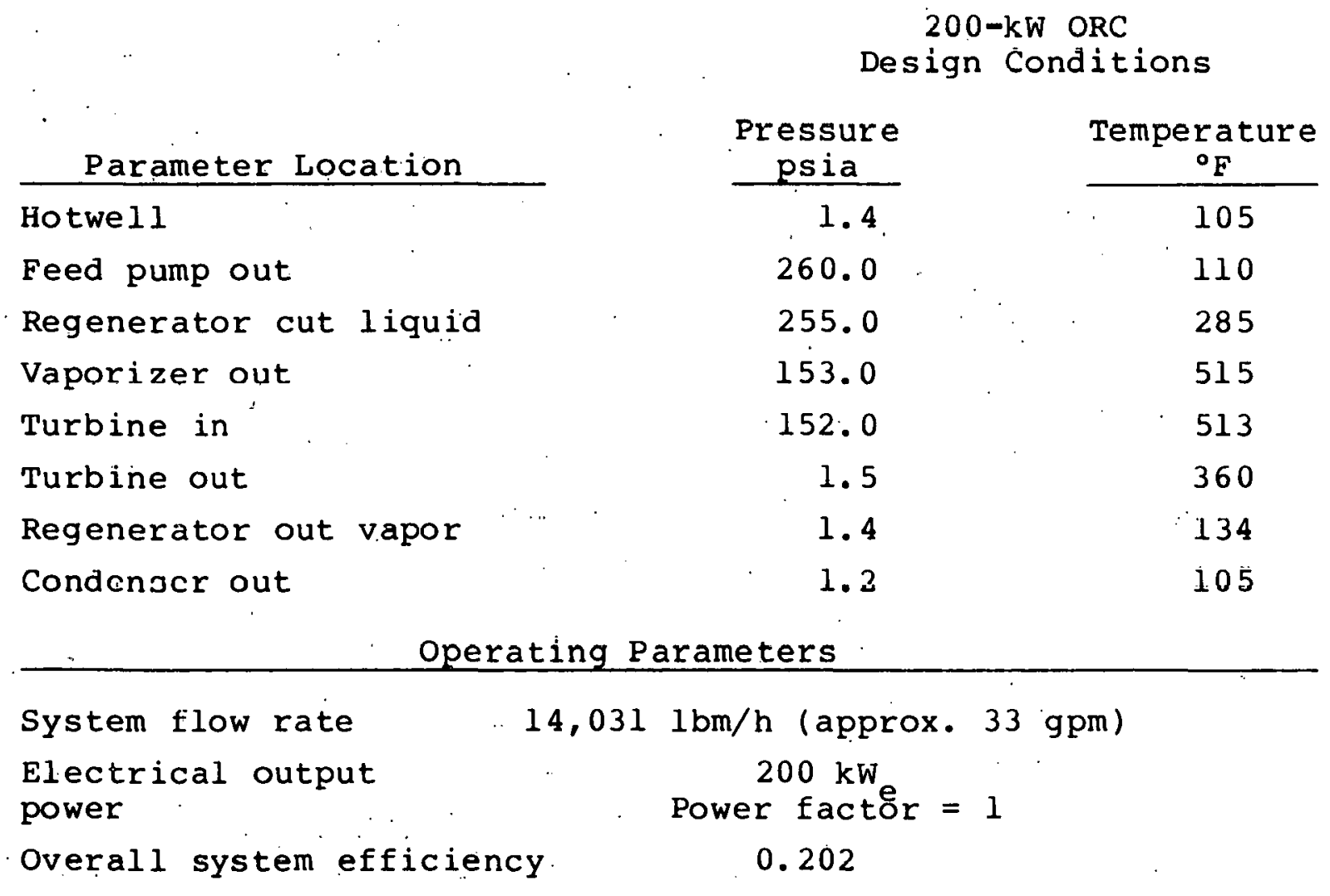


Table 3

Parasitic Power Consumption

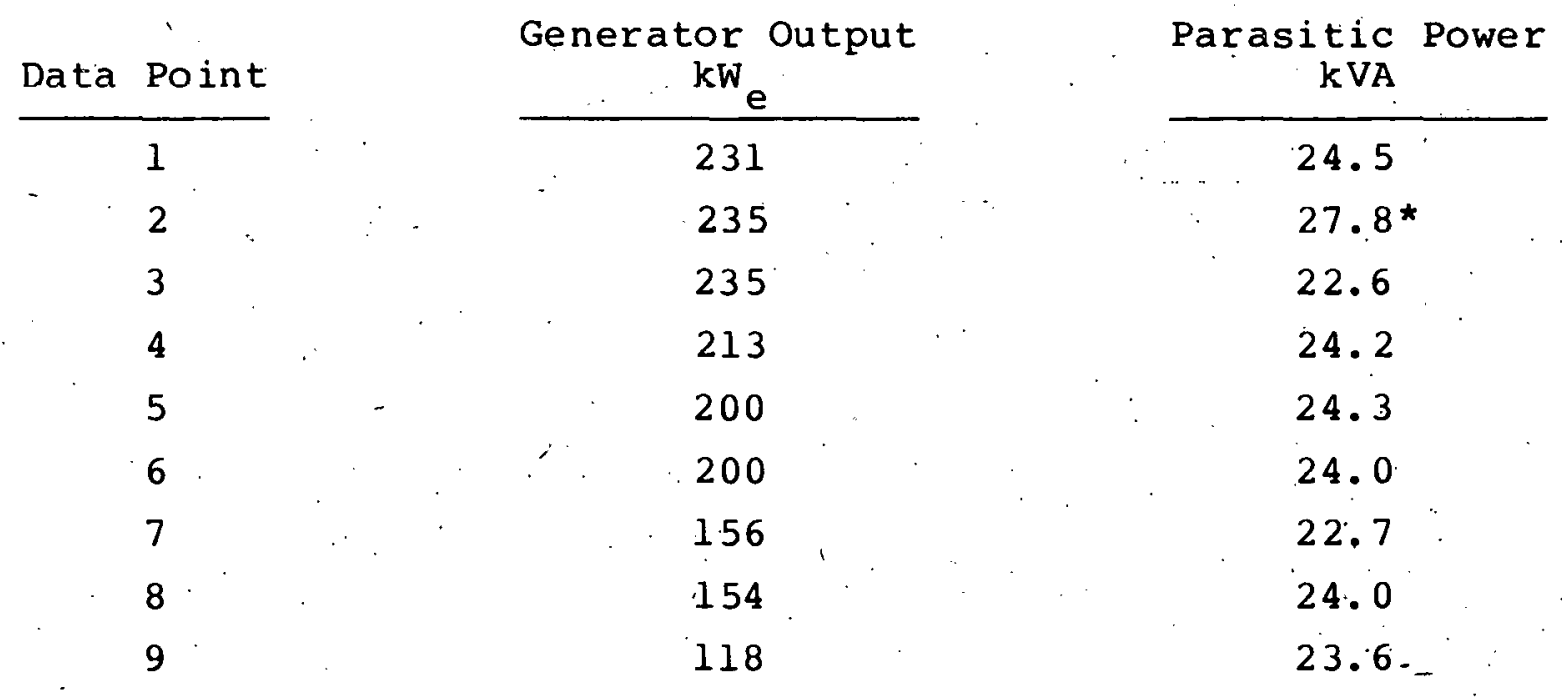

* Condenser vacuum pump running 
A breakdown of the parasitic power consumed, as determined by University of Arizona personnel, is as follows:

\begin{tabular}{|c|c|c|c|c|}
\hline Component & $\begin{array}{l}\text { Min. } \\
\mathrm{kW}_{\mathrm{e}}\end{array}$ & $\begin{array}{l}\text { Power } \\
\text { (Btu/s) }\end{array}$ & $\begin{array}{l}\operatorname{Max}_{\mathrm{e}} \\
\mathrm{kW}\end{array}$ & $\begin{array}{l}\text { Power } \\
(\text { Btu/s) }\end{array}$ \\
\hline Vaporizer Caloria pump & 4.0 & $(3.79)$ & 5.4 & $(5.12)$ \\
\hline Vapor condenser & 8.9 & $(8.436)$ & 8.9 & $(8.44)$ \\
\hline Power conversion module & $\underline{12.7 *}$ & $(12.04)$ & $\underline{15.1 *}$ & $(14.31)$ \\
\hline Total & 25.6 & $(24.26)$ & 29.4 & $(27.87)$ \\
\hline
\end{tabular}

The condenser vacuum pump consumes about $3.5 \mathrm{~kW}$ e $(3.32 \mathrm{Btu} / \mathrm{s})$ and runs infrequently: Subtracting $3.5 \mathrm{~kW}$ e $(3.32 \mathrm{Btu} / \mathrm{s})$ from the average total parasitic power listed above also results in $24 \mathrm{~kW}$ ( $22.8 \mathrm{Btu} / \mathrm{s})$.

\section{Discussion}

A key finding illustrated in Figure 4 is that the gruss cycle efficiency was nearly constant over a wide power range. The high efficiency over a wide power range is a valuable attribute in any power system, and these test results may indicate hie most energyefficient control strategy for systems with a variable luad. The subsystem power output for the tests was varied by manually regulating the hot Caloria flow from storage. Varying. the Caloria flow had the effect of moving the vaporizer boiler section boiling point in the same direction, i.e., decreasing the hot Caloria flow decreased the hoiling point while maintaining an essentially constant superheater outlet temperature and efficiency. This effect suggests a simple control scheme in which the turbine runs "wide open" at all times and only the heat input to the engine system is varied. As a consequence of this scheme, the turbine would not require expensive and complex variable admission or throttle devices; also, the turbine would see a minimum of thermal cycling due to variable inlet temperatures or 
variable mechanical loading on the turbine wheel blading, which. occur when a variable admission system is used.

Another item of interest from the tests was the difference between the engine efficiency calculated from thermal input based on the hot Caloria HT-43 measurements versus the toluene measurements. This difference could be explained by uncertainty about the properties of the Caloria or the toluene. Past experience at the Midtemperature Solar Systems Test Facility (MSSTF) and Willard seems to indicate that the toluene properties may be the culprit. This conclusion is based on the fact that at the MSSTF the engine efficiencies based on the T66 oil measurements have been consistently 5\% to $10 \%$ higher than those based on the toluene measurements, which are similar to the current test results at Coolidge. At Willard, however, the engine efficiencies based on the HT-43 measurements consistently agree with the Rl13 measurements within 5\%. Toluene has been used as a Rankine cycle working fluid for a relatively short time, and, thus, there has not been any particular emphasis on getting good thermodynamic properties. In light. of toluene's new role, research should be sponsored to accurately generate and verify its thermodynamic and heat transfer properties. 


\section{AN ESTIMATE OF THE PARASITIC ENERGY REQỤIREMENT OF THE COOLIDGE SOLAR IRRIGATION FACILITY}

The total energy requirement to operate the collector subsystem, power conversion subsystem, and control building is monitored on a daily basis. During May, June, and July, daily parasitic energy. consumption ranged from less than $200 \mathrm{kWh}$ e $\left(6.82 \times 10^{8} \mathrm{Btu}\right)$ up to $380 \mathrm{kWh}$ e $\left(13.0 \times 10^{8} \mathrm{Btu}\right)$, with the average use being $230 \mathrm{kWh}$ $\left(2.85 \times 10^{8} \mathrm{Btu}\right)$. On a representative, fully operational day, about $270 \mathrm{kWh}$ e $\left(9.21 \times 10^{8} \mathrm{Btu}\right)$ was used by the plant. Of this total, about half of the energy was used by the power conversion system. The collection system used an estimated $60 \mathrm{kWh}$ e $\left(2.05 \times 10^{8} \mathrm{Btu}\right)$, with the remainder required for the control building lights, air conditioner, and miscellaneous equipment.

The peak parasitic power requirement has been $41.3 \mathrm{~kW}$ (39.2 Btu/s). This demand is the sum of building, collector subsystem, and power conversion subsystem demands. Some of these demands have been quantified in short-term tests. The results of these tests follow.

\section{Collector Tracking Subsystem}

Power was measured as all tracking units were raised and lowered in unison at the control console in the control building. About $10.7 \mathrm{~kW}$ e $(10.14 \mathrm{Btu} / \mathrm{s})$ was used, of which about $0.05 \mathrm{~kW}$ e $(0.47 \mathrm{Btu} / \mathrm{s})$ was used by the console itself with tracking units inoperative. Thus, all tracker drive motors together required about $10.2 \mathrm{~kW}$ $(9.67 \mathrm{Btu} / \mathrm{s})$.

Average tracking system power requirements during normal tracking operation were computed by estimating the duty cycle for each motor 
run time was 2.68 of the operating time. The computed average power demand is 2.68 of $10.2 \mathrm{kw}$ e $(9.67 \mathrm{Btu} / \mathrm{s})$ or $0.3 \mathrm{kw}$ e $(0.28 \mathrm{Btu} / \mathrm{s})$ for the tracking system.

\section{Collector Field Pump}

The power required by the solar collector subsystem Caloria pump varied from $1.3 \mathrm{~kW}$ e $(1.23 \mathrm{Btu} / \mathrm{s})$ at a flow rate of $1.9 \mathrm{l} / \mathrm{s}$ $\left(30 \mathrm{gal} / \mathrm{min}_{1}\right)$ to $5.2 \mathrm{~kW}_{\mathrm{e}}(4.9 \mathrm{Btu} / \mathrm{s})$ at $5.2 \mathrm{l} / \mathrm{s}(82 \mathrm{gal} / \mathrm{min})$ for Caloria at about $205^{\circ} \mathrm{C}\left(400^{\circ} \mathrm{F}\right)$.

\section{Vaporizer Caloria Pump}

Vaporizer Caloria pump power demand was measured with different Caloria temperatures and generator outputs. 'l'he power requirement ranged from about $4.0 \mathrm{~kW}_{\mathrm{e}}(3.79 \mathrm{Btu} / \mathrm{s})$ to $5.4 \mathrm{~kW}$ e $(5.12 \mathrm{Btu} / \mathrm{s})$.

\section{Condenser cooling Tower}

The two-wattmeter method was used to measure power used by the cooling tower water pump and two fans. Each fan requires about $3.8 \mathrm{~kW} \cdot(3.60 \mathrm{Btu} / \mathrm{s})$; the water pump uses about $1.3 \mathrm{~kW}$ ( $(1.23 \mathrm{Btu} / \mathrm{s})$. Together, these three units use about $9 \mathrm{~kW}$ ( $8.5 \mathrm{Btu} / \mathrm{s})$ during operation.

\section{Power Conversion Module}

Power was measured at the load side of the 125-ampere PCM console circuit breaker in panel HA. Measurements ranging from $12.7 \mathrm{~kW}_{\mathrm{e}}$ $(12.04 \mathrm{Btu} / \mathrm{s})$ to $15.1 \mathrm{~kW}_{\mathrm{e}}(14.31 \mathrm{Btu} / \mathrm{s})$ were obtained with the turbine operating and the generator supplying power to the utility electrical grid system.

\section{Summary}

Daily parasitic electrical energy usage by the solar power plant averaged about $230 \mathrm{kWh}$ e $\left(7.85 \times 10^{8} \mathrm{Btu}\right)$ during May, June, and July. A more representative value for a fully operational day is estimated 
to be $270 \mathrm{kWh}$ e $(9.21 \times 108 \mathrm{Btu})$. Approximately half the energy is used by the power conversion module, $20 \%$ by the collector subsystem, and 308 by the control building including air conditioner. 
Blarte Page

114 
STATIC TEST OF THERMOCLINE STORAGE TANK AT COOLIDGE, ARIZONA, 14 THROUGH 1.6 NOVEMBER 1979

\section{$\underline{\text { Purpose }}$}

The purpose of this test was to study the rate of growth of the thermocline in the thermal storage tank at coolidge under static conditions.

\section{Test Procedure}

The tank was prepared by first heating it uniformly to $287^{\circ} \mathrm{C}$ $\left(550^{\circ} \mathrm{F}\right)$ from the top down to the lower diffuser, using the gas-fired heater. To establish a thermocline, the Organic Rankine Cycle (ORC) subsystem was operated with a $200-\mathrm{kW}_{\mathrm{e}}(189.6-\mathrm{Btu} / \mathrm{s})$ output. The temperature of the Caloria returned to the bottom of the storage tank by the ORC subsystem was $160^{\circ} \mathrm{C}\left(320^{\circ} \mathrm{F}\right)$. At the end of this process, the temperature profile in the tank was as shown by the solid line in Figure 1 .

The initial thickness of the thermocline was probably caused by turbulence at the lower diffuser both during the heating process and during. the initial injection of colder Caloria from the ORC subsystem. The upper diffuser was, closed off, and the lower diffuser pipeline was left open to allow the tank to act as an expansion tank for the remainder of the system. The tank was held in this condition for the duration of the test.

The test began at 8:15 p.m., Wednesday, 1:4 Noyember, and ended 1:43 p.m., Friday, 16 November. The temperature profile of the Caloria in the tank was determined by monitoring type $K$ thermocouples on the outside of the skin of the tank. These had been welded to the tank prior to the installation of the insulation. 


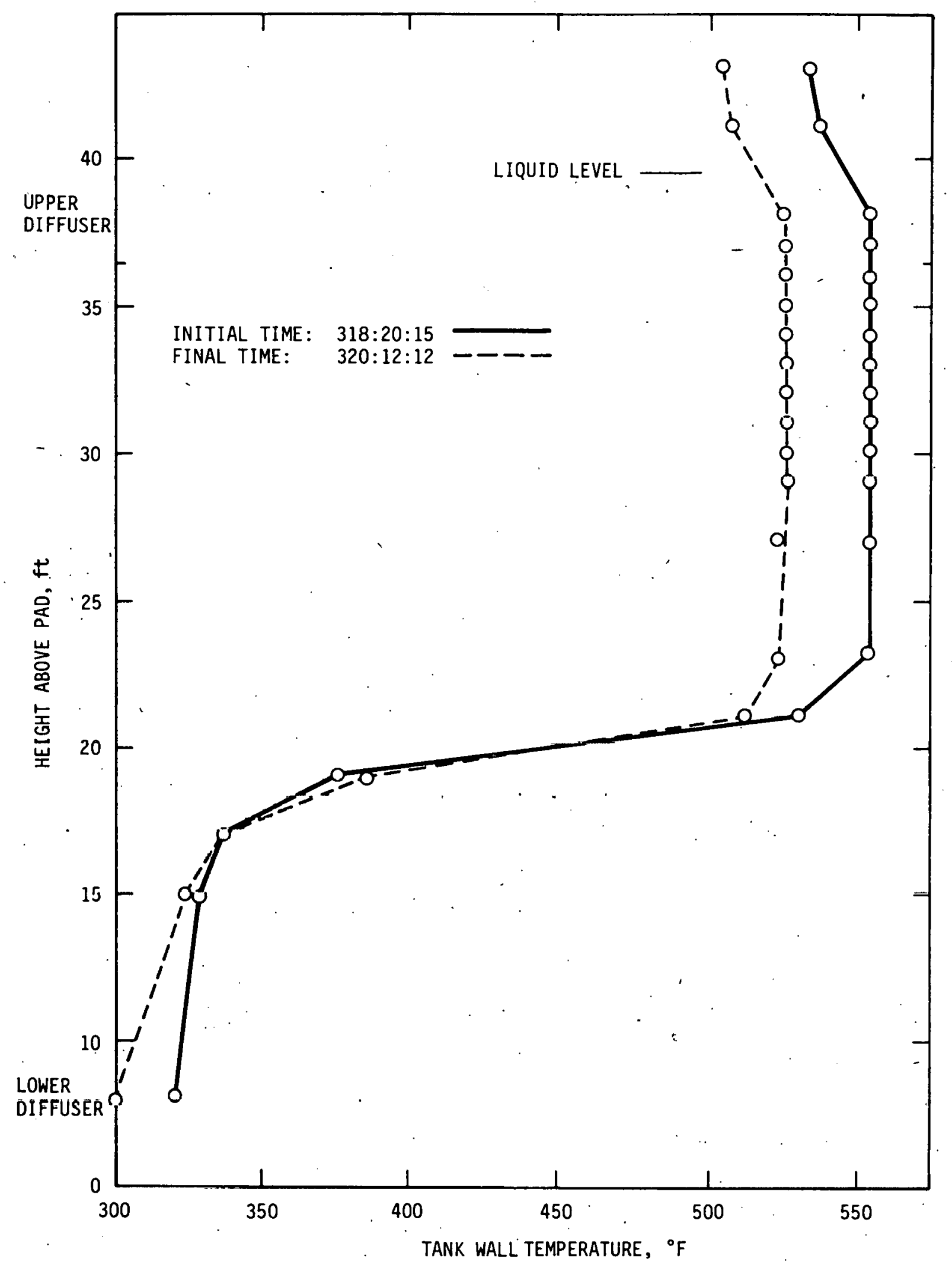

Figure 1: Thermal storage Tank Temperature Profile 


\section{Test Results}

The test results are presented in Figures I through 4. Figure I presents the temperature profile of the skin of the tank at the beginning and end of the test. Figure 2 is a plot of the upper bulk oil temperature versus time during the test. Figure 3 shows the temperature at several points on the dome of the tank and on the manhole cover on the side of the tank. Figure 4 is a plot of the ambient air temperature and wind speed during the test.

\section{Discussion}

The vertical resistance temperature detector (RTD) probe in the center of the tank was used only to verify that no horizontal temperature gradient existed in the upper portion of the tank. The other RTDs were not yet properly set up. It was then assumed that the skin temperature of the tank would be equal to the Caloria temperature all the way down the tank.

The thermocline proved to be stable and did not grow in size throughout the test. However, the upper bulk Caloria temperature decreased at the rate of $0.381^{\circ} \mathrm{C}\left(0.686^{\circ} \mathrm{F}\right)$ per hour. The purpose of presenting the extra thermocouple readings on the thermal storage tank, the wind velocity, and the ambient air temperature in the test results is to permit a. study of the thermal loss mechanisms that are present. 


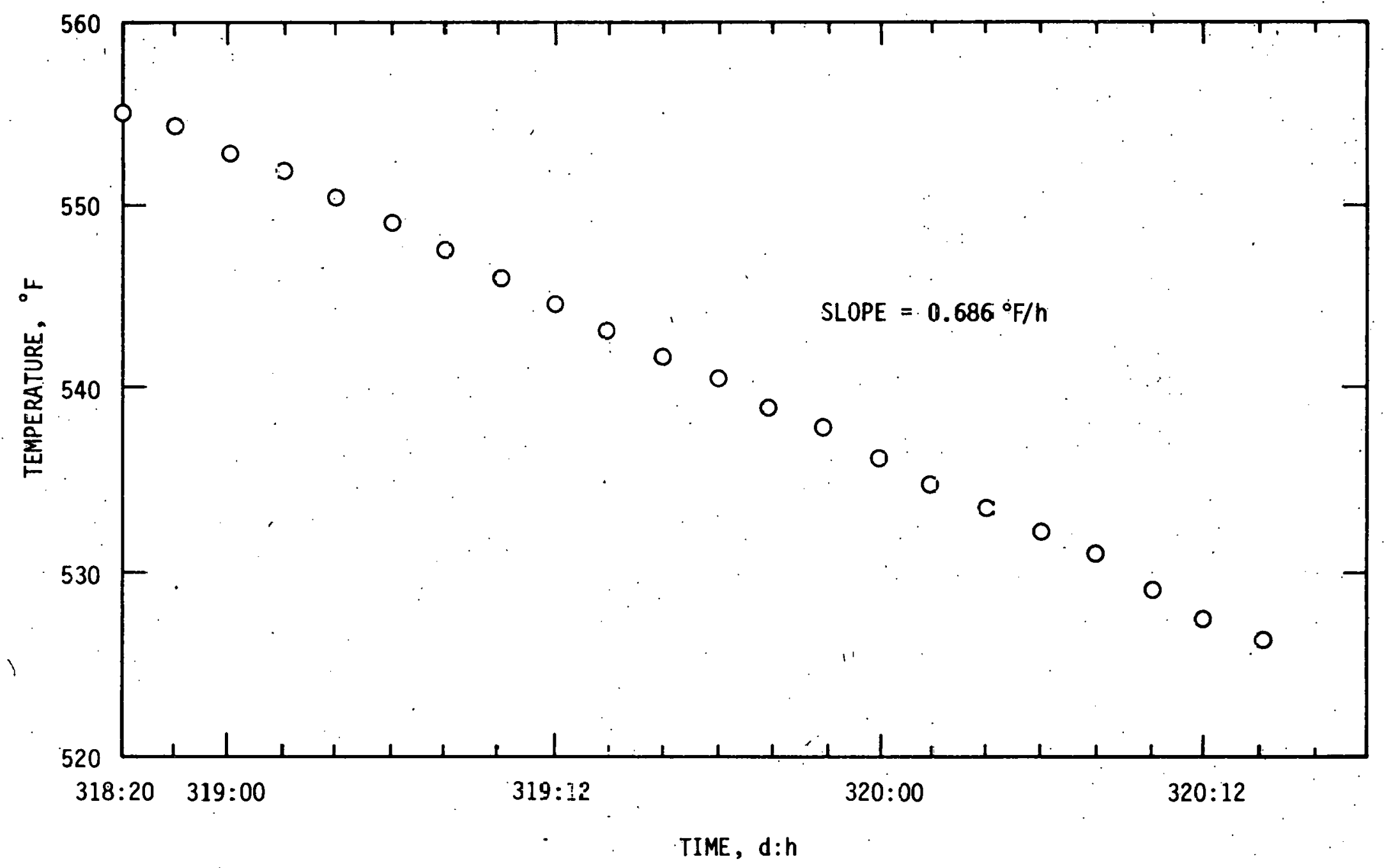

Figure 2. Upper Bulk Caloria Temperature vs. Time 


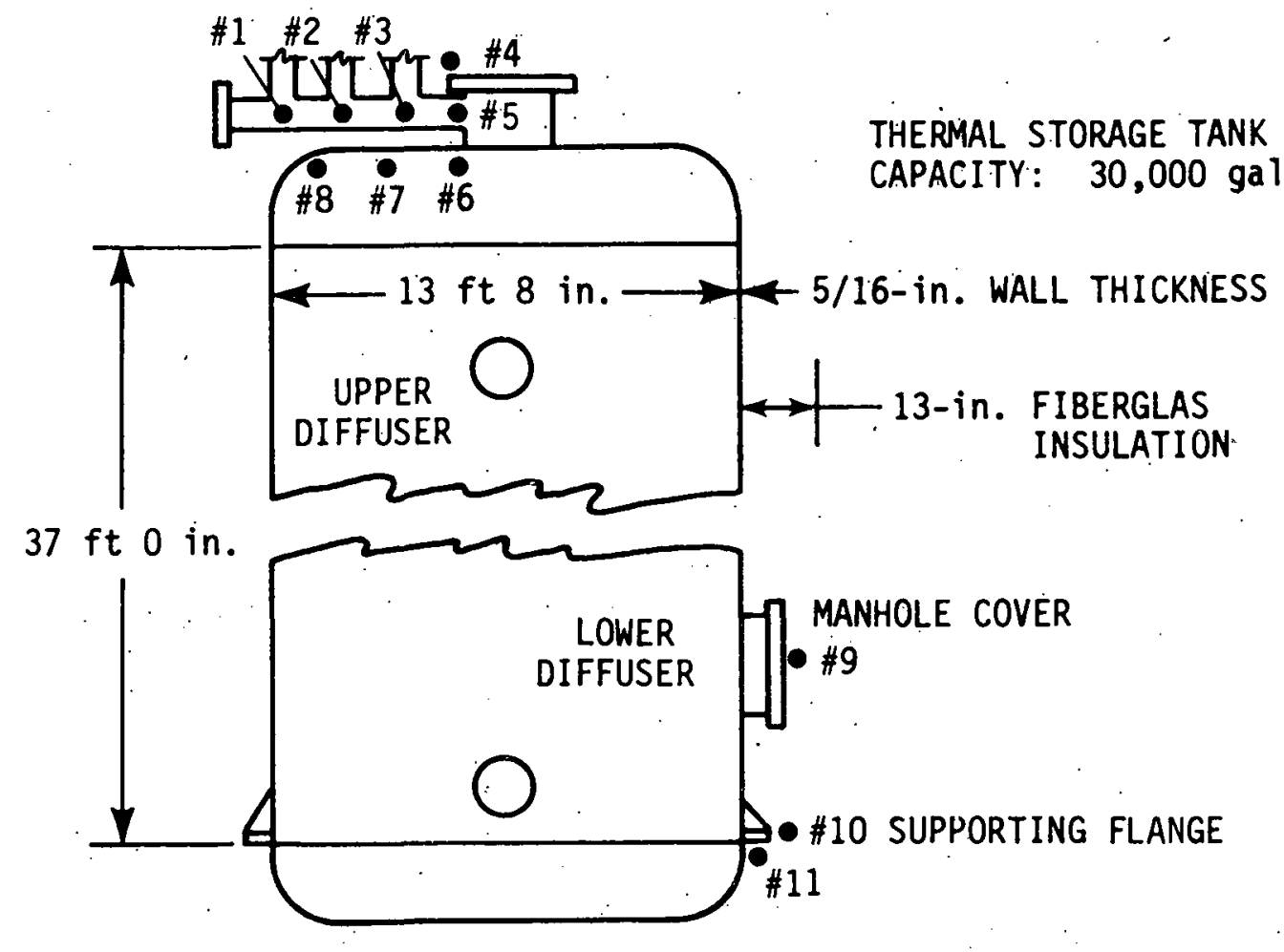

TIME THERMOCOUPLE TEMPERATURE, ${ }^{\circ} \mathrm{F}$

\begin{tabular}{|c|c|c|c|c|c|c|c|c|c|c|c|}
\hline DAY:HR:MIN & $\# 1$ & $\# 2$ & $\because 33$ & $\# 4$ & $\# 5$ & 洪6 & $\# 7$ & $\# 8$ & $\# 9$ & $\# 10$ & $\# 11$ \\
\hline $318: 20: 15$ & 74.7 & $85.3 \cdots$ & 111.5 & 174.7 & 307.2 & 465.6 & 528.1 & 536.5 & $\therefore-$ & 174.7 & 144.3 \\
\hline $19: 00: 00$ & 73.8 & 85.6 & 112.8 & 175.3 & 309.6 & 464.5 & 524.8 & 534.0 & $\because--$ & 171.8 & 140.0 \\
\hline $19: 04: 00$ & 64.9 & 77.0 & 104.5 & 176.9 & 307.8 & 465.3 & 522.0. & 531.3 & --- & 169.9 & 134.9 \\
\hline 319:08:00 & 74.5 & 74.3 & 99.1 & 172.9 & 308.5 & 466.7 & 519.6 & 528.6 & --- & 166.3 & 130.8 \\
\hline 2:00 & 114.3 & 108.1 & 129.0 & 183.7 & 311.3 & 463.3 & 517.6 & 525.9 & --- & 171.1 & 43.2 \\
\hline $319: 16: 00$ & 101.7 & 108.7 . & 130.6 & 184.8 & 315.9 & 460.0 & & & $\cdots$ & 177.6 & 152.9 \\
\hline $319: 20: 00$ & 81.3 & 94.6 & 122.9 & 177.1 & 311.4 & 458.6 & 511.7 & 519.1 & --- & 175.5 & 146.5 \\
\hline $320: 00: 00$ & 76.8 & 90.5 & 119.8 & 176.5 & 314.6 & 458.1 & 509.0 & 516.5 & -- & 171.1 & 140.4 \\
\hline $320: 04: 00$ & 72.3 & 86.2 & 116.2 & 177.1 & 317.5 & 456.1 & 506.3 & 513.5 & -- & 168.6 & 136.9 \\
\hline 320.08 .00 & 72.9 & 73.6 & 98.8 & 174.7 & 321.4 & 455.5 & 503.6 & 510.8 & 203.2 & 163.8 & 129.2 \\
\hline $320: 12: 12$ & 119.5 . & 115.2 & 138.2 & 198.1 & 320.9 & 453.9 & 500.9 & 508.6 & -- & 167.5 & 140.9 \\
\hline
\end{tabular}

Figure 3." Extra Thermocouple Readings on Thermal Storage Tank 


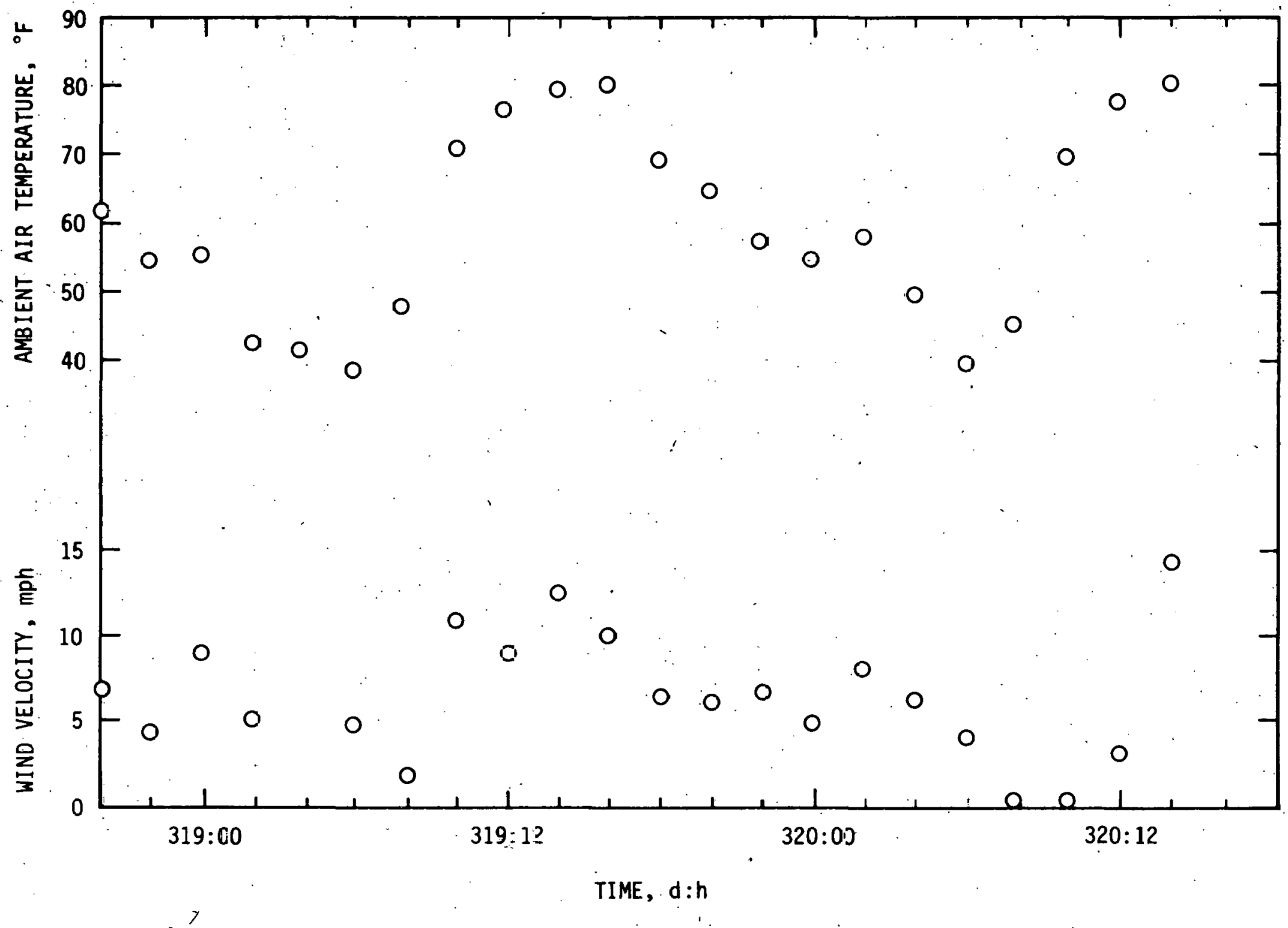

Figure 4. Ambient Air Temperature and wind Velocity vs. Time 
COOLIDGE, ARIZONA, THERMAL STORAGE SÜBSYSTEM

THERMOCLINE GROWTH TEST,

17 THROUGH 21: APRIL 1980

\section{$\underline{\text { Purpose }}$}

The purpose of this test was to illustrate the growth characteristics of a thermociline established and maintained in the storage tank at Coolidge, Arizona, over a 5-day period with the system in daily operation.

Significant leakage through the three-way bypass valve obscured the test results. This test will be repeated after a new three-way valve is installed. The test report is presented here for general information.

\section{Test Procedure}

For the purpose of this test, the thermocline is defined as that. layer of fluid in the storage tank that has a temperature of $260^{\circ} \mathrm{C}$ $\left(500^{\circ} \mathrm{F}\right)$ on top and $232^{\circ} \mathrm{C}\left(450^{\circ} \mathrm{F}\right)$ on the bottom. The thickness of the thermocline is the distance separating the $260^{\circ} \mathrm{C}\left(500^{\circ} \mathrm{F}\right)$ and $232^{\circ} \mathrm{C}$ $\left(450^{\circ} \mathrm{F}\right)$ Caloria.

In the course of normal operation at coolidge, any thermocline that may have formed in the storage tank during the day is purged from the top of the tank at the end of the day and utilized by the Organic Rankine Cycle (ORC) subsystem. This procedure is made possible by the flexibility of the ORC subsystem, i.e., the ORC will operate when the temperature of the Caloria supplied to it ranges between $287^{\circ} \mathrm{C}\left(5.50^{\circ} \mathrm{F}\right)$ and $215^{\circ} \mathrm{C}\left(420^{\circ} \mathrm{F}\right)$. 
In order to allow a thermocline" to form and grow for the 5-day test period, the following restraints were imposed on the system.

1. The ORC subsystem was stopped when the storage tank could no longer supply it with Caloria at $260^{\circ} \mathrm{C}\left(500^{\circ} \mathrm{F}\right)$ or above and

2. Flow from the bottom of the storage tank to the collector subsystem was allowed only when the temperature of the Caloria being supplied was at $232^{\circ} \mathrm{C}\left(450^{\circ} \mathrm{F}\right)$ or less.

In addition, the tank was initially conditioned for the investigation by cooling its storage volume below $221^{\circ} \mathrm{C}\left(430^{\circ} \mathrm{F}\right)$.

On 17 April 1980 at 10:20 a.m., the system was put into operation. The above initial condition and operating restraints lad been imposed on the system. This insured that once the system was put into operation a thermocline would exist and be maintained in the storage tank.

During the course of this test, the thermocline was first moved down and then up daily. This was accomplished by operating the collectors alone until midafternoon. Then the ORC subsystem was started and operated simultaneously with the collectors until the thermocline reached the top of the storage tank. The system was then stopped for the night. The procedure was followed during the first three days ot the test. The last two days were mostly cloudy, and insufficient energy was collected for turbine operation.

The temperature profile of the Caloria in the storage tank was monitored daily at collector subsystem startup, at ORC startup, and at system shutdown. The thermocouples welded to the outside of the storage tank were used to obtain the temperature readings.

Two diffusers exist in the top of the storage tank, as shown in Figure 1. The lower of the two was valved off during this entire test series. 


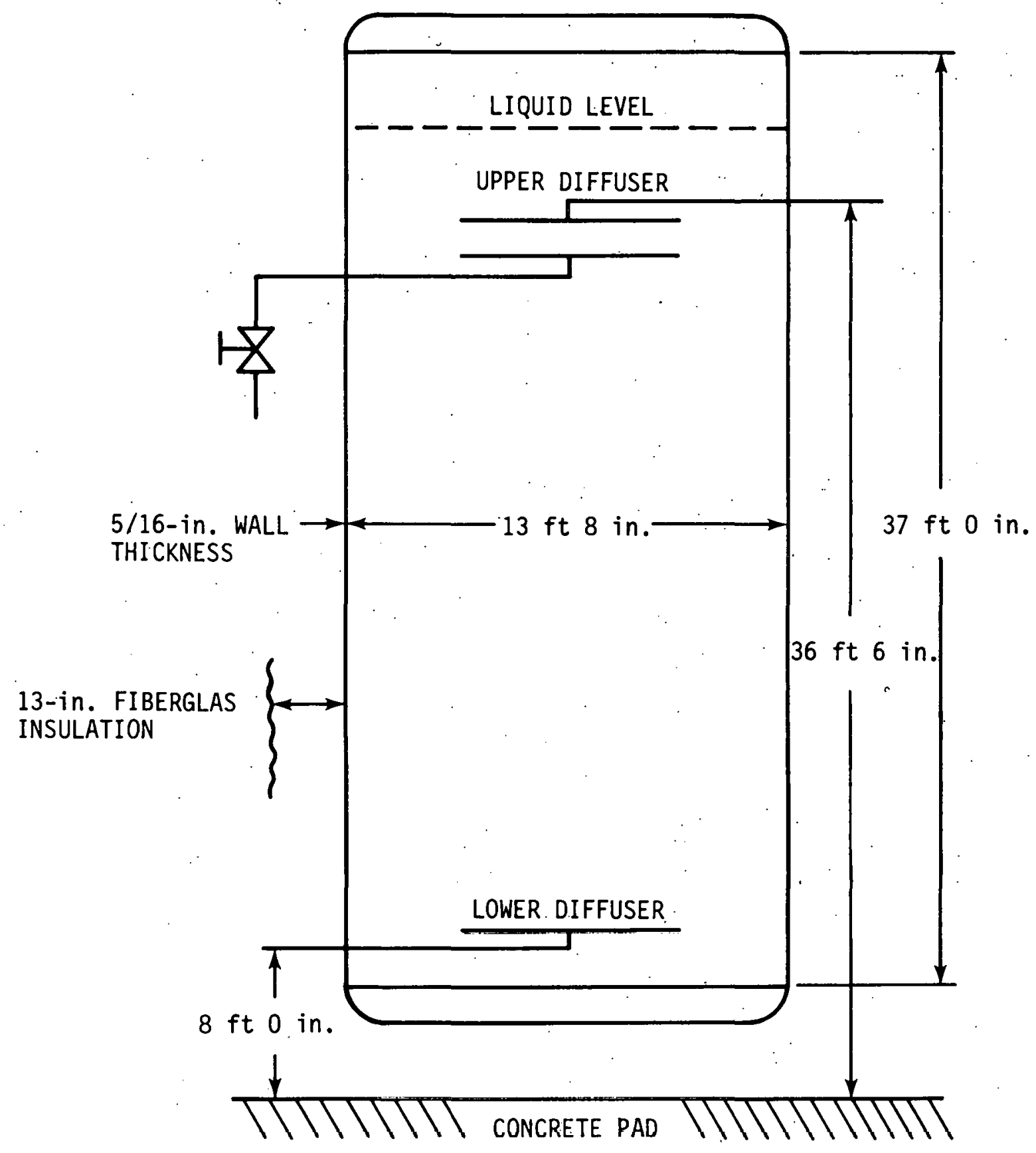

Figure 1. Tank Configuration 


\section{Test Results}

The test results are presented graphically in Figures 2 through 7. Figure 2 shows how the thickness of the thermocline changed with time. Figures 3 through 7 show the temperature profiles of the storage tank as they were measured each day.

Figure 2 shows the thermocline thickness changing in discrete steps in a regular pattern during the first two days, followed by only minor changes on the third day. "These steps are identified as "a,". "b," and "c." step "a" occurred during the first portion of the day, when the collectors alone were in operation. Step "b" occurred during the time when the collectors and ORC subsystem were in simultaneous operation. At the end of step "b," the thermocline was at the top of the storage tank. During step "c," the thermocline remained static at the top of the storage volume overnight.

Little change was observed in the thermocline thickness while it remained static. This result agrees with a test reported earlier on the growth rate of the thermocline under static conditions.

The extreme growth of the thermocline on the fourth day occurred during midday, when the sky was "mostly cloudy." During this time, the flow through the collector field was held in a circulating mode by the bypass valve for 5 hours. The purpose of the bypass valve is to allow the Caloria to circulate through the collector field during its warmup period at the beginning of the day or during a period of insufficient insolation. Whenever the Caloria temperature at the collector field outlet exceeds $279.4^{\circ} \mathrm{C}\left(535^{\circ} \mathrm{F}\right)$, the bypass valve is actuated, sending the Caloria to the top of the storage tank. Figure 6 indicates that a large quantity of cool Caloria was pumped into the top of the storage tank during the day. The only possible explanation is that the bypass valve was allowing a portion of the $204^{\circ} \mathrm{C}\left(400^{\circ} \mathrm{F}\right)$ Caloria that was circulating through the collector field to leak past it into the top of the storage tank. Prior to running this test, it was known that some leakage was present, but the leakage was believed to be small. Now it is estimated to be $0.6 \mathrm{l} / \mathrm{s}(10 \mathrm{gal} / \mathrm{min})$, which 


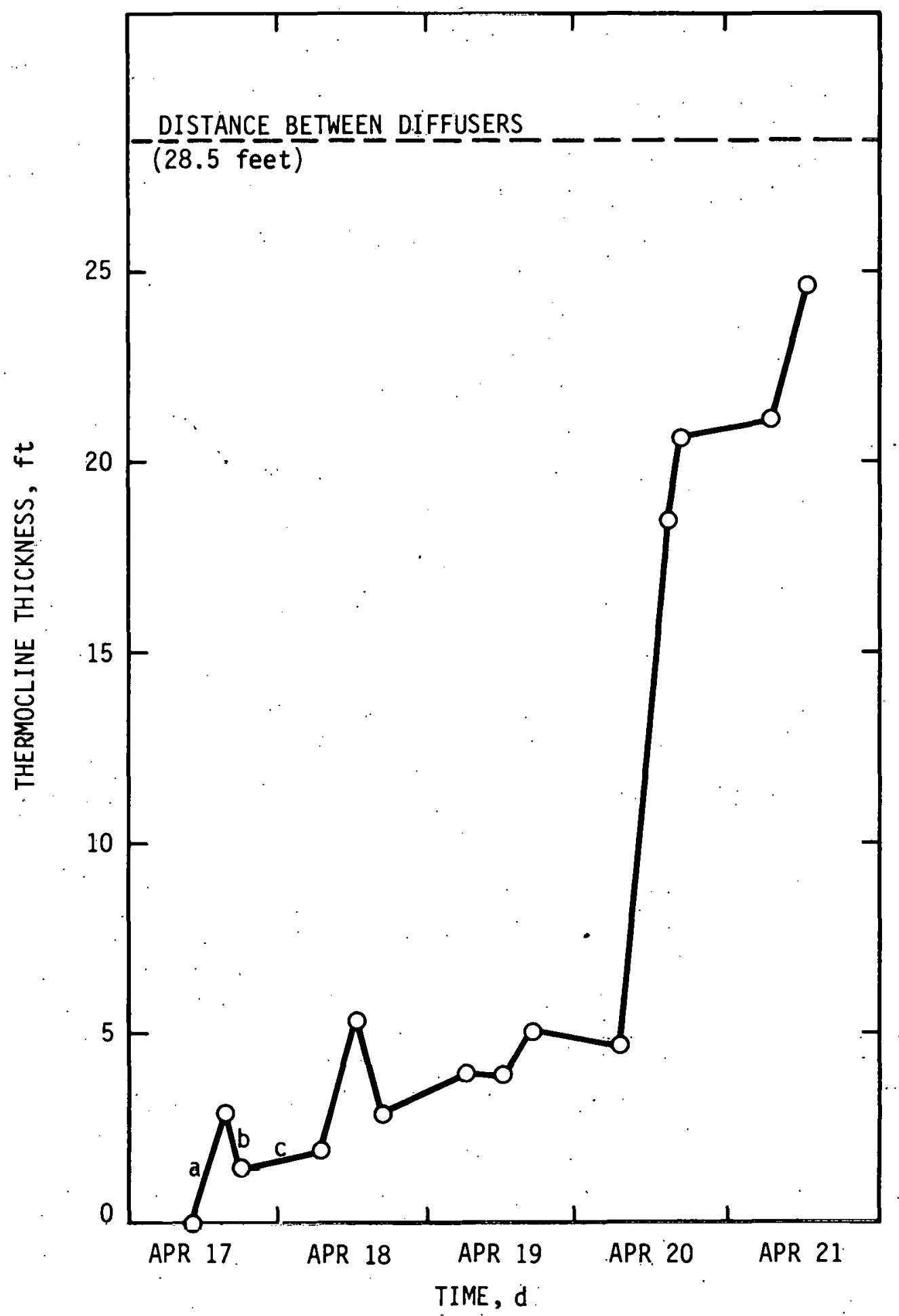

Figure 2. Thermocline Thickness vs. Time 


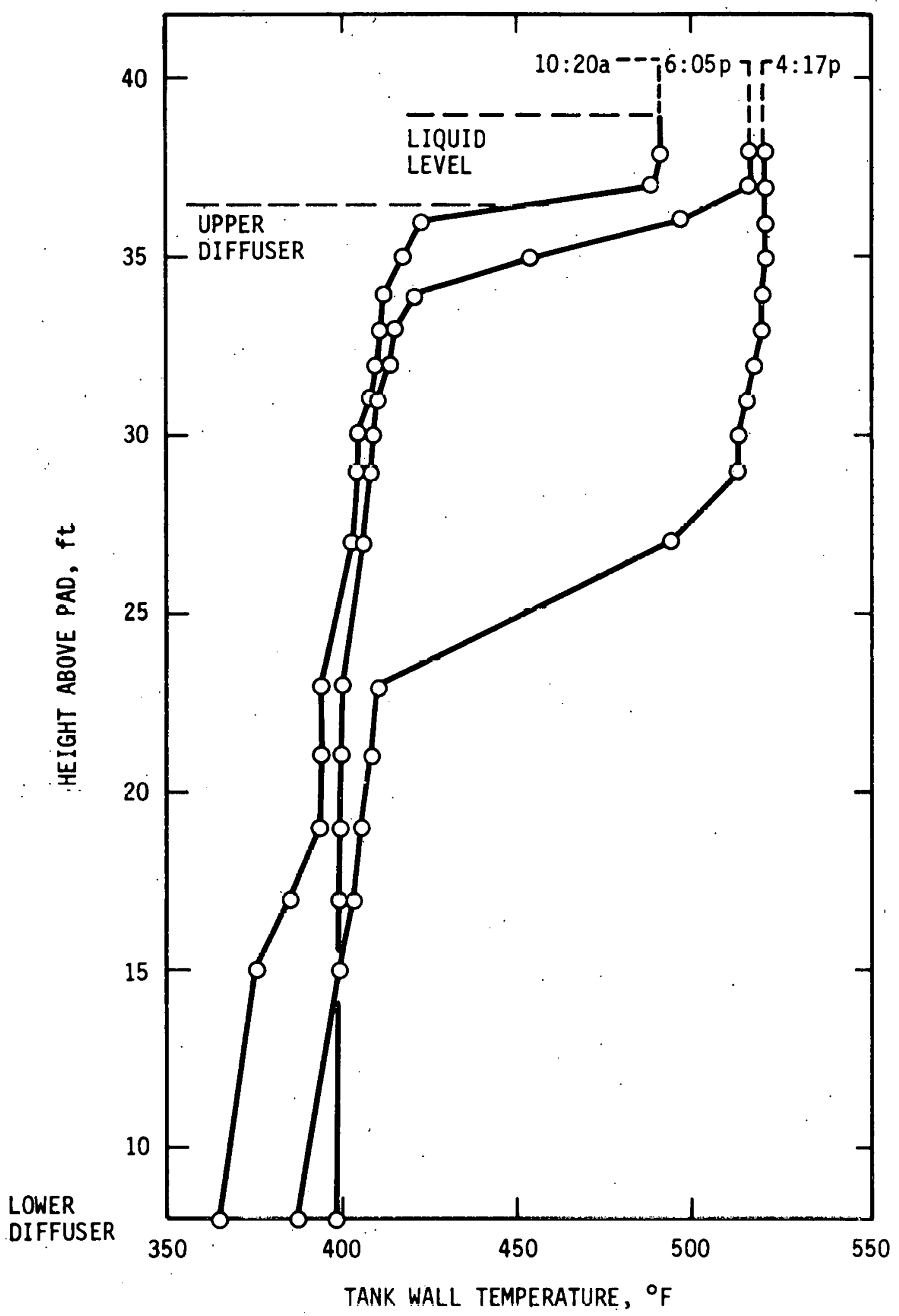

Figure 3. Thermal Storage Tank Temperature Profile, 17 April 1980 


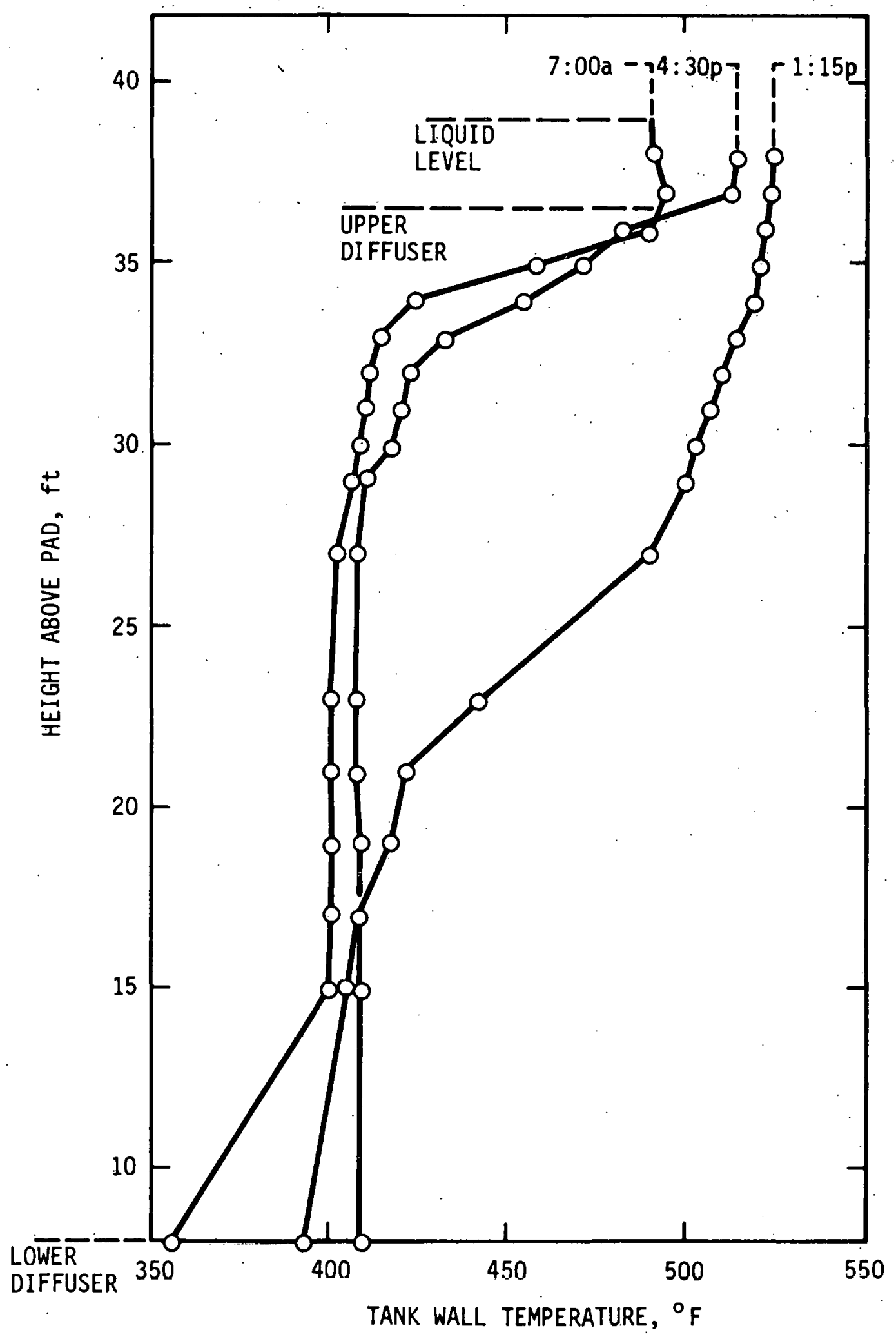

Figure 4. Thermal Storage Tank Temperature Profile, 18 April 1980 


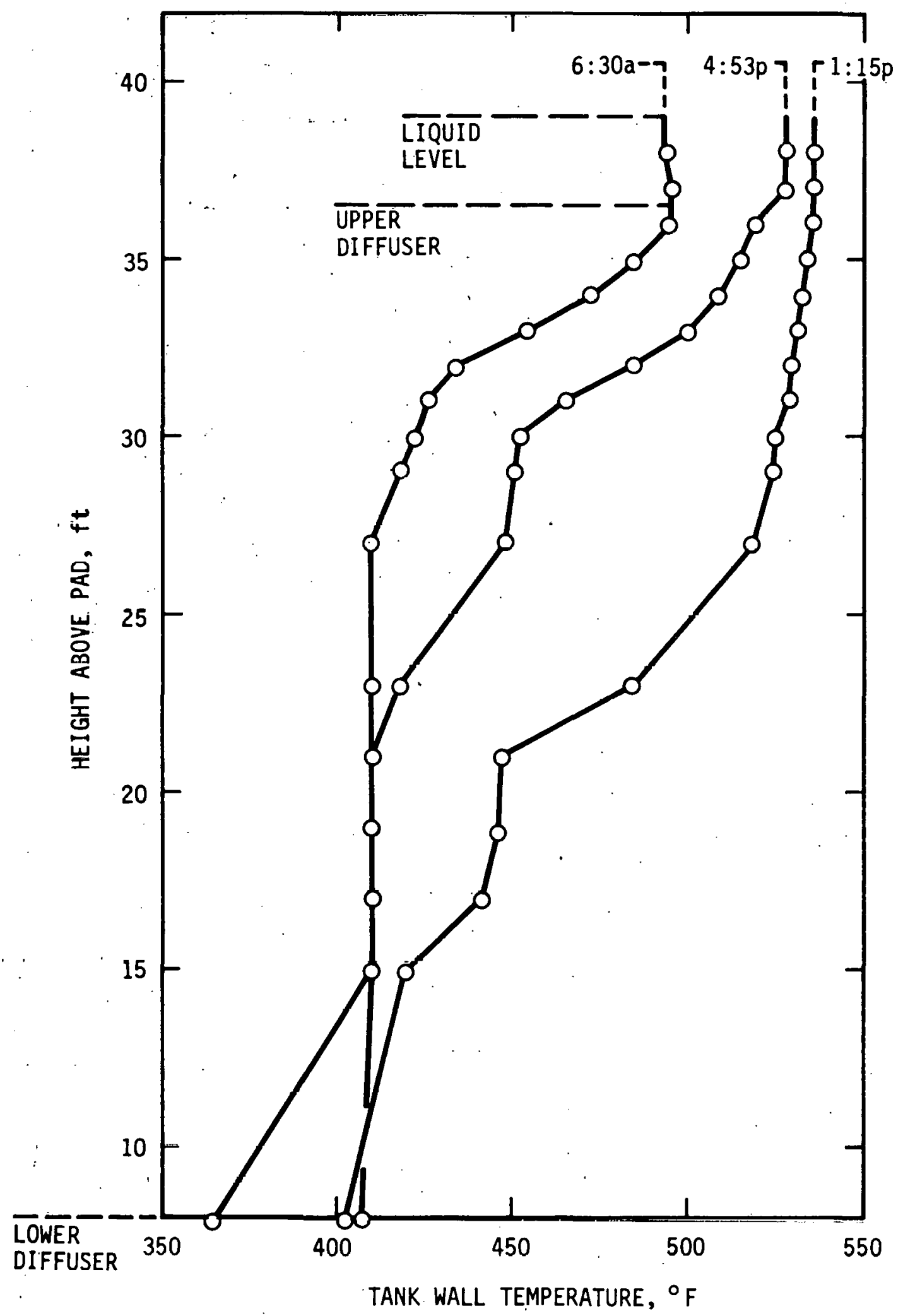

Figure 5. Thermal Storage Tank Temperature Profile, 19 April 1980 


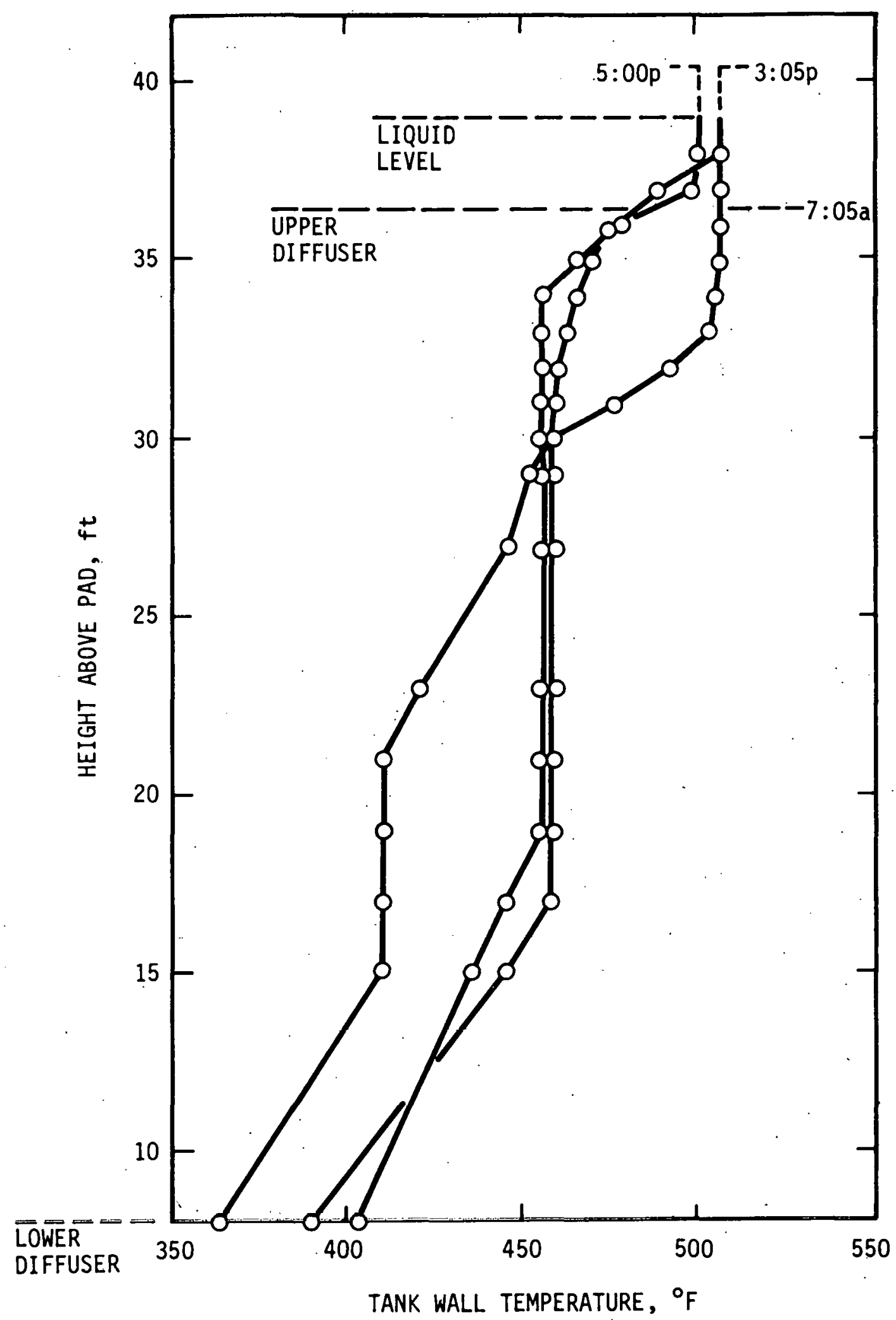

Figure 6. Thermal Storage Tank Temperature Profile, 20 April 1980 


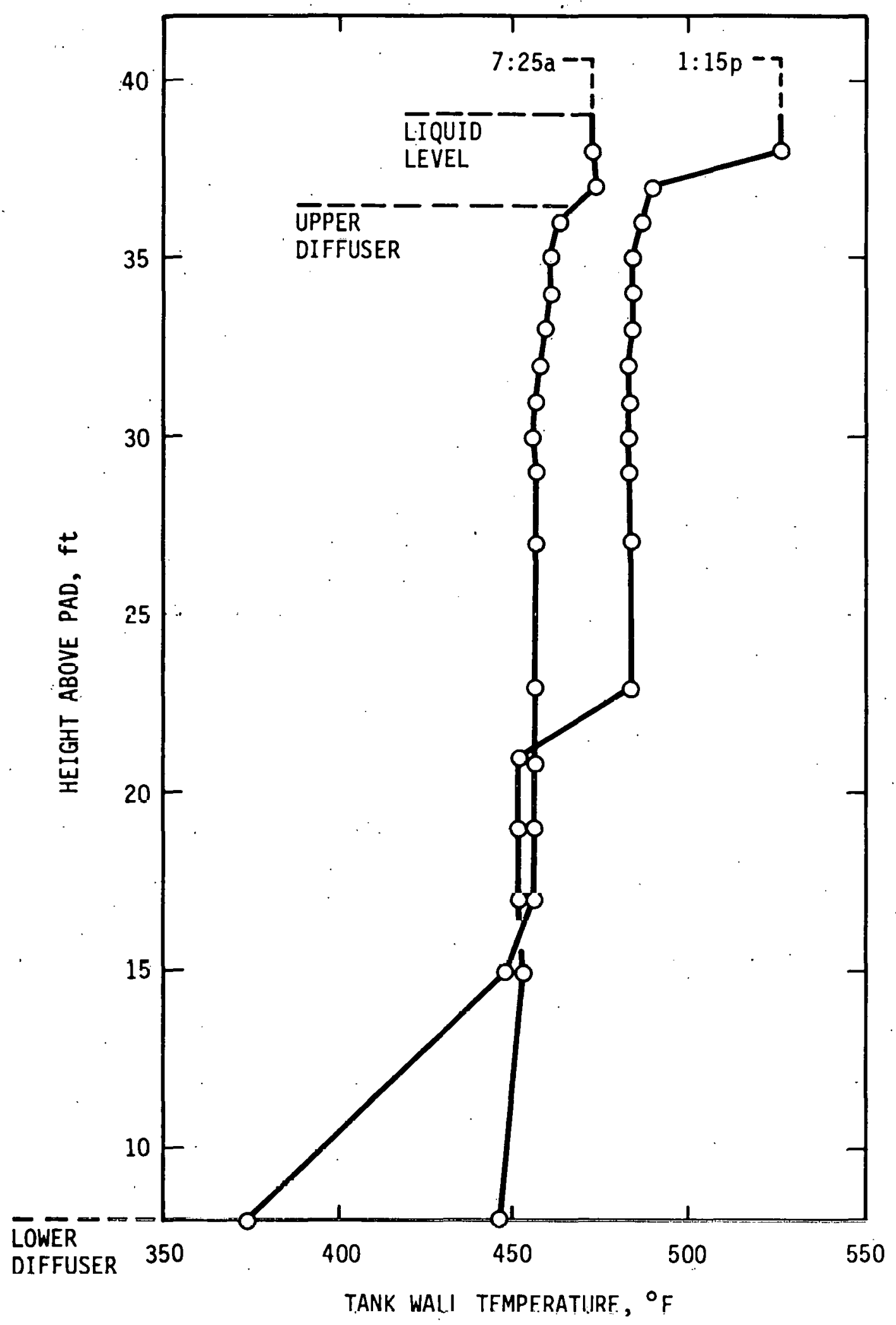

Figure 7.: Thermal Storage Tank Temperature Profile, 21 April 1980 
is large compared to the $1.9-\ell / \mathrm{s}(30-\mathrm{gal} / \mathrm{min})$ flow rate during circulation.

The significant leakage through the bypass valve also occurs during collector field warmup each morning. This not only introduces cool Caloria to the top of the storage tank but also extends the warmup time of the collector field.

The fifth day of the test was also cloudy; however, some heat was added to storage.

\section{Discussion}

The thickness of the thermocline would probably have remained relatively small throughout the course of the test had it not been for the leaking bypass valve. It is the author's opinion that a three-way diverting valve with a conventional spool would be a better choice than the three-way ganged butterfly diverting valve used at coolidge.

The lower of the two diffusers in the top of the storage tank was not utilized during this test series, which demonstrates that the system will function this way. It is the author's opinion that the test results would look less favorable if the lower diffuser had been used for adding Caloria to the top of storage in keeping with the system's original design. The conclusion is that future designs need not include a second diffuser at the top of a thermocline storage tank. 
- Blunk Page

132 


\section{EXAMPLES OF THERMOSIPHONING AT COOLIDGE, ARIZONA}

by

R. W. Harrigan

During a recent trip to the Coolidge Deep Well Irrigation Project, several clear examples of thermosiphoning were noted. While no quantitative measurements were made (temperatures were usually recorded as cold, warm, hot, and very hot to the touch), the qualitative results are worth discussing.

The piping around the mixing and thermocline tanks is shown in Figure 1. During the observations of thermosiphoning, there was no flow in the collector field since the day was overcast and rainy. The three-way valve, $v_{1}$, in the collector return line was open to the mixing tank, and there was hot fluid in the mixing tank from the previous day. A thermometer, $T$, in the collector return line near $V_{1}$ indiçated the fluid at that point was about $193^{\circ} \mathrm{C}\left(380^{\circ} \mathrm{F}\right)$.

The first indication of thermosiphoning was in the fossil-fuel heater line. Valve $V_{3}$ was open, and, even though there was no pumped flow in this line, the uninsulated pipe at point $P_{1}$ (where the insulation stopped) was quite hot. In addition, only the top part of the uninsulated pipe was hot (too hot to keep your hand on $i t$ ); the bottom was cold to the touch. The top of the uninsulated pipe cooled gradually with distance from $P_{l}$, with both the top and bottom of the uninsulated pipe reaching ambient temperature in about 3.048 to 4.57 metres (10 to 15 feet). The large temperature difference between the top and bottom of the pipe at point $P_{1}$ is even more remarkable since a light misty rain was falling on the pipe to cool it. 


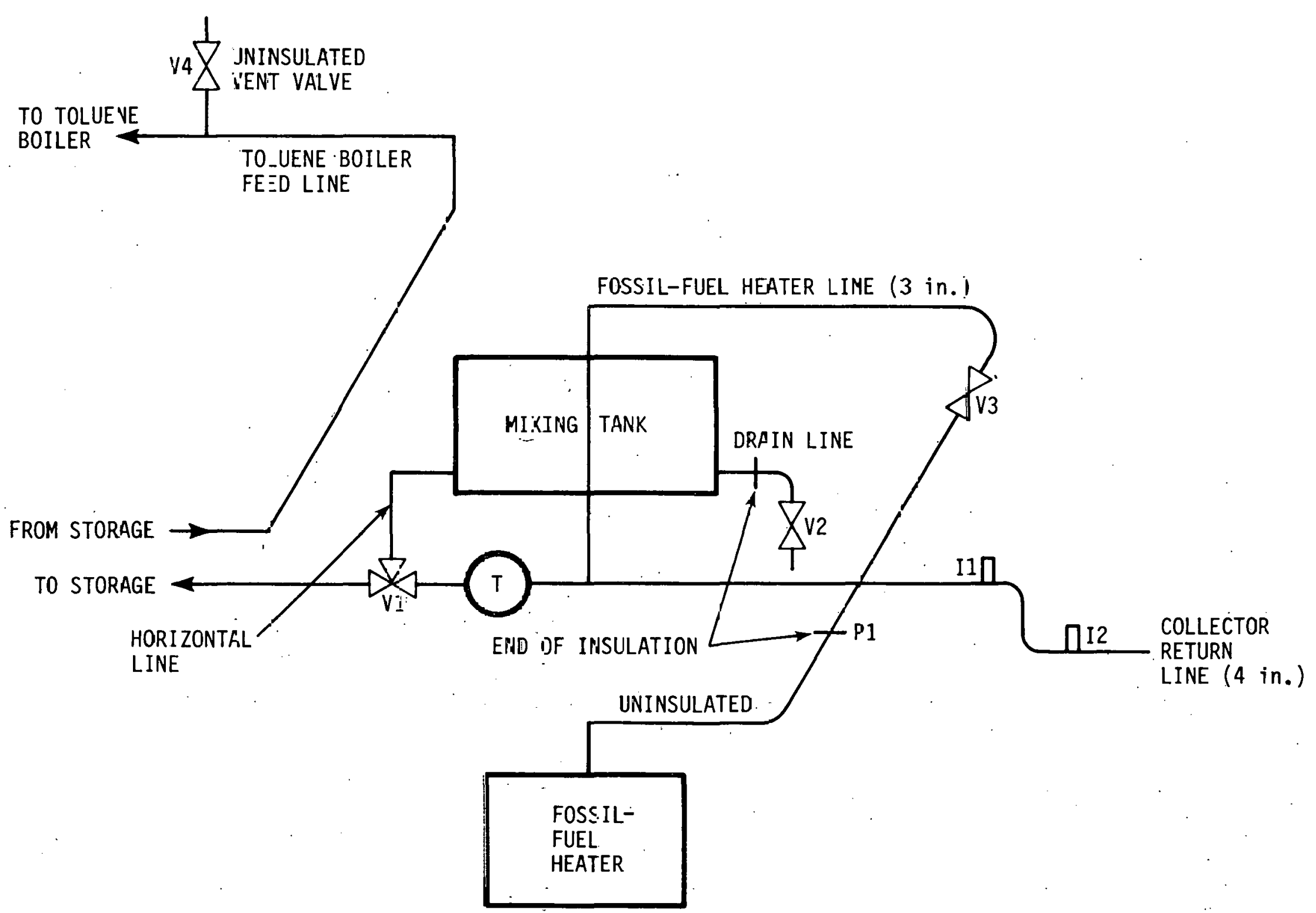

Figure 1. Mixing "Tank and Thermo:line Tank Piping 
To further check" on thermosiphoning, valve $v_{3}$ wäs then closed. The uninsulated pipe at $P_{1}$ cooled to ambient over several hours. When $V_{3}$. was subsequently opened, $P_{1}$ again heated up as above, indicating the presence of thermosiphoning.

In order to see the effect of a drop in elevation of a hot line on thermosiphoning, points $I_{1}$ and $I_{2}$ were examined. $I_{1}$ and $I_{2}$ are instrumentation posts welded directly to the collector return 1 ine. Since there was no flow through the collector field, it might be expected that $I_{1}$ and $I_{2}$ would be cool. However, it was found that $I_{1}$ was quite warm to the touch, while $I_{2}$ was cold. This indicates the possibility of thermosiphoning from the mixing tank to $I_{1}$. In addition, $I_{2}$ being cold indicates that the drop in elevation between $I_{1}$ and $\mathrm{I}_{2}$ (about 0.3048 metre [12 inches]) is enough to stop thermosiphoning:

Another interesting observation was made at the mixing tank drain line. This line comes from the bottom of the mixing tank and is insulated only part way along its length, so that the elbow in the line is exposed. The distance from the elbow to valve $V_{2}$ is about 76.2 to $101.6 \mathrm{~mm}$ ( 3 to 4 inches). The elbow in the drain line was very hot, too hot to touch, while valve $v_{2}$ was cold. This indicated good thermal communication between the mixing tank and the elbow in the drain Iine but poor thermal communication between the elbow and valve. This would be expected if thermosiphoning were the major thermal transport mechanism. As a verification of the phenomenon observed, valve $v_{2}$. was opened to drain hot fluid through the line to heat up the entire.line. After cooling for about an hour; the valve was again cold and the elbow was very hot.

An analogous situation, but with the valve inverted from $v_{2}$, was found in the line supplying hot oil from storage to the toluene boiler. Valve $V_{4}$, which extended about. 101.6 to $152.4 \mathrm{~mm}$ (4 to 6 inches) above the insulated toluene boiler feed line, was found to be very hot to the touch. The fluid in the toluene boiler feed line was hot because the turbine was in operation, demanding loot fluid from storage. 
While no quantitative conclusions are possible at this stage, it seems obvious that very significant amounts of thermosiphoning are occurring and that relatively small dips in line elevation may be sufficient to stop at least some thermosiphoning. 
EQUIPMENT PROBLEMS AND SOLUTIONS FOR THE
COOLIDGE, ARIZONA, SOLAR IRRIGATION FACILITY

Introduction

The Coolidge Solar Irrigation Facility began operation in October 1979. Since 1 January 1980, the plant has operated daily except for a period from mid-February to early March. On 12 February, a fire in the collector system pump shroud area necessitated pump removal and repair, and inclement weather delayed repair efforts. Other problems have affected plant operation for a short period, caused one system to be inoperative, or resulted in reduced performance. This report describes the primary problems encountered during the year and lists solutions and potential solutions to the problems.

\section{Collector Tracking Units}

Collector tracking systems have required considerable attention to assure proper operation. On a typical operating day, from 1. to 3 of the 48 tracking units needed maintenance attention or repair. Malfunctions can be categorized as moisture-related, sensor photodiode, and control circuitry problems.

Moisture in sensor windows and sensor cable connectors has caused incorrect focusing and searching. Moisture collected through condensation or entered during rainfall. Disassembly and wiping or air arying corrected the tracking problem. Sealing the connectors appears to have remedied the connector moisture problem at coolidge. . Sensor case design has been modified to minimize moisture entrance.

Photodiode arrays in many sensors have cracked, resulting in inaccurate tracking. Photodiodes now are being encased in a different 
material, and replacement of sensor assemblies is being proposed by the manufacturer.

Sensor control circuitry failures also have caused some tracking problems. Some failed relays and resistors have been replaced in the field. Other control boards were returned to the manufacturer for repair.

\section{Collector Drive Motors}

Five drive motors failed in February and March and two others failed later because rear shaft bearings had broken loose from the cases. The motors were repaired by à. 'l'ucson shop and by superior Electric, the manufacturer. The failures were attributed to manufacturing problems; required corrections will be made by the manufacturer: A contributing circumstance was an apparent lack of testing with motors mounted in the collector system application orientation.

\section{Flexible Hoses}

The bending motion required of flexible hoses is variable. Some hoses are bent into an "S" shape, and, in many groups, nonplanar bending is necessary.. Because compound bending hastens the failure of the hoses, improved means for interconnecting collector groups are being sought.

Thirty-six of 96 flexhose covers have failed to date, and several flexible hose insulation covers have become detached from collector attachment points. To limit the incidence of failure, wider attachment rings and different clamps are being evaluated.

Nearly all flexible hose covers located at the north end of collector groups have deteriorated due to sunlight reflection and have subsequently torn in flexure. A sun shield, which prevents reflection of concentrated sunlight onto the flexhose cover, is being tested at Coolidge. Installation of shields throughout the collector field is being proposed by the manufacturer. 


\section{Receivers}

Collector receiver tube black chrome coatings have deteriorated visibly. In most collector loops, deterioration is substantial in the two highest temperature groups, moderate in the two medium-temperature groups, and slight in the two groups experiencing the lowest temperatures: An evaluation of the effect of receiver surface changes on performance is contemplated.

\section{Receiver Covers}

Inadequate end sealing of collector receiver glass covers has permitted dust intrusion, which is most apparent with tubes at the ends of collector groups. There, sunlight reflection is apparent. One collector group at Coolidge has been retrofitted with a modified insulation cover that abuts the glass receiver cover and reduces dust intrusion. The insulation appears to have minimized dust intrusion since installation. Further evaluation will precede a possible complete retrofit.

\section{Receiver Insulation}

Receiver tubes at collector group ends rotate within stationary foam glass insulation covers. The relative motion has increased the foam glass insulation interior diameters, causing the covers to sag. Another insulation material is recommended where relative motion is substantial.

\section{Pump Leakage and Fire}

Both pumps moving Caloria through the collector system and to the vaporizer have experienced excessive leakage from shaft seal areas. Vaporizer pump leakage began during the initial operation and continues despite seal replacement and other repair efforts performed in late February. The initial leakage apparently was caused by metal filings or slag from construction.

Collector system pump leakage developed over a period of operation, becoming substantial by February. Late on 12 February; a fire 
occurred in the pump shroud area, causing system shutdown. Hot Caloria apparently autoignited near the leakage location. New pump bearing and seal and motor electrical cable were required. Inclement weather, replacement part procurement, and repair efforts halted plant operation for 23 days.

It was determined that the pump seal area should be purged by inert gas flow or cooled during operation. A carbon dioxide purging system, using refillable cylinders and a flow control valve, was connected to each pump. Seal replacement and carbon dioxide usage have reduced, but not eliminated, collector system pump leakage. Another seal, of a different material, is being acquired for evaluation.

The fire caused a reevaluation of plant operation. Leak stoppage' has become a high-priority task. Emergency procedures have been developed to respond to fire, leakage, and personnel injury incidents. Additional fire extinguishing and first aid equipment was procured and strategically located.

\section{Pneumatic System Leakage}

On many occasions, excessive air usage by pneumatic valve actuators resulted in system shutdown after extended operation. Additional air compressor capacity was obtained, one actuator was replaced, connections were tightened, and other actuators were, serviced. Air leakage has decreased but is still a problem. However, the additional compressor capability has made air loss a problem which does not threaten operation.

\section{Storage Tank Leakage}

Caloria continues to leak from flanged manhole covers on the side of the main storage tank. Securing bolts have been retightened periodically, but leakage soon begins again. The primary effects are insulation contamination and dirty appearance. 


\section{Flow Control Valves}

Valves installed to control the flow of Caloria to the various collector loops soon became clogged with pipe contaminants. The valve interiors were removed for repair and never reinstalled. The oil flow rate to each of the eight collector loops is apparently nearly equal as judged by outlet temperature measurements. Thus, the valves are not deemed necessary.

Three-way, butterfly-type valves, used to direct Caloria to alternative locations, have experienced leakage into the closed path. Actuator replacement has decreased leakage.

External leakage has occurred from valve stems and flanged connections of many remotely, and manually, actuated valves. The result is unattractive and, with insulation contamination, potentially a fire danger. Flange bolts have been retightened; repacking of some valve stems is planned.

\section{Toluene Leakage}

Some toluene replenishment has been required due to losses from connections and from the separator tank. Valve packing has been tightened at two locations, eliminating leakage. Separator tank loss is believed to be normal.

\section{Organic Rankine Cycle (ORC) Vacuum Leakage}

The vacuum in the power conversion system decreases from the. 0.71 -metre $(28$-inch) working level to perhaps 0.38 metre ( 15 inches) overnight. In 48 hours, the vacuum drops to nearly zero. Leakage tests for pressurized inert gas and retightening of connections reduce the vacuum loss for a short time. The effect of vacuum loss is to increase vacuum pump usage.

\section{Vaporizer Level Sensor}

The sensor which measures toluene level (or quantity) in the vaporizer gives erroneous values. Becaucc thcsc values are used to 
control the flow of toluene, visual observation of the sight glass level and operator control of the level are required. Replacement with a different type of level sensor is scheduled for september 1980.

\section{Turbine Gearbox Lubrication System}

Gearbox lubrication pressure became too small during extended operation on a hot day, resulting in turbine shutdown. The input pressure can be adjusted; upward adjustment of the lubrication system pressure has eliminated the problem.

\section{Generator Synchronization}

The control system causing the generator to produce electricity compatible with the utility grid systell llalluncliurid in late sping. Operator control of synchronization during startup thus was required for about 2 months.' A malfunctioning control system component was. identified and replaced and the system readjusted by onsite technicians with telephone direction from the manufacturer.

\section{Cooling Tower Pump}

Cooling tower pump outage occurred on several occasions, resulting in turbine shutdown. The pump overload sensor.was replaced; outage has not recurred.

\section{Toluene Contamination}

Caloria was inadvertently added to the toluene supply during routine replenishment, making the supply about $10 \%$ oil. Reduced power system performance, particularly vaporizer performance, and discoloration of toluene were the problem symptoms. Contamination by Caloria then was suspected and later was confirmed by láboralory tests.

The Caloria gradually was removed from the vaporizer, which acted as a distillation unit. The process involved ORC operation--during which time separation occurred, Caloria removal from the vaporizer, and toluene replenishment. 


\section{Measurement Devices}

A relay in the direct insolation monitor (DIM) failed in June, causing failure of additional relays. While awaiting repair, operator actuation of the DIM control was required. The faulty relay was identified and repaired onsite.

Flow meter measurements were doubted, so meters were recalibrated. Periodic recalibration may be required.

High-gain transmitters of insolation, temperature, and flow measurements have failed on three occasions. Repair required shipment to the manufacturer.

Resistance temperature detectors (RTDs) used for temperature measurement at many plant locations have failed or exhibit signs of impending problems. Two indications of potential failure are cracked terminal strips and corroded wire sensors.

\section{Summary}

A number of maintenance problems occurred during the year. Two problems, collector system pump failure and pneumatic control system air pressure losses, resulted in system shutdown. However, only pump repair caused extended shutdown.: Other problems, such as vaporizer level sensor and collector tracker system malfunctions, increased operational requirements and may have reduced plant performance. These and other problems have led to improvements which have increased plant reliability and performance and decreased operational requirements. Second-year operation will evaluate improvements and provide further testing of original equipment. 
Blank Page

144 
OPERATING COSTS FOR THE COOLIDGE, ARIZONA, SOLAR IRRIGATION FACILITY

The Coolidge Solar Irrigation Facility has been operated on a daily basis during the hours of solar energy availability. One or more persons have been in attendance during all plant operations. Plant personnel have attended to operational tasks, repair and maintenance jobs, data gathering activities, visitor information responsibilities, and plan improvement efforts. The operational requirements, quantified operator time, and operational supply costs are difficult to separate from other requirements. However, an estimate is presented below.

Operators initiate plant operation by opening a safety valve, preventing oil loss from the storage tank. Then they turn on the collector system. Daily efforts include monitoring the operation and assuring that operation is normal. Power conversion system operation has required continued observation and some manual control. Manual control of the separator coolant flow valve is required during startup preparations. Inspection of the storage tank nitrogen supply, pump $\mathrm{CO}_{2}$ supplies, and cooling tower water treatment chemical supply is required daily; replenishment is accomplished as needed. Cooling water quality must be analyzed on alternate days. Plant shutdown and site security require additional operating time. Collector washing was categorized, arbitrarily, as recurring maintenance.

It is estimated that these operational activities have required 30 hours of operator effort per week, i.e., 4 to 5 hours per day. Summer requirements have been greater; winter requirements, smaller. 
Materials and supplies required for operation include the cooling tower water supply, water treatment chemicals, carbon dioxide to purge pump seal areas, and nitrogen to blanket Caloria in the storage tank. Another operating expense is electrical energy for cooling the building housing electronic control systems. The latter expense depends directly on weather conditions. Many other requirements are related to amount or length of operation, which also varies seasonally.

The list below provides an estimate of operational costs: $\$ 78$ per week in summer, $\$ 26$ per week in winter.

Weekly Costs of Plant Operation

$\begin{array}{lcr} & \frac{\text { Summer }}{\$ 20} & \frac{\text { Winter }}{\$ 5} \\ \text { Water (municipal) } & 35 & 10 \\ \text { Water treatment } & 5 & 5 \\ \text { Nitrogen } & 8 & 6 \\ \mathrm{CO}_{2} & 10 & -- \\ \text { Electricity (cooling. est.) } & \$ 78 & \$ 26 \\ \text { Total cost per week } & & -\end{array}$

Power converoion Jyatcm opcration will be nearly automatic afLí installation of a replacement vaporizer level sensor in september 1980. Planned installation of a remotely actuated safety valve will further automate operation. The labor reduction will be evaluated during the upcoming year.

Material costs also might be reduced somewhat. For example, less $\mathrm{CO}_{2}$ would be used with niqhttime shutdown, but manual valve operation probably would be required. Materials usage will be monitored carefully and reduced where possible during the 1980-81 operating period. 
RECURRING MAINTENANCE REQUTREMENTS FOR THE COOLIDGE, ARIZONA, SOLAR IRRIGATION FACILITY

\section{Introduction}

The amount of time devoted to recurring maintenance tasks and the cost of supplies and replacement materials for those efforts have been recorded since late January 1980. The attempt to quantify recurring maintenance needs required identification of those activities. Maintenance tasks, as distinguished from repair efforts, are defined to be recurring, expected efforts. Repair activities are those required due to equipment failures or accidents and usually occur unexpectedly. Daily operating requirements also were distinguished from maintenance requirements. Operational activities include inspection and replenishment of condenser cooling tower water treatment chemicals, Caloria storage tank nitrogen supply, and Caloria pump $\mathrm{CO}_{2}$ supplies.

\section{Tasks}

Recurring maintenance requirements of the collector subsystem include cleaning the reflector surfaces, the receiver glass cover tubes, and the tracker sensor windows as required, greasing bearings. and checking drive unit gearbox oil levels (replenishing if required) on a bimonthly basis, testing electric drive motors, also bimonthly, and inspecting and adjusting collector module receiver tube and sensor alignment quarterly. Periodic, perhaps monthly, inspection of the fluid transfer system for leakage or other changes is recommended. Valve stems should be lubricated on a periodic basis. Caloria transfer system condensate must be drained weekly.

In the power conversion subsystem, monthly requirements include greasing bearings and inspecting start pump and turbine gearbox oil 
levels (adding as needed), checking toluene level, and inspecting bolts and fittings (tightening, if required). Toluene and gearbox oil filters, as well as gearbox oil, must be changed annually. The cooling tower requires periodic cleaning, perhaps monthly. A vacuum leakage inspection also should be conducted periodically.

Air compressors require monthly inspection and periodic change of lubricant and periodic cleaning of air filters. Pneumatic system leakage should be tested monthly and fittings tightened as required. Electrical relays should be dusted, perhaps by blown air, on a periodic basis. Other recurring tasks include pyranometer adjustment and equipment calibration. Site activities include cleanup and pest (insect, animal, and weed). control efforts. Safety shutdown devices requiré monthly testing; fire extinguishers require semiannual testing.

$\underline{\text { Time }}$

Recurring maintenance tasks have required a recorded effort averaging 17.6 hours per week. The collector system required about 8. 0 hours, the fluid transfer system 4.8 hours, and the power conversion system 4.8 hours. The effort varied substantially from week to week, as shown in rable 1. The differences can be attributed to labor availability as well as task requirements.

\section{Materials}

Collector system maintenance supplies include lubricants for bearings and gearboxes, materials for cleaning collector surfaces, fuses, and relay cleanser. The fluid transfer system requires degreaser for cleaning operations and some lubricant for the valves. Power conversion system requirements include pump lubricant, toluene filter, and gearbox lubricant and filter. Some replacement toluene and Caloria may be required, primarily depending on leakage. Other items needing periodic replacement include power supply filter and test panel light bulbs. Air compressor filter and oil must be changed periodically. Herbicide is required for adequate grounds maintenance; some pesticide is required for control building use. 
Table 1

An Estimate of Recurring Maintenance Requirements for Collector, Fluid Transfer and Storage, and Power Conversion Systems

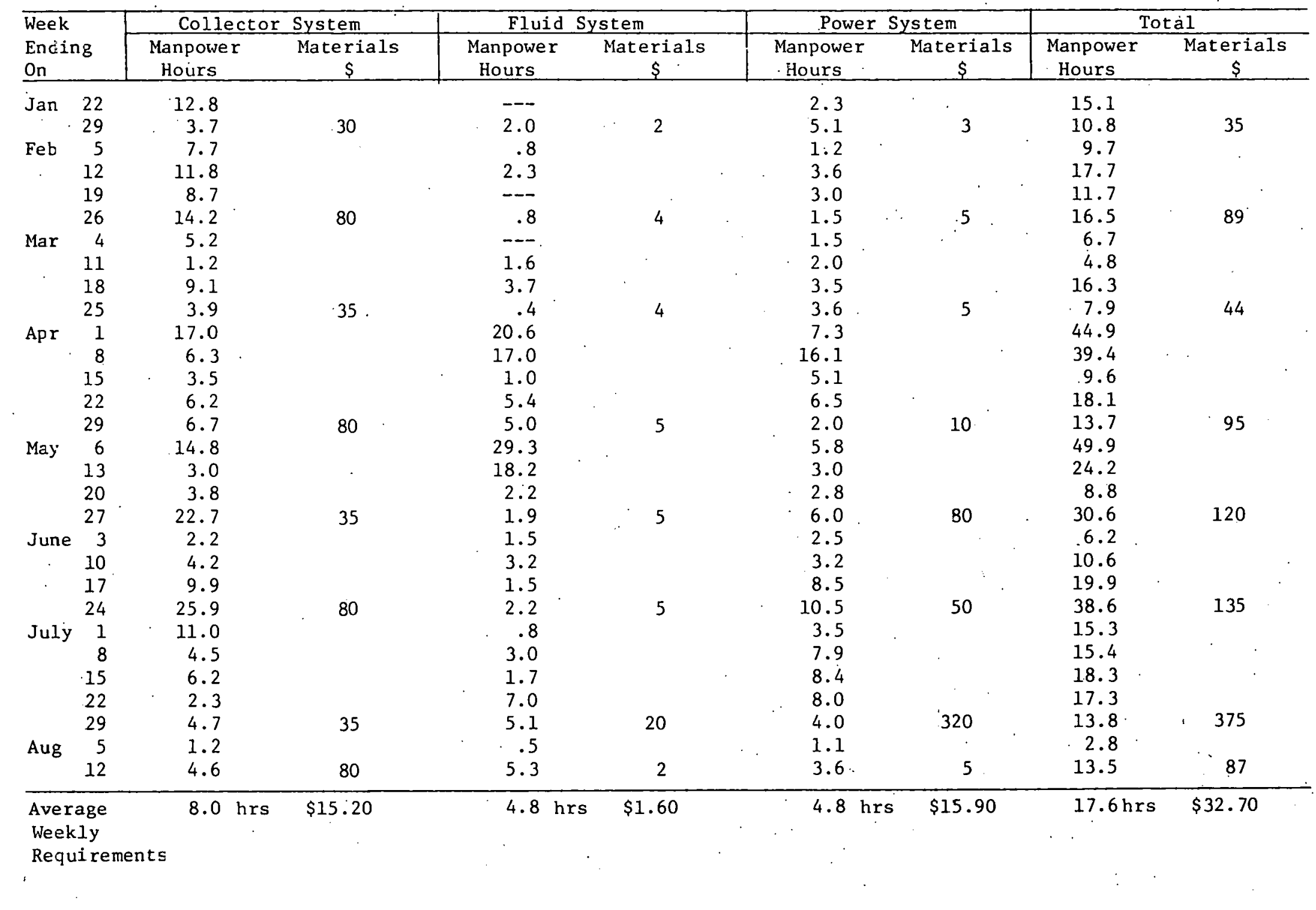


Some maintenance items are used for periodic servicing and replacement, while others are used as required. Service intervals vary from daily to annually. Table 1 contains an estimate of the cost of maintenance materials during the 8-month period mid-January to midAugust 1980. Since an inexact usage schedule exists, costs arbitrarily were apportioned to the last week of each month. Lubricant was the largest collector system expense; a $0.21-m^{3}$ (55-gallon) drum of toluene and gearbox lubricant and filter were the major power conversion system costs. The average weekly expenditure for recurring maintenance materials was $\$ 32,70$. At this rate, the annual cost would be about $\$ 1700$.

\section{Data Improvement}

Classification of certain tasks or costs by operation, maintenance, repair, or other cateqory was difficulț. In some cases, categorization may have been arbitrary. Maintenance tasks were being identified throughout the year, methods for performing some duties had to be learned on-the-job, and regular task schedules are still being developed. Thus, the information presented in Table 1 is only a first estimate of recurring maintenance requirements. Additional maintenance effort data will be recorded during the upcoming year.

\section{Eummaey}

Recurring maintenance requirements of the Coolidge Solar Irrigation Facility were estimated to be 17.6 man-hours per week and $\$ 1700$ materials cost per year. Continued data gathering efforts will attempt to improve this estimate. 
CONSTRUCTION COSTS FOR THE COOLIDGE, ARIZONA, SOLAR IRRIGATION FACILITY

The original system design for the Coolidge, Arizona, Solar Irrigation Facility specified $4548.5 \mathrm{~m}^{2}\left(48,960 \mathrm{ft}^{2}\right)$ of collector aperture area. The installed collector field consists of $2140.49 \mathrm{~m}^{2}$ $\left(23,040 \mathrm{ft}^{2}\right)$. The field reduction was necessitated by rising contract costs. Before the field reduction decision was made, the site had been prepared for the full-size field. The preparation included foundations for collectors and pipe supports. The costs presented here have been adjusted to exclude the expenses associated with site preparation and foundation installation for the second half of the collector field.

The total cost incurred for the design, procurement, construction, and startup of the facility over the 2-year period ending 30 September 1979 was $\$ 5,512,000$. Of this amount, approximately $\$ 2,023,000$ was used on labor costs incurred by Acurex Corporation and $\$ 3,489,000$ went to subcontracts, equipment purchases, etc.

The same system if built today would cost significantly less-only $\$ 2,551,000$, owing to nonrecurring costs of labor, engineering, management, data acquisition equipment, and experience with similar systems.

Table 1 presents a cost breakdown and compares the actual system cost with the recurring costs of the same system. In Table l, the item called "Installed Collectors" consists of collector hardware, installing the collectors on the foundations, and plumbing the collector field. The item entitled "General Construction" consists of site preparation, collector foundations, mechanical contract work on 
the site, electrical contract work on the site, and the total costs of the insulation for the pipelines and tanks on the site. 
Table 1

Breakdown of Construction Costs

Item
Installed Collectors
General Construction
Site Preparation and
Foundations
Mechanical Contract
Electrical Contract
Insulation Contract

Building

ORC

Storage Subsystem

Controls and Data

Acquisition Equipment

Design and Field Support

Management

Initial Operational

Expenses

System Completion

Water Supply, ORC Shelter, Emergency Generator, ORC Lube System

System startup and Dedication

Safety Improvements

Fire Hydrant, etc.
Actual Cost of

Current System

\section{$\$$}

810

833

(184)

(346)

(230)

(69)

$$
50
$$

1068

$209^{\prime}$

3.58

1493

530

$\$ / f t^{2}$

35

36

50

2

.46

9

16

65

23

65

3

10

0.5
Recurring Costs of Same system

$\begin{array}{cc}\$ 000 & \$ / \mathrm{ft}^{2} \\ 530 & 23 \\ 645 & 28\end{array}$

50

2

650

27

150

7

150

7

150

7

200

9

Total

$\$ 5512$

$\overline{\$ 239}$

$\overline{\$ 2551}$

$\overline{\text { \$11 }}$ 
DISTRIBUTION :

TID-4500-R66, UC62 (268), 11/80

AAI Corporation

P.O. Box 6787

Baltimore, MD 21204

Acurex Aerotherm (20)

485 Clyde Avenue

Mountain View, CA 94042

Attn: J. Vindum

Argonne National Laboratory (3)

9700 South Cass Avenue

Argonne, IL. 60439

Attn: K. Reed

W. W. Schertz

R. Winston

BDM Corporation

1801 Randolph Street

Advanco Corporation

999 N. Sepulveda Blvd.

Suite 314

El Segundo, CA 90245

Attn: B. J. Washom

Alpha sulaido

1014 Vine Street

Suite 2230

Cincinnati, OH 45202

Albuquerque, NM 87106

Attn: T. Reynolds

Battelle Memorial Institute Pacific Northwest Laboratory P.O. Box 999

Rit:hland, WA 99352

Attn: K. Drumheller

Bechtel National, Inc.

P.O. Box 3965

American Boa, Inc.

Suite 4907, One World

Trade Center

New York, NY 10048

Attn: R. Brundage

Anaconda Metal Hose Co.

698 South Main Street.

Waterbury, C'I' 06720

Attn: $W$. Genshino

Applied Concepts Corp. .

P.O. Box 2760

Reston, VA 20090

Attn: J. S. Hauger

Applied Solar Resources, Inc. 490 East Pima

Phoenix, AZ 85004

Attn: W. H. Coady

Arizona Public Service Co.

Box 21666 MS 1795

Phoenix, AZ 85036

Attn: Dr. B: L: Broussard

50 Beale Street

San Francisco, CA 94119

Attn: E. Y. Lam

Black and Veatch (2)

P.O. Box 8.405

Kansas City, MU 54114

Attn:' Dr. J. C. Grosskreutz

D. C. Gray

Boeing Spare Senter (2)

M/S 86-01

Kent, WA 98131

Attn: S. Duzick

Ä. Lunde

Boomer-Fiske, Inc.

4UUU s.. Princetur

Chicago, IL 60609

Attn: C. Cain

Budd Company

Fort Washington, PA 19034

Attn: W. W. Dickhart

The Budd Company

Plastic R\&D Center

356 Executive Drive

Troy, MI 48084

Attn: J. N. Epel 
DISTRIBUTION (Continued)

Carrier Corp.

Energy Systems Div.

Summit Landing

P.O. Box 4895

Syracuse, NY 13221

Attn: R. A. English

Compudrive Corp.

76 Treble Core Road

N. Billerica, MA 01862

Attn: T. Black

Cone Drive

Division of Excello Corp.

P.O. Box 272

240 E. 12 S.t.

Traverse City, MI 49684

Attn: J. E. McGuire

Congressional Research Service

Library of Congress

Washington, DC 20540

Attn: H. Bullis

Corning Glass Co. (2)

Corning, NY 14830

Attn: A. F. Shoemaker

W. Baldwin

Custom Engineering, Inc.

$2805 \mathrm{~S}$ : Tejon St.

Englewood, CO 80110

Attn: C. A. demoraes

DSET

Black Canyon Stage

P.O. Box 185

Phoenix, AZ 85029

Attn:: G. A. Zerlaut

De 1 Manufacturing Co.

905. Monterey Pass Road

Monterey Park, CA 91754

Attn: M. M: Delgado

Desert Research Institute Energy

Systems Laboratory

1500 Buchanan Blvd.

Boulder City, NV 89005

Attn: J. O. Bradley
Donnelly Mirrors, Inc.

49 West Third Street

Holland, MI 49423

Attn: J. A. Knister

E-Systems; Inc: ,

Energy Tech. Center

P.O. Box 226118

Dallas; TX 75266

Attn: R: R. Walters

Easton Utilities Commission 219 North Washington St.

Easton, MD 21601

Attn: Mr.W. H. Corkran, Jr.

Eaton Corporation

Industrial Drives Operations

Cleveland Division

3249 East 80 St.

Cleveland, OH 44104

Attn: R. Glatt

Edison Electric Institute

90 Park Avenue

New York, NY 10016

Attn: L. O. Elsaesser

Electric Power Research Institute (2)

3412 Hillview Avenue

Palo Alto, CA 94303

Attn: Dr. J. Cummings

J. E. Bigger

Energetics

833 E. Nrapahoc strcet

Suite 202

Richardson, TX 75081

Attn: G. Bond

Energy Institute

1700 Las Lomas NE

Albuquerque, NM 87131

Eurodrive, Inc. 2001 W. Main St.

Troy, OH 45373

Attn: S. D. Warner 
DISTŔRBTION (Continued).

Exxon Enterprises (3)

P.O. Box 592

Florham Park, NJ 07923

Attn: J. Hamilton

$$
\begin{aligned}
& \text { P. Joy } \\
& \text { Dr. M. C. Noliand }
\end{aligned}
$$

Florida Solar Energy Center (2)

300 State Road, Suite 401

Cape Canaveral, FL 32920

Attn: C. Beech

D. Block

Ford Aerospace and Communications 3939 Fabian Way

Palo Alto, CA. 94303

Attn: H. J. Sund

Ford Glass Division

Glass Technical Center

25500 West Outer Drive

Lincoln Park, MI 48146

Attn: H. A. Hill

General Atomic

P.O. Box 81608

San Diego, CA 92138

Attn: A. Schwartz

General Electric Co. (2)

P.O. Box 8661

Philadelphia, PA 19101

Attn: W. Pijawka

$$
\text { C. Billingsley }
$$

General Motors

Harrison Radiator Division

Bldg. 6, Dept. 003

Lockport, NY 14094

Attn: L. Brock

General Motors Corporation

Technical Center

Warren, MI 48090

Attn: J. F. Britt

Georgia Institute of Technology

Atlanta; GA 3033 ?

Attn: J. D. Walton
Georgia Power Company

270 Peachtree

P.O. Box 4545

Atlanta, GA 30302

Attn: J. Roberts

Glitsch, Inc.

P.O. Box 226227

Dallas, TX 75266

Attn: R. W. McClain

Haveg Industries, Inc.

1287 E. Imperial Highway

Santa Fe Springs, CA 90670

Attn: J. Flynt

Hexcel

11711 Dublin Blvd.

Dublin, CA 94566

Attn: R. Johnston

Highland Plating

1128 N. Highland

Los Angeles, CA 90038

Attn: M. Faeth

Honeywe 11, Inc.

Energy Resources Center

2600 Ridgeway Parkway

Minneapolis, MN 55413

Attn: J. R. Williams

Insights West

900 wilshire Blvä.

Los Angeles, CA 90017

Attn: J. H. Williams

Jacobs Engineering Co. (2)

251 South Lake Avenue

Pasadena, CA 91101

Attin:: B. Eldridge

R. Morton

Jet Propulsion Laboratory (3)

4800 Oak Grove Drive

Pasadena; CA 91103

Attn: J. Becker

J. Lucas

V. C. Truscello 
DISTRIBUTTION (Continued)

Kingston Industries Corporation 205 Lexing ton Ave.

New York, NY 10016

Lawrence Livermore Laboratory University of California

P.O. Box 808

Livermore, CA 94500

Attn: W. C. Dickinson

Los Alamos National Lab. (3)

Los Alamos, NM 87545

Attn: J. D. Balcomb

C. D. Bankston

D. P. Grimmer

McDonneli Douglas

Astronautics Company (3)

530.1 Bolsa Avenue

Huntington Beach, CA 92647

Attn: J. B. Bl ackmon

J. Rogan

D. Steinmeyer

Morse Chain

Division of Borg-Warner Corp.

4650 steele St.

Denver, Co 80211

Attn: G. Fukayama

Motorola Inc.

Government Electronics Division

8201 E. MCDowell Road

P.O. Box 1417

Scottsdale, AZ 85252

Attn: R. Kendall

New Mexico State University

Solar Energy Department

Las Cruces, NM 88001

Oak Ridge National Laboratory (3)

P.O. Box $Y$

Oale Ridge, TN 37830

Attn: S. I. Kaplan

G. Lawson

W. R. Mixon

Office of Technology Assessment U.S. Congress

Washington, DC 20510

Attn:' R. Rowberg
Omnium G

1815 Orangethorpe Park

Anahe im, CA.92801

Attn: S. P. Lazzara

Owens-Illinois

$1020 \mathrm{~N}$. Westwood

Toledo, $\mathrm{OH} \quad 43614$

Attn: Y.. K. Pei

PPG Industries, Inc.

One Gateway Center

Pittsburgh, PA 15222

Attn: C. R. Frownfelter

PRC Energy Analysis Company 7600 Old Springhouse Road

McLean, VA 22102

Attn: J. Meglan

Parsons of California

3437 S. Airport Way

Stockton, CA 95206

Attn: D. R. Biddle

Progress Industries, Inc.

7290 Murdy Circle

Huntington Beach, CA 92647

Attn: K. Busche

Ronel Technetics, Inc.

501 West Sheridan Rd.

McHenry, IL 60050

Attn: N. Wensel

Schott America

1.1 East 26 th st.

New York, NY 10010

Attn: J. Schrauth

Scientific Applications, Inc. 100 Mercantile, Commerce Bldg. Dallas, TX 75201

Attn: $D r, j, W$, Doane

Scientific Atlanta, Inc. 3845 Pleasantdale Road Atlanta, GA 30340

Attn: A. Ferguson 
DISTRIBUTION (Continued)

Solar Energy Information Center 1536 Cole Blvd.

Golden, CO 80401

Attn: R. Ortiz

Solar Energy Research

Institute (113)

1536 Cole Blva.

Golden; CO 80401

Attn: B. L. Butler

L. G. Dunham (4)

B. P. Gupta

F. Kreith

J. Thornton

K. Touryan

N. Woodley

D. W. Kearney

C. Bishop

B. Feasby

A. Lewandowski (100)

Solar Energy Technology

Rocketdyne Division

6633 Canoga Avenue

Canoga Park, CA 91304

Attn: J. M. Friefeld

Solar Kinetics.Inc.

P.O. Box 47045

8120 Chancellor Row

Dallas, TX 75247

Attn: G. Hutchinson

Southwest Research Institute P. U. Box $28 b \perp 0$

San Antonio, TX 78284

Attn: D. M. Deffenbaugh

Stanford Research Institute

Menlo Park, CA 94025

Attn: A. J. Slemmons

Stearns-Roger

4500 Cherry Creek

Denver, CO 80217

Attn: W. R. Lang

W. B. Stine

317 Monterey Rd., Apt. 22

South Pasadena, CA 91303
Sun Gas Company

Suite 800,2 No. Pk. E

Dallas, TX 75231

Attn: R.C. Clark

Sun-Heet, Inc.

2624 So. Zunl

Englewood, CO 80110

Sundstrand Electric Power

4747 Harrison Avenue

Rockford, IL 61101

Attn: A. W. Ad am

Sunpower Systems Corp.

510 S. 52nd St., Suite 101

Tempe, AZ 85281

Attn: W. C. Matlock

Suntec Systems, Inc.

2101 Woodale Drive

St. Paul, MN 55110

Attn: L. W. Rees

Swedlow, Inc.

12122 Western Avenue

Garden Grove, CA 92645

Attn: E. Nixon

3M-Decorative Products Division 209-2N 3M Center

St. Paul, MN . 55101

Attn: B. Benson

3M-Product Development

Energy Control Products

207-1W. 3M Center

St. Paul, MN 55101

Attn: J. R. Roche

Texas Tech University

Dept. of Electrical Engineering

P.O. Box 4709

Lubbock, TX 79409

Attn: J. D. Reichert

TRW, Inc.

Energy Systems Group of TRW, Inc.

One Space Park Bldg. R4, Room 2074

Redondo Beach; CA 90278

Attn: J. M. Cherne 
Toltec Industries, Inc. 40 th and East Main

Clear Lake, IA 50428

Attn: D. Chenault

U.S. Department of Energy (3)

Albuquerque Operations office P.O. Box 5400

Albuque rque, NM 87185

Attn: G. N. Pappas

C. B. Quinn

J. Weisiger

U.S. Department of Energy

Division of Energy Storage Systems

Washington, DC 20545

Attn: J. Gahimer

U.S. Department of Energy (8)

Division of Solar Thermal Energy Systems

Washington, DC 20585

Attn: W. W. Auer

G. W. Braun

J. E. Greyerbiehl

M. U. Gutstein

L. Melamed

J. E. Rannels

F: Wilkins

J. Dollard

U.S. Department of Energy (.2)

San Francisco Operations office 1333 Broadway, Wells Fargo Bldg. Oakland, CA 94612

Attn: R. W. Hughey

University of Kansas Center for Research, CRINC

2291 Irving Hall Rd.

Lawrence, KS 66045

Attn: R. F. Riordan

University of New Mexico (2) Department of Merhanical Eng. Albuquerque, NM 87113

Attn: M. W. Wilden

W. A. Cross
Viking Solar systems

3223 North Verdugo Road

Glendale, CA 91208

Attn: G. Goranson

Winsmith

Div. of UMC Industries, Inc. Springville, NY 14141

Attn: R. Bhise

Wyle Laboratories

7800 Governor's Drive West

Huntsville, AL 35807

Attn: R. Losey

1520 T. J. Hoban

1530 W. E. Caldes

1550 F. W. Neilson

2320 K. L. Gillespie

2323 C. M. Gabriel

2324 R. S. Pinkham

2326 G. M. Heck

3161 J. E. Mitchell

3600 R. W. Hunnicutt

Attn: H. H. Pastorius, 3640

3700 J. C. Strassell

4000 A. Narath

4231 J. H. Renken

4700 J. H.'Scott

4710 G. E. Brandvold

4713 B. W. Marshall

4714 R. P. Stromberg (20)

4715 R. H. Braasch

4718 E. Burgess

4719 D. G. Schueler

4720 V. L. Dugan (100)

4721 Author (20)

4721 J. V. Otts

4722 J. F: Banas

4723 W. P. Schimmel

4725 J. A. Leonard

4730 H. M. Stoller

5510 D. B. Hayes

5513 D. W. Larson

5520 T. B. Lane

5523 R., C. Reuter

5810. R. G. Kepler

5820 R. E. Whan

5830 M. J. Davis

5833. J. L. Jellison

5840 N. Magnani 
DISTRIBUTION (Continued)

$\begin{array}{ll}8266 & \text { E. A. Aas (2) } \\ 8450 & \text { R. C. Wayne } \\ 8451 & \text { C. F. Melius } \\ 8452 & \text { A. C. Skinrood } \\ 8452 & \text { T. Bramlette } \\ 8453 & \text { W. G. Wilson } \\ 3141 & \text { T. L. Werner (5) } \\ 3151 . & \text { W. L. Garner (3) } \\ & \text { (Unlimited Release) } \\ & \text { For DOE/TIC } \\ & \text { (Unlimited Release) } \\ 6011 & \text { Patents }\end{array}$


Rec'd by *

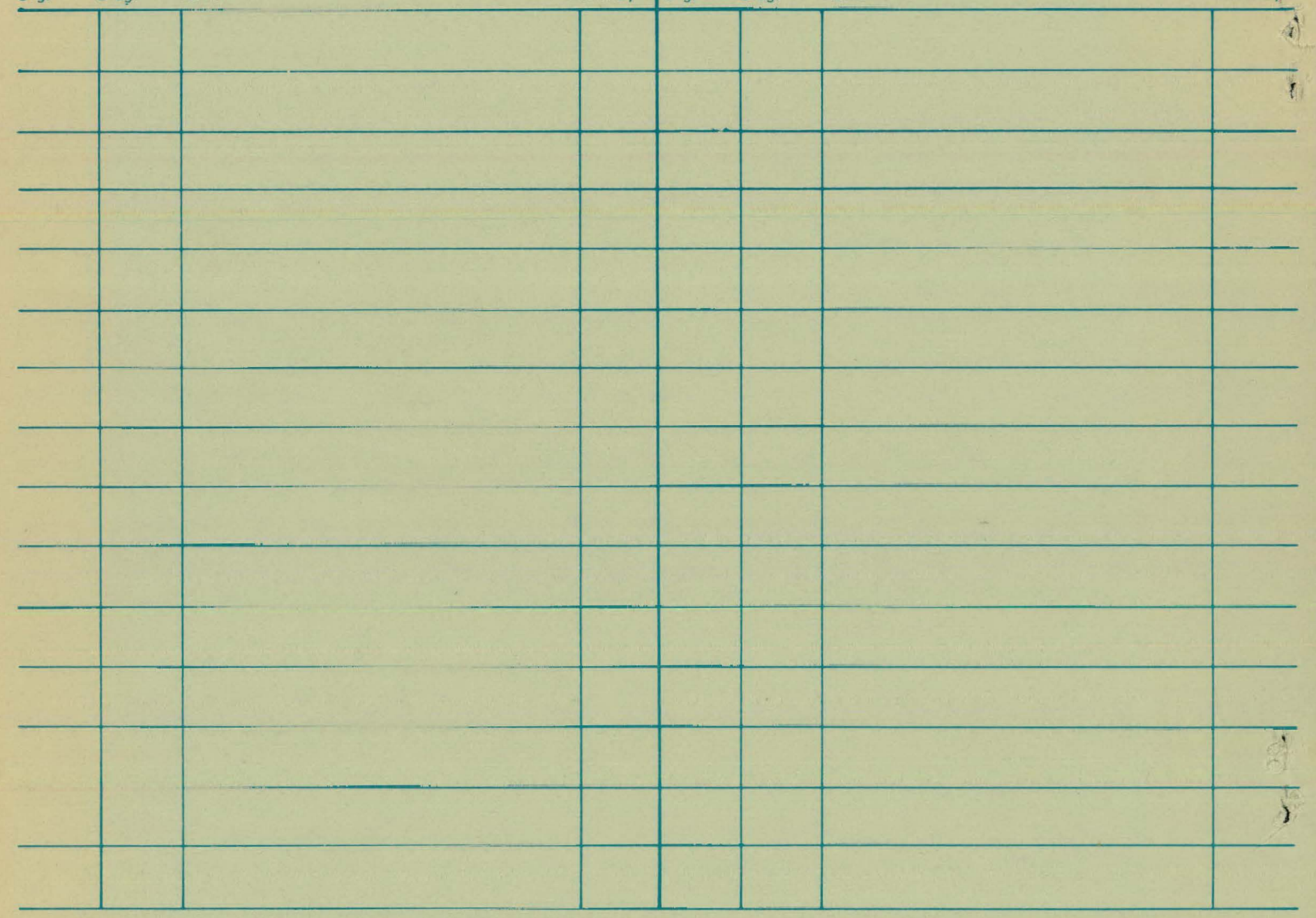

